\title{
Electron and Photon Scattering on Three-Nucleon Bound States
}

\author{
J. Golak, R. Skibiński, H. Witała \\ M. Smoluchowski Institute of Physics, \\ Jagiellonian University, PL-30059 Kraków, Poland \\ W. Glöckle \\ Institut für Theoretische Physik II, \\ Ruhr Universität Bochum, D-44780 Bochum, Germany \\ A. Nogga \\ Forschungszentrum Jülich, IKP (Theorie), D-52425 Jülich, Germany \\ H. Kamada \\ Department of Physics, Faculty of Engineering, \\ Kyushu Institute of Technology, 1-1 Sensuicho, \\ Tobata, Kitakyushu 804-8550, Japan
}




\begin{abstract}
A big spectrum of processes induced by real and virtual photons on the ${ }^{3} \mathrm{He}$ and ${ }^{3} \mathrm{H}$ nuclei is theoretically investigated through many examples based on nonrelativistic Faddeev calculations for bound and continuum states. The modern nucleon-nucleon potential AV18 together with the threenucleon force UrbanaIX is used. The single nucleon current is augmented by explicit $\pi$ - and $\rho$-like two-body currents which fulfill the current continuity equation together with the corresponding parts of the AV18 potential. We also employ the Siegert theorem, which induces many-body contributions to the current operator. The interplay of these different dynamical ingredients in the various electromagnetic processes is studied and the theory is compared to the experimental data. Overall we find fair to good agreement but also cases of strong disagreement between theory and experiment, which calls for improved dynamics. In several cases we refer the reader to the work of other groups and compare their results with ours. In addition we list a number of predictions for observables in different processes which would challenge this dynamical scenario even more stringently and systematically.
\end{abstract}

PACS numbers: $21.45+\mathrm{v}, 21.10-\mathrm{k}, 25.10+\mathrm{s}, 25.20-\mathrm{x}$ 


\section{Contents}

$\begin{array}{ll}\text { I. Introduction } & 5\end{array}$

II. Formalism in the Faddeev scheme $\quad 9$

$\begin{array}{lr}\text { III. Current operators } & 19\end{array}$

A. The single nucleon current 20

B. The Siegert approach 22

C. $\pi$ and $\rho$-like meson exchange currents 25

IV. The Observables $\quad 32$

V. The Performance $\quad 45$

VI. Comparison with data $\quad 55$

A. Elastic electron scattering on ${ }^{\mathbf{3}} \mathrm{He}$ and ${ }^{\mathbf{3}} \mathrm{H}$

B. Inclusive electron scattering on ${ }^{\mathbf{3}} \mathrm{He}$ and ${ }^{\mathbf{3}} \mathrm{H}$

C. Electron induced pd breakup of ${ }^{3} \mathrm{He} \quad 78$

D. $\mathrm{Nd}$ radiative capture and the time reversed $\mathrm{Nd}$ photodisintegration of $3 \mathrm{~N}$ bound $\begin{array}{ll}\text { states. } & 87\end{array}$

E. Three-nucleon photodisintegration of the ${ }^{3} \mathrm{He} \quad 97$

$\begin{array}{ll}\text { VII. Predictions } & 101\end{array}$

A. Inclusive electron scattering on ${ }^{\mathbf{3}} \mathrm{He} \quad 101$

B. Electron induced pd breakup of ${ }^{\mathbf{3}} \mathrm{He} \quad 106$

C. Semiexclusive nucleon knockout processes 111

D. The electron induced complete 3N breakup process 118

E. Spin dependent momentum distributions of polarized proton-deuteron clusters in polarized ${ }^{\mathbf{3}} \mathrm{He} \quad 123$

F. 3N Photodisintegration of ${ }^{\mathbf{3}} \mathrm{He} \quad 128$

$\begin{array}{lr}\text { VIII. Addendum } & 135\end{array}$

IX. Summary and Outlook 138 
Acknowledgments

References 


\section{INTRODUCTION}

Real and virtual photon induced processes in the three-nucleon $(3 \mathrm{~N})$ system have been studied for a long time and these investigations go on with intensity. The reason is that beyond the deuteron the $3 \mathrm{~N}$ system in the form of ${ }^{3} \mathrm{He}$ has been always considered since the very beginning of nuclear physics $[1,2]$ as a challenge to be understood in terms of the available state of the art forces. Then the next question followed naturally: what is the response of the $3 \mathrm{~N}$ bound state to real and virtual photon absorption ? Again answers have been searched for over the many years to the best of the available physical insights and technical feasibilities. Here we point just to a few early studies [3, 4] and refer the reader to the various reviews given below for the long history of that research.

While the $3 \mathrm{~N}$ bound states were numerically mastered already in the seventies and early eighties using nucleon-nucleon (NN) forces with realistic and complex spin-momentum structures [5-10] and later on adding first models for $3 \mathrm{~N}$ forces $[10-15]$, the technical challenges for the $3 \mathrm{~N}$ continuum with the complex asymptotic boundary conditions were much more demanding. But in the last 10-15 years also the $3 \mathrm{~N}$ continuum got more and more under control [16-21], which opened solid theoretical access to the great diversity of inelastic real and virtual photon induced reactions on ${ }^{3} \mathrm{He}$ and the nucleon-deuteron $(\mathrm{Nd})$ capture processes. There has been, and this is going on, an intensive interplay and reciprocal stimulation of theory and experiment, which justifies, as we think, a review of the present state of the art.

Elastic electron scattering on ${ }^{3} \mathrm{He}\left({ }^{3} \mathrm{H}\right)$ has been reviewed many times over the years [2226]. The inclusive process ${ }^{3} \mathrm{He}\left(e, e^{\prime}\right)$ has been reviewed in [26]. A very informative monograph on electron induced processes on nuclei including the $3 \mathrm{~N}$ system is [27]. Semiexclusive and above all exclusive electron induced processes on ${ }^{3} \mathrm{He}$ came into the focus only with the highduty cycle electron accelerators (NIKHEF, MAMI, Jlab) and reviews about those processes in the $3 \mathrm{~N}$ system are not known to us. A good collection of references to old calculations on the photodisintegration of ${ }^{3} \mathrm{He}$ can be found in [28]. Recent work on these processes is discussed and cited in [26].

Variational approaches and rudimentary treatments of the $3 \mathrm{~N}$ continuum in electromagnetically induced processes were used before the sixties and still in the early seventies and we refer the reader to the literature quoted in the above listed reviews. Then with the Faddeev formulation of the three-body system [29] or the equivalent Alt-Grassberger-Sandhas 
(AGS) equations [30], where the latter ones are ideally suited for finite rank forces, a new epoch started. In the following we shall not distinguish between the two and just call them for short "Faddeev approach". At that time, due to the lack of sufficiently strong computational resources, the nuclear potentials were chosen in a quite simple form, low rank separable ones. Very first calculations for electrodisintegration of ${ }^{3} \mathrm{He}$ and ${ }^{3} \mathrm{H}$ in the Faddeev scheme were performed in [31], where the $3 \mathrm{~N}$ bound state was treated correctly but in the final $3 \mathrm{~N}$ continuum state only the interaction within the spectator pair was kept (the two nucleons which have not absorbed the photon under a single nucleon current assumption).

Very similar in nature and techniques is the photodisintegration, where the first Faddeev calculation for the $3 \mathrm{~N}$ continuum appeared in [32] and where the importance of the rescattering with the spectator nucleons was emphasized. One step further was the work in [33] where for the two- and three-body photodisintegration of ${ }^{3} \mathrm{He}\left({ }^{3} \mathrm{H}\right)$ both, ground state and 3N continuum, were treated consistently as solutions of the Schrödinger equation with the same 3N Hamiltonian. This exact treatment, though still with simple NN forces, already allowed one to ask detailed questions [34] like the suppression of the isospin $\mathrm{T}=1 / 2$ contribution in three-body photodisintegration of ${ }^{3} \mathrm{He}$. Then the first calculation for two-body electrodisintegration of ${ }^{3} \mathrm{He}\left({ }^{3} \mathrm{H}\right)$ came up in [35]. Though also the formalism for three-body disintegration in the context of separable forces was formulated, limitations of computer resources prevented their realization. It then took quite some time that the three-body electrodisintegration has been treated [36] using simple $s$-wave local forces in an unitary pole expansion or only in the form of the unitary pole approximation. The conclusion was again that a proper description has to take into account contributions from the complete multiple scattering series, or in other words, that final state interaction (FSI) are important. Due to the lack of kinematically complete breakup data, the calculation of [36] was applied to a set of existing inclusive data, where the two- and three-body electrodisintegration processes are both involved.

Physically and formally closely related to electron induced processes is the protondeuteron $(\mathrm{pd})$ radiative capture reaction, where a first configuration space $3 \mathrm{~N}$ calculation based on solutions of the Faddeev equation for the $3 \mathrm{~N}$ bound state and $3 \mathrm{~N}$ scattering states appeared in [37] using the Reid NN force [38]. Thereby, as in the following studies [39-42], the interest was in the sensitivity of tensor analyzing powers to properties of the $3 \mathrm{~N}$ bound state and to the NN tensor forces. The treatment of the initial state interaction in the pd 
capture processes turned out to be very crucial as well as the inclusion of higher NN force components. In [39] realistic $\mathrm{NN}$ forces and even $3 \mathrm{~N}$ forces were used in a consistent $3 \mathrm{~N}$ Faddeev treatment for both the ground state and the continuum states. In [40] separable forces were employed but also an Ernst-Shakin-Thaler-expansion form of the Paris potential.

At very low energies (neutron-deuteron) nd capture was treated in [43] using a configuration space Faddeev method and realistic NN and 3N forces. The method of correlated orthogonal states [44] represents the continuum to some extent and puts in short-range correlations. Although the states are not proper solutions of the $3 \mathrm{~N}$ Hamiltonian, their use in studying inclusive response functions clearly showed significant improvements over plane-wave impulse approximation results and underlined the importance of treating the correlations between the three nucleons in the final state as consequently as in the $3 \mathrm{~N}$ bound state.

Another development was the Euclidean response method [45] applied to inclusive responses. By path integral techniques one calculates the Laplace transform of the response functions and compares them to the corresponding Laplace transformed data. This is an exact method and includes the full dynamics of the chosen Hamiltonian. Related to that are approaches with Stieltjes transforms [46] or transformations by a Lorentz kernel [47].

Around that time the first calculations appeared, where realistic NN forces, with all their complexities and including all the relevant higher NN force components, were applied to the $\operatorname{pd}(\mathrm{nd})$ and three-nucleon electrodisintegration of ${ }^{3} \mathrm{He}\left({ }^{3} \mathrm{H}\right)$ in the Faddeev scheme [48, 49]. In that formulation the pd and ppn breakup of ${ }^{3} \mathrm{He}$ induced by an external probe can be calculated in "one shot" solving a Faddeev like integral equation and avoiding the nasty low order rescattering processes occurring in the separate treatment of the $3 \mathrm{~N}$ continuum [36]. For inclusive scattering a convenient short cut was found in $[50,51]$ using the closure relation for the eigenstates of the Hamiltonian. In this manner, one avoids the explicit numerical integrations over all the available two-body and three-nucleon disintegration configurations.

In the older investigations mostly only the nonrelativistic single nucleon current operator has been used. For real photon induced processes it was supplemented by the Siegert approach, which takes some exchange currents into account. This is insufficient and the explicit use of two-body currents (and possibly three-body contributions when a three nucleon force $(3 \mathrm{NF})$ is included) is required. These dynamical ingredients are as complicated as nuclear forces and therefore progress is slow. An important practical step was performed in [52, 53] 
by associating two-body currents to NN forces through the continuity equation. In the case of the AV18 NN force [54] that recipe has been used quite often [26] and is still applied. Closely related studies connecting NN forces and two-body currents appeared in $[55,56]$.

Nearly all of the results shown in this review are based on our own work using the Faddeev scheme in a purely nucleonic Hilbert space. There are also other groups, which investigate real and virtual photon induced processes on light systems. For the wealth of insight and achievements in the case of the deuteron we refer to $[57,58]$. Here we focus just on the $3 \mathrm{~N}$ system. The group in Pisa uses hyperspherical harmonic expansions of different types and treats bound and continuum states consistently. They use modern nuclear forces in all their complexities together with related currents. Their focus is mostly on processes at very low energies [59]. This includes pd radiative capture, inclusive threshold electron scattering on ${ }^{3} \mathrm{He}$, and pd breakup electrodisintegration of ${ }^{3} \mathrm{He}$. The Urbana-Argonne group relies beside variational approaches on the Green-function-Monte-Carlo method [60]. A good overview on the theory and their important results can be found in [26]. In the $3 \mathrm{~N}$ system this comprises work on the elastic form factors, short-range correlations related to the Coulomb sum rule, Nd capture reactions, and Euclidean inclusive response functions. The group in Trento uses the Lorentz integral transform (LIT) method [47] and employs also hyperspherical harmonic expansions. In this method one avoids the direct treatment of the continuum which requires the handling of the complex boundary conditions. Instead that method converts the continuum problem into a bound state problem. The price to be paid is an inversion of auxiliary Lorentz transformed amplitudes. The mathematical properties of that technique are displayed in [61]. This method is being applied not only to the $3 \mathrm{~N}$ system but is powerful enough to go beyond $A=3$ using ideas of effective force expansions [62-64]. More recently the Hanover group also started to thoroughly investigate the $3 \mathrm{~N}$ continuum and photon induced reactions therein [65-67]. The new feature is the explicit inclusion of the $\Delta$-degree of freedom. Thus the Hilbert space is the direct sum of NNN and NN $\Delta$ states. In this manner a certain subset of $3 \mathrm{~N}$ forces is taken care of as well as consistent two-body currents.

Last but not least we would like to point to the very rich list of investigations by J.M. Laget who uses a diagrammatic approach. That work has stimulated many experimental investigations and sheds light on the reaction mechanisms. A recent paper [68] discusses electrodisintegration of few-body systems high in momenta above our nonrelativistic domain 
but also provides many references to earlier studies, which are relevant to the work discussed in this review.

For all technical details used by these other groups we refer the reader to the cited literature. We shall provide, however, information on their results at the appropriate places in section VI.

This review is organized as follows. In Section II we describe our approach in the Faddeev scheme for the great diversity of photon induced processes. A brief review on electromagnetic currents is given in Section III. The observables are defined in Section IV. Then Section V describes the way we technically perform the calculations. Section VI is devoted to a comparison of our theoretical results and some selected results by other groups to the data. Much remains, however, to be done and we present in Section VII an incomplete and subjective list of theoretical predictions, some of which will hopefully be testified in experiments in the near future. In Section VIII we provide remarks on several issues relevant in the $3 \mathrm{~N}$ system which have not been addressed directly in this review. We end up with a summary and outlook in Section IX.

\section{FORMALISM IN THE FADDEEV SCHEME}

Let us start with a heuristic approach toward the photon induced complete breakup of ${ }^{3} \mathrm{He}$. Once the photon has been absorbed inside ${ }^{3} \mathrm{He}$, the three nucleons are released but on the way of leaving the space spanned by the ${ }^{3} \mathrm{He}$ state they interact strongly. This is illustrated in Fig. 1. Clearly this infinite set of diagrams summarizes all what can happen in the $3 \mathrm{~N}$ breakup process under the condition that the three nucleons are interacting by pairwise forces. Because of the strength of nuclear forces that series is generally diverging for c.m. energies in the $3 \mathrm{~N}$ system below the pion production threshold. It has to be summed up to infinite order. We follow here the Faddeev scheme and perform first a partial resummation of the $\mathrm{NN}$ forces into NN t-operators. Apparently aside from the very first term without any interaction after the photon absorption process $\left(U_{0}^{(0)}\right)$ that set of diagrams can be split into 3 subsets according to the utmost left pair force

$$
U_{0}=U_{0}^{(0)}+U_{0}^{(1)}+U_{0}^{(2)}+U_{0}^{(3)}
$$

where $U_{0}^{(i)}$ stands for the subset with $V_{j k}$ to the left $(j \neq i \neq k)$. 


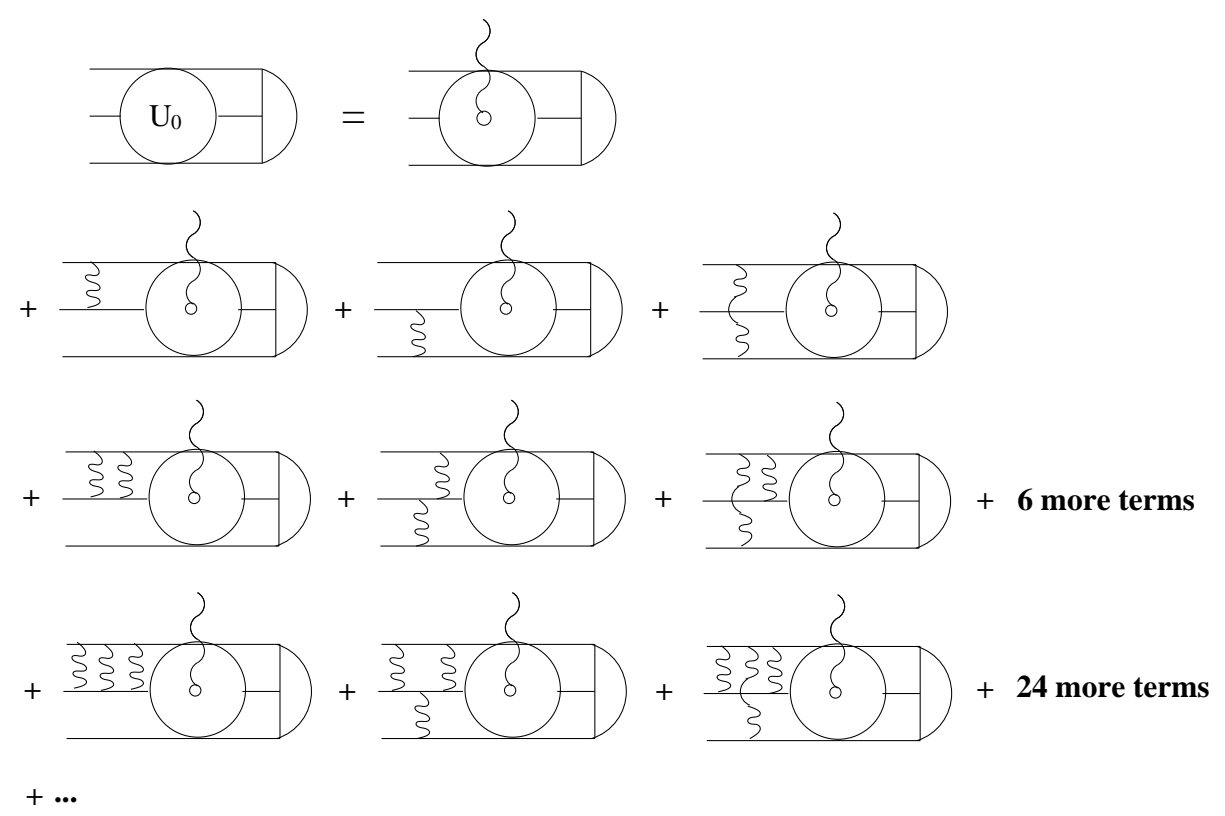

FIG. 1: The multiple scattering series for a $3 \mathrm{~N}$ breakup amplitude due to photon absorption. The half moon to the very right stands for the ${ }^{3} \mathrm{He}$ state, the circle with the wiggly line attached to it for the one-photon absorption process and the wiggly lines for NN forces acting between all pairs to first order, second order etc. For the sake of notation simplicity the action of $3 \mathrm{~N}$ forces has been dropped. The three horizontal lines between the action of NN forces and between the photon absorption and the NN forces stand for a free $3 \mathrm{~N}$ propagation and the three final horizontal lines to the very left represent the three final nucleons (their momentum eigenstates). 
Let us regard the first few terms for $U_{0}^{(1)}$ in Fig. 2:

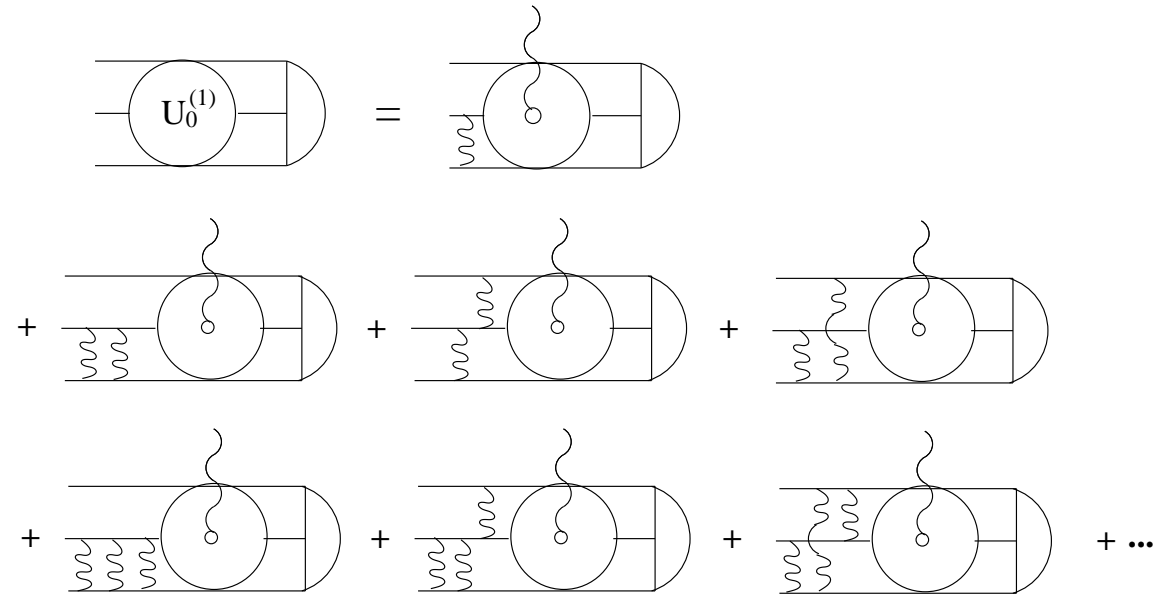

FIG. 2: The subset of diagrams ending with $V_{23}$ to the very left. Symbols as in Fig. 1.

By the very definition of the three subsets this equals

$$
U_{0}^{(1)}=V_{23} G_{0} \mathcal{O}|\Psi\rangle+V_{23} G_{0}\left(U_{0}^{(1)}+U_{0}^{(2)}+U_{0}^{(3)}\right)
$$

where $\mathcal{O}$ is the photon absorption operator, $|\Psi\rangle$ the ${ }^{3}$ He state and $G_{0}$ the free $3 \mathrm{~N}$ propagator. We combine the terms with $U_{0}^{(1)}$ on the left hand side

$$
\left(1-V_{23} G_{0}\right) U_{0}^{(1)}=V_{23} G_{0} \mathcal{O}|\Psi\rangle+V_{23} G_{0}\left(U_{0}^{(2)}+U_{0}^{(3)}\right)
$$

invert

$$
U_{0}^{(1)}=\left(1-V_{23} G_{0}\right)^{-1} V_{23} G_{0} \mathcal{O}|\Psi\rangle+\left(1-V_{23} G_{0}\right)^{-1} V_{23} G_{0}\left(U_{0}^{(2)}+U_{0}^{(3)}\right)
$$

and introduce the NN t-operator $t_{23}$

$$
t_{23} \equiv\left(1-V_{23} G_{0}\right)^{-1} V_{23}
$$

Obviously, $t_{23}$ obeys the two-body Lippmann-Schwinger equation

$$
t_{23}=V_{23}+V_{23} G_{0} t_{23}
$$

This leads to

$$
U_{0}^{(1)}=t_{23} G_{0} \mathcal{O}|\Psi\rangle+t_{23} G_{0}\left(U_{0}^{(2)}+U_{0}^{(3)}\right) .
$$

Two more equations for $U_{0}^{(2)}$ and $U_{0}^{(3)}$ arise in exactly the same manner. 
Now we make use of the identity of the three nucleons. Since the photon absorption operator $\mathcal{O}$ has to be symmetrical under exchange of the three nucleons and the ${ }^{3}$ He state is antisymmetrical one immediately obtains

$$
U_{0}^{(2)}=P_{12} P_{23} U_{0}^{(1)}
$$

and

$$
U_{0}^{(3)}=P_{13} P_{23} U_{0}^{(1)}
$$

where $P_{i j}$ interchanges nucleons $i$ and $j$. It is convenient to define [69]

$$
P \equiv P_{12} P_{23}+P_{13} P_{23}
$$

and we obtain

$$
U_{0}^{(1)}=t_{23} G_{0} \mathcal{O}|\Psi\rangle+t_{23} G_{0} P U_{0}^{(1)}
$$

This is already a Faddeev type integral equation, which after iteration leads to the multiple scattering series, now formulated in terms of $\mathrm{NN}$ t-operators

$$
U_{0}^{(1)}=t G_{0} \mathcal{O}|\Psi\rangle+t G_{0} P t G_{0} \mathcal{O}|\Psi\rangle+t G_{0} P t G_{0} P t G_{0} \mathcal{O}|\Psi\rangle+\ldots
$$

This is graphically depicted in Fig. 3. The whole breakup amplitude is then given as

$$
U_{0}=U_{0}^{(0)}+(1+P) U_{0}^{(1)}
$$

Here $U_{0}^{(0)}$ is obtained by a simple quadrature and $U_{0}^{(1)}$ arises as solution of the one Faddeevlike equation (11).

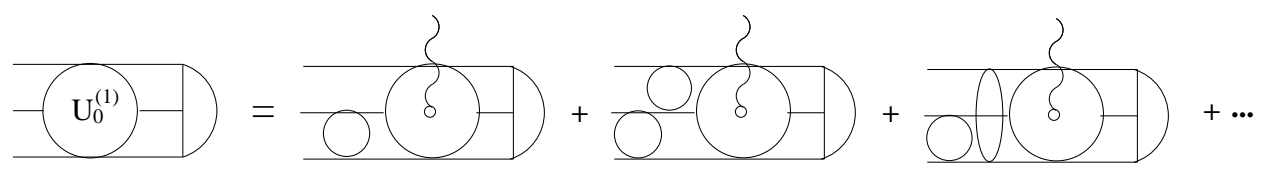

FIG. 3: In comparison to Fig. 2 the NN forces are now replaced by NN $t$-operators represented as circles or as an oval in the case of the pair 13. In the second and higher orders clearly only consecutive circles acting on different pairs can appear.

Written in a more definite manner as matrix element the breakup amplitude reads

$$
U_{0}=\left\langle\phi_{0}|\mathcal{O}| \Psi\right\rangle+\left\langle\phi_{0}|(1+P)| U\right\rangle
$$


where the amplitude $|U\rangle$ obeys according to (11)

$$
|U\rangle=t G_{0} \mathcal{O}|\Psi\rangle+t G_{0} P|U\rangle
$$

We dropped the index 23 on t since one can choose any pair and we introduced the free $3 \mathrm{~N}$ state $\left\langle\phi_{0}\right|$. Since $\mathcal{O}|\Psi\rangle$ and $(1+P)|U\rangle$ are totally antisymmetrical, we can assume $\left\langle\phi_{0}\right|$ to be antisymmetrical as well.

Let us now re-derive that result in a more standard algebraic manner including also $3 \mathrm{~N}$ forces. The general form of the nuclear matrix element for an electroweak probe represented by a symmetric operator $\mathcal{O}$ is given as

$$
N=\left\langle\Psi_{f}^{(-)}|\mathcal{O}| \Psi_{i}\right\rangle
$$

Here $\left|\Psi_{i}\right\rangle$ is the initial nucleus state and $\left\langle\Psi_{f}^{(-)}\right|$the final scattering state with asymptotic quantum numbers $f$. It is generated as [70]

$$
\left|\Psi_{f}^{(-)}\right\rangle=\lim _{\epsilon \rightarrow 0^{+}} \frac{-i \epsilon}{E-i \epsilon-H}\left|\phi_{f}\right\rangle .
$$

In the three-nucleon system and for inelastic processes $f$ stands either for asymptotic $\mathrm{Nd}$ or $3 \mathrm{~N}$ quantum numbers. In the latter case we already introduced the fully antisymmetrical state $\left|\phi_{0}\right\rangle$, which in our notation is given as

$$
\left|\phi_{0}\right\rangle=(1+P)\left|\varphi_{0}\right\rangle
$$

where $\left|\varphi_{0}\right\rangle$ in the nonrelativistic regime is conveniently expressed in terms of Jacobi momenta

$$
\left|\varphi_{0}\right\rangle \equiv\left(1-P_{23}\right)|\vec{p} \vec{q}\rangle \equiv|\vec{p}\rangle_{a}|\vec{q}\rangle
$$

Depending on which pair of nucleons is singled out there are three choices for the Jacobi momenta. Let us choose one of them and define

$$
\begin{gathered}
\vec{p}=\frac{1}{2}\left(\vec{k}_{2}-\vec{k}_{3}\right) \\
\vec{q}=\frac{2}{3}\left[\vec{k}_{1}-\frac{1}{2}\left(\vec{k}_{2}+\vec{k}_{3}\right)\right],
\end{gathered}
$$

where the $\vec{k}_{i}$ are the individual laboratory momenta. In the notation (19) we dropped additional spin and isospin quantum numbers.

Let us now firstly stick to the $3 \mathrm{~N}$ breakup channel, thus $\left|\phi_{f}\right\rangle=\left|\phi_{0}\right\rangle$. 
The Hamiltonian $H$ occurring in (17) contains $\mathrm{NN}$ and $3 \mathrm{~N}$ forces on top of the kinetic energy $H_{0}$

$$
H=H_{0}+\sum_{i<j} V_{i j}+V_{123}
$$

One way to handle the $3 \mathrm{~N}$ force operator $V_{123}$ is to split it into 3 parts

$$
V_{123}=V^{(1)}+V^{(2)}+V^{(3)}
$$

where $V^{(i)}$ is symmetrical under exchange of nucleons $j$ and $k$. Such a splitting is always possible. Thus it appears natural to combine the interactions as

$$
H=H_{0}+\left(V_{12}+V^{(3)}\right)+\left(V_{23}+V^{(1)}\right)+\left(V_{31}+V^{(2)}\right) \equiv H_{0}+\sum_{i=1}^{3}\left(V_{i}+V^{(i)}\right) .
$$

We introduced the standard and convenient notation $V_{i} \equiv V_{j k},(j \neq i \neq k)$. Clearly both terms $V_{i}$ and $V^{(i)}$ are symmetrical under exchange of nucleons $j$ and $k$.

Now using the well known identity between the full resolvent operator $G^{(-)}$occurring in (17) and the free resolvent operator

$$
G_{0}^{(-)} \equiv \frac{1}{E-i \epsilon-H_{0}}
$$

namely

$$
G^{(-)}=G_{0}^{(-)}+G_{0}^{(-)} \sum_{i=1}^{3}\left(V_{i}+V^{(i)}\right) G^{(-)}
$$

one obtains the Lippmann-Schwinger equation for $\left|\Psi_{0}^{(-)}\right\rangle$as

$$
\left|\Psi_{0}^{(-)}\right\rangle=\left|\phi_{0}\right\rangle+G_{0}^{(-)} \sum_{i=1}^{3}\left(V_{i}+V^{(i)}\right)\left|\Psi_{0}^{(-)}\right\rangle \text {. }
$$

This suggests a decomposition of the total state into three parts and using again the identity of the three nucleons leads to [71]

$$
\left|\Psi_{0}^{(-)}\right\rangle=(1+P)\left|\psi^{(-)}\right\rangle
$$

where $\left|\psi^{(-)}\right\rangle$obeys the Faddeev-like equation

$$
\left|\psi^{(-)}\right\rangle=\left|\varphi_{0}^{(-)}\right\rangle+G_{0}^{(-)} t^{(-)} P\left|\psi^{(-)}\right\rangle+\left(1+G_{0}^{(-)} t^{(-)}\right) G_{0}^{(-)} V^{(1)}(1+P)\left|\psi^{(-)}\right\rangle .
$$

The driving term is

$$
\left|\varphi_{0}^{(-)}\right\rangle=\left(1+G_{0}^{(-)} t^{(-)}\right)\left|\varphi_{0}\right\rangle \equiv|\vec{p}\rangle_{a}^{(-)}|\vec{q}\rangle
$$


Thus in the $2 \mathrm{~N}$ subsystem the antisymmetric free state $|\vec{p}\rangle_{a}$ is replaced by the two-body scattering state $|\vec{p}\rangle_{a}^{(-)}$.

The result for $\left|\Psi_{0}^{(-)}\right\rangle$can now be inserted into the nuclear matrix element (16):

$$
N=\left\langle\psi^{(-)}|(1+P) \mathcal{O}| \Psi_{i}\right\rangle=\left\langle\varphi_{0}\left|\left(1+t G_{0}\right)(1-K)^{-1}(1+P) \mathcal{O}\right| \Psi_{i}\right\rangle,
$$

where

$$
K=P t G_{0}+(1+P) V^{(1)} G_{0}\left(1+t G_{0}\right)
$$

is the adjoint kernel to the one occurring in (29).

The heuristically derived result (14) valid for $V^{(i)}=0$ can now be recovered easily. We use the identity

$$
\left(1+t G_{0}\right)\left(1-P t G_{0}\right)^{-1}=1+(1+P)\left(1-t G_{0} P\right)^{-1} t G_{0}
$$

and obtain

$$
\begin{gathered}
N=\left\langle\varphi_{0}|(1+P) \mathcal{O}| \Psi_{i}\right\rangle+\left\langle\varphi_{0}\left|(1+P)\left(1-t G_{0} P\right)^{-1} t G_{0}(1+P) \mathcal{O}\right| \Psi_{i}\right\rangle \\
=\left\langle\phi_{0}|\mathcal{O}| \Psi_{i}\right\rangle+\left\langle\phi_{0} \mid U^{\prime}\right\rangle
\end{gathered}
$$

with $\left|U^{\prime}\right\rangle$ given by the integral equation

$$
\left|U^{\prime}\right\rangle=t G_{0}(1+P) \mathcal{O}\left|\Psi_{i}\right\rangle+t G_{0} P\left|U^{\prime}\right\rangle
$$

This has to be compared to the result given in (14) and (15). Since $\mathcal{O}\left|\Psi_{i}\right\rangle$ is antisymmetrical, we obtain

$$
t G_{0}(1+P) \mathcal{O}\left|\Psi_{i}\right\rangle=3 t G_{0} \mathcal{O}\left|\Psi_{i}\right\rangle
$$

Consequently, $\left|U^{\prime}\right\rangle=3|U\rangle$ and the second term in (34) yields $\left\langle\phi_{0} \mid U^{\prime}\right\rangle=3\left\langle\phi_{0} \mid U\right\rangle$ which equals the second term in (14). This is obvious by applying $(1+P)$ to the antisymmetrical state $\left\langle\phi_{0}\right|$ on the left yielding again a factor of 3 . This completes the verification of the heuristically derived result.

Including now the $3 \mathrm{NF}$ we define according to the expression (31)

$$
\left|\tilde{U}^{\prime}\right\rangle \equiv(1-K)^{-1}(1+P) \mathcal{O}\left|\Psi_{i}\right\rangle
$$

or the equivalent integral equation

$$
\left|\tilde{U}^{\prime}\right\rangle=(1+P) \mathcal{O}\left|\Psi_{i}\right\rangle+\left(P t G_{0}+(1+P) V^{(1)} G_{0}\left(1+t G_{0}\right)\right)\left|\tilde{U}^{\prime}\right\rangle .
$$


The breakup matrix element is determined by means of $\left|\tilde{U}^{\prime}\right\rangle$ according to (29) - (31)

$$
N={ }^{(-)}\left\langle\varphi_{0} \mid \tilde{U}^{\prime}\right\rangle
$$

Unfortunately, the form (38), although suitable for separable forces, is not appropriate for numerical applications with realistic interactions because of the presence of the permutation operator $P$ to the very left in the first part of the kernel[72]. It would "smear out" the position of the deuteron singularity in the $\mathrm{NN}$ t-operator. To rewrite (38) into a suitable form we use the following obvious identities

$$
\begin{gathered}
1+P=\frac{1}{2} P(1+P), \\
\frac{1}{2} P(P-1)=1 .
\end{gathered}
$$

Then we obtain from (38)

$$
\begin{gathered}
(P-1)\left|\tilde{U}^{\prime}\right\rangle=(P-1)(1+P) \mathcal{O}\left|\Psi_{i}\right\rangle+ \\
\left((P-1) P t G_{0}+(P-1)(1+P) V^{(1)} G_{0}\left(1+t G_{0}\right) \frac{1}{2} P(P-1)\right)\left|\tilde{U}^{\prime}\right\rangle,
\end{gathered}
$$

or with the definition

$$
(P-1)\left|\tilde{U}^{\prime}\right\rangle \equiv|\tilde{U}\rangle
$$

the following equation for $\tilde{U}$

$$
|\tilde{U}\rangle=(1+P) \mathcal{O}\left|\Psi_{i}\right\rangle+\left(t G_{0} P+\frac{1}{2}(P+1) V^{(1)} G_{0}\left(1+t G_{0}\right) P\right)|\tilde{U}\rangle .
$$

This integral equation is now suitable for numerical applications and provides according to (41) and (39) the nuclear matrix element

$$
N=\frac{1}{2}\left\langle\varphi_{0}\left|\left(1+t G_{0}\right) P\right| \tilde{U}\right\rangle
$$

In order to separate the contribution from the plane wave alone $\left(\left\langle\varphi_{0}\right|\right)$ and the symmetrized plane wave $\left(\left\langle\phi_{0}\right|=\left\langle\varphi_{0}\right|(1+P)\right)$ one can modify the driving term in (44) and solve the following equation for $|\tilde{\tilde{U}}\rangle$

$$
\begin{aligned}
|\tilde{\tilde{U}}\rangle & =\left[t G_{0}+\frac{1}{2}(P+1) V^{(1)} G_{0}\left(1+t G_{0}\right)\right](1+P) \mathcal{O}\left|\Psi_{i}\right\rangle \\
& +\left(t G_{0} P+\frac{1}{2}(P+1) V^{(1)} G_{0}\left(1+t G_{0}\right) P\right)|\tilde{\tilde{U}}\rangle
\end{aligned}
$$


With that auxiliary state $|\tilde{\tilde{U}}\rangle$ the amplitude $N$ reads now

$$
N=\left\langle\varphi_{0}\left|\left(1+t G_{0}\right)(1+P) \mathcal{O}\right| \Psi_{i}\right\rangle+\left\langle\varphi_{0}\left|\left(1+t G_{0}\right) P\right| \tilde{\tilde{U}}\right\rangle
$$

Dropping the second term and $t G_{0}$ in the first term in (47) one encounters two plane wave impulse approximations to the amplitude $N$

$$
N^{P W I A} \equiv\left\langle\varphi_{0}|\mathcal{O}| \Psi_{i}\right\rangle
$$

and

$$
N^{P W I A S} \equiv\left\langle\varphi_{0}|(1+P) \mathcal{O}| \Psi_{i}\right\rangle
$$

While in (48) the final state is antisymmetrized only in one pair, in (49) it is fully antisymmetrized. The verification of (46) and (47) requires straightforward algebra.

A completely alternative approach is based on two coupled Faddeev equations, again starting from (38). Defining

$$
\begin{aligned}
\left|U^{\prime}\right\rangle & \equiv t G_{0}\left|\tilde{U}^{\prime}\right\rangle \\
\left|U^{\prime \prime}\right\rangle & \equiv V^{(1)} G_{0}\left(t G_{0}+1\right)\left|\tilde{U}^{\prime}\right\rangle
\end{aligned}
$$

and

$$
|\chi\rangle \equiv(1+P) \mathcal{O}\left|\Psi_{i}\right\rangle
$$

one obviously obtains the set of coupled equations for $U^{\prime}$ and $U^{\prime \prime}$

$$
\begin{aligned}
\left|U^{\prime}\right\rangle & =t G_{0}|\chi\rangle+t G_{0} P\left|U^{\prime}\right\rangle+t G_{0}(1+P)\left|U^{\prime \prime}\right\rangle \\
\left|U^{\prime \prime}\right\rangle & =V^{(1)} G_{0}\left(1+t G_{0}\right)|\chi\rangle+V^{(1)} G_{0}\left(1+t G_{0}\right) P\left|U^{\prime}\right\rangle \\
& +V^{(1)} G_{0}\left(1+t G_{0}\right)(1+P)\left|U^{\prime \prime}\right\rangle .
\end{aligned}
$$

These three states (50), (51) and (52) sum up by definition to

$$
\left|\tilde{U}^{\prime}\right\rangle=|\chi\rangle+P\left|U^{\prime}\right\rangle+(1+P)\left|U^{\prime \prime}\right\rangle
$$

which determines according to (39) the breakup matrix element. Inserting the definition of ${ }^{(-)}\left\langle\varphi_{0}\right|$ and using (53) again, the breakup matrix element is easily turned into the simpler form

$$
N=\left\langle\varphi _ { 0 } \left|\left(|\chi\rangle+(1+P)\left(\left|U^{\prime}\right\rangle+\left|U^{\prime \prime}\right\rangle\right)\right) .\right.\right.
$$


For the pd breakup of ${ }^{3} \mathrm{He}$, the final channel state regarded up to now

$$
{ }^{(-)}\left\langle\varphi_{0}\right|=\left\langle\varphi_{0}\right|\left(1+t G_{0}\right) \equiv{ }_{a}^{(-)}\langle\vec{p} \vec{q}|
$$

has simply to be replaced by

$$
\left\langle\phi_{q}\right| \equiv\left\langle\varphi_{d}\right|\langle\vec{q}| .
$$

Thus the two-body scattering state ${ }_{a}^{(-)}\langle\vec{p}|$ turns into the deuteron state $\left\langle\varphi_{d}\right|$ and the pd breakup matrix element is given as

$$
N^{p d}=\frac{1}{2}\left\langle\phi_{q}|P| \tilde{U}\right\rangle,
$$

or

$$
N^{p d}=\left\langle\phi_{q}|(1+P) \mathcal{O}| \Psi_{i}\right\rangle+\left\langle\phi_{q}|P| \tilde{\tilde{U}}\right\rangle,
$$

if the auxiliary state $|\tilde{\tilde{U}}\rangle$ is employed.

If one uses the coupled set of equations, (53), the matrix element $N^{p d}$ will be

$$
N^{p d}=\left\langle\phi_{q} \mid \tilde{U}^{\prime}\right\rangle=\left\langle\phi _ { q } \left|\left(|\chi\rangle+P\left|U^{\prime}\right\rangle+(1+P)\left|U^{\prime \prime}\right\rangle\right)\right.\right. \text {. }
$$

We refrain to quote again the simpler equations given in [48-51, 73] valid for NN forces only. The more complex equations are necessary since for light nuclei $[26,74]$ and few-nucleon scattering processes [18] 3N forces are mandatory. In the context of effective field theory constrained by chiral symmetry NN and three- and more-nucleon forces are consistently linked to each other [75]. Applications in that framework to few-nucleon systems [76] definitely show that more than pairwise forces are acting and are clearly visible in the measured values of the observables (binding energies and scattering observables). This new approach grounded on effective field theory backs up the earlier results based on phenomenological forces which were constrained only by the one- $\pi$ exchange, that three-nucleon forces are necessary to describe the data.

The basic equations (44) or (53) are valid for electron induced reactions and for real photon induced processes as well. They only differ in the choice of the photon absorption operator $\mathcal{O}$ (see Section III).

In the case of nucleon-deuteron capture one can use time reversal invariance and evaluate the nuclear matrix element via $\mathrm{Nd}$ photodisintegration of the $3 \mathrm{~N}$ bound state as given in (58), (59) or (60). A more direct way is to choose the matrix element in the form

$$
N_{\text {capture }}=\left\langle\Psi|\mathcal{O}| \Psi_{i}^{(+)}\right\rangle,
$$


where $\left|\Psi_{i}^{(+)}\right\rangle$is the $\mathrm{Nd}$ scattering state with appropriately chosen initial state quantum numbers $i, \mathcal{O}$ a suitable operator depending on the final photon momentum, and $\langle\Psi|$ the $3 \mathrm{~N}$ bound state. Here we can use directly the Faddeev equation for the $3 \mathrm{~N}$ scattering state [71]. It corresponds to (29) and for the initial $\mathrm{Nd}$ channel is given as

$$
\left|\psi^{(+)}\right\rangle=\left|\phi_{i}\right\rangle+G_{0} t P\left|\psi^{(+)}\right\rangle+\left(1+t G_{0}\right) G_{0} V^{(1)}(1+P)\left|\psi^{(+)}\right\rangle .
$$

Here $\left|\phi_{i}\right\rangle \equiv\left|\phi_{q}\right\rangle$ with appropriate initial spin quantum numbers. The total scattering state is then

$$
\left|\Psi_{i}^{(+)}\right\rangle=(1+P)\left|\psi^{(+)}\right\rangle
$$

Let us define the amplitude $|T\rangle$ by

$$
\left|\psi^{(+)}\right\rangle=\left|\phi_{i}\right\rangle+G_{0}|T\rangle
$$

where $|T\rangle$ obeys the Faddeev-like equation

$$
|T\rangle=t P\left|\phi_{i}\right\rangle+\left(1+t G_{0}\right) V^{(1)}(1+P)\left|\phi_{i}\right\rangle+t P G_{0}|T\rangle+\left(1+t G_{0}\right) V^{(1)}(1+P)|T\rangle .
$$

It is this central equation (65) which we solve for 3N scattering [71]. Consequently the nuclear matrix element for $\mathrm{Nd}$ capture is obtained in the form

$$
N_{\text {capture }}=\left\langle\Psi|\mathcal{O}(1+P)| \phi_{i}\right\rangle+\left\langle\Psi\left|\mathcal{O}(1+P) G_{0}\right| T\right\rangle
$$

\section{CURRENT OPERATORS}

While the treatment of the interacting nucleons in the $3 \mathrm{~N}$ bound and scattering states is quite well established in the framework of the nonrelativistic Schrödinger equation, for the current operator, there is still quite some room for improvements. The current operator is a dynamical object containing in addition to a single nucleon term also the two- and threebody contributions, which are as complex as nuclear forces themselves. First considerations can be found in [77] and [78]. A very nice discussion and review is given in [26]. Earlier reviews for instance are [79] and [25]. Since our review does not focus on this issue, we will only briefly describe what underlies our applications.

One approach to include some of the many-body terms in the current, applied in the case

of photodisintegration (or Nd capture), is based on the old Siegert idea [80]. The way we 
use it is described in section IIIB. The other approach used for virtual and real photons is to link a certain subset of currents via the continuity equation to the NN force AV18, which has been phrased "model independent" in $[52,53]$. This is briefly reviewed in section III C. For additional currents not constrained by the continuity equation we refer the reader to [26]. We start with the single nucleon current in section III A.

\section{A. The single nucleon current}

We work in the Hamiltonian formalism and therefore the nucleons are on the mass shell. The standard single nucleon current at space-time point zero $j_{S N}^{\mu}(0)$ expressed in terms of the nucleon four momentum $p \equiv\left(p_{0}=\sqrt{m_{N}^{2}+\vec{p}^{2}}, \vec{p}\right)$ is

$$
j_{S N}^{\mu}(0)=\bar{u}\left(\vec{p}^{\prime} s^{\prime}\right)\left(\gamma^{\mu} F_{1}+i \sigma^{\mu \nu}\left(p^{\prime}-p\right)_{\nu} F_{2}\right) u(\vec{p} s)=\bar{u}\left(\vec{p}^{\prime} s^{\prime}\right)\left(G_{M} \gamma^{\mu}-F_{2}\left(p^{\prime}+p\right)^{\mu}\right) u(\vec{p} s)
$$

Here $u$ are Dirac spinors, $F_{1}\left(\left(p^{\prime}-p\right)^{2}\right)$ and $F_{2}\left(\left(p^{\prime}-p\right)^{2}\right)$ the Dirac and Pauli nucleon form factors, and $G_{M} \equiv F_{1}+2 m_{N} F_{2}$ the magnetic form factor of the nucleon. That fully relativistic form can be expressed as a four component $2 \times 2$ matrix operator $J^{\mu}\left(p^{\prime}, p\right)$ acting on Pauli spinors $\xi$ :

$$
j_{S N}^{\mu}(0)=\xi^{\dagger}\left(s^{\prime}\right) J^{\mu}\left(p^{\prime}, p\right) \xi(s) .
$$

With

$$
A=\sqrt{\frac{m_{N}}{p_{0}}} \sqrt{\frac{m_{N}}{p_{0}^{\prime}}} \sqrt{\frac{p_{0}^{\prime}+m_{N}}{2 m_{N}}} \sqrt{\frac{p_{0}+m_{N}}{2 m_{N}}}
$$

the components $J^{\mu}\left(p^{\prime}, p\right)$ are written as

$$
\begin{aligned}
J^{0}=A\left\{\left[G_{M}-F_{2}\left(p+p^{\prime}\right)^{0}\right]\right. & \left.+\left[G_{M}+F_{2}\left(p+p^{\prime}\right)^{0}\right] \frac{\vec{p}^{\prime} \cdot \vec{p}}{\left(p_{0}+m_{N}\right)\left(p_{0}^{\prime}+m_{N}\right)}\right\} \\
& +A\left[G_{M}+F_{2}\left(p+p^{\prime}\right)^{0}\right] \frac{i \vec{\sigma} \cdot\left(\vec{p}^{\prime} \times \vec{p}\right)}{\left(p_{0}+m_{N}\right)\left(p_{0}^{\prime}+m_{N}\right)}
\end{aligned}
$$

and

$$
\begin{array}{r}
J^{k}=-A F_{2}\left(1-\frac{\vec{p}^{\prime} \cdot \vec{p}}{\left(p_{0}+m_{N}\right)\left(p_{0}{ }^{\prime}+m_{N}\right)}\right)\left(p+p^{\prime}\right)^{k} \\
+A G_{M}\left(\frac{p^{k}}{p_{0}+m_{N}}+\frac{p^{\prime}}{p_{0}^{\prime}+m_{N}}\right) \\
+A F_{2} \frac{\left(p+p^{\prime}\right)^{k}}{\left(p_{0}+m_{N}\right)\left(p_{0}^{\prime}+m_{N}\right)} i \vec{\sigma} \cdot\left(\vec{p}^{\prime} \times \vec{p}\right) \\
+A G_{M}\left[\frac{1}{\left(p_{0}+m_{N}\right)} i(\vec{p} \times \vec{\sigma})^{k}+\frac{1}{\left(p_{0}^{\prime}+m_{N}\right)} i\left(\vec{\sigma} \times \vec{p}^{\prime}\right)^{k}\right] .
\end{array}
$$


This form will be used in section VI.

In the bulk of this review we apply only the nonrelativistic limit leading to the simple forms for the convection and spin current components:

$$
\vec{J}=F_{1} \frac{\vec{p}+\vec{p}^{\prime}}{2 m_{N}}+\frac{i}{2 m_{N}} G_{M} \vec{\sigma} \times\left(\vec{p}^{\prime}-\vec{p}\right) .
$$

The nucleon form factors $F_{1}$ and $F_{2}$, and thus $G_{M}$, are normalized for neutron and proton as

$$
\begin{gathered}
F_{1}^{n}(0)=0 \\
F_{1}^{p}(0)=1 \\
F_{2}^{n}(0)=-1.913 \frac{1}{2 m_{N}}=G_{M}^{n}(0) \frac{1}{2 m_{N}} \\
F_{2}^{p}(0)=1.793 \frac{1}{2 m_{N}}=G_{M}^{p}(0) \frac{1}{2 m_{N}}-\frac{1}{2 m_{N}} .
\end{gathered}
$$

In the case of the density component the leading term in the nonrelativistic limit

$$
J^{0}=F_{1}
$$

is very small for the neutron and therefore one generally adds the next order relativistic corrections, which are of the form $[81,82]$

$$
J^{0}=G_{E}\left(1-\frac{\vec{Q}^{2}}{8 m_{N}^{2}}\right)+i\left(2 G_{M}-G_{E}\right) \frac{\vec{\sigma} \cdot \vec{p}^{\prime} \times \vec{p}}{4 m_{N}^{2}}
$$

with the electric form factor

$$
G_{E} \equiv F_{1}+\frac{Q^{2}}{2 m_{N}} F_{2} \approx F_{1}-\frac{\vec{Q}^{2}}{2 m_{N}} F_{2}
$$

Due to formal reasons we use that form also for the proton. Here $Q=\left(Q_{0}, \vec{Q}\right) \equiv(\omega, \vec{Q})$ is the real or virtual photon four-momentum and $\vec{Q} \equiv \vec{p}^{\prime}-\vec{p}$.

In the case of the convection current in (72) some authors [26] replace $F_{1}$ by $G_{E}$ which adds some (not all) relativistic corrections of $O\left(\left(p / m_{N}\right)^{2}\right)$ on top of the leading order going with $F_{1}$. Once, however, $G_{E}$ is chosen for the density, then of course $G_{E}$ should also be used for the convection current due to current conservation.

The choice which underlies our nonrelativistic calculation here is $G_{E}$ for the density in lowest order and $G_{E}$ in the convection current instead of $F_{1}$ shown in (72). The spin current with $G_{M}$ is used as in (72). 
For the convenience of the reader we display the functional forms of the various nucleon form factors restricted to our momentum range in Figs. 4-5. We show theoretical predictions based on a dispersion theoretical analysis constrained by data [83]. Recent reviews on nucleon form factors can be found in [84].

In the nonrelativistic regime we choose for virtual photons $\vec{Q}^{2}$ to be the argument of the nucleon electromagnetic form factors. In case of real photons we put this argument to be zero since for our momentum range $\vec{Q}^{2}$ is anyway very small.

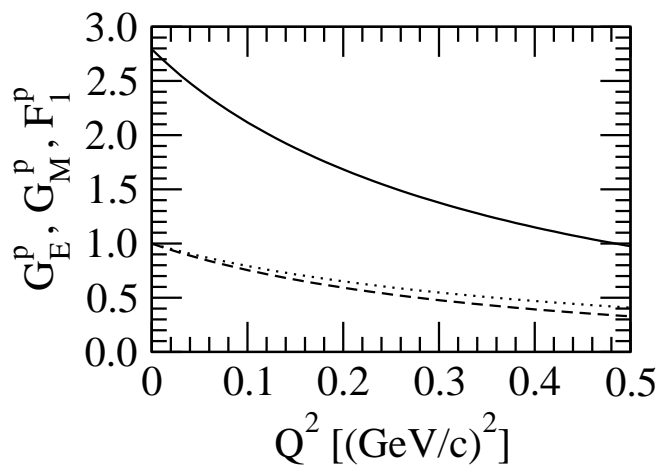

FIG. 4: The electromagnetic proton form factors $G_{E}^{p}$ (dashed line) and $G_{M}^{p}$ (solid line) from [83] used in our calculations for this review as a function of the four-momentum transfer squared $Q^{2}$. For the orientation of the reader $F_{1}^{p}$ (dotted line) is also shown.

\section{B. The Siegert approach}

Let $\vec{\epsilon}_{\xi}(\vec{Q})$ be the spherical component of the photon polarization vector for a photon with three-momentum $\vec{Q}$ and $\vec{j}(0)$ the nuclear current operator at space-time point zero. Then the nuclear matrix element for photodisintegration is written as

$$
N_{\xi}(\vec{Q})=\left\langle\vec{P}^{\prime} \Psi_{f}^{(-)}\left|\vec{\epsilon}_{\xi}(\vec{Q}) \cdot \vec{j}(0)\right| \Psi_{i} \vec{P}\right\rangle \equiv \vec{\epsilon}_{\xi}(\vec{Q}) \cdot \vec{I}(\vec{Q})
$$

As before $\left|\Psi_{i}\right\rangle$ and $\left\langle\Psi_{f}^{(-)}\right|$are the internal $3 \mathrm{~N}$ bound and scattering states and we added the dependence on the total initial and final 3N momenta. Clearly $\vec{P}^{\prime}=\vec{P}+\vec{Q}$ as expressed in the overall $\delta$-function of four-momentum conservation. This $\delta$-function is taken care of in the evaluation of the observables. Each component of the pure nuclear matrix element 

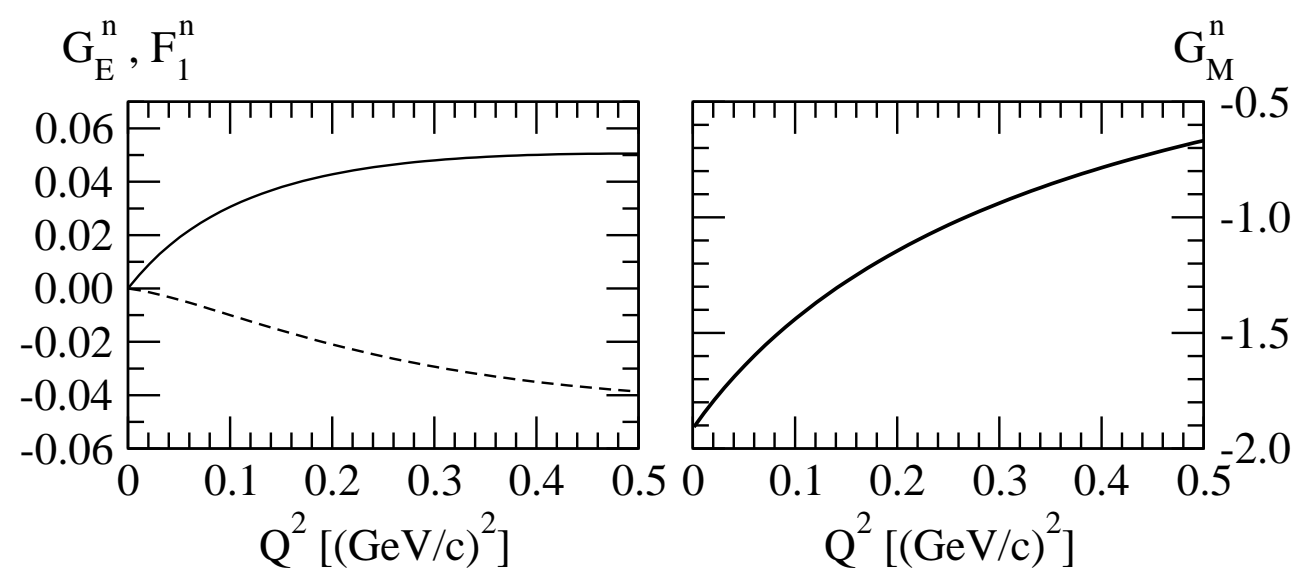

FIG. 5: The electromagnetic neutron form factors $G_{E}^{n}$ and $G_{M}^{n}$ (solid lines) from [83] used in our calculations for this review as functions of the four-momentum transfer squared $Q^{2}$. For the orientation of the reader $F_{1}^{n}$ (dashed line) is also shown.

$\vec{I}(\vec{Q})$ can be expanded into spherical harmonics

$$
I_{k}(\vec{Q})=\sum_{l m} Y_{l m}^{*}(\hat{Q}) \int d \hat{Q}^{\prime} Y_{l m}\left(\hat{Q}^{\prime}\right) I_{k}\left(|\vec{Q}| \hat{Q}^{\prime}\right) .
$$

Here and throughout the paper the "hat" notation stands sometimes for a unit vector. Further the polarization vector $\vec{\epsilon}_{\xi}(\vec{Q})$ for a photon with momentum in $\hat{Q}$-direction is related to the photon polarization vector $\vec{\epsilon}_{\xi}(\hat{z})$ for a photon with momentum in z-direction by a rotation. Since $\vec{\epsilon}_{\xi}$ is a rank one object, one has

$$
\vec{\epsilon}_{\xi}(\hat{Q})=\sum_{\xi^{\prime}} D_{\xi^{\prime} \xi}^{1}(\hat{Q}) \vec{\epsilon}_{\xi^{\prime}}(\hat{z})
$$

and using the Clebsch-Gordan coefficients we combine $\vec{\epsilon}_{\xi^{\prime}}(\hat{z})$ with $Y_{l m}\left(\hat{Q}^{\prime}\right)$ to vector spherical harmonics [85]

$$
\vec{\epsilon}_{\xi^{\prime}}(\hat{z}) Y_{l m}\left(\hat{Q}^{\prime}\right) \equiv \sum_{J \geq 1} C\left(l 1 J ; 0, \xi^{\prime}, \xi^{\prime}\right) \vec{Y}_{l 1 J}^{\xi^{\prime}}\left(\hat{Q}^{\prime}\right)
$$

Thus altogether we obtain

$$
N_{\xi}(\vec{Q})=\sum_{l m} Y_{l m}^{*}(\hat{Q}) \sum_{\xi^{\prime}} D_{\xi^{\prime} \xi}^{1}(\hat{Q}) \int d \hat{Q}^{\prime} \sum_{J \geq 1} C\left(l 1 J ; 0, \xi^{\prime}, \xi^{\prime}\right) \vec{Y}_{l 1 J}^{\xi^{\prime}}\left(\hat{Q}^{\prime}\right) \cdot \vec{I}\left(|\vec{Q}| \hat{Q}^{\prime}\right) .
$$

Now we can use [86]

$$
Y_{l m}^{*}(\hat{Q})=\sqrt{\frac{2 l+1}{4 \pi}} D_{m 0}^{l}(\hat{Q})
$$


as well as

$$
\sum_{m} C\left(l_{1} l_{2} L ; m, M-m, M\right) D_{m m_{1}}^{l_{1}} D_{M-m m_{2}}^{l_{2}}=C\left(l_{1} l_{2} L ; m_{1}, m_{2}, m_{1}+m_{2}\right) D_{M m_{1}+m_{2}}^{L}
$$

and obtain after rearranging the summation over the magnetic quantum numbers

$$
N_{\xi}(\vec{Q})=\sum_{J \geq 1} \sum_{M=-J}^{J} D_{M \xi}^{J}(\hat{Q}) \sum_{l=J, J \pm 1} \sqrt{\frac{2 l+1}{4 \pi}} C(l 1 J ; 0, \xi, \xi) \int d \hat{Q}^{\prime} \vec{Y}_{l 1 J}^{M}\left(\hat{Q}^{\prime}\right) \cdot \vec{I}\left(|\vec{Q}| \hat{Q}^{\prime}\right) .
$$

This nicely shows the dependence on the photon direction together with projections of the pure nuclear matrix element into the vector spherical harmonics. The latter ones are conventionally called the electric and magnetic multipole elements. Inserting the ClebschGordan coefficients one defines

$$
T_{J M}^{e l}(|\vec{Q}|) \equiv-\frac{1}{4 \pi} \int d \hat{Q}^{\prime}\left\{\sqrt{\frac{J}{2 J+1}} \vec{Y}_{J+11 J}^{M}\left(\hat{Q}^{\prime}\right)+\sqrt{\frac{J+1}{2 J+1}} \vec{Y}_{J-11 J}^{M}\left(\hat{Q}^{\prime}\right)\right\} \cdot \vec{I}\left(|\vec{Q}| \hat{Q}^{\prime}\right)
$$

and

$$
T_{J M}^{m a g}(|\vec{Q}|) \equiv \frac{1}{4 \pi} \int d \hat{Q}^{\prime} \vec{Y}_{J 1 J}^{M}\left(\hat{Q}^{\prime}\right) \cdot \vec{I}\left(|\vec{Q}| \hat{Q}^{\prime}\right)
$$

This leads then to

$$
N_{\xi}(\vec{Q})=-\sqrt{2 \pi} \sum_{J \geq 1} \sqrt{2 J+1} \sum_{M=-J}^{J} D_{M \xi}^{J}(\hat{Q})\left\{ \pm T_{J M}^{m a g}(|\vec{Q}|)+T_{J M}^{e l}(|\vec{Q}|)\right\},
$$

where $( \pm)$ refers to $\xi= \pm 1$.

Now the identity [85]

$$
\hat{Q} Y_{J M}(\hat{Q})=-\sqrt{\frac{J+1}{2 J+1}} \vec{Y}_{J+11 J}^{M}(\hat{Q})+\sqrt{\frac{J}{2 J+1}} \vec{Y}_{J-11 J}^{M}(\hat{Q})
$$

applied in (88) allows us to express $\vec{Y}_{J-11 J}^{M}(\hat{Q})$ in terms of a vector spherical harmonics with an orbital part larger by 2 and by $\hat{Q} Y_{J M}(\hat{Q})$. This leads to the expression $\hat{Q} \cdot \vec{I}(\vec{Q})$ which occurs in the continuity equation for the electromagnetic current matrix element:

$$
\vec{Q} \cdot \vec{I}(\vec{Q})=\left\langle\vec{P}^{\prime} \Psi_{f}^{(-)}|[H, \hat{\rho}(0)]| \Psi_{i} \vec{P}\right\rangle=\left(E_{f}-E_{i}\right)\left\langle\vec{P}^{\prime} \Psi_{f}^{(-)}|\hat{\rho}(0)| \Psi_{i} \vec{P}\right\rangle .
$$

Here $\hat{\rho}(0) \equiv j^{0}(0)$ is the density operator. Because of energy conservation, $E_{f}-E_{i}=\omega$, the photon energy $\omega=|\vec{Q}|$. Thus with

$$
\rho(\vec{Q}) \equiv\left\langle\vec{P}^{\prime} \Psi_{f}^{(-)}|\hat{\rho}(0)| \Psi_{i} \vec{P}\right\rangle,
$$


we end up with the electric multipole term in the form

$$
\begin{array}{r}
T_{J M}^{e l}(|\vec{Q}|)=-\frac{1}{4 \pi} \int d \hat{Q}^{\prime}\left\{\sqrt{\frac{2 J+1}{J}} \vec{Y}_{J+11 J}^{M}\left(\hat{Q}^{\prime}\right) \cdot \vec{I}\left(|\vec{Q}| \hat{Q}^{\prime}\right)\right. \\
\left.+\sqrt{\frac{J+1}{J}} Y_{J M}\left(\hat{Q}^{\prime}\right) \rho\left(|\vec{Q}| \hat{Q}^{\prime}\right)\right\} .
\end{array}
$$

Summarizing, one finds that by using the identity among vector spherical harmonics (91) together with the continuity equation it is possible to replace a part of the current

matrix element $\vec{I}(\vec{Q})$ by the density matrix element $\rho(\vec{Q})$ and higher multipole contributions. Because the matrix element $\rho(\vec{Q})$ receives two-body contributions only at a higher order in a $p / m$ expansion than the current matrix element [26], one can expect that the form (94) for the electric multipole contribution is a better approximation than (88) even when only the single nucleon density operator is used. The higher multipole term in (94) is usually neglected in the literature, but not in our applications. We also do not approximate (94) in a long wave length limit. We take zero as arguments in the nucleon electromagnetic form factors for all processes with real photons. In the nonrelativistic framework one should rather take the photon three-momentum squared. The results based on these two approaches are practically indistinguishable for low energies but for $\omega=140 \mathrm{MeV} / \mathrm{c}$ they lead to differences in the cross section up to about $8 \%$. For polarization observables these changes are much smaller (about 1\%).

\section{C. $\pi$ and $\rho$-like meson exchange currents}

The seminal papers [87] introduced $\pi$ - and heavy-meson exchange current (MEC) operators satisfying the continuity equation with meson-exchange interactions. But of course they violate the continuity equation in relation to phenomenological high precision NN forces like AV18, which we employ. Thus we follow a recipe adapted to phenomenological NN forces. For the sake of completeness we briefly sketch the derivation formulated in $[52,53,88]$. Equivalent proposals have been given in [55] and [56].

High accuracy NN forces like AV18 are not formed in a pure meson exchange picture but, except for the long range one- $\pi$ exchange, they contain a phenomenological structure dependent on a number of parameters. Nevertheless the spin-isospin structure occurring in a proper one- $\pi$ and one- $\rho$ exchange is present. For the isovector exchanges this is the 
$\vec{\tau}(1) \cdot \vec{\tau}(2)$ isospin operator. Also there occur the spin-spin and the tensor operators. In addition for the $\rho$-exchange there is a pure central term. Therefore, this part of a local NN force reads in momentum space

$$
V=\sum_{t=0}^{1} v_{S S}^{t}(k) \tilde{\Omega}_{S S}(\vec{k}) P_{t}+\sum_{t=0}^{1} v_{T}^{t}(k) \tilde{\Omega}_{T}(\vec{k}) P_{t}+\sum_{t=0}^{1} v_{C}^{t}(k) \tilde{\Omega}_{C} P_{t},
$$

with the isospin projection operators $P_{t=0}=\frac{1}{4}(1-\vec{\tau}(1) \cdot \vec{\tau}(2))$ and $P_{t=1}=\frac{1}{4}(3+\vec{\tau}(1) \cdot \vec{\tau}(2))$, and the spin operators

$$
\begin{gathered}
\tilde{\Omega}_{S S}(\vec{k})=\vec{k}^{2} \vec{\sigma}(1) \cdot \vec{\sigma}(2), \\
\tilde{\Omega}_{T}(\vec{k})=\vec{k}^{2} \vec{\sigma}(1) \cdot \vec{\sigma}(2)-3 \vec{\sigma}(1) \cdot \vec{k} \vec{\sigma}(2) \cdot \vec{k}, \\
\tilde{\Omega}_{C}=1 .
\end{gathered}
$$

In $(95) v_{S S}^{t}(k), v_{T}^{t}(k)$ and the central piece $v_{C}^{t}(k)$ are radial functions depending on $|\vec{k}| \equiv \mid$ $\vec{p}^{\prime}-\vec{p} \mid$, where $\vec{p}^{\prime}$ and $\vec{p}$ are the final and initial relative two-nucleon momenta.

Separating the term with $\vec{\tau}(1) \cdot \vec{\tau}(2)$ one obtains

$$
V \rightarrow v_{1} \vec{\tau}(1) \cdot \vec{\tau}(2) \equiv \vec{\tau}(1) \cdot \vec{\tau}(2)\left(v_{S S}(k) \tilde{\Omega}_{S S}(\vec{k})+v_{T}(k) \tilde{\Omega}_{T}(\vec{k})+v_{C}(k) \tilde{\Omega}_{C}\right),
$$

with

$$
\begin{aligned}
v_{S S}(k) & \equiv \frac{1}{4}\left(v_{S S}^{t=1}(k)-v_{S S}^{t=0}(k)\right), \\
v_{T}(k) & \equiv \frac{1}{4}\left(v_{T}^{t=1}(k)-v_{T}^{t=0}(k)\right), \\
v_{C}(k) & \equiv \frac{1}{4}\left(v_{C}^{t=1}(k)-v_{C}^{t=0}(k)\right) .
\end{aligned}
$$

Now one assumes that $v_{S S}^{t}(k)$ and $v_{T}^{t}(k)$ are built up by the sum of $\pi$ - and $\rho$-like parts

$$
\begin{gathered}
v_{S S}^{t} \equiv v_{S S}^{\pi, t}+v_{S S}^{\rho, t}, \\
v_{T}^{t} \equiv v_{T}^{\pi, t}+v_{T}^{\rho, t},
\end{gathered}
$$

and that these parts obey the same relations which are valid for the true one- $\pi$ and one- $\rho$ exchange terms

$$
\begin{aligned}
& v_{S S}^{\pi, t}=-v_{T}^{\pi, t}, \\
& v_{S S}^{\rho, t}=2 v_{T}^{\rho, t} .
\end{aligned}
$$


All that taken together allows now to solve for the individual $\pi$ - and $\rho$-like parts contained in the potential $V$ in terms of $v_{S S}^{t}$ and $v_{T}^{t}$. According to (100)-(106) one obtains

$$
\begin{gathered}
v_{S S}^{t=1}-v_{S S}^{t=0}+v_{T}^{t=1}-v_{T}^{t=0}=\underbrace{\left(v_{S S}^{\pi, t=1}+v_{T}^{\pi, t=1}\right)}_{=0}-\underbrace{\left(v_{S S}^{\pi, t=0}+v_{T}^{\pi, t=0}\right)}_{=0} \\
+\left(v_{S S}^{\rho, t=1}+v_{T}^{\rho, t=1}\right)-\left(v_{S S}^{\rho, t=0}+v_{T}^{\rho, t=0}\right)=\frac{3}{2}\left(v_{S S}^{\rho, t=1}-v_{S S}^{\rho, t=0}\right) .
\end{gathered}
$$

Consequently, the $\rho$-like term of the potential in the form (99) is determined via the two functions $v_{S S}^{\rho}(k)$ and $v_{T}^{\rho}(k)$ given by

$$
v_{S S}^{\rho}(k) \equiv \frac{1}{4}\left(v_{S S}^{\rho, t=1}-v_{S S}^{\rho, t=0}\right)=\frac{1}{6}\left(v_{S S}^{t=1}+v_{T}^{t=1}-v_{S S}^{t=0}-v_{T}^{t=0}\right)=2 v_{T}^{\rho}(k),
$$

and the $\pi$-like part of the potential in the form (99) is determined by the two functions $v_{S S}^{\pi}$ and $v_{T}^{\pi}$ given by

$$
\begin{gathered}
v_{S S}^{\pi}(k)=v_{S S}(k)-v_{S S}^{\rho}(k), \\
v_{T}^{\pi}(k)=v_{T}(k)-v_{T}^{\rho}(k) .
\end{gathered}
$$

Also one assumes that $v_{C}(k)$ is a $\rho$-like object

$$
v_{C}^{\rho}(k)=v_{C}(k)
$$

In this manner the isospin dependent part (99) of the general potential (95) is separated into two parts, a $\pi$-like and $\rho$-like terms.

Now let us regard the continuity equation in the lowest nontrivial order of a $p / m$ expansion

$$
\left[V, j_{S N}^{0}(0)\right]=\left[\vec{P}, \vec{j}^{\text {exch }}(0)\right] .
$$

Here $V$ is a nonrelativistic NN force, like the lowest order one- $\pi$ or one- $\rho$ exchange potentials, $j_{S N}^{0}(0)$ is the single nucleon density operator taken at the space-time point $0, \vec{j}^{\text {exch }}(0)$ the related exchange current operator, and $\vec{P}$ the total two-nucleon momentum operator. Neglecting in (78) all terms except the first one the matrix element of $j_{S N}^{0}(0)$ is

$$
\left\langle\vec{p}_{i}^{\prime}\left|j_{S N}^{0}(0)\right| \vec{p}_{i}\right\rangle=G_{E}^{p}\left(\vec{p}_{i}^{\prime}-\vec{p}_{i}\right) \Pi^{p}+G_{E}^{n}\left(\vec{p}_{i}^{\prime}-\vec{p}_{i}\right) \Pi^{n}
$$

with $G_{E}^{p, n}$ and $\Pi^{p, n}$ the nucleon Sachs form factors and the projection operators for the proton and neutron, respectively. Then the equation (112) is easily worked out in momentum space with the result

$$
\vec{Q} \cdot\left\langle\vec{p}_{1}{ }^{\prime} \vec{p}_{2}{ }^{\prime}\left|\vec{j}^{\text {exch }}(0)\right| \vec{p}_{1} \vec{p}_{2}\right\rangle=
$$




$$
\begin{gathered}
\left(V\left(\vec{p}^{\prime}, \frac{\vec{Q}}{2}+\vec{p}\right)-V\left(\vec{p}^{\prime}-\frac{\vec{Q}}{2}, \vec{p}\right)\right)\left(G_{E}^{p}(\vec{Q}) \Pi^{p}(1)+G_{E}^{n}(\vec{Q}) \Pi^{n}(1)\right) \\
+\left(\quad V\left(\vec{p}^{\prime},-\frac{\vec{Q}}{2}+\vec{p}\right)-V\left(\vec{p}^{\prime}+\frac{\vec{Q}}{2}, \vec{p}\right)\right)\left(G_{E}^{p}(\vec{Q}) \Pi^{p}(2)+G_{E}^{n}(\vec{Q}) \Pi^{n}(2)\right) \\
+\quad i \quad\left(v_{1}\left(\vec{p}^{\prime}-\frac{\vec{Q}}{2}, \vec{p}\right)-v_{1}\left(\vec{p}^{\prime}+\frac{\vec{Q}}{2}, \vec{p}\right)\right)\left(G_{E}^{p}(\vec{Q})-G_{E}^{n}(\vec{Q})\right)(\vec{\tau}(1) \times \vec{\tau}(2))_{3} .
\end{gathered}
$$

For $V$ we assumed the form

$$
V\left(\vec{p}^{\prime}, \vec{p}\right)=v_{0}\left(\vec{p}^{\prime}, \vec{p}\right)+v_{1}\left(\vec{p}^{\prime}, \vec{p}\right) \vec{\tau}(1) \cdot \vec{\tau}(2)
$$

in terms of two-nucleon relative momenta. Further, having photon absorption in mind, $\vec{Q} \equiv \vec{P}^{\prime}-\vec{P}$ is the photon momentum.

For a purely local potential the first two terms on the right-hand-side of (114) vanish. For a pure one- $\pi$ exchange potential

$$
V_{\pi}=v_{\pi}(k) \vec{\sigma}(1) \cdot \vec{k} \vec{\sigma}(2) \cdot \vec{k} \vec{\tau}(1) \cdot \vec{\tau}(2)
$$

with

$$
v_{\pi}(k)=-\frac{f_{\pi N N}^{2}}{m_{\pi}^{2}} \frac{1}{m_{\pi}^{2}+k^{2}},
$$

a simple algebra employing (114) leads to the well known pure pionic exchange current [89]

$$
\begin{array}{r}
\vec{j}_{\pi}^{\text {exch }}\left(\vec{k}_{1}, \vec{k}_{2}\right)=i\left(G_{E}^{p}(\vec{Q})-G_{E}^{n}(\vec{Q})\right)(\vec{\tau}(1) \times \vec{\tau}(2))_{3} \\
\left(\vec{\sigma}(2) \vec{\sigma}(1) \cdot \vec{k}_{1} v_{\pi}\left(k_{1}\right)-\vec{\sigma}(1) \vec{\sigma}(2) \cdot \vec{k}_{2} v_{\pi}\left(k_{2}\right)\right. \\
\left.+\frac{\vec{k}_{1}-\vec{k}_{2}}{k_{1}^{2}-k_{2}^{2}}\left(v_{\pi}\left(k_{2}\right)-v_{\pi}\left(k_{1}\right)\right) \vec{\sigma}(1) \cdot \vec{k}_{1} \vec{\sigma}(2) \cdot \vec{k}_{2}\right) .
\end{array}
$$

The momentum $\vec{k}_{i} \equiv \vec{p}_{i}^{\prime}-\vec{p}_{i}$ is the momentum transferred to the nucleon $i$.

Similarly for the pure one- $\rho$ exchange potential

$$
V_{\rho}=v_{\rho}(k)(\vec{\sigma}(1) \times \vec{k}) \cdot(\vec{\sigma}(2) \times \vec{k})+v_{\rho}^{S}(k)
$$

with

$$
v_{\rho}(k)=-\left(\frac{g_{\rho N N}}{2 M}\right)^{2} \frac{(1+\kappa)^{2}}{m_{\rho}^{2}+k^{2}},
$$

and

$$
v_{\rho}^{S}(k)=g_{\rho N N}^{2} \frac{1}{m_{\rho}^{2}+k^{2}},
$$


one obtains going through the corresponding algebra

$$
\begin{array}{r}
\vec{j}_{\rho}^{\text {exch } \prime}=i\left(G_{E}^{p}(\vec{Q})-G_{E}^{n}(\vec{Q})\right)(\vec{\tau}(1) \times \vec{\tau}(2))_{3}\left[\frac{\vec{k}_{1}-\vec{k}_{2}}{k_{1}^{2}-k_{2}^{2}}\left(v_{\rho}^{S}\left(k_{2}\right)-v_{\rho}^{S}\left(k_{1}\right)\right)\right. \\
-\left(v_{\rho}\left(k_{2}\right) \vec{\sigma}(1) \times\left(\vec{\sigma}(2) \times \vec{k}_{2}\right)-v_{\rho}\left(k_{1}\right) \vec{\sigma}(2) \times\left(\vec{\sigma}(1) \times \vec{k}_{1}\right)\right) \\
\left.-\frac{v_{\rho}\left(k_{2}\right)-v_{\rho}\left(k_{1}\right)}{k_{1}^{2}-k_{2}^{2}}\left(\vec{\sigma}(1) \times \vec{k}_{1}\right) \cdot\left(\vec{\sigma}(2) \times \vec{k}_{2}\right)\left(\vec{k}_{1}-\vec{k}_{2}\right)\right] .
\end{array}
$$

That extraction of the two-body currents $\vec{j}^{\text {exch }}$ from the continuity equation obviously can determine only the longitudinal (direction $\hat{Q}$ ) part of $\vec{j}$ exch and in the case of the $\rho$-exchange in fact one piece required by the underlying Lagrangian is missing. The full expression is

$$
\begin{gathered}
\vec{j}_{\rho}^{\text {exch }}=\vec{j}_{\rho}^{\text {exch }}-i\left(G_{E}^{p}(\vec{Q})-G_{E}^{n}(\vec{Q})\right)(\vec{\tau}(1) \times \vec{\tau}(2))_{3} \\
\frac{v_{\rho}\left(k_{2}\right)-v_{\rho}\left(k_{1}\right)}{k_{1}^{2}-k_{2}^{2}}\left(\vec{\sigma}(2) \cdot\left(\vec{k}_{1} \times \vec{k}_{2}\right)\left(\vec{\sigma}(1) \times \vec{k}_{1}\right)+\vec{\sigma}(1) \cdot\left(\vec{k}_{1} \times \vec{k}_{2}\right)\left(\vec{\sigma}(2) \times \vec{k}_{2}\right)\right) .
\end{gathered}
$$

In (120)-(121) $g_{\rho N N}$ and $\kappa$ are the vector and tensor coupling constants and $m_{\rho}$ is the $\rho$-mass. This agrees with the expressions given in $[53,88]$.

Putting now the $\pi$-like part of the phenomenological potential $v_{1}$ in (99) and (109)-(110) into the form (116) of the pure pion exchange one finds the correspondence

$$
-3 v_{T}^{\pi}(k) \hat{=} v_{\pi}(k)
$$

This leads to the idea proposed in $[52,53,88]$ to replace $v_{\pi}(k)$ in $(118)$ by $-3 v_{T}^{\pi}(k)$ determined via (109)-(110) from the phenomenological potential (95). In this manner one arrives at the $\pi$-like exchange current which together with the $\pi$-like part of the force fulfills the continuity equation by construction.

Similarly putting the $\rho$-like part of the phenomenological potential in (99) and (108) into the form (119) of the pure $\rho$-exchange leads to the correspondence

$$
v_{T}^{\rho}(k) \hat{=} v_{\rho}(k),
$$

and

$$
v_{C}^{\rho}(k) \hat{=} v_{\rho}^{S}(k) .
$$

Therefore one replaces $v_{\rho}(k)$ in $(122)-(123)$ by $v_{T}^{\rho}(k)$ given in (108) and $v_{\rho}^{S}(k)$ in (122) by $v_{C}^{\rho}(k)$ given in (111). This leads to the $\rho$-like exchange current which again together with the $\rho$-like part of the force fulfills the continuity equation exactly. 
It remains to provide the forms of $v_{S S}^{t}(k), v_{T}^{t}(k)$, and $v_{C}^{t}(k)$ belonging to the local NN force

$$
V=\sum_{t=0}^{1} v_{S S}^{t}(r) \vec{\sigma}_{1} \cdot \vec{\sigma}_{2} P_{t}+\sum_{t=0}^{1} v_{T}^{t}(r)\left(3 \vec{\sigma}_{1} \cdot \vec{r} \vec{\sigma}_{2} \cdot \vec{r}-\vec{\sigma}_{1} \cdot \vec{\sigma}_{2}\right) P_{t}+\sum_{t=0}^{1} v_{C}^{t}(r) P_{t} .
$$

The Fourier transform of (127) results in

$$
\begin{gathered}
v_{T}^{t}(k)=\frac{4 \pi}{k^{2}} \int_{0}^{\infty} d r r^{2} j_{2}(k r) v_{T}^{t}(r), \\
v_{S S}^{t}(k)=\frac{4 \pi}{k^{2}} \int_{0}^{\infty} d r r^{2}\left[j_{0}(k r)-1\right] v_{S S}^{t}(r), \\
v_{C}^{t}(k)=4 \pi \int_{0}^{\infty} d r r^{2} j_{0}(k r) v_{C}^{t}(r) .
\end{gathered}
$$

The bracket $\left[j_{0}(k r)-1\right]$ in $(129)$ guarantees that the volume integral related to $v_{S S}^{t}(k)$ vanishes, like for the pure one- $\pi$ exchange.

Clearly there are additional two-body currents related to spin-orbit NN interactions and other momentum dependences in the NN force AV18. They have been considered in [90] and [91], and appear in general to be of less quantitative importance. The purely transverse currents are of course not constrained by the continuity equation. Among them $\rho \pi \gamma_{-}, \omega \pi \gamma_{-}$ and $\Delta$ - currents have been considered and we refer the reader to [26] and references therein for their discussion. Again they appear to be less important for low energy physics [26]. Based on current insights they are clearly strongly model dependent.

Quite recently $[92,93]$ currents have been constructed, which exactly fulfill the current continuity equation with the AV18 NN force in combination with the UrbanaIX 3NF. The authors follow the steps using minimal substitutions as outlaid in [94, 95]. The first observation on those steps is that $\vec{\tau}_{i} \cdot \vec{\tau}_{j}$ can be replaced by

$$
\vec{\tau}_{i} \cdot \vec{\tau}_{j}=-1-\left(1+\vec{\sigma}_{i} \cdot \vec{\sigma}_{j}\right) P^{\text {space }}(i, j)
$$

when applied to an antisymmetric wave function. Thereby the operator $P^{\text {space }}(i, j)$ exchanges the positions of particles $i$ and $j$. The key point is then that $P^{\text {space }}(i, j)$ can be expressed in terms of momentum operators as $[94,96]$

$$
P^{\text {space }}(i, j)=e^{\vec{r}_{j i} \cdot \vec{\nabla}_{i}+\vec{r}_{i j} \cdot \vec{\nabla}_{j}},
$$


where the $\vec{\nabla}$-operators do not act on the pair distances $\vec{r}_{i j}=-\vec{r}_{j i}$. In this form one can perform a minimal substitution to couple to an electromagnetic field $\vec{A}(\vec{r})$. This leads to an expression of the form

$$
P(\vec{r})=e^{\vec{a} \cdot \vec{\nabla}+g(\vec{r})}
$$

which can be expressed as

$$
P(\vec{r})=e^{\frac{1}{a} \int_{\vec{r}}^{\vec{r}+\vec{a}} d \vec{s} g(\vec{s})} e^{\vec{a} \cdot \vec{\nabla}}
$$

with a line integral along the straight line between the positions $\vec{r}$ and $\vec{r}+\vec{a}$. For the application to the NN force AV18 and the 3NF UrbanaIX we refer the reader to the very recent paper [93]. Here, low energy electronuclear observables, nd and pd radiative capture reactions and magnetic form factors of ${ }^{3} \mathrm{He}$ and ${ }^{3} \mathrm{H}$ are calculated. Comparative studies of new and old current models are performed. It turns out that three-body currents give small but significant contributions to some of the very low energy observables. For detailed information see [92, 93].

The interesting issue of modifications for the absorption mechanism of a photon on hadrons in nuclear medium is addressed in [97, 98]. 


\section{THE OBSERVABLES}

Knowing the nuclear matrix elements for electron scattering on ${ }^{3} \mathrm{He}\left({ }^{3} \mathrm{H}\right)$ the step to the rich variety of observables based on the one-photon exchange is standard. Thereby it is assumed that the initial and final nuclear states are eigenstates of the hadronic fourmomentum operator. This leads to the $S$-matrix element

$$
S_{f i}=i(2 \pi)^{4} \delta\left(k^{\prime}-k+P_{f}-P_{i}\right) \frac{e^{2}}{Q^{2}} L_{\mu} N^{\mu}
$$

where $k\left(k^{\prime}\right), P_{i}\left(P_{f}\right)$ are the initial (final) electron and nuclear four momenta,

$$
Q=k-k^{\prime}=P_{f}-P_{i}
$$

the photon four momentum, and

$$
\begin{gathered}
N^{\mu} \equiv\left\langle f\left|\frac{1}{e} j_{\text {hadron }}^{\mu}(0)\right| i\right\rangle \\
L^{\mu} \equiv\left\langle k^{\prime} s^{\prime}\left|-\frac{1}{e} j_{\text {electron }}^{\mu}(0)\right| k s\right\rangle
\end{gathered}
$$

the hadronic and electronic matrix elements. In a nonrelativistic treatment which we pursue, this underlying property of the hadronic states to be eigenstates of the hadronic fourmomentum operator $\hat{P}^{\mu}$

$$
\hat{P}^{\mu}|i, f\rangle=P_{i, f}^{\mu}|i, f\rangle
$$

is of course not fulfilled but in the derivation for the expression of the observables we nevertheless assume this to be true.

The cross section for the transition into the final states spanned by $d f$ reads

$$
d \sigma=(2 \pi)^{4} \delta\left(k^{\prime}-k+P_{f}-P_{i}\right) \frac{e^{4}}{Q^{4}}\left(L_{\mu} L_{\nu}^{*}\right)\left(N^{\mu} N^{\nu *}\right) d f .
$$

For an initially polarized electron with helicity $h$ one obtains by well known steps

$$
L_{\mu} L_{\nu}^{*}=\frac{1}{2 k_{0}^{\prime} k_{0}} \frac{1}{(2 \pi)^{6}}\left(k_{\mu} k_{\nu}^{\prime}+k_{\nu} k_{\mu}^{\prime}-g_{\mu \nu} k \cdot k^{\prime}-i h \epsilon_{\mu \nu \alpha \beta} k^{\alpha} k^{\prime \beta}\right) .
$$

Further we regard ultrarelativistic electrons $\left(m_{e} \rightarrow 0\right)$ and use current conservation in the form

$$
Q_{0} N^{0}-\vec{Q} \cdot \vec{N}=0
$$


Thus we can drop some terms in (140) and put

$$
L_{\mu} L_{\nu}^{*} \rightarrow \frac{1}{4 k_{0}^{\prime} k_{0}} \frac{1}{(2 \pi)^{6}}\left(K_{\mu} K_{\nu}+g_{\mu \nu} Q^{2}-2 i h \epsilon_{\mu \nu \alpha \beta} k^{\alpha} k^{\prime \beta}\right) \equiv \frac{1}{(2 \pi)^{6}} l_{\mu \nu}
$$

with

$$
K \equiv k+k^{\prime}
$$

The contraction with $N^{\mu} N^{\nu *}$ is a quite tedious step. Starting from

$$
l_{\mu \nu} N^{\mu} N^{\nu *}=\frac{1}{4 k_{0}^{\prime} k_{0}}\left(K \cdot N K \cdot N^{*}+Q^{2} N \cdot N^{*}-2 i h \epsilon_{\mu \nu \alpha \beta} k^{\alpha} k^{\prime \beta} N^{\mu} N^{\nu *}\right)
$$

one uses spherical unit vectors $\hat{e}_{\mu}$ to represent the space part of $N^{\mu}$ as

$$
\vec{N}=\hat{e}_{1}^{*} N_{1}+\hat{e}_{-1}^{*} N_{-1}+\hat{e}_{0} N_{0}
$$

The assumed current conservation (141), another property of the hadronic dynamics which is not always exactly fulfilled in the present day practice, allows to eliminate the component of $\vec{N}$ along $\vec{Q}$ in favor of $N_{0}$ :

$$
\hat{Q} \cdot \vec{N}=\frac{Q_{0}}{|\vec{Q}|} N_{0}
$$

From now on we shall choose the $z$-direction to be the direction of $\vec{Q}$. In rewriting (144) in terms of $N_{ \pm 1}$ and $N_{0}$ only the kinematical relation (135) is used. Further it is convenient to choose the plane spanned by $\vec{k}$ and $\vec{k}^{\prime}$ to coincide with the $x-z$ plane and to choose the positive $x$-direction such that $\left(k+k^{\prime}\right)_{x} \geq 0$. Then one obtains [99]

$$
\begin{array}{r}
d \sigma=(2 \pi)^{4} \delta\left(P_{f}-P_{i}-Q\right) \frac{e^{4}}{\left(Q^{2}\right)^{2}} \cos ^{2} \frac{\vartheta}{2} d f \\
{\left[v_{L} R_{L}+v_{T} R_{T}+v_{T T} R_{T T}+v_{T L} R_{T L}+h\left(v_{T^{\prime}} R_{T^{\prime}}+v_{T L^{\prime}} R_{T L^{\prime}}\right)\right]}
\end{array}
$$

where the purely kinematical functions $v$ are given in terms of electron properties only $(\vartheta$ is the laboratory electron scattering angle)

$$
\begin{array}{r}
v_{L}=\frac{\left(Q^{2}\right)^{2}}{\left(\vec{Q}^{2}\right)^{2}} \\
v_{T}=-\frac{1}{2} \frac{Q^{2}}{\vec{Q}^{2}}+\tan ^{2} \frac{\vartheta}{2} \\
v_{T T}=\frac{1}{2} \frac{Q^{2}}{\vec{Q}^{2}} \\
v_{T L}=\frac{1}{\sqrt{2}} \frac{Q^{2}}{\vec{Q}^{2}} \sqrt{-\frac{Q^{2}}{\vec{Q}^{2}}+\tan ^{2} \frac{\vartheta}{2}}
\end{array}
$$




$$
\begin{array}{r}
v_{T^{\prime}}=\sqrt{-\frac{Q^{2}}{\vec{Q}^{2}}+\tan ^{2} \frac{\vartheta}{2}} \tan \frac{\vartheta}{2} \\
v_{T L^{\prime}}=\frac{1}{\sqrt{2}} \frac{Q^{2}}{\vec{Q}^{2}} \tan \frac{\vartheta}{2} .
\end{array}
$$

The nuclear response functions are

$$
\begin{array}{r}
R_{L}=\left|N_{0}\right|^{2} \\
R_{T}=\left|N_{1}\right|^{2}+\left|N_{-1}\right|^{2} \\
R_{T T}=2 \Re\left(N_{1} N_{-1}^{*}\right) \\
R_{T L}=-2 \Re\left(N_{0}\left(N_{1}-N_{-1}\right)^{*}\right) \\
R_{T^{\prime}}=\left|N_{1}\right|^{2}-\left|N_{-1}\right|^{2} \\
R_{T L^{\prime}}=-2 \Re\left(N_{0}\left(N_{1}+N_{-1}\right)^{*}\right) .
\end{array}
$$

This is a general form for the cross section where the polarization of the hadronic states can still be chosen at will.

Let us first regard elastic scattering on ${ }^{3} \mathrm{He}$. Straightforward calculation of the phase space factor

$$
\rho \equiv \int \delta\left(P^{\prime}-P-Q\right) d f=\int \delta\left(P^{\prime}-P-Q\right) d \vec{P}^{\prime} d \vec{k}^{\prime}
$$

in the lab system leads to

$$
\rho=d \hat{k}^{\prime} \frac{E_{P^{\prime}}}{M} \frac{k_{0}^{\prime 2}}{1+\frac{k_{0}}{M}(1-\cos \vartheta)} .
$$

Here $M$ is the ${ }^{3}$ He mass, $E_{P^{\prime}}=\sqrt{M^{2}+\vec{P}^{\prime 2}}$, and

$$
k_{0}^{\prime}=\frac{k_{0}}{1+\frac{k_{0}}{M}(1-\cos \vartheta)} .
$$

Then one introduces the Mott cross section

$$
\sigma_{M o t t}=\frac{\alpha^{2} \cos ^{2} \frac{\vartheta}{2}}{4 k_{0}^{2} \sin ^{4} \frac{\vartheta}{2}}
$$

with $\alpha=\frac{e^{2}}{4 \pi} \approx \frac{1}{137}$ and obtains the differential cross section for unpolarized electron scattering on an unpolarized ${ }^{3} \mathrm{He}$ target state in the lab system

$$
\frac{d \sigma}{d \hat{k}^{\prime}}=\sigma_{M o t t} \frac{1}{1+\frac{k_{0}}{M}(1-\cos \vartheta)}\left[\frac{\left(Q^{2}\right)^{2}}{\left(\vec{Q}^{2}\right)^{2}} \mathcal{R}_{L}+\left(-\frac{1}{2} \frac{Q^{2}}{\vec{Q}^{2}}+\tan ^{2} \frac{\vartheta}{2}\right) \mathcal{R}_{T}\right]
$$


We defined longitudinal $\mathcal{R}_{L}$ and transversal $\mathcal{R}_{T}$ response functions, averaged over initial $(m)$ and summed over final $\left(m^{\prime}\right)$ spin magnetic quantum numbers

$$
\begin{gathered}
\mathcal{R}_{L}=(2 \pi)^{6} \frac{E_{P^{\prime}}}{M} \frac{1}{2} \sum_{m m^{\prime}}\left|N_{0}\right|^{2} \\
\mathcal{R}_{T}=(2 \pi)^{6} \frac{E_{P^{\prime}}}{M} \frac{1}{2} \sum_{m m^{\prime}}\left(\left|N_{1}\right|^{2}+\left|N_{-1}\right|^{2}\right) .
\end{gathered}
$$

This is usually written in terms of the charge and magnetic form factors [100]

$$
\begin{array}{r}
\frac{d \sigma}{d \hat{k}^{\prime}}=\sigma_{M o t t} \frac{Z^{2}}{1+\frac{k_{0}}{M}(1-\cos \vartheta)}\left[F_{C}^{2}\right. \\
\left.-\frac{Q^{2}}{4 M^{2}} F_{m}^{2}(1+\kappa)^{2}\left(1+2\left(1-\frac{Q^{2}}{4 M^{2}}\right) \tan ^{2} \frac{\vartheta}{2}\right)\right] \frac{1}{1-\frac{Q^{2}}{4 M^{2}}} .
\end{array}
$$

The form factors are normalized as

$$
\begin{gathered}
F_{C}\left(Q^{2}=0\right)=1 \\
F_{m}\left(Q^{2}=0\right)=1,
\end{gathered}
$$

such that $(1+\kappa)$ is the magnetic moment of ${ }^{3} \mathrm{He}$ in nuclear magnetons $\left(\frac{e}{2 m_{N}}\right)$ [101].

In contrast to (147) the response functions $R_{T T}$ and $R_{T L}$ do not show up in (157). The partial wave decomposition reveals [101] that they are zero in this case.

It is known that polarizing the initial electron or initial ${ }^{3} \mathrm{He}$ does not lead to new independent information [99].

Now we move on to inclusive scattering on ${ }^{3} \mathrm{He}$. Only the scattered electron is measured and one has to integrate over all final nucleon momenta. We choose the lab system and work nonrelativistically. The phase space factor in the pd channel is then

$$
\rho_{p d} \equiv \int \delta\left(\vec{P}^{\prime}-\vec{Q}\right) \delta\left(E_{d}+\frac{k_{d}^{2}}{4 m_{N}}+\frac{k_{p}^{2}}{2 m_{N}}-Q_{0}-E_{3} \mathrm{He}\right) d \vec{k}_{d} d \vec{k}_{p} d \vec{k}^{\prime}
$$

where $E_{d}\left(E_{3^{\mathrm{He}}}\right)$ is the (negative) deuteron $\left({ }^{3} \mathrm{He}\right)$ binding energy and $\vec{k}_{d}\left(\vec{k}_{p}\right)$ is the final deuteron (proton) momenta. Since the nuclear matrix element is evaluated in terms of Jacobi momenta it is convenient to change $\vec{k}_{d}$ and $\vec{k}_{p}$ to

$$
\begin{gathered}
\vec{q} \equiv \frac{2}{3}\left(\vec{k}_{p}-\frac{1}{2} \vec{k}_{d}\right) \\
\vec{P}^{\prime} \equiv \vec{k}_{p}+\vec{k}_{d} .
\end{gathered}
$$


This leads then to

$$
\rho_{p d}=d \hat{k}^{\prime} d \hat{q} \frac{2 m_{N}}{3}\left|\vec{q}_{0}\right|
$$

with

$$
\left|\vec{q}_{0}\right|=\sqrt{\frac{4 m_{N}}{3}\left(Q_{0}+E_{3 \mathrm{He}}-\frac{\vec{Q}^{2}}{6 m_{N}}-E_{d}\right)} .
$$

In the case of the $3 \mathrm{~N}$ breakup one introduces Jacobi momenta for both relative motions

$$
\begin{gathered}
\vec{p} \equiv \frac{1}{2}\left(\vec{k}_{2}-\vec{k}_{3}\right) \\
\vec{q} \equiv \frac{2}{3}\left(\vec{k}_{1}-\frac{1}{2}\left(\vec{k}_{2}+\vec{k}_{3}\right)\right)
\end{gathered}
$$

on top of the total momentum and obtains

$$
\begin{array}{r}
\rho_{p p n} \equiv \int \delta\left(\vec{P}^{\prime}-\vec{Q}\right) \delta\left(\sum_{i=1}^{3} \frac{k_{i}^{2}}{2 m_{N}}-Q_{0}-E_{3^{3} \mathrm{He}}\right) d \vec{k}_{1} d \vec{k}_{2} d \vec{k}_{3} d \vec{k}^{\prime} \\
=d \vec{k}^{\prime} \int d \vec{q} d \vec{p} \delta\left(\frac{p^{2}}{m_{N}}+\frac{3 q^{2}}{4 m_{N}}+\frac{\vec{Q}^{2}}{6 m_{N}}-Q_{0}-E_{3_{\mathrm{He}}}\right)=d \vec{k}^{\prime} \int d \hat{q} d \vec{p} \frac{2 m_{N}}{3}|\vec{q}|,
\end{array}
$$

with

$$
|\vec{q}|=\sqrt{\frac{4 m_{N}}{3}\left(Q_{0}+E_{3 \mathrm{He}}-\frac{\vec{Q}^{2}}{6 m_{N}}-\frac{p^{2}}{m_{N}}\right)},
$$

and the integration over $|\vec{p}|$ is between 0 and $p_{\max }$

$$
p_{\max }=\sqrt{m_{N}\left(Q_{0}+E_{3 \mathrm{He}}-\frac{\vec{Q}^{2}}{6 m_{N}}\right)} .
$$

Again the partial wave decomposition reveals (see [73]) that in the case when electron and ${ }^{3} \mathrm{He}$ are unpolarized only two response functions survive (this is, of course, known from standard symmetry arguments)

$$
\begin{gathered}
R_{L}=(2 \pi)^{6} \frac{1}{2} \sum_{m_{d} m_{p} m} \frac{2 m_{N}}{3}\left|\vec{q}_{0}\right| \int d \hat{q}\left|N_{0}^{p d}\right|^{2} \\
+(2 \pi)^{6} \frac{1}{2} \sum_{m_{1} m_{2} m_{3} m} \frac{2 m_{N}}{3} \int_{0}^{p_{\max }} d \vec{p} d \hat{q}|\vec{q}|\left|N_{0}^{p p n}\right|^{2} \\
R_{T}=(2 \pi)^{6} \frac{1}{2} \sum_{m_{d} m_{p} m} \frac{2 m_{N}}{3}\left|\vec{q}_{0}\right| \int d \hat{q}\left(\left|N_{1}^{p d}\right|^{2}+\left|N_{-1}^{p d}\right|^{2}\right) \\
+(2 \pi)^{6} \frac{1}{2} \sum_{m_{1} m_{2} m_{3} m} \frac{2 m_{N}}{3} \int_{0}^{p_{\max }} d \vec{p} d \hat{q}|\vec{q}|\left(\left|N_{1}^{p p n}\right|^{2}+\left|N_{-1}^{p p n}\right|^{2}\right) .
\end{gathered}
$$


Then the inclusive unpolarized scattering cross section reads

$$
\frac{d \sigma}{d \hat{k}^{\prime} d k_{0}^{\prime}}=\sigma_{M o t t}\left\{v_{L} R_{L}+v_{T} R_{T}\right\} .
$$

In (170) and (171) we added superscripts to the nuclear matrix elements according to the two types of final channels.

At this point it is adequate to describe another manner for evaluating the two response functions $R_{L}$ and $R_{T}[50,73]$. Both functions are of the type

$$
R=£ d f \delta\left(E_{f}-E_{i}-Q_{0}\right)\left|\left\langle\Psi_{f}|\mathcal{O}| \Psi_{i}\right\rangle\right|^{2}
$$

where $\mathcal{O}$ is an appropriate operator. Since the final states $\left|\Psi_{f}\right\rangle$ are eigenstates to the Hamiltonian $H$, one can use closure to evaluate $R$ as

$$
\begin{array}{r}
R=\sum d f\left\langle\Psi_{i}\right| \\
\mathcal{O}^{\dagger} \delta\left(H-E_{i}-Q_{0}\right)\left|\Psi_{f}\right\rangle\left\langle\Psi_{f}|\mathcal{O}| \Psi_{i}\right\rangle \\
=\left\langle\Psi_{i}\left|\mathcal{O}^{\dagger} \delta\left(H-E_{i}-Q_{0}\right)\right| \mathcal{O} \mid \Psi_{i}\right\rangle .
\end{array}
$$

The bound state does not contribute since $Q_{0}>0$. The result is easily converted into

$$
R=-\frac{1}{\pi} \Im\left\langle\Psi_{i}\left|\mathcal{O}^{\dagger} \frac{1}{Q_{0}+E_{i}-H+i \epsilon} \mathcal{O}\right| \Psi_{i}\right\rangle
$$

The remaining task is to evaluate the auxiliary state

$$
|\Phi\rangle \equiv \frac{1}{Q_{0}+E_{i}-H+i \epsilon} \mathcal{O}\left|\Psi_{i}\right\rangle
$$

which apparently fulfills the inhomogeneous Schrödinger equation

$$
(E+i \epsilon-H)|\Phi\rangle=\mathcal{O}\left|\Psi_{i}\right\rangle
$$

We introduced $E \equiv E_{i}+Q_{0}$. The Faddeev scheme is introduced by converting (177) into

$$
|\Phi\rangle=G_{0} \sum_{i=1}^{3}\left(V_{i}+V^{(i)}\right)|\Phi\rangle+G_{0} \mathcal{O}\left|\Psi_{i}\right\rangle .
$$

Similarly to the $3 \mathrm{NF}$, the operator $\mathcal{O}$ for three identical nucleons can always be split into three parts $\mathcal{O}_{i}$, symmetrical under the exchange of particles $j$ and $k$

$$
\mathcal{O}=\sum_{i=1}^{3} \mathcal{O}_{i}
$$


Therefore, the right hand side decomposes as

$$
|\Phi\rangle=G_{0} \sum_{i=1}^{3}\left|\Phi_{i}\right\rangle,
$$

where as before $\left|\Phi_{2}\right\rangle$ and $\left|\Phi_{3}\right\rangle$ result by cyclical and anticyclical permutations out of $\left|\Phi_{1}\right\rangle$. One obtains using the definition (10)

$$
\left(1-V_{1} G_{0}\right)\left|\Phi_{1}\right\rangle=V_{1} G_{0} P\left|\Phi_{1}\right\rangle+V^{(1)} G_{0}(1+P)\left|\Phi_{1}\right\rangle+\mathcal{O}_{1}\left|\Psi_{i}\right\rangle
$$

or

$$
\left|\Phi_{1}\right\rangle=\left(1+t G_{0}\right) \mathcal{O}_{1}\left|\Psi_{i}\right\rangle+\left(t_{1} G_{0} P+\left(1+t_{1} G_{0}\right) V^{(1)} G_{0}(1+P)\right)\left|\Phi_{1}\right\rangle .
$$

This is a Faddeev-like integral equation with the same kernel as in (65). The response function is then given as

$$
R=-\frac{3}{\pi} \Im\left\langle\Psi_{i}\left|\mathcal{O}_{1}^{\dagger}(1+P) G_{0}\right| \Phi_{1}\right\rangle
$$

The factor 3 arises since we kept only $\mathcal{O}_{1}^{\dagger}$.

The remaining cross section observables are for semi-exclusive and exclusive reactions on ${ }^{3} \mathrm{He}$. In the case of the processes ${ }^{3} \mathrm{He}\left(e, e^{\prime} p\right) d$ and ${ }^{3} \mathrm{He}\left(e, e^{\prime} N\right) N N$, where only one nucleon is measured in coincidence with the scattered electron, the plane spanned by the photon momentum and the detected nucleon momentum (hadronic plane) is in a general case rotated by an angle $\phi$ with respect to the plane spanned by the electron momenta (electronic plane). The dependence of the cross section on $\phi$ can be made explicit by introducing instead of the spherical unit vectors $\hat{e}_{ \pm 1}$ used up to now and which are perpendicular to $\hat{Q}$ (chosen in $\hat{z}$ direction) two other perpendicular unit vectors [102]

$$
\begin{gathered}
\hat{e}_{\perp} \equiv \widehat{\vec{Q} \times \vec{p}}=-\hat{x} \sin \phi+\hat{y} \cos \phi \\
\hat{e}_{\|} \equiv \hat{e}_{\perp} \widehat{\widehat{Q}} \vec{Q}=\hat{x} \cos \phi+\hat{y} \sin \phi .
\end{gathered}
$$

The unit vector $\hat{e}_{\perp}$ is perpendicular to the hadronic plane and $\hat{e}_{\|}$lies in that plane. The two pairs of unit vectors are connected by

$$
\hat{e}_{ \pm 1}=\mp \frac{e^{ \pm i \phi}}{\sqrt{2}}\left( \pm i \hat{e}_{\perp}+\hat{e}_{\|}\right)
$$

Introducing the components

$$
N_{\perp} \equiv \hat{e}_{\perp} \cdot \vec{N}
$$




$$
N_{\|} \equiv \hat{e}_{\|} \cdot \vec{N}
$$

of the nuclear matrix elements one finds the connections to the components used up to now

$$
N_{ \pm 1}=\mp \frac{e^{ \pm i \phi}}{\sqrt{2}}\left( \pm i N_{\perp}+N_{\|}\right) .
$$

The advantage of using $N_{\perp}$ and $N_{\|}$in the cross section formula is to make the $\phi$-dependence explicit.

After a simple algebra, using (189) and the definitions (148) of the $v$ 's, one obtains

$$
\begin{array}{r}
v_{L}\left|N_{0}\right|^{2}+v_{T}\left(\left|N_{1}\right|^{2}+\left|N_{-1}\right|^{2}\right)+ \\
v_{T T} 2 \Re\left(N_{1} N_{-1}^{*}\right)+v_{T L}(-2) \Re\left(N_{0}\left(N_{1}-N_{-1}\right)^{*}\right) \\
=v_{L}\left|N_{0}\right|^{2}+v_{T}\left(\left|N_{\perp}\right|^{2}+\left|N_{\|}\right|^{2}\right)+ \\
2 \sqrt{2} v_{T L} \cos \phi \Re\left(N_{0} N_{\|}^{*}\right)+v_{T T} \cos (2 \phi)\left(\left|N_{\perp}\right|^{2}-\left|N_{\|}\right|^{2}\right) .
\end{array}
$$

Thereby two terms proportional to $\Re\left(N_{0} N_{\perp}^{*}\right)$ and $\Re\left(N_{\perp} N_{\|}^{*}\right)$ have been dropped since they vanish here as is seen in a partial wave decomposition [48].

As is seen from (147) and (149) these sums generate the cross sections except for an overall factor. The rotational invariance around the $z$-axis (chosen in $\hat{Q}$ direction) guarantees that none of the quantities $N_{0}, N_{\|}$and $N_{\perp}$ depends on $\phi$.

It follows that the pd breakup cross section can be written in two forms

$$
\begin{array}{r}
\frac{d^{5} \sigma}{d \hat{k}^{\prime} d k_{0}^{\prime} d \hat{q}_{0}}=\sigma_{M o t t}\left[v_{L}\left|N_{0}\right|^{2}+v_{T}\left(\left|N_{1}\right|^{2}+\left|N_{-1}\right|^{2}\right)+\right. \\
\left.v_{T T} 2 \Re\left(N_{1} N_{-1}^{*}\right)+v_{T L}(-2) \Re\left(N_{0}\left(N_{1}-N_{-1}\right)^{*}\right)\right] \rho_{p d} \\
=\sigma_{M o t t}\left[v_{L}\left|N_{0}\right|^{2}+v_{T}\left(\left|N_{\perp}\right|^{2}+\left|N_{\|}\right|^{2}\right)+\right. \\
\left.2 \sqrt{2} v_{T L} \cos \phi \Re\left(N_{0} N_{\|}^{*}\right)+v_{T T} \cos (2 \phi)\left(\left|N_{\perp}\right|^{2}-\left|N_{\|}\right|^{2}\right)\right] \rho_{p d},
\end{array}
$$

where the second one shows the $\phi$ dependence explicitly.

In the case of the semi-exclusive ${ }^{3} \mathrm{He}\left(e, e^{\prime} p\right) p n$ or ${ }^{3} \mathrm{He}\left(e, e^{\prime} n\right) p p$ reactions one has to integrate over the internal (relative) momentum of the undetected pair of nucleons and one obtains for the cross section

$$
\begin{aligned}
\frac{d^{6} \sigma}{d \hat{k}^{\prime} d k_{0}^{\prime} d \vec{q}}= & \sigma_{M o t t} \mathcal{C} \frac{1}{2} m_{N} p \int d \hat{p}\left[v_{L}\left|N_{0}\right|^{2}+v_{T}\left(\left|N_{\perp}\right|^{2}+\left|N_{\|}\right|^{2}\right)+\right. \\
& \left.2 \sqrt{2} v_{T L} \cos \phi \Re\left(N_{0} N_{\|}^{*}\right)+v_{T T} \cos (2 \phi)\left(\left|N_{\perp}\right|^{2}-\left|N_{\|}\right|^{2}\right)\right]
\end{aligned}
$$


with

$$
p \equiv|\vec{p}|=\sqrt{m_{N}\left(Q_{0}+E_{3 \mathrm{He}}-\frac{\vec{Q}^{2}}{6 m_{N}}-\frac{3 \vec{q}^{2}}{4 m_{N}}\right)} .
$$

To avoid double-counting the $\mathcal{C}$ factor is equal $1 / 2$ in case when the two undetected nucleons are identical (protons). Otherwise $\mathcal{C}=1$.

Lastly there is the complete breakup of ${ }^{3} \mathrm{He}$ measured exclusively by detecting two nucleons in coincidence with the scattered electron. This leads to an eightfold differential cross section. The phase space factor is

$$
\begin{array}{r}
\rho=\int \delta\left(\vec{P}^{\prime}-\vec{Q}\right) \delta\left(\sum_{i=1}^{3} \frac{k_{i}^{2}}{2 m_{N}}-Q_{0}-E_{3 \mathrm{He}}\right) d \vec{k}_{1} d \vec{k}_{2} d \vec{k}_{3} d \vec{k}^{\prime} \\
=d \vec{k}^{\prime} d \hat{k}_{1} d \hat{k}_{2} d E_{1} \frac{m^{2}\left|\vec{k}_{1}\right|\left|\vec{k}_{2}\right|}{\left|1-\frac{\vec{k}_{2} \cdot \vec{k}_{3}}{k_{2}^{2}}\right|}
\end{array}
$$

where $\vec{k}_{3}=\vec{Q}-\vec{k}_{1}-\vec{k}_{2}$ and $\left|\vec{k}_{2}\right|$ is determined kinematically from the given energy of the first nucleon $E_{1}$ and the directions of the first $\left(\hat{k}_{1}\right)$ and the second $\left(\hat{k}_{2}\right)$ nucleons detected in coincidence. At some values of the momenta the denominator in (194) can vanish. One avoids that singularity by representing the breakup cross section along the kinematically allowed locus in the $E_{1}-E_{2}$ plane and parameterizing it by the arc-length $S$ along that locus. This is a well known and usual device for the treatment of the Nd breakup process [103]. It leads to

$$
\rho=d \vec{k}^{\prime} d \hat{k}_{1} d \hat{k}_{2} d S \frac{m^{2}\left|\vec{k}_{1}\right|\left|\vec{k}_{2}\right|}{\sqrt{\left(1-\frac{\vec{k}_{2} \cdot \vec{k}_{3}}{k_{2}^{2}}\right)^{2}+\left(1-\frac{\vec{k}_{1} \cdot \vec{k}_{3}}{k_{1}^{2}}\right)^{2}}}
$$

and one obtains the following form for the complete breakup cross section expressed in terms of four response functions

$$
\frac{d^{8} \sigma}{d \hat{k}^{\prime} d k_{0}^{\prime} d \hat{k}_{1} d \hat{k}_{2} d S}=\sigma_{M o t t}\left[v_{L} R_{L}+v_{T} R_{T}+v_{T T} R_{T T}+v_{T L} R_{T L}\right] \rho .
$$

One can also relate the nuclear matrix elements to the unit vectors $\hat{e}_{\perp}$ and $\hat{e}_{\|}$which leads to six structure functions [49].

The inclusion of polarizations opens a wide field. We only mention cases which have already been studied experimentally in the $3 \mathrm{~N}$ system or which are on the list of our predictions (see section VII). In inclusive scattering, when only the outgoing electron is detected, two more response functions beyond the ones in (172) occur in the cross section. They 


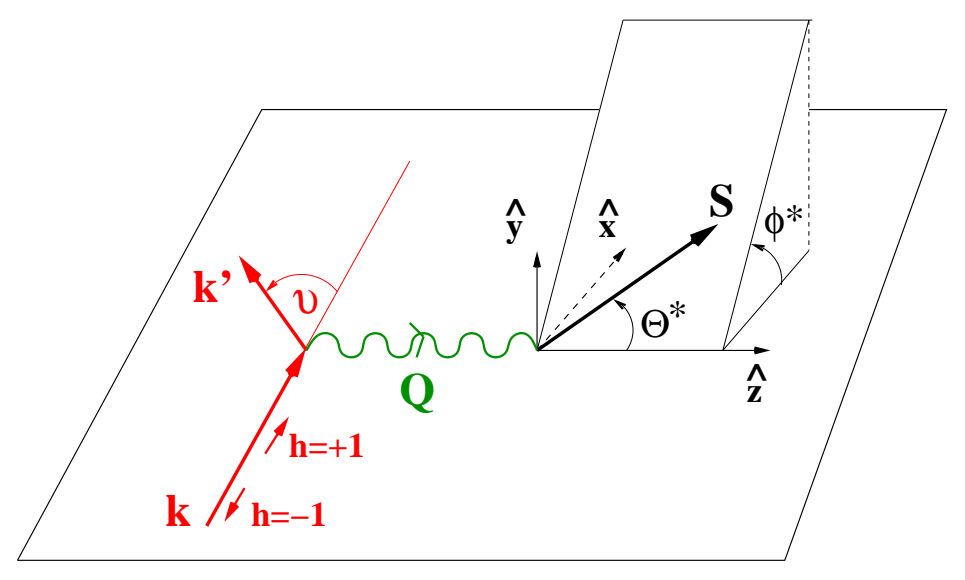

FIG. 6: Definition of the two angles $\left(\theta^{\star}, \phi^{\star}\right)$ used to specify the initial ${ }^{3} \mathrm{He}$ polarization.

go with the helicity $h$ of the initial electron state as given in (147) (see [99]). This leads naturally to an asymmetry defined by

$$
A \equiv \frac{\sigma(h=+1)-\sigma(h=-1)}{\sigma(h=+1)+\sigma(h=-1)}=\frac{v_{T^{\prime}} R_{T^{\prime}}+v_{T L^{\prime}} R_{T L^{\prime}}}{v_{L} R_{L}+v_{T} R_{T}} .
$$

This quantity has been investigated especially for the case of additionally polarized ${ }^{3} \mathrm{He}$. If the quantization axis of ${ }^{3} \mathrm{He}$ points in the direction given by the two polar angles $\left(\theta^{\star}, \phi^{\star}\right)$ (see Fig. 6), the ${ }^{3}$ He state can be written as

$$
\left|\Psi_{3_{\mathrm{He}}} m\right\rangle_{\left(\theta^{\star}, \phi^{\star}\right)}=\sum_{m^{\prime}}\left|\Psi_{{ }^{\mathrm{He}}} m^{\prime}\right\rangle D_{m^{\prime} m}^{\left(\frac{1}{2}\right)}\left(\phi^{\star}, \theta^{\star}, 0\right),
$$

where $\left|\Psi_{3 \mathrm{He}} m^{\prime}\right\rangle$ is quantized in $z$-direction. We refer to [73] where the dependence on $\left(\theta^{\star}, \phi^{\star}\right)$ of the two new response functions has been worked out. There also the generalization necessary for evaluating the new response functions with the help of the closure relation is detailed. One obtains the explicit $\left(\theta^{\star}, \phi^{\star}\right)$ dependence as [99]

$$
\begin{gathered}
R_{T^{\prime}} \equiv-\tilde{R}_{T^{\prime}} \cos \theta^{\star} \\
R_{T L^{\prime}} \equiv-2 \tilde{R}_{T L^{\prime}} \sin \theta^{\star} \cos \phi^{\star} .
\end{gathered}
$$

Consequently the asymmetry reads now

$$
A=-\frac{v_{T^{\prime}} \tilde{R}_{T^{\prime}} \cos \theta^{\star}+2 v_{T L^{\prime}} \tilde{R}_{T L^{\prime}} \sin \theta^{\star} \cos \phi^{\star}}{v_{L} R_{L}+v_{T} R_{T}} .
$$

Polarizing the ${ }^{3}$ He target $\operatorname{spin}(m=1 / 2)$ along the virtual photon direction $\hat{Q}\left(\theta^{\star}=0^{\circ}\right)$ one selects the transverse asymmetry $A_{T^{\prime}}$ (proportional to $\tilde{R}_{T^{\prime}}$ ), whereas taking $\theta^{\star}=90^{\circ}$ one gets the transverse-longitudinal asymmetry $A_{T L^{\prime}}$ (proportional to $\tilde{R}_{T L^{\prime}}$ ). 
In the case of the semiexclusive ${ }^{3} \mathrm{He}\left(\vec{e}, e^{\prime} p\right) p n$ and ${ }^{3} \mathrm{He}\left(\vec{e}, e^{\prime} n\right) p p$ processes, the asymmetry is defined analogously to (197). However, an additional integration over the direction of the relative momentum of the two undetected nucleons is needed and, according to (147), two additional response functions, $R_{T T}$ and $R_{T L}$, occur in the denominator. One obtains

$$
A \equiv \frac{\int d \hat{p}\left(v_{T^{\prime}} R_{T^{\prime}}+v_{T L^{\prime}} R_{T L^{\prime}}\right)}{\int d \hat{p}\left(v_{L} R_{L}+v_{T} R_{T}+v_{T T} R_{T T}+v_{T L} R_{T L}\right)},
$$

and one can define again the asymmetries $A_{\perp}$ and $A_{\|}$corresponding to two different initial ${ }^{3} \mathrm{He}$ spin orientations with respect to the photon direction.

In the case of the pd breakup process ${ }^{3} \mathrm{He}\left(\vec{e}, e^{\prime} p\right) d$ the asymmetries are defined correspondingly.

The more intricate processes $\overrightarrow{{ }^{3} \mathrm{He}}\left(\vec{e}, e^{\prime} \vec{p}\right) d$ and $\overrightarrow{{ }^{3} \mathrm{He}}\left(\vec{e}, e^{\prime} \vec{d}\right) p$, where also the polarization of the final particles is measured, are dealt with in section VII.

Spin asymmetries in the exclusive $3 \mathrm{~N}$ breakup reaction have not yet been measured or investigated theoretically in the $3 \mathrm{~N}$ system to the best of our knowledge but the formal extensions are straightforward.

The last group of observables we want to address in this review are photodisintegration of ${ }^{3} \mathrm{He}$ and Nd capture processes. Because of lack of own experience we shall, however, not discuss Compton scattering on ${ }^{3} \mathrm{He}$ nor Bremsstrahlung in the $3 \mathrm{~N}$ system.

The $S$-matrix element for photodisintegration into the final channel $f$, which is either pd or ppn fragmentation, is given as

$$
S_{f i}=-i(2 \pi)^{4} \delta\left(P^{\prime}-P-Q\right) \frac{1}{\sqrt{2|\vec{Q}|}} \frac{1}{(2 \pi)^{3 / 2}}\left\langle\Psi_{f}^{(-)} \vec{P}^{\prime}\left|\vec{\epsilon}_{\lambda} \cdot \vec{j}(0)\right| \Psi \vec{P}\right\rangle,
$$

with $\left\langle\Psi_{f}^{(-)}\right|$the $3 \mathrm{~N}$ scattering state with appropriate asymptotic quantum numbers $f$. Further $\vec{\epsilon}_{\lambda}$ is the polarization vector for the initial photon whose momentum defines the z-direction. It results in the differential cross section

$$
d \sigma=(2 \pi)^{4} \frac{1}{2|\vec{Q}|} \delta\left(P^{\prime}-P-Q\right) d f\left|\left\langle\Psi_{f}^{(-)} \vec{P}^{\prime}\left|\vec{\epsilon}_{\lambda} \cdot \vec{j}(0)\right| \Psi \vec{P}\right\rangle\right|^{2} .
$$

Neglecting any polarization the differential cross section for pd fragmentation in the lab system is

$$
\frac{d \sigma}{d \hat{k}_{p}}=(2 \pi)^{4} \alpha \frac{1}{2|\vec{Q}|} \frac{k_{p}^{2}}{\mid \frac{k_{p}}{m_{N}}-\frac{\vec{k}_{d} \cdot \vec{k}_{p}}{2 m_{N}\left|\vec{k}_{p}\right|}} \frac{1}{2} \sum_{m m_{N} m_{d}}\left(\left|N_{1}\right|^{2}+\left|N_{-1}\right|^{2}\right) .
$$


In the nuclear matrix elements

$$
N_{ \pm 1} \equiv\left\langle\Psi_{\vec{q}}^{(-)}\left|\vec{j}_{ \pm 1}(0)\right| \Psi\right\rangle
$$

the final $3 \mathrm{~N}$ scattering state depends on the asymptotic Jacobi momentum $\vec{q}$ expressed in terms of the final lab momenta $\vec{k}_{p}$ and $\vec{k}_{d}$ as

$$
\vec{q} \equiv \frac{2}{3}\left(\vec{k}_{p}-\frac{1}{2} \vec{k}_{d}\right) .
$$

In the case of the $3 \mathrm{~N}$ breakup the unpolarized cross section is

$$
\frac{d^{5} \sigma}{d \hat{k}_{1} d \hat{k}_{2} d S}=\frac{2 \pi^{2} \alpha}{E_{\gamma}} \frac{1}{2} \sum_{m m_{1} m_{2} m_{3}}\left(\left|N_{1}\right|^{2}+\left|N_{-1}\right|^{2}\right) \frac{m_{N}^{2}\left|\vec{k}_{1}\right|\left|\vec{k}_{2}\right|}{\sqrt{\left(1-\frac{\vec{k}_{2} \cdot \vec{k}_{3}}{k_{2}^{2}}\right)^{2}+\left(1-\frac{\vec{k}_{1} \cdot \vec{k}_{3}}{k_{1}^{2}}\right)^{2}}} .
$$

Now the nuclear matrix element depends of course on the asymptotic Jacobi momenta $\vec{p}$ and $\vec{q}$ related to the lab momenta as given before in (20) and (21).

The cross sections for the semi-exclusive processes ${ }^{3} \mathrm{He}(\gamma, p)$ and ${ }^{3} \mathrm{He}(\gamma, n)$ in the $3 \mathrm{~N}$ breakup are

$$
\frac{d^{3} \sigma}{d \hat{k}_{1} d E_{1}}=\frac{2 \pi^{2} \alpha}{E_{\gamma}} m_{N}^{2} \frac{1}{2}\left|\vec{k}_{1}\right||\vec{p}| \mathcal{C} \int d \hat{p} \frac{1}{2} \sum_{m m_{1} m_{2} m_{3}}\left(\left|N_{1}\right|^{2}+\left|N_{-1}\right|^{2}\right),
$$

where $|\vec{p}|$ and $\hat{p}$ are the magnitude and direction of the relative momentum between the undetected nucleons 2 and 3 and $\mathcal{C}$ as given before.

The availability of high intensity polarized $\gamma$ sources [104] made it possible to measure semiexclusive reactions with a linearly polarized incoming $\gamma$ beam and even with a polarized ${ }^{3} \mathrm{He}$ target. Due to the polarization of the incoming $\gamma$ 's and/or of the ${ }^{3} \mathrm{He}$ target, the energy spectrum of the outgoing nucleon taken at a particular polar lab angle $\theta$ depends on the azimuthal angle $\phi$, leading to an asymmetry of the measured cross sections. Assuming that the incoming $\gamma$ 's are linearly polarized along the x-axis with a nonzero component $P_{0}^{\gamma}$ and that the ${ }^{3} \mathrm{He}$ target nucleus is polarized along the y-axis with polarization $P_{0}^{3} \mathrm{He}$, one obtains for the cross section measured with a nucleon detector placed at angles $(\theta, \phi)$ :

$$
\begin{aligned}
\sigma_{\gamma,{ }^{3} \mathrm{He}}^{\text {pol }}= & \sigma_{\gamma,{ }^{3} \mathrm{He} e}^{\text {unpol }}\left[1+P_{0}^{\gamma} \cos (2 \phi) A_{x}^{\gamma}+P_{0}^{3} \mathrm{He} \cos (\phi) A_{y}^{3} \mathrm{He}+\right. \\
& \left.P_{0}^{\gamma} \cos (2 \phi) P_{0}^{{ }^{3} \mathrm{He}} \cos (\phi) C_{x, y}^{\gamma,{ }^{3} \mathrm{He}}+P_{0}^{\gamma} \sin (2 \phi) P_{0}^{3} \mathrm{He} \sin (\phi) C_{y, x}^{\gamma,{ }^{3} \mathrm{He}}\right] .
\end{aligned}
$$

The analyzing powers $A_{x}^{\gamma}(\theta), A_{y}^{3} \mathrm{He}(\theta)$ and spin correlation coefficients $C_{x, y(y, x)}^{\gamma,{ }^{3} \mathrm{He}}(\theta)$ are expressed through the nuclear matrix element $N_{m_{1} m_{2} m_{3}, \lambda m} \equiv N_{m_{i}, \lambda m}$ by:

$$
A_{x}^{\gamma}(\theta) \equiv \frac{\sum_{m_{i} m}\left(2 \Re\left\{N_{m_{i},-1 m} N_{m_{i},+1 m}^{*}\right\}\right)}{\sum_{m_{i} m}\left(\left|N_{m_{i},+1 m}\right|^{2}+\left|N_{m_{i},-1 m}\right|^{2}\right)}
$$




$$
\begin{aligned}
A_{y}^{3} \mathrm{He}(\theta) & \equiv \frac{\sum_{m_{i}}\left(-2 \Im\left\{N_{m_{i},-1-\frac{1}{2}} N_{m_{i},-1 \frac{1}{2}}^{*}\right\}-2 \Im\left\{N_{m_{i},+1-\frac{1}{2}} N_{m_{i},+1 \frac{1}{2}}^{*}\right\}\right)}{\sum_{m_{i} m}\left(\left|N_{m_{i},+1 m}\right|^{2}+\left|N_{m_{i},-1 m}\right|^{2}\right)} \\
C_{x, y}^{\gamma,{ }^{3} \mathrm{He}}(\theta) & \equiv \frac{\sum_{m_{i}}\left(-2 \Im\left\{N_{m_{i},-1-\frac{1}{2}} N_{m_{i},+1 \frac{1}{2}}^{*}\right\}+2 \Im\left\{N_{m_{i},-1 \frac{1}{2}} N_{m_{i},+1-\frac{1}{2}}^{*}\right\}\right)}{\sum_{m_{i} m}\left(\left|N_{m_{i},+1 m}\right|^{2}+\left|N_{m_{i},-1 m}\right|^{2}\right)} \\
C_{y, x}^{\gamma,{ }^{3} \mathrm{He}}(\theta) & \equiv \frac{\sum_{m_{i}}\left(2 \Im\left\{N_{m_{i},-1-\frac{1}{2}} N_{m_{i},+1 \frac{1}{2}}^{*}\right\}+2 \Im\left\{N_{m_{i},-1 \frac{1}{2}} N_{m_{i},+1-\frac{1}{2}}^{*}\right\}\right)}{\sum_{m_{i} m}\left(\left|N_{m_{i},+1 m}\right|^{2}+\left|N_{m_{i},-1 m}\right|^{2}\right)},
\end{aligned}
$$

where $m$ is the spin projection of the ${ }^{3} \mathrm{He}$ target and $m_{i}$ are the spin projections of the outgoing nucleons.

Finally we come to the Nd capture process. The angular distribution of the photon in the system of total momentum zero neglecting any polarization is

$$
\frac{d \sigma}{d \hat{Q}}=(2 \pi)^{2} \alpha \frac{1}{6} \sum_{m m_{N} m_{d}}\left(\left|N_{1}\right|^{2}+\left|N_{-1}\right|^{2}\right) \frac{2 m_{N} Q}{3\left|\vec{q}_{0}\right|},
$$

where $\vec{q}_{0}$ is the relative nucleon-deuteron momentum in the initial state, which also defines the $z$-direction.

For this reaction vector and tensor analyzing powers have been measured. This comprises the cases that the initial proton is polarized perpendicular to the scattering plane or the initial deuteron is vector or tensor polarized. Now a more detailed notation for the nuclear matrix element is needed, namely

$$
N_{\lambda m, m_{N} m_{d}} \equiv\left\langle\Psi_{3_{\mathrm{He}}} m \vec{Q}\left|\vec{\epsilon}_{\lambda} \cdot \vec{j}(0)\right| \Psi_{\vec{q}_{0}} m_{N} m_{d}\right\rangle
$$

showing explicitly the dependence on the spin magnetic quantum numbers $m, m_{N}, m_{d}$ and $\lambda$ of ${ }^{3} \mathrm{He}$, the nucleon, the deuteron and the photon, respectively. Then according to the standard formalism [18] one obtains the nucleon analyzing power $A_{y}$ as

$$
A_{y}=i \sqrt{2} \frac{\sum_{m_{N}, m_{N}^{\prime}, m_{d}, \lambda, m} \sqrt{2}(-1)^{\frac{1}{2}-m_{N}} C\left(\frac{1}{2}, \frac{1}{2}, 1 ; m_{N}^{\prime},-m_{N}, 1\right) N_{\lambda, m, m_{N}, m_{d}} N_{\lambda, m, m_{N}^{\prime}, m_{d}}^{\star}}{\sum_{m_{N}, m_{d}, m}\left(\left|N_{+1}\right|^{2}+\left|N_{-1}\right|^{2}\right)},
$$

the deuteron vector analyzing power $i T_{11}$

$$
i T_{11}=i \frac{\sum_{m_{N}, m_{d}, m_{d}^{\prime}, \lambda, m} \sqrt{3}(-1)^{1-m_{d}} C\left(1,1,1 ; m_{d}^{\prime},-m_{d}, 1\right) N_{\lambda, m, m_{N}, m_{d}} N_{\lambda, m, m_{N}, m_{d}^{\prime}}^{\star}}{\sum_{m_{N}, m_{d}, m}\left(\left|N_{+1}\right|^{2}+\left|N_{-1}\right|^{2}\right)},
$$

and the deuteron tensor analyzing powers $T_{j k}$

$$
T_{j k}=\frac{\sum_{m_{N}, m_{d}, m_{d}^{\prime}, \lambda, m} \sqrt{3}(-1)^{1-m_{d}} C\left(1,1, j ; m_{d}^{\prime},-m_{d}, k\right) N_{\lambda, m, m_{N}, m_{d}} N_{\lambda, m, m_{N}, m_{d}^{\prime}}^{\star}}{\sum_{m_{N}, m_{d}, m}\left(\left|N_{+1}\right|^{2}+\left|N_{-1}\right|^{2}\right)} .
$$


Similarly to the unpolarized cross section these observables can be parametrized by e.g. the c.m. scattering angle of the outgoing photon against the direction of the incoming deuteron.

\section{THE PERFORMANCE}

This is the most central but also likely less pleasant part of this review. Here we would like to indicate the way we evaluate the nuclear matrix elements, which requires the solution of various types of Faddeev-like equations.

Let us regard the electron induced pd breakup matrix element (58), where the state $|\tilde{U}\rangle$ obeys the Faddeev like equation (44). Neglecting all rescattering $|\tilde{U}\rangle$ reduces to the driving term in (44). This leads to the nuclear matrix element in the symmetrized plane wave approximation, denoted as PWIAS,

$$
N^{p d, \text { PWIAS }}=\frac{1}{2}\left\langle\phi_{q^{\prime}}|P(1+P) \mathcal{O}| \Psi\right\rangle=\left\langle\phi_{q^{\prime}}|(1+P) \mathcal{O}| \Psi\right\rangle .
$$

The second equality is due to the identity (40). Now we have to insert explicit choices for the operator $\mathcal{O}$. In the case of $N_{0}$ the density operator appears while $N_{ \pm 1}$ is driven by the transversal pieces of the vector current. We start with the single nucleon contributions for the density and the vector current. Since $\mathcal{O}$ is fully symmetrical and $\left\langle\phi_{q^{\prime}}\right|(1+P)$ as well as $|\Psi\rangle$ are fully antisymmetrical, it is sufficient to choose the operators acting on one nucleon, say nucleon 1, and multiply that matrix element by the factor 3 . Thus still not specifying the component of $j_{S N}^{\mu}$, one has in the very first step

$$
N^{p d, \text { PWIAS }} \equiv 3\left\langle\phi_{q^{\prime}}\left|(1+P) j_{S N}(1)\right| \Psi\right\rangle
$$

The nonrelativistic $3 \mathrm{~N}$ states are conventionally expressed in terms of Jacobi momenta $\vec{p}$ and $\vec{q}$ as defined in (20) and (21) for one choice of the two-nucleon subsystem. Thus inserting completeness relations one obtains

$$
N^{p d, \text { PWIAS }}=3 \int\left\langle\phi_{q^{\prime}}|(1+P)| \vec{p}^{\prime} \vec{q}^{\prime}\right\rangle\left\langle\vec{p}^{\prime} \vec{q}^{\prime}\left|j_{S N}(1)\right| \vec{p} \vec{q}\right\rangle\langle\vec{p} \vec{q} \mid \Psi\rangle
$$

where of course integration over $\vec{p}, \vec{q}, \vec{p}^{\prime}, \vec{q}^{\prime}$ is assumed. The free states $|\vec{p} \vec{q}\rangle$ also include spin and isospin magnetic quantum numbers for the three nucleons but for the sake of a simpler notation we dropped that information and the accompanying discrete sums. Now

because of the overall $\delta$-function $\delta\left(\vec{P}^{\prime}-\vec{P}-\vec{Q}\right)$ which is taken care of in evaluating the 
observables, the matrix element of the single particle operator in the space of the $3 \mathrm{~N}$ Jacobi momenta is

$$
\left\langle\vec{p}^{\prime} \vec{q}^{\prime}\left|j_{S N}(1)\right| \vec{p} \vec{q}\right\rangle=J(\vec{Q}, \vec{q}) \delta\left(\vec{p}^{\prime}-\vec{p}\right) \delta\left(\vec{q}^{\prime}-\vec{q}-\frac{2}{3} \vec{Q}\right),
$$

and it results in

$$
N^{p d, \text { PWIAS }}=3 \int\left\langle\phi_{q^{\prime}}|(1+P)| \vec{p} \vec{q}\right\rangle J(\vec{Q}, \vec{q})\left\langle\vec{p} \vec{q}-\frac{2}{3} \vec{Q} \mid \Psi\right\rangle .
$$

Three components of $j$ occur in the response functions, $j^{0}$ and $j_{ \pm 1}$. According to (78) the function $J^{0}(\vec{Q}, \vec{q})$ related to the density operator is in lowest order

$$
J^{0}(\vec{Q}, \vec{q})=G_{E}^{p}(\vec{Q}) \Pi^{p}+G_{E}^{n}(\vec{Q}) \Pi^{n}
$$

and according to (72) the functions related to the spherical components of the spin current are

$$
J_{ \pm 1}^{\text {spin }}(\vec{Q}, \vec{q})=\frac{i}{2 m}\left(G_{M}^{p}(\vec{Q}) \Pi^{p}+G_{M}^{n}(\vec{Q}) \Pi^{n}\right)(\vec{\sigma}(1) \times \vec{Q})_{ \pm 1} .
$$

In the case of the convection current (see (72) ) one expresses the individual nucleon momenta by the Jacobi momentum $\vec{q}$

$$
\frac{\vec{k}_{1}+\vec{k}_{1}^{\prime}}{2 m_{N}}=\frac{\frac{2}{3} \vec{P}+\vec{Q}+2 \vec{q}}{2 m_{N}} .
$$

Then in the lab system and choosing the $\hat{z}$ and $\hat{Q}$ directions to coincide only $\vec{q}$ survives for the spherical components and the corresponding functions for the convection current are

$$
J_{ \pm 1}^{\text {convect }}(\vec{Q}, \vec{q})=\frac{q_{ \pm 1}}{m}\left(G_{E}^{p}(\vec{Q}) \Pi^{p}+G_{E}^{n}(\vec{Q}) \Pi^{n}\right)
$$

The bra state in (217)-(219) and (221) composed of a deuteron and a state of free relative motion of nucleon 1 and the deuteron is

$$
\left\langle\phi_{\vec{q}^{\prime}} \mid \vec{p} \vec{q}\right\rangle=\left\langle\varphi_{d} \mid \vec{p}\right\rangle \delta\left(\vec{q}^{\prime}-\vec{q}\right)
$$

The permutations $P$ are most conveniently evaluated as described in [69] (here we drop the spin-isospin parts for simplicity)

$$
P|\vec{p} \vec{q}\rangle_{1}=|\vec{p} \vec{q}\rangle_{2}+|\vec{p} \vec{q}\rangle_{3}=\left|-\frac{1}{2} \vec{p}-\frac{3}{4} \vec{q}, \vec{p}-\frac{1}{2} \vec{q}\right\rangle_{1}+\left|-\frac{1}{2} \vec{p}+\frac{3}{4} \vec{q},-\vec{p}-\frac{1}{2} \vec{q}\right\rangle_{1} .
$$

In (227) we added subscripts. The subscript " 1 " indicates that $\vec{p}$ refers to the subsystem (23) and $\vec{q}$ is the relative momentum of particle 1 in relation to the pair (23). This choice appears in (221). Now $|\vec{p} \vec{q}\rangle_{2}\left(|\vec{p} \vec{q}\rangle_{3}\right)$ signifies that the momenta did not change but they refer to 
different two-body subsystems. The particles are cyclically (anticyclically) permuted, thus "2" points to the subsystem (31) and "3" to the subsystem (12). In the second equality the Jacobi momenta of the type "2" and "3" are re-expressed in terms of linear combinations of the Jacobi momenta of the type "1". Therefore, one can again use (226) to evaluate $\left\langle\phi_{\vec{q}^{\prime}}|P| \vec{p} \vec{q}\right\rangle$.

The second part of $|\tilde{U}\rangle$ in (44) depends on the solution $|\tilde{U}\rangle$ of that equation. In the future it might be advisable to solve that equation directly in vector variables. First steps in that direction have been already undertaken [105]. We still work using partial wave decomposition and would like to indicate some formal structures. A complete set of basis states for three nucleons is

$$
|p q \alpha\rangle \equiv\left|p q(l s) j\left(\lambda \frac{1}{2}\right) I(j I) J m ;\left(t \frac{1}{2}\right) T m_{T}\right\rangle,
$$

where $p$ and $q$ are the magnitudes of Jacobi momenta, $l, s$, and $j$ orbital, spin, and total angular momentum quantum numbers of the two-body subsystem, $\lambda, \frac{1}{2}$, and $I$ orbital, spin, and total angular momentum quantum numbers of the third particle. Then $j$ and $I$ are coupled to the total $3 \mathrm{~N}$ angular momentum $J$. Finally the two-body subsystem isospin $t$ is coupled with the one of the third particle to the total isospin $T$.

Because of the identity of the nucleons not all quantum numbers are allowed and one has the condition

$$
(-1)^{l+s+t}=-1
$$

That set of basis states is complete

$$
\sum_{\alpha} \int d p p^{2} \int d q q^{2}|p q \alpha\rangle\langle p q \alpha|=1
$$

An equation like (44) is now projected onto those states

$$
\langle p q \alpha \mid \tilde{U}\rangle=\left\langle p q \alpha \mid \tilde{U}^{0}\right\rangle+\left\langle p q \alpha\left|t G_{0} P+\ldots\right| \tilde{U}\right\rangle
$$

We abbreviated the driving term by $\left|\tilde{U}^{0}\right\rangle$ and the dots stand for the second part of the integral kernel. Now $t G_{0} P$ is exactly the kernel which occurs in our standard Faddeev like integral equation for $3 \mathrm{~N}$ scattering $[16,18,71]$. In $[18,71]$ that partial wave decomposition has been displayed in all detail, namely the evaluation of the permutation operator in the chosen basis $\mid p q \alpha>$, the solution of the Lippmann-Schwinger two-body equation leading to the representation of $t$ in that basis, and the treatment of the logarithmic singularities 
arising from the free propagator $G_{0}$. Therefore, we shall not repeat all that here. Clearly one ends up with a set of coupled integral equations in two variables for each total angular momentum $J$, total isospin $T$, and parity $\pi=(-1)^{l+\lambda}$. The second part of the kernel, which involves the three nucleon force $V^{(1)}$ in interference with the NN $t$-operator, appears to be more complex but in fact it is easier for numerical treatment. We refer for its representation to $[106]$.

Let us now come back to the driving term and regard its projection on the basis states (228) in the case of a single nucleon operator $\mathcal{O}$ :

$$
\begin{array}{r}
\langle p q \alpha|(1+P) \mathcal{O}| \Psi\rangle=3\left\langle p q \alpha\left|(1+P) j_{S N}(1)\right| \Psi\right\rangle= \\
3\left\langle p q \alpha\left|j_{S N}(1)\right| \Psi\right\rangle+3\left\langle p q \alpha\left|P j_{S N}(1)\right| \Psi\right\rangle
\end{array}
$$

We could extract again the factor 3 since $\langle p q \alpha|(1+P)$ is fully antisymmetrical due to the condition (229). As an example we now show for the simplest case of the density operator the partial wave decomposition of $\left\langle p q \alpha\left|j_{S N}^{0}(1)\right| \Psi\right\rangle$. Comparing (220) and (221) we see that

$$
\left\langle\vec{p} \vec{q}\left|j_{S N}^{0}(1)\right| \Psi\right\rangle=J^{0}(\vec{Q})\left\langle\vec{p}, \vec{q}-\frac{2}{3} \vec{Q} \mid \Psi\right\rangle
$$

Consequently

$$
\begin{aligned}
& \left\langle p q \alpha\left|j_{S N}^{0}(1)\right| \Psi\right\rangle=J^{0}(\vec{Q}) \int\left\langle p q \alpha \mid \vec{p}^{\prime} \vec{q}^{\prime}\right\rangle\left\langle\vec{p}^{\prime} \vec{q}^{\prime}-\frac{2}{3} \vec{Q} \mid \Psi\right\rangle \\
= & J^{0}(\vec{Q}) \int\left\langle p q \alpha \mid \vec{p}^{\prime} \vec{q}^{\prime}\right\rangle \sum\left\langle\vec{p}^{\prime} \vec{q}^{\prime}-\frac{2}{3} \vec{Q} \mid p^{\prime \prime} q^{\prime \prime} \alpha^{\prime \prime}\right\rangle \Psi_{\alpha^{\prime \prime}}\left(p^{\prime \prime}, q^{\prime \prime}\right) .
\end{aligned}
$$

In the second equality we inserted the partial wave decomposition of the $3 \mathrm{~N}$ bound state. The wave function components $\Psi_{\alpha^{\prime \prime}}\left(p^{\prime \prime}, q^{\prime \prime}\right) \equiv\left\langle p^{\prime \prime} q^{\prime \prime} \alpha^{\prime \prime} \mid \Psi\right\rangle$ result from solving the 3N bound state Faddeev equation [15]. The rather tedious but known steps to evaluate the overlaps between momentum vector states with shifted vector arguments and our partial wave projected basis states as well as the six fold integration can be carried through analytically with the techniques presented in [69]. Results for various partial wave projected matrix elements can be found in $[51,73,101,107]$. As an example the expression in (234) results for an arbitrary direction of $\vec{Q}$ in

$$
\begin{array}{r}
\left\langle p q(l s) j\left(\lambda \frac{1}{2}\right) I(j I) J m ;\left(t \frac{1}{2}\right) T m_{T}\left|j_{S N}^{0}(1)\right| \Psi m^{\prime \prime}\right\rangle= \\
I\left(t, T, M_{T}\right) \sqrt{\pi}(-1)^{j} \sqrt{\hat{J}} \sqrt{\hat{I}}
\end{array}
$$




$$
\begin{array}{r}
\sum_{\alpha^{\prime \prime}} \delta_{l^{\prime \prime} l} \delta_{s^{\prime \prime} s} \delta_{j^{\prime \prime} j} \delta_{t^{\prime \prime} t} \sqrt{\hat{\lambda^{\prime \prime}}} \sqrt{\hat{I^{\prime \prime}}} \sqrt{\left(2 \lambda^{\prime \prime}+1\right) !}(-1)^{\lambda^{\prime \prime}} \\
\sum_{\lambda_{1}^{\prime \prime}+\lambda_{2}^{\prime \prime}=\lambda^{\prime \prime}}(q)^{\lambda_{1}^{\prime \prime}}\left(\frac{2}{3} Q\right)^{\lambda_{2}^{\prime \prime}}(-1)^{\lambda_{2}^{\prime \prime}} \frac{1}{\sqrt{\left(2 \lambda_{1}^{\prime \prime}\right) !\left(2 \lambda_{2}^{\prime \prime}\right) !}} \\
\sum_{g}\left(k \lambda_{2}^{\prime \prime} g ; 000\right)\left\{\begin{array}{ccc}
g & \lambda & \lambda^{\prime \prime} \\
\lambda_{1}^{\prime \prime} & \lambda_{2}^{\prime \prime} & k
\end{array}\right\}\left\{\begin{array}{ccc}
I & \lambda & \frac{1}{2} \\
\lambda^{\prime \prime} & I^{\prime \prime} & g
\end{array}\right\}\left\{\begin{array}{lll}
g & I & I^{\prime \prime} \\
j & \frac{1}{2} & J
\end{array}\right\} \\
\left(J g \frac{1}{2} ; m, m^{\prime \prime}-m, m^{\prime \prime}\right) Y_{g, m^{\prime \prime}-m}(\hat{Q}),
\end{array}
$$

where

$$
g_{k} \equiv g_{k}\left(p, q,|\vec{Q}| ; \alpha^{\prime \prime}\right)=\int_{-1}^{1} d x P_{k}(x) \frac{\Psi_{\alpha^{\prime \prime}}(p, \tilde{q})}{\tilde{q}^{\lambda^{\prime \prime}}}
$$

with

$$
\tilde{q}=\sqrt{q^{2}+\frac{4}{9}|\vec{Q}|^{2}-\frac{4}{3}|\vec{Q}| q x} .
$$

Note that we abbreviate $\hat{a} \equiv 2 a+1$. The isospin factor $I\left(t, T, M_{T}\right)$ arising from the isospin matrix element

$$
\left.\left\langle\left(t \frac{1}{2}\right) T M_{T}\left|G_{E}^{p} \frac{1}{2}\left(1+\hat{\tau}_{z}(1)\right)+G_{E}^{n} \frac{1}{2}\left(1-\hat{\tau}_{z}(1)\right)\right|\left(t^{\prime \prime} \frac{1}{2}\right) T^{\prime \prime} M_{T}^{\prime \prime}\right\rangle\right|_{T^{\prime \prime}=\frac{1}{2}}
$$

is given as

$$
\begin{array}{r}
I\left(t, T, M_{T}\right)=\delta_{M_{T}^{\prime \prime} M_{T}} \delta_{t^{\prime \prime} t}\left[\left(G_{E}^{p}+G_{E}^{n}\right) \frac{1}{2} \delta_{T \frac{1}{2}}\right. \\
\left.-\left(G_{E}^{p}-G_{E}^{n}\right) \sqrt{3}\left(1 \frac{1}{2} T ; 0 M_{T} M_{T}\right)(-1)^{t}\left\{\begin{array}{ccc}
t & \frac{1}{2} & \frac{1}{2} \\
1 & T & \frac{1}{2}
\end{array}\right\}\right] .
\end{array}
$$

The corresponding expressions for the convection and spin currents are

$$
\begin{array}{r}
\left\langle p q \alpha J M ; T M_{T}\left|J_{\tau}^{\text {convect }}(1)\right| \Psi M^{\prime \prime}\right\rangle= \\
(-1)^{\tau} \sqrt{\pi} \frac{q}{m_{N}} I\left(t, T, M_{T}\right)(-1)^{j+\lambda+1} \sqrt{\hat{J}} \sqrt{\hat{I}} \sqrt{\hat{\lambda}} \\
\sum_{\alpha^{\prime \prime}} \delta_{l^{\prime \prime} l} \delta_{s^{\prime \prime} s} \delta_{j^{\prime \prime} j} \delta_{t^{\prime \prime} t} \sqrt{\hat{\lambda^{\prime \prime}}} \sqrt{\hat{I^{\prime \prime}}} \sqrt{\left(2 \lambda^{\prime \prime}+1\right) !} \\
\sum_{\lambda_{1}^{\prime \prime}+\lambda_{2}^{\prime \prime}=\lambda^{\prime \prime}}(q)^{\lambda_{1}^{\prime \prime}}\left(\frac{2}{3} Q\right)^{\lambda_{2}^{\prime \prime}} \frac{1}{\sqrt{\left(2 \lambda_{1}^{\prime \prime}\right) !\left(2 \lambda_{2}^{\prime \prime}\right) !}} \\
\sum_{g_{1}}\left(\lambda 1 g_{1} ; 000\right) \sum_{k} \hat{k}\left(k \lambda_{1}^{\prime \prime} g_{1} ; 000\right) g_{k}
\end{array}
$$




$$
\begin{array}{r}
\sum_{g_{2}}\left(k \lambda_{2}^{\prime \prime} g_{2} ; 000\right)\left\{\begin{array}{ccc}
g_{2} & g_{1} & \lambda^{\prime \prime} \\
\lambda_{1}^{\prime \prime} & \lambda_{2}^{\prime \prime} & k
\end{array}\right\} Y_{g_{2}, M^{\prime \prime}-M+\tau}(\hat{Q}) \\
\sum_{h} \sqrt{\hat{h}}\left\{\begin{array}{ccc}
\lambda & 1 & g_{1} \\
g_{2} & \lambda^{\prime \prime} & h
\end{array}\right\}\left\{\begin{array}{ccc}
I & \lambda & \frac{1}{2} \\
\lambda^{\prime \prime} & I^{\prime \prime} & h
\end{array}\right\}\left\{\begin{array}{ccc}
h & I & I^{\prime \prime} \\
j & \frac{1}{2} & J
\end{array}\right\} \\
\left(J h \frac{1}{2} ; M, M^{\prime \prime}-M, M^{\prime \prime}\right)\left(1 g_{2} h ;-\tau, M^{\prime \prime}-M+\tau, M^{\prime \prime}-M\right)
\end{array}
$$

and

$$
\begin{aligned}
& \left\langle p q \alpha J M ; T M_{T}\left|J_{\tau}^{\text {spin }}(1)\right| \Psi M^{\prime \prime}\right\rangle= \\
& (-3) \sqrt{\pi} \frac{Q}{m_{N}} \tilde{I}\left(t, T, M_{T}\right) \sqrt{\hat{I}} \sqrt{\hat{J}}(-1)^{\frac{1}{2}+I}(-1)^{J+\frac{1}{2}} \\
& \sum_{\alpha^{\prime \prime}} \delta_{l^{\prime \prime} l} \delta_{s^{\prime \prime} s} \delta_{j^{\prime \prime} j} \delta_{t^{\prime \prime} t} \sqrt{\left(2 \lambda^{\prime \prime}+1\right) !} \sqrt{\hat{\lambda}^{\prime \prime}} \sqrt{\hat{I^{\prime \prime}}} \\
& \sum_{\lambda_{1}^{\prime \prime}+\lambda_{2}^{\prime \prime}=\lambda^{\prime \prime}}(q)^{\lambda_{1}^{\prime \prime}}\left(\frac{2}{3} Q\right)^{\lambda_{2}^{\prime \prime}} \frac{1}{\sqrt{\left(2 \lambda_{1}^{\prime \prime}\right) !\left(2 \lambda_{2}^{\prime \prime}\right) !}} \\
& \sum_{k} \hat{k}\left(k \lambda_{1}^{\prime \prime} \lambda ; 000\right) g_{k} \\
& \sum_{g} \sqrt{\hat{g}}\left(k \lambda_{2}^{\prime \prime} g ; 000\right)\left\{\begin{array}{ccc}
g & \lambda & \lambda^{\prime \prime} \\
\lambda_{1}^{\prime \prime} & \lambda_{2}^{\prime \prime} & k
\end{array}\right\}(-1)^{g} \\
& \sum_{f} \hat{f}\left\{\begin{array}{lll}
g & \lambda & \lambda^{\prime \prime} \\
\frac{1}{2} & I^{\prime \prime} & f
\end{array}\right\}\left\{\begin{array}{ccc}
1 & \frac{1}{2} & \frac{1}{2} \\
\lambda & I & f
\end{array}\right\} \\
& \sum_{x} \hat{x}(-1)^{f+x}\left\{\begin{array}{lll}
g & f & I^{\prime \prime} \\
j & \frac{1}{2} & x
\end{array}\right\}\left\{\begin{array}{lll}
1 & f & I \\
j & J & x
\end{array}\right\} \\
& \sum_{w} \hat{w}\left\{\begin{array}{lll}
1 & 1 & 1 \\
g & h & w
\end{array}\right\}\left\{\begin{array}{lll}
J & 1 & x \\
g & \frac{1}{2} & w
\end{array}\right\} \\
& \sum_{h} \frac{1}{\sqrt{\hat{h}}}(g 1 h ; 000)\left(1 w h ; \tau, M^{\prime \prime}-M, M^{\prime \prime}-M+\tau\right) \\
& \left(J w \frac{1}{2} ; M, M^{\prime \prime}-M, M^{\prime \prime}\right) Y_{h, M^{\prime \prime}-M+\tau}(\hat{Q}),
\end{aligned}
$$

where in $\tilde{I}\left(t, T, M_{T}\right)$ the electric nucleon form factors, $G_{E}^{p}$ and $G_{E}^{n}$ are replaced by the magnetic nucleon form factors, $G_{M}^{p}$ and $G_{M}^{n}$.

The second piece in (232) including the permutation operators $P$ is evaluated as

$$
\left\langle p q \alpha\left|P j_{S N}(1)\right| \Psi\right\rangle=£\left\langle p q \alpha|P| p^{\prime} q^{\prime} \alpha^{\prime}\right\rangle\left\langle p^{\prime} q^{\prime} \alpha^{\prime}\left|j_{S N}(1)\right| \Psi\right\rangle
$$


Now the partial wave representation of $P$ can be chosen in various forms [106]. Beside purely geometrical quantities it is always an integral of two $\delta$-functions over the cosine of an angle, where the arguments depend on momenta and that cosine. The two $\delta$-functions express two of the four momenta in terms of the other two. In the case of (242) one chooses the form of $P$ where $p^{\prime}$ and $q^{\prime}$ are expressed in terms of $p$ and $q$ :

$$
\left\langle p q \alpha|P| p^{\prime} q^{\prime} \alpha^{\prime}\right\rangle=\int_{-1}^{1} d x G_{\alpha \alpha^{\prime}}(p, q, x) \frac{\delta\left(p^{\prime}-\pi_{1}(p, q, x)\right)}{p^{\prime l^{\prime}+2}} \frac{\delta\left(q^{\prime}-\pi_{2}(p, q, x)\right)}{q^{\prime \lambda^{\prime}+2}} .
$$

This then leads to

$$
\left\langle p q \alpha\left|P j_{S N}(1)\right| \Psi\right\rangle=\sum_{\alpha^{\prime}} \int_{-1}^{1} d x G_{\alpha \alpha^{\prime}}(p, q, x) \frac{\left\langle\pi_{1}(p, q, x) \pi_{2}(p, q, x) \alpha^{\prime}\left|j_{S N}(1)\right| \Psi\right\rangle}{\pi_{1}(p, q, x)^{l^{\prime}} \pi_{2}(p, q, x)^{\lambda^{\prime}}},
$$

where the functions $G_{\alpha \alpha^{\prime}}(p, q, x), \pi_{1}(p, q, x)$ and $\pi_{2}(p, q, x)$ are given in [106]

Because one evaluates $\left\langle p q \alpha\left|j_{S N}(1)\right| \Psi\right\rangle$ on certain grids in $p$ and $q$, the evaluation of (244) requires interpolation. We use cubic splines of two types [108, 109]. In this manner the driving term in (231) is determined on grids in $p$ and $q$.

We solve the set of coupled integral equations in the two variables $p$ and $q$ by iteration, generating the multiple scattering series for each fixed total angular momentum $J$ and parity. We neglect the coupling of states with total isospin $T=\frac{1}{2}$ and $T=\frac{3}{2}$, which is due to charge independence breaking for np and pp forces but keep both isospins $T$. The difference between pp and np forces is, however, taken into account by applying the " $\frac{2}{3}-\frac{1}{3}$ " rule $[110,111]$. For the lower $J$-values (especially for $J=\frac{1}{2}^{+}$, the $3 \mathrm{~N}$ bound state quantum numbers) that multiple scattering series diverges or converges only very slowly. For every $J^{\pi}$-value we sum up the series by the Padé method [69] which is a very reliable and accurate method. Because of the rather high dimension of the discretized integral kernel an iterative procedure is mandatory. Typical dimensions for the kernel are $100000 \times 100000$ for each $J^{\pi}$-value.

Once $\langle p q \alpha \mid \tilde{U}\rangle$ has been determined, final integrations are required to arrive at the nuclear matrix elements $\left\langle\phi_{q}|P| \tilde{U}\right\rangle$ occurring in (58). In this case another form of the permutation operator is used, namely

$$
\left\langle p q \alpha|P| p^{\prime} q^{\prime} \alpha^{\prime}\right\rangle=\int_{-1}^{1} d x \tilde{G}_{\alpha \alpha^{\prime}}\left(q, q^{\prime}, x\right) \frac{\delta\left(p-\tilde{\pi}_{1}\left(q, q^{\prime}, x\right)\right)}{p^{l+2}} \frac{\delta\left(p^{\prime}-\tilde{\pi}_{2}\left(q, q^{\prime}, x\right)\right)}{p^{l^{\prime}+2}} .
$$


The two $\delta$-functions allow to perform the integrations over $p$ and $p^{\prime}$ and one encounters the deuteron wave function components $\varphi_{l}\left(\tilde{\pi}_{1}\right)(l=0,2)$ and $\left\langle\tilde{\pi}_{2} q^{\prime} \alpha^{\prime} \mid \tilde{U}\right\rangle$ which can be gained by cubic splines interpolation. We refer to $[16,18,71]$ and references therein for the detailed notation.

In the case of the complete breakup one encounters the matrix elements (45), (47) or (55). In the case of $\left\langle\varphi_{0}|P| \tilde{U}\right\rangle$ we use (227) and apply the permutation $P$ to the left. Then we obtain the structure

$$
\left\langle\vec{p}^{\prime} \vec{q}^{\prime} \mid \tilde{U}\right\rangle=\sum\left\langle\vec{p}^{\prime} \vec{q}^{\prime} \mid p q \alpha\right\rangle\langle p q \alpha \mid \tilde{U}\rangle
$$

with certain linear combinations $\vec{p}^{\prime}$ and $\vec{q}^{\prime}$ of the original final momenta $\vec{p}$ and $\vec{q}$. The overlaps $\left\langle\vec{p}^{\prime} \vec{q}^{\prime} \mid p q \alpha\right\rangle$ are trivially given by the very definition of the basis states $\mid p q \alpha>$ in terms of geometrical quantities and spherical harmonics [112].

The remaining term $\left\langle\varphi_{0}\left|t G_{0} P\right| \tilde{U}\right\rangle$ requires just an application of part of the kernel in (44) onto $|\tilde{U}\rangle$ and the additional structures in (44) and (47) are treated in a corresponding manner. We refer to [18] for more details.

The capture matrix element given in (66) consists of two terms. For the second one we need the quantity $T$, which is part of the $3 \mathrm{~N}$ breakup amplitude for $\mathrm{Nd}$ scattering [18] and is determined in form of the set of functions $\langle p q \alpha \mid T\rangle$. The free propagator $G_{0}$ delivers a simple pole, which we treat by subtraction. The remaining part $\langle\Psi|\mathcal{O}(1+P)| p q \alpha\rangle$ has been discussed before. The first term in (66) is apparently closely related to the first term in (59). With these relatively schematic and brief remarks we end the description of the performance related to matrix elements and Faddeev like integral equations. For practitioners more is needed and we refer for details to $[16,18,71,113]$.

Up to now we addressed the $|p q \alpha\rangle$-representation of the single nucleon current. The representation of the two-body currents is much more complex. In the $3 \mathrm{~N}$ space spanned by the Jacobi momenta $\vec{p}$ and $\vec{q}$ the two-body current related to particles 2 and 3 has the form

$$
\left\langle\vec{p}^{\prime} \vec{q}^{\prime}|\vec{j}(2,3)| \vec{p} \vec{q}\right\rangle=\delta\left(\vec{q}^{\prime}-\vec{q}-\frac{1}{3} \vec{Q}\right) \vec{J}\left(\vec{p}_{2}, \vec{p}_{3}\right),
$$

where

$$
\begin{array}{r}
\vec{p}_{2} \equiv \vec{k}_{2}^{\prime}-\vec{k}_{2}=\frac{1}{2} \vec{Q}+\vec{p}^{\prime}-\vec{p}, \\
\vec{p}_{3} \equiv \vec{k}_{3}^{\prime}-\vec{k}_{3}=\frac{1}{2} \vec{Q}-\vec{p}^{\prime}+\vec{p},
\end{array}
$$


are the momentum transfers to nucleons 2 and 3. The photon momentum $\vec{Q}$ occurs due to the overall momentum conserving $\delta$-function. The structure (247) shows that the $\vec{p}$ and $\vec{q}$ dependence is separated, what simplifies the partial wave decomposition quite substantially. Let us split the basis states as

$$
|p q \alpha\rangle=\left|p q(l s) j\left(\lambda \frac{1}{2}\right) I(j I) J m\right\rangle\left|\left(t \frac{1}{2}\right) T m_{T}\right\rangle \equiv\left|p q \alpha_{J}\right\rangle\left|\alpha_{T}\right\rangle,
$$

and introduce

$$
\mathcal{Y}_{j \mu}(\hat{p}) \equiv \sum_{\mu^{\prime}} C\left(l s j ; \mu-\mu^{\prime}, \mu^{\prime}, \mu\right) Y_{l, \mu-\mu^{\prime}}(\hat{p})\left|s \mu^{\prime}\right\rangle
$$

and

$$
\mathcal{Y}_{\lambda \nu}(\hat{q}) \equiv \sum_{\nu^{\prime}} C\left(\lambda \frac{1}{2} I ; \nu-\nu^{\prime}, \nu^{\prime}, \nu\right) Y_{\lambda, \nu-\nu^{\prime}}(\hat{q})\left|\frac{1}{2} \nu^{\prime}\right\rangle
$$

Then

$$
\begin{aligned}
\left\langle p^{\prime} q^{\prime} \alpha^{\prime}|\vec{j}(2,3)| p q \alpha\right\rangle & =\int d \overrightarrow{p_{1}^{\prime}} \int d \overrightarrow{q_{1}^{\prime}} \int d \overrightarrow{p_{1}} \int d \overrightarrow{q_{1}}\left\langle p^{\prime} q^{\prime} \alpha \mid \overrightarrow{p_{1}^{\prime}} \overrightarrow{q_{1}^{\prime}}\right\rangle\left\langle\overrightarrow{p_{1}^{\prime}} \overrightarrow{q_{1}^{\prime}}|j(2,3)| \overrightarrow{p_{1}} \overrightarrow{q_{1}}\right\rangle\left\langle\overrightarrow{p_{1}} \overrightarrow{q_{1}} \mid p q \alpha\right\rangle \\
& =\int d \overrightarrow{p_{1}^{\prime}} \int d \overrightarrow{q_{1}^{\prime}} \int d \overrightarrow{p_{1}} \int d \overrightarrow{q_{1}} \sum_{\mu^{\prime} \mu} C\left(j^{\prime} I^{\prime} J^{\prime} ; \mu^{\prime}, M^{\prime}-\mu^{\prime}, M^{\prime}\right) C(j I J ; \mu, M-\mu, M) \\
& \times \mathcal{Y}_{j^{\prime} \mu^{\prime}}^{*}\left(\hat{p_{1}^{\prime}}\right) \frac{\delta\left(p_{1}^{\prime}-p^{\prime}\right)}{p_{1}^{\prime 2}} \mathcal{Y}_{I^{\prime}, M^{\prime}-\mu^{\prime}}^{*}\left(\hat{q_{1}^{\prime}}\right) \frac{\delta\left(q_{1}^{\prime}-q^{\prime}\right)}{q_{1}^{\prime 2}} \delta\left(\overrightarrow{q_{1}}-\overrightarrow{q_{1}^{\prime}}-\frac{1}{3} \vec{Q}\right) \\
& \times\left\langle\alpha_{T^{\prime}}\left|\vec{j}\left(\frac{1}{2} \vec{Q}+\vec{p}_{1}^{\prime}-\vec{p}_{1}, \frac{1}{2} \vec{Q}-\vec{p}_{1}^{\prime}+\vec{p}_{1} ; 2,3\right)\right| \alpha_{T}\right\rangle \\
& \times \mathcal{Y}_{j \mu}\left(\hat{p_{1}}\right) \mathcal{Y}_{I, M-\mu}\left(\hat{q_{1}}\right) \frac{\delta\left(p_{1}-p\right)}{p_{1}^{2}} \frac{\delta\left(q_{1}-q\right)}{q_{1}^{2}} .
\end{aligned}
$$

Here $\vec{p}_{1}, \vec{q}_{1}$ and $\vec{p}_{1}^{\prime}, \vec{q}_{1}^{\prime}$ are Jacobi momenta of the type (23), what leads immediately to

$$
\begin{aligned}
\left\langle p^{\prime} q^{\prime} \alpha^{\prime}|\vec{j}(2,3)| p q \alpha\right\rangle & \\
& =\sum_{\mu^{\prime} \mu} C\left(j^{\prime} I^{\prime} J^{\prime} ; \mu^{\prime}, M^{\prime}-\mu^{\prime}, M^{\prime}\right) C(j I J ; \mu, M-\mu, M) \\
& \times \vec{I}_{2}\left(p^{\prime}, p, Q ;\left(l^{\prime} s^{\prime}\right) j^{\prime} \mu^{\prime} \alpha_{T^{\prime}},(l s) j \mu \alpha_{T}\right) \\
& \times I_{3}\left(q^{\prime}, q, Q ;\left(\lambda^{\prime} \frac{1}{2}\right) I^{\prime} M^{\prime}-\mu^{\prime},\left(\lambda \frac{1}{2}\right) I M-\mu\right),
\end{aligned}
$$

with

$$
\begin{array}{r}
\vec{I}_{2}\left(p^{\prime}, p, Q ;\left(l^{\prime} s^{\prime}\right) j^{\prime} \mu^{\prime} \alpha_{T^{\prime}},(l s) j \mu, \alpha_{T}\right) \\
=\int d \hat{p^{\prime}} \int d \hat{p} \mathcal{Y}_{j^{\prime} \mu^{\prime}}^{*}\left(\hat{p^{\prime}}\right)\left\langle\alpha_{T^{\prime}}\left|\vec{j}\left(\frac{1}{2} \vec{Q}+\vec{p}^{\prime}-\vec{p}, \frac{1}{2} \vec{Q}-\vec{p}^{\prime}+\vec{p} ; 2,3\right)\right| \alpha_{T}\right\rangle \mathcal{Y}_{j \mu}(\hat{p}),
\end{array}
$$


and

$$
\begin{aligned}
& I_{3}\left(q^{\prime}, q, Q ;\left(\lambda^{\prime} \frac{1}{2}\right) I^{\prime} M^{\prime}-\mu^{\prime},\left(\lambda \frac{1}{2}\right) I M-\mu\right) \\
& =\int d \hat{q}^{\prime} \mathcal{Y}_{I^{\prime}, M^{\prime}-\mu^{\prime}}^{*}\left(\hat{q}^{\prime}\right) \frac{\delta\left(q-\left|\overrightarrow{q^{\prime}}+\frac{1}{3} \vec{Q}\right|\right)}{q^{2}} \mathcal{Y}_{I, M-\mu}\left(\overrightarrow{q^{\prime}}+\widehat{\frac{1}{3}} \vec{Q}\right) \text {. }
\end{aligned}
$$

In the nuclear matrix elements the current is applied onto the ${ }^{3} \mathrm{He}$ state. Therefore we need the basic building blocks $\langle p q \alpha|\vec{j}(2,3)| \Psi\rangle$. We obtain

$$
\begin{array}{r}
\left\langle p^{\prime} q^{\prime} \alpha^{\prime}|\vec{j}(2,3)| \Psi\right\rangle=\sum_{\alpha} \int p^{2} d p q^{2} d q\left\langle p^{\prime} q^{\prime} \alpha^{\prime}|\vec{j}(2,3)| p q \alpha\right\rangle\langle p q \alpha \mid \Psi\rangle \\
=\sum_{\alpha} \int p^{2} d p \sum_{\mu \mu^{\prime}} C\left(j^{\prime} I^{\prime} J^{\prime} ; \mu^{\prime}, M^{\prime}-\mu^{\prime}, M^{\prime}\right) C(j I J, \mu ; M-\mu, M) \\
\times \vec{I}_{2}\left(p^{\prime}, p, Q ;\left(l^{\prime} s^{\prime}\right) j^{\prime} \mu^{\prime} \alpha_{T^{\prime}},(l s) j \mu \alpha_{T}\right) \\
\times \tilde{I}_{3}\left(p, q^{\prime}, Q ;\left(\lambda^{\prime} \frac{1}{2}\right) I^{\prime} M^{\prime}-\mu^{\prime},\left(\lambda \frac{1}{2}\right) I M-\mu\right),
\end{array}
$$

with

$$
\begin{array}{r}
\tilde{I}_{3}\left(p, q^{\prime}, Q ;\left(\lambda^{\prime} \frac{1}{2}\right) I^{\prime} M^{\prime}-\mu^{\prime},\left(\lambda \frac{1}{2}\right) I M-\mu\right)= \\
\int d \hat{q^{\prime}} \mathcal{Y}_{I^{\prime}, M^{\prime}-\mu^{\prime}}^{*}\left(\hat{q^{\prime}}\right)\left\langle p,\left|\overrightarrow{q^{\prime}}+\frac{1}{3} \vec{Q}\right|, \alpha \mid \Psi\right\rangle \mathcal{Y}_{I, M-\mu}\left(\overrightarrow{q^{\prime}}+\frac{1}{3} \vec{Q}\right) .
\end{array}
$$

The angular integration in $\tilde{I}_{3}$ can be performed by well established analytical steps (see [69]). The much harder task is the reliable evaluation of $\vec{I}_{2}$. It is convenient to decompose the current as

$$
\vec{j}(2,3)=G_{E}^{V} \sum_{k \kappa} \vec{O}^{k \kappa}\left(\vec{p}_{2}, \vec{p}_{3}\right)\{\sigma(2) \otimes \sigma(3)\}_{k \kappa} i[\vec{\tau}(2) \times \vec{\tau}(3)]_{z} .
$$

The $\pi$ - and $\rho$-like currents given in (118) and (123) are of that type. The complex angular momentum algebra is detailed in [107] and we refer the reader to that reference. In [107] we also evaluate those integrals directly in a numerical manner to check the validity and accuracy independently. Benchmark studies are displayed there, which we think are very useful for practitioners, since the momentum space representation of the two-body currents requires great care. 


\section{COMPARISON WITH DATA}

Our theoretical framework is nonrelativistic. This limits the range of data we can analyze. Unfortunately, in addition, quite a few data are not well documented in the literature with respect to the necessary angular and energy averaging. Therefore, a quantitative comparison of such data to our theory is no longer possible. Under all these limitations we are aware of only a restricted data set, which we shall display now.

In the following, the dynamical input for the theoretical calculations is always the NN force AV18 alone or together with the 3N force UrbanaIX [114]. Including the 3N force, the resulting binding energies for ${ }^{3} \mathrm{He}$ and ${ }^{3} \mathrm{H}$ are 7.746 and $8.476 \mathrm{MeV}$, respectively, which is sufficiently close to the experimental values (7.718109 \pm 0.000010$) \mathrm{MeV}$ and (8.481855 \pm 0.000013$) \mathrm{MeV}[115]$. The AV18 potential includes electromagnetic forces $[15,54]$. They are all kept in our treatment of the two $3 \mathrm{~N}$ bound states but for the $3 \mathrm{~N}$ continuum we keep only the strong forces.

On top of the standard single nucleon current, we employ the $\pi$ - and $\rho$-like two-body currents related to AV18. In the case of photodisintegration we also show examples based on the Siegert approximation as defined in section IIIB. Technically we still rely on a partial wave decomposition which is always converged within our typical numerical accuracy of about $1-2 \%$ in the observables.

\section{A. Elastic electron scattering on ${ }^{3} \mathrm{He}$ and ${ }^{3} \mathrm{H}$}

It has been known for a long time $[116,117]$ that the $3 \mathrm{~N}$ charge and magnetic form factors require two-body densities and two-body currents. The two-body density is already a relativistic correction and therefore strictly spoken already outside our framework. Nevertheless, we follow [89] and use the one- $\pi$ and one- $\rho$ exchange process. Nowadays [26] the radial functions " $v$ " are also taken from the $\pi$ - and $\rho$-like pieces of AV18. In all calculations the UrbanaIX $3 \mathrm{NF}$ is included.

Our results for the charge form factors of ${ }^{3} \mathrm{He}$ and ${ }^{3} \mathrm{H}$ are shown in Figs. 7 and 8. The dashed curves are based on the single nucleon density, solely given by $\mathrm{G}_{E}$ (not including the Darwin-Foldy and spin-orbit terms as in (78)). They start to deviate strongly from the data for momenta above $\approx 2.5 \mathrm{fm}^{-1}$. The solid curve includes in addition to the Darwin-Foldy 
and spin-orbit terms the two-body $\pi-\rho$ densities. All that shifts theory rather close into the data for ${ }^{3} \mathrm{H}$. This is also true at least up to about $3 \mathrm{fm}^{-1}$ for ${ }^{3} \mathrm{He}$. Since in this review we concentrate on a regime which can be called dominantly nonrelativistic we do not comment on missing dynamics responsible for the strong deviations above around $3 \mathrm{fm}^{-1}$ in ${ }^{3} \mathrm{He}$ and on the possibly accidental agreement for ${ }^{3} \mathrm{H}$. Nevertheless, we would like to illustrate the effects of relativity in the single nucleon density operator in Figs. 9-10. Since for $Q$-values below about $3 \mathrm{fm}^{-1}$ the changes in $\mathrm{F}_{C}$ going beyond the single density operator $\mathrm{G}_{E}$ caused by relativity stay below about $5 \%$, we show only the effects for the higher $Q$-values. There we can choose a linear scale and display five curves according to different choices of the single nucleon density operator. We see in Fig. 9 for ${ }^{3}$ He that the Darwin-Foldy term added to the nonrelativistic single nucleon current operator $\left(G_{E}\right)$ shifts the theory downwards, while further adding the spin-orbit term reduces that downward shift. We also display the full relativistic result according to the first term (spin independent) in (70). In the maximum this is identical to the nonrelativistic result. Thus the terms additional to the Darwin Foldy term cancel its contribution completely in this case. Finally, the complete relativistic single nucleon density operator shifts the theory upwards beyond the nonrelativistic result by about $5 \%$ in the maximum.

In the case of ${ }^{3} \mathrm{H}$ both the Darwin-Foldy and the spin-orbit terms shift theory downwards from the nonrelativistic result and the full relativistic curve ends up below the nonrelativistic one by about $14 \%$ in the maximum.

After this small excursion into relativistic features, we display noticeable effects of the $3 \mathrm{NF}$ in Figs. 11-12. For both nuclei, ${ }^{3} \mathrm{He}$ and ${ }^{3} \mathrm{H}$, the addition of the $3 \mathrm{NF}$ shifts the theory closer into the data for the lower $Q$-range, on which we concentrate. For ${ }^{3}$ He the effects grow with $Q$ from 0 to about $12 \%$ at $Q=2 \mathrm{fm}^{-1}$ and about $20 \%$ at $Q=3 \mathrm{fm}^{-1}$. For ${ }^{3} \mathrm{H}$ they are slightly smaller $\left(17 \%\right.$ at $\left.Q=3 \mathrm{fm}^{-1}\right)$. $3 \mathrm{NF}$ effects on the charge form factor have been investigated earlier in [118] showing a similar tendency.

Since at higher $Q$-values the comparison between theory and experiment differs in quality for ${ }^{3} \mathrm{He}$ and ${ }^{3} \mathrm{H}$, it is common to look into the isoscalar and isovector charge form factors defined as

$$
F_{C}^{S, V}=\frac{1}{2}\left[2 F_{C}\left({ }^{3} H e\right) \pm F_{C}\left({ }^{3} H\right)\right] .
$$

They are displayed in Figs. 13-14 together with the data. For the lower $Q$-values the 


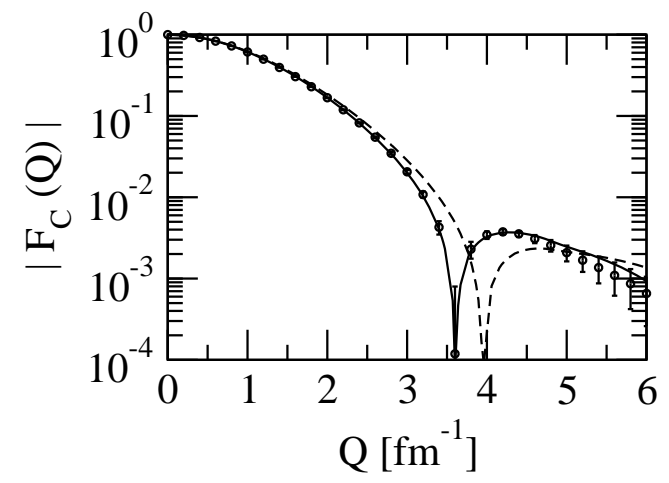

FIG. 7: The charge form factor of ${ }^{3} \mathrm{H}$ as a function of $Q \equiv \sqrt{Q^{2}}$ for the single nucleon density given alone by $G_{E}$ (dashed curve) and including the Darwin-Foldy and spin-orbit terms as well as the two-body $\pi$ - and $\rho$-like densities (solid curve). Data are from [122].

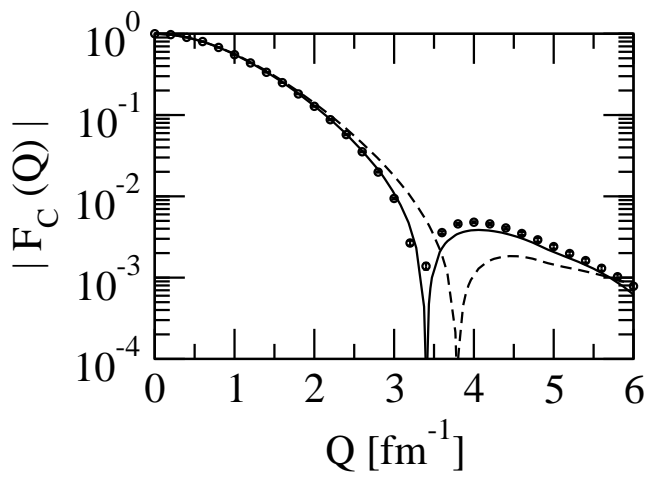

FIG. 8: The same as in Fig. 7, now for ${ }^{3}$ He. Data are from [122].

agreement with the data is in both cases quite good but in the higher $Q$-range the isovector form factor, which is sensitive to our two-body density, underestimates the data significantly. We refer the reader to [119] and [26] for further discussions on that higher $Q$-range and an inclusion of different components of the charge density operator. Including additional parts in the two-body density in [119] leads to a remarkably good description of the data. Similarly, the Hanover group could describe the data very well with a single $\Delta$-isobar admixture and including several selected relativistic corrections $[67,120]$. In [121] the first time threenucleon currents related to the $2 \pi$-exchange $3 \mathrm{NF}$ have been included. Also variational Monte Carlo techniques based on realistic NN and 3N forces have been successfully applied and similar results for the elastic form factors have been achieved [123]. We also would like 


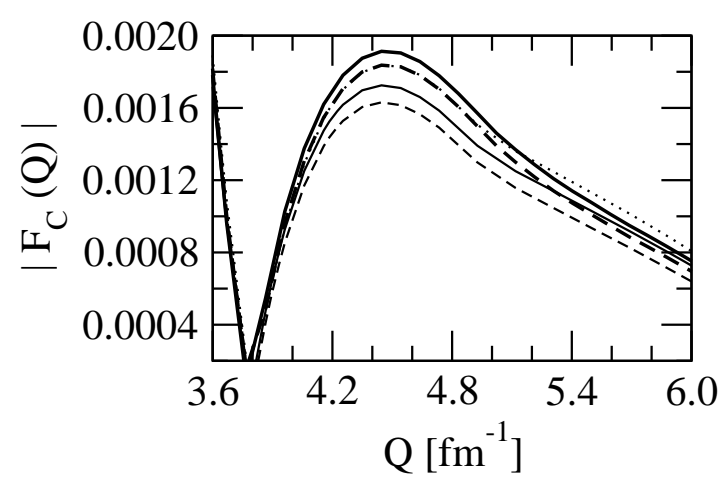

FIG. 9: The effects of relativity in the single nucleon density for the charge form factor of ${ }^{3} \mathrm{He} . G_{E}$ alone (thin dotted), $G_{E}+$ Darwin-Foldy (thin dashed), $G_{E}+$ Darwin-Foldy + spin-orbit (thin solid), the first spin independent term in (70) (thick dashed), the full relativistic density (thick solid).

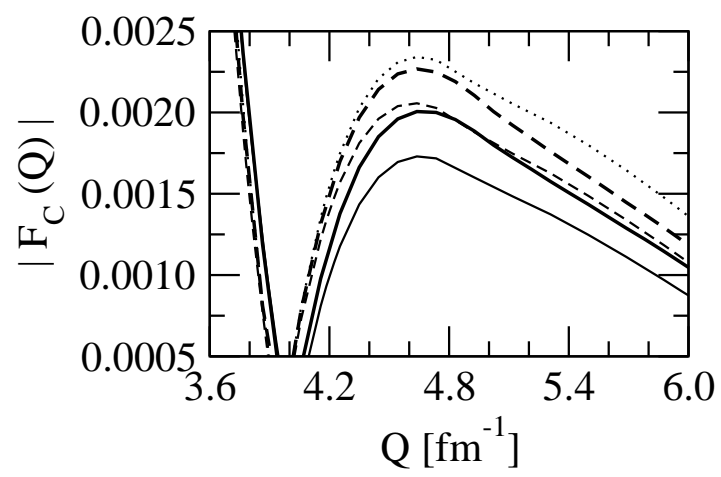

FIG. 10: The effects of relativity in the single nucleon density for the charge form factor of ${ }^{3} \mathrm{H}$. Curves as in Fig. 9.

to draw attention to the work in [124] where relations between isoscalar charge form factors of two- and three-nucleon systems were studied and inconsistencies were found using the "standard" model of meson exchange currents.

Now we regard the magnetic form factors of ${ }^{3} \mathrm{He}$ and ${ }^{3} \mathrm{H}$ in Figs. 15 and 16 . Here the situation is more demanding in relation to the choice of the two-body current operators. Up to about $2.5 \mathrm{fm}^{-1}$ the agreement with the data is quite good but beyond that it is very insufficient. The effects of the $3 \mathrm{NF}$ slightly improve the agreement in the lower $Q$-range as displayed in Figs. 17 and 18. In Fig. 19 we show the isoscalar magnetic form factor which 

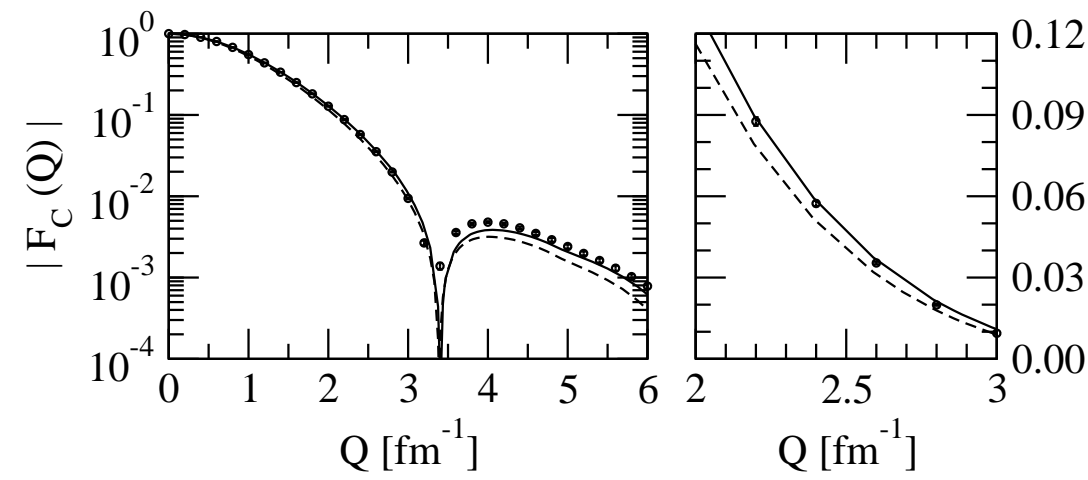

FIG. 11: The 3NF effects for the charge form factor of ${ }^{3} \mathrm{He}$. The solid curve is the same as in Fig. 8. For the dashed curve only the $3 \mathrm{NF}$ has been dropped in the bound state wave function. In the right panel the $Q$-range is restricted to $2 \leq Q \leq 3$ and the linear scale for $F_{C}(Q)$ is used.
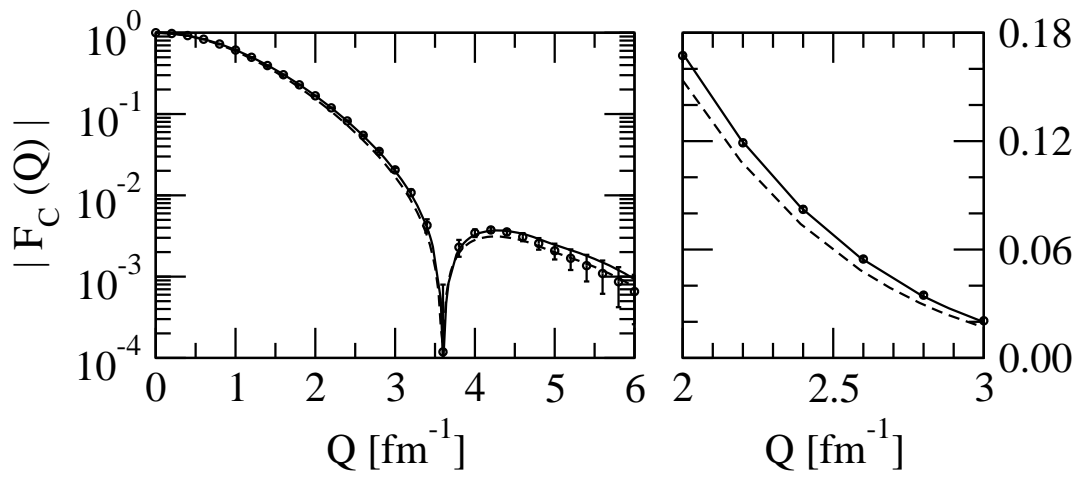

FIG. 12: The 3NF effects for the charge form factor of ${ }^{3} \mathrm{H}$. Curves as in Fig. 11.

is in quite good agreement with the data up to about $4 \mathrm{fm}^{-1}$, while the two-body current dependent isovector magnetic form factor is dramatically off the data in the higher $Q$-range as shown in Fig. 20.

Finally we come to the relativistic effects in the single nucleon current operator given in (71). As in all our results we choose the laboratory frame for which the total momentum of the initial ${ }^{3} \mathrm{He}$ is zero and work with the Jacobi momenta defined in (20) and (21). Assuming that the photon couples to nucleon 1 , the initial $(\vec{p})$ and final $\left(\vec{p}^{\prime}\right)$ individual momenta of the struck nucleon are given in terms of the Jacobi momentum $\vec{q}$ and the three-momentum transfer $\vec{Q}$ as

$$
\vec{p}=\vec{q}-\frac{2}{3} \vec{Q}
$$




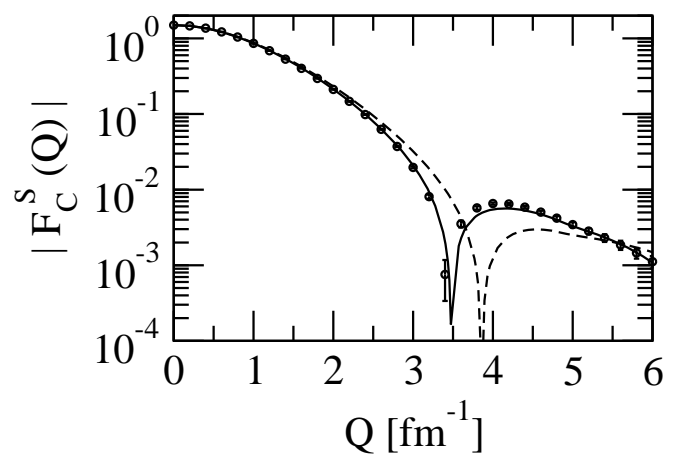

FIG. 13: The 3N isoscalar charge form factor. Curves as in Fig. 7.

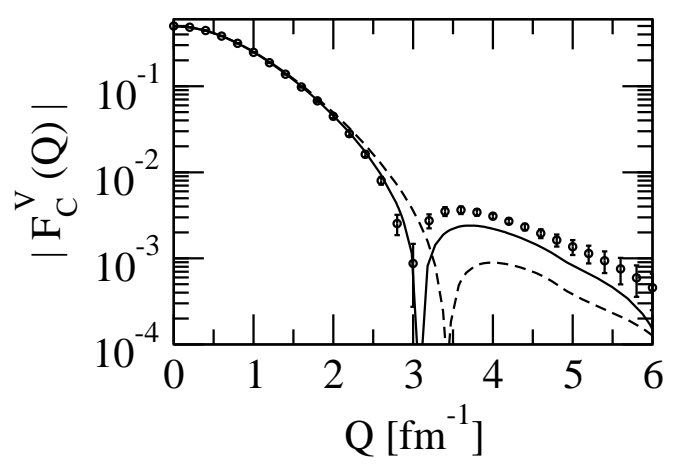

FIG. 14: The 3N isovector charge form factor. Curves as in Fig. 7.

$$
\vec{p}^{\prime}=\vec{q}+\frac{1}{3} \vec{Q} .
$$

Further we put $\vec{Q} \| \hat{z}$, which simplifies the calculation of the spherical $\tau= \pm 1$ components of the current operator given in (71). Since

$$
\begin{gathered}
\left(\vec{p}^{\prime}+\vec{p}\right)_{\tau}=2(\vec{q})_{\tau}, \\
\left(\frac{\vec{p}^{\prime}}{p_{0}+m_{N}}+\frac{\vec{p}}{p_{0}^{\prime}+m_{N}}\right)_{\tau}=\left(\frac{1}{p_{0}+m_{N}}+\frac{1}{p_{0}^{\prime}+m_{N}}\right)(\vec{q})_{\tau}, \\
(\vec{p} \times \vec{\sigma})_{\tau}=(\vec{\sigma} \times \vec{q})_{\tau}-\frac{2}{3}(\vec{Q} \times \vec{\sigma})_{\tau},
\end{gathered}
$$

and

$$
\left(\vec{\sigma} \times \vec{p}^{\prime}\right)_{\tau}=(\vec{\sigma} \times \vec{q})_{\tau}+\frac{1}{3}(\vec{\sigma} \times \vec{Q})_{\tau}
$$




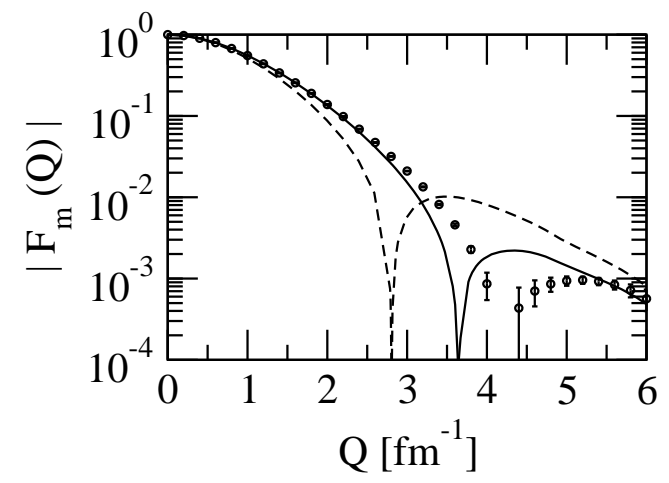

FIG. 15: The magnetic form factor of ${ }^{3} \mathrm{He}$. The dashed line represents the results obtained with the nonrelativistic single nucleon current operator from (72) (with $F_{1}$ replaced by $G_{E}$ ) and the solid line includes the effects of the $\pi$ - and $\rho$-like meson exchange currents. Data are from [122].

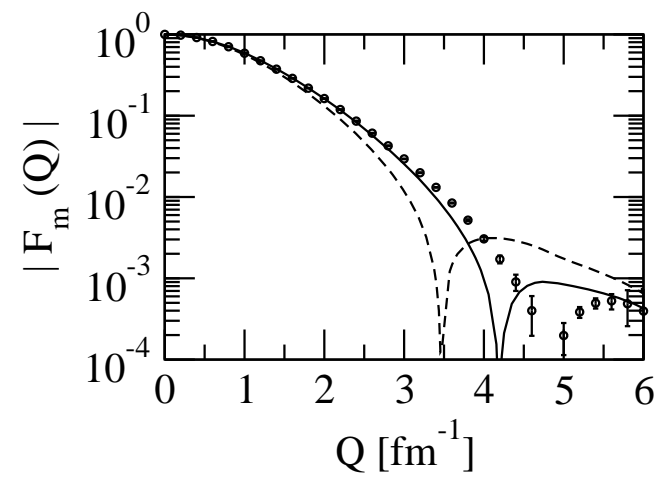

FIG. 16: The magnetic form factor of ${ }^{3} \mathrm{H}$. Curves as in Fig. 15. Data are from [122].

we rewrite $(71)$ as

$$
\begin{array}{r}
J_{\tau}=A\left\{-2 F_{2}\left(1-\frac{\vec{p}^{\prime} \cdot \vec{p}}{\left(p_{0}+m_{N}\right)\left(p_{0}^{\prime}+m_{N}\right)}\right)+G_{M}\left(\frac{1}{p_{0}+m_{N}}+\frac{1}{p_{0}^{\prime}+m_{N}}\right)\right\}(\vec{q})_{\tau} \\
+2 A F_{2} \frac{1}{\left(p_{0}+m_{N}\right)\left(p_{0}^{\prime}+m_{N}\right)} i \vec{\sigma} \cdot(\vec{Q} \times \vec{q})(\vec{q})_{\tau} \\
+A G_{M}\left(\frac{1}{\left(p_{0}+m_{N}\right)}-\frac{1}{\left(p_{0}^{\prime}+m_{N}\right)}\right) i(\vec{q} \times \vec{\sigma})_{\tau} \\
+A G_{M}\left(\frac{2}{3} \frac{1}{\left(p_{0}+m_{N}\right)}+\frac{1}{3} \frac{1}{\left(p_{0}^{\prime}+m_{N}\right)}\right) i(\vec{\sigma} \times \vec{Q})_{\tau} .
\end{array}
$$

The first and the last parts in (266) correspond in the non-relativistic limit to the convection and spin current, respectively. The second and the third parts disappear in the non-relativistic limit and turn out to be less important. This is shown in Figs. 21 (22) for 


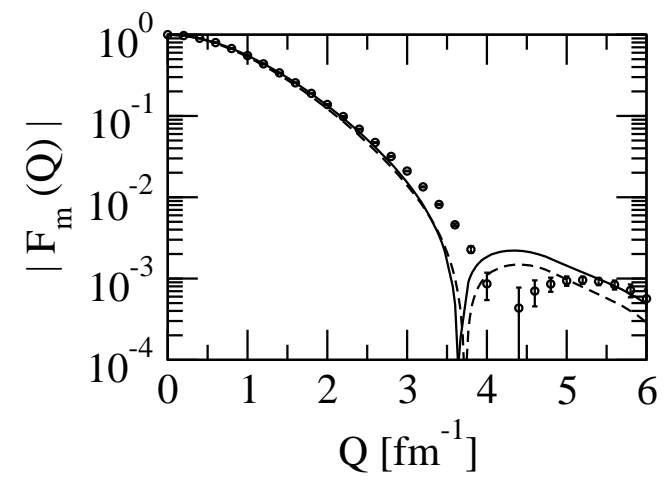

FIG. 17: The $3 \mathrm{NF}$ effects for the magnetic form factor of ${ }^{3} \mathrm{He}$. The solid curve is the same as in Fig. 15. In the case of the dashed curve only the $3 \mathrm{NF}$ has been dropped in the bound state wave function.

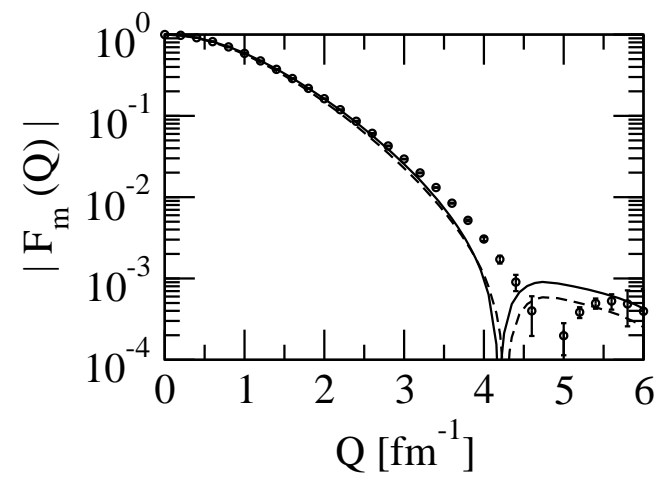

FIG. 18: The 3NF effects for the magnetic form factor of ${ }^{3} \mathrm{H}$. Curves as in Fig. 17.

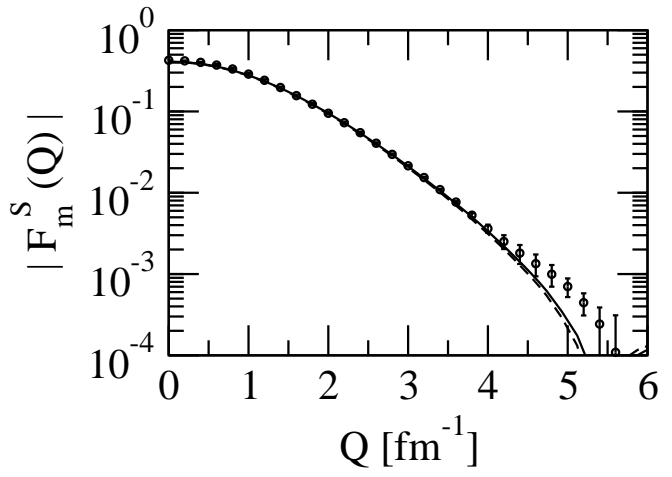

FIG. 19: The 3N isoscalar magnetic form factor. The curves as in Fig. 15. 


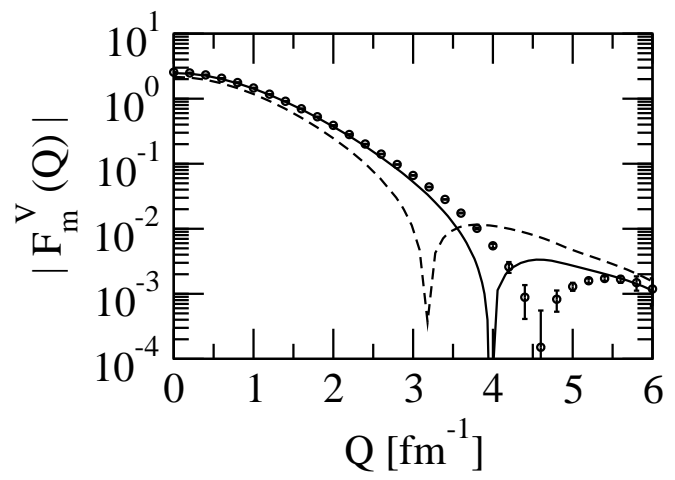

FIG. 20: The 3N isovector magnetic form factor. The curves as in Fig. 15.

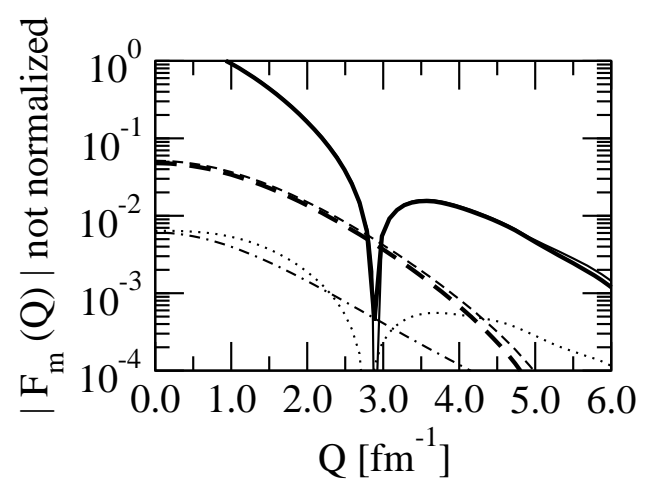

FIG. 21: The convection (thin dashed) and the spin part (thin solid) of the nonrelativistic single nucleon current, and the four parts of the relativistic single nucleon current given in (266) (first (thick dashed), second (dot-dashed), third (dotted), fourth (thick solid) ) for the magnetic form factor of ${ }^{3} \mathrm{He}$. The thin and thick solid lines practically overlap.

${ }^{3} \mathrm{He}\left({ }^{3} \mathrm{H}\right)$. We see that the convection part, nonrelativistically and relativistically, is unimportant. It is the spin part which provides the dominant contribution and the relativistic effects are quite insignificant.

The magnetic form factors have been studied by other groups as well [67, 120, 121], where more sophisticated currents and $\Delta$-admixtures have been included. This shifts theory much closer to the data, especially at the higher $Q$-values, which are not in the focus of this review. Therefore, we do not comment further on all that. Finally, we would like to draw attention to a first attempt within the Bethe-Salpeter approach in the Faddeev form [125] which, however, due to severe truncations cannot yet been conclusively confronted to data. 


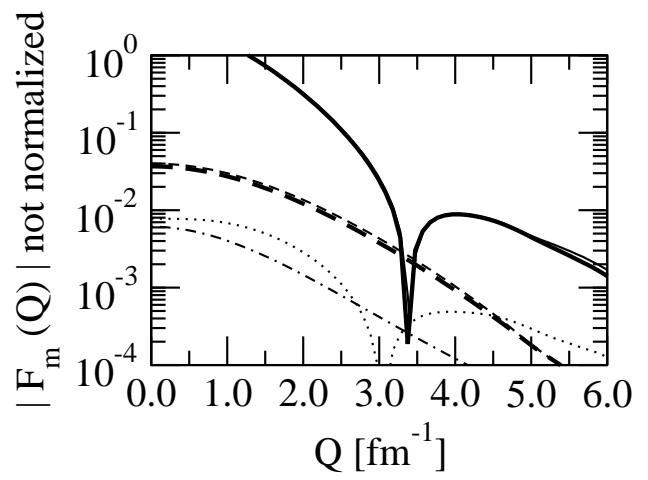

FIG. 22: The same as in Fig. 21 but for ${ }^{3} \mathrm{H}$.

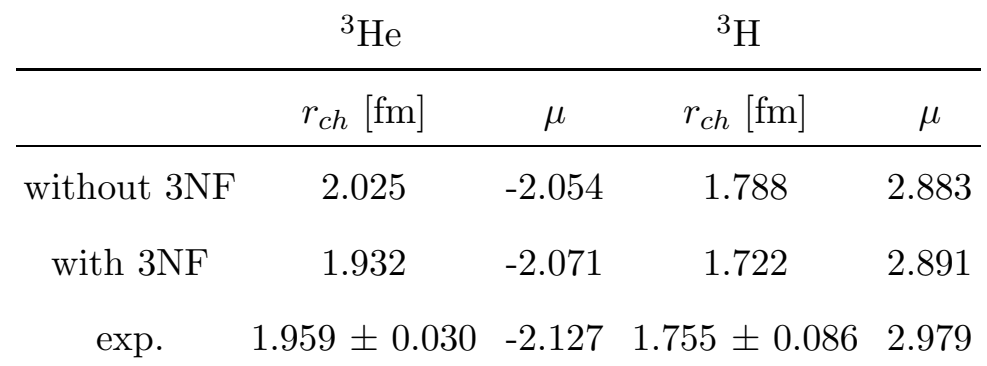

TABLE I: The theoretical predictions including MEC and experimental values for the ${ }^{3} \mathrm{He}$ and ${ }^{3} \mathrm{H}$ charge radii and magnetic moments. Data are from [26, 122] and [126].

We end up with showing the charge radii and the magnetic moments of ${ }^{3} \mathrm{He}$ and ${ }^{3} \mathrm{H}$ in Table I. In all cases the inclusion of the 3NF improves the description of the data. Small discrepancies remain. The agreement in the case of the magnetic moments is somewhat better using the enriched dynamics in $[67,121]$.

\section{B. Inclusive electron scattering on ${ }^{3} \mathrm{He}$ and ${ }^{3} \mathrm{H}$}

Without polarization two response functions $R_{L}$ and $R_{T}$ defined in (170)-(172) can be measured using a Rosenbluth separation method. We compare in Figs. 23 and 24 for ${ }^{3} \mathrm{H}$ and ${ }^{3} \mathrm{He}$ the data to our theory for the longitudinal response function $R_{L}$ depending on the energy transfer $\omega=Q_{0}$ at the $|\vec{Q}|$-values 200, 300, 400 and $500 \mathrm{MeV} / \mathrm{c}$. As can be seen already at $|\vec{Q}|=500 \mathrm{MeV} / \mathrm{c}$ the experimental and theoretical peak positions are slightly different. This is already the result of our non-relativistic kinematics and could be cured by improving the kinematics. We have not done that and will concentrate on the lower 
$|\vec{Q}|$-values.

The two types of plane wave approximations, PWIA and PWIAS (not shown), are very much off the data at 200 and $300 \mathrm{MeV} / \mathrm{c}$. There the inclusion of the rescattering in the final state is strongly needed. We would like to point out, that we distinguish between final state interaction effects when the nucleons in the final state are interacting only through NN forces (FSI) and when both two- and three-nucleon forces are acting. In the following, we also present the results of the simplified treatment of FSI, where the interaction is restricted only to the spectator nucleons 2 and 3 (FSI23). A more detailed explanation of FSI23 is given in section VII C. For both nuclei, the FSI predictions are close to the data at $|\vec{Q}|=$ 200 and $300 \mathrm{MeV} / \mathrm{c}$. The effects of the two-body density are marginal in case of the ${ }^{3} \mathrm{H}$, but noticeable for ${ }^{3} \mathrm{He}$. The $3 \mathrm{NF}$ effects are clearly visible. Note that $3 \mathrm{NF}$ effects are taken consistently into account, in the bound and in the scattering states. For the PWIA, FSI23, FSI, FSI+MEC results the $3 \mathrm{~N}$ bound states obtained without $3 \mathrm{NF}$ are used. In the case of ${ }^{3} \mathrm{H}$ the $3 \mathrm{~N}$ force effects lower theory too much and lead to an underprediction of the data while for ${ }^{3}$ He theory goes right away into the data. The underprediction of theory in the case of ${ }^{3} \mathrm{H}$ is clearly visible at $\mathrm{Q}=400 \mathrm{MeV} / \mathrm{c}$. It is also of interest to notice the tendency that the nuclear interaction effects in the continuum decrease with increasing $|\vec{Q}|$-values.

The situation in the case of the transverse response function $R_{T}$, shown in Figs. 25 and 26 is different. The tendency that the interaction effects in the continuum decrease with increasing $|\vec{Q}|$-values starts earlier than for $R_{L}$. Further, the MEC effects are quite strong, as is well known, but are essentially compensated by the $3 \mathrm{NF}$ effects in the maxima. In the lower and upper energy wings of the peaks the addition of the $3 \mathrm{NF}$ has little effect. Overall the agreement of data and theory for our complete prediction (NN and $3 \mathrm{~N}$ forces plus MEC) is quite good for both nuclei, ${ }^{3} \mathrm{H}$ and ${ }^{3} \mathrm{He}$, at $|\vec{Q}|=200,300$ and $400 \mathrm{MeV} / \mathrm{c}$. The very interesting interplay of $3 \mathrm{NF}$ and $\mathrm{MEC}$ effects would make a renewed, more precise measurement very interesting. Finally, like for $R_{L}$, relativistic effects, at least the ones of kinematical origin, are clearly visible at $|\vec{Q}|=500 \mathrm{MeV} / \mathrm{c}$.

There are also inclusive data [129] for $|\vec{Q}|=174,323$ and $487 \mathrm{MeV} / \mathrm{c}$, starting at threshold. They are plotted as a function of the energy transfer $\omega$ in Figs. 27- 30 in comparison to our theory. The overall agreement of our complete theory with the data is quite good, for both nuclei, ${ }^{3} \mathrm{H}$ and ${ }^{3} \mathrm{He}$. Not including full FSI would be a disaster for all $|\vec{Q}|$-values: namely predictions based on the two simplest approximations, PWIA and FSI23, are far 

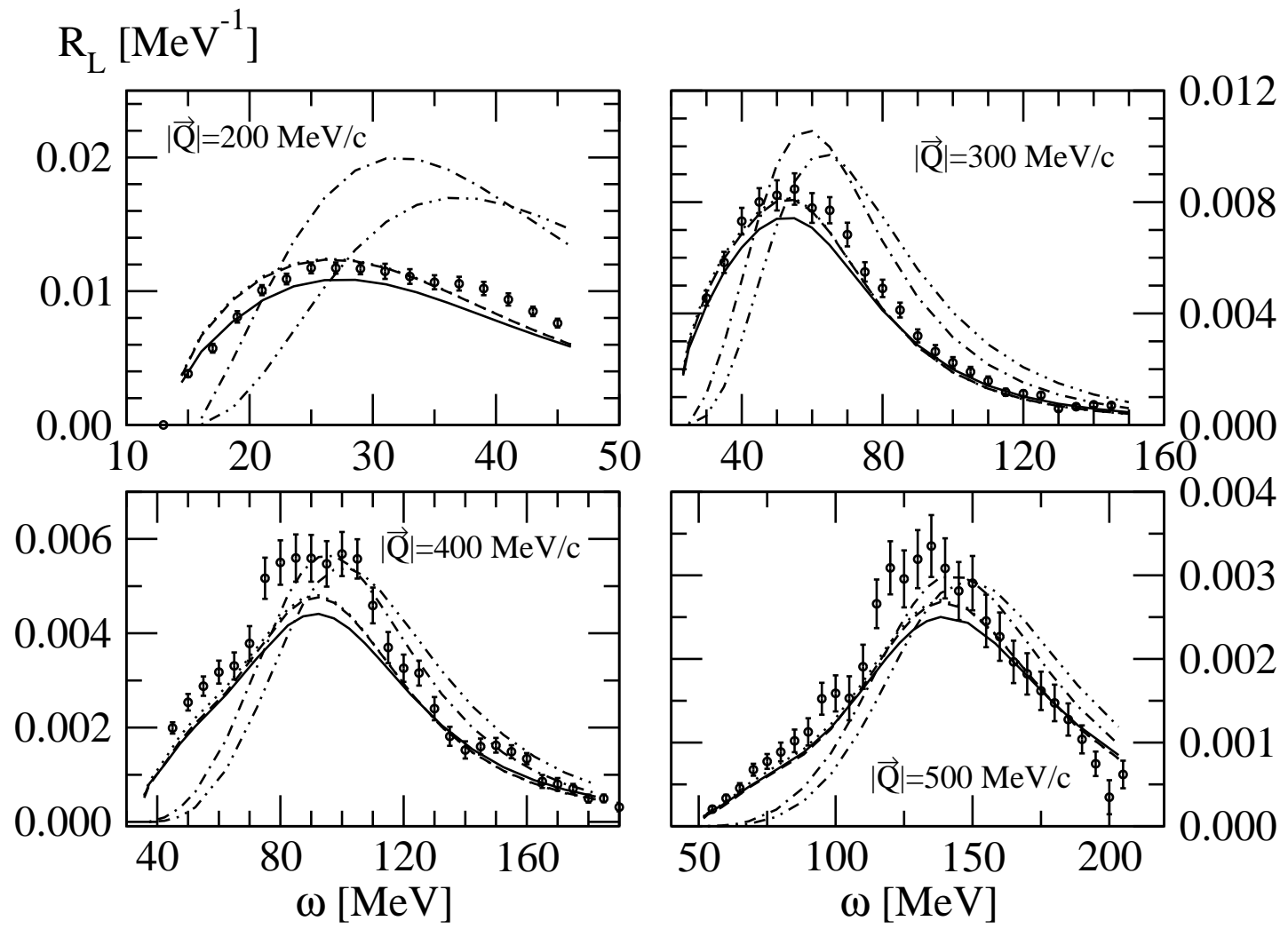

FIG. 23: The longitudinal response function of ${ }^{3} \mathrm{H}$ for different magnitudes of the three-momentum transfer. The double-dot-dashed curve represents the prediction based on the extreme PWIA. The dot-dashed curve was obtained under the assumption that FSI acts only in one two-nucleon subsystem (the so-called FSI23), the dotted curve takes the full FSI into account but neglects MEC and $3 \mathrm{NF}$ effects. The $\pi$ - and $\rho$-like two-body densities are accounted for in the dashed curve and finally the full dynamics including MEC and the $3 \mathrm{NF}$ is given by the solid curve. The dotted and dashed curves practically overlap. Data are from [127].

away from the data. In the case of $R_{L} 3 \mathrm{NF}$ as well as MEC effects turn out to be small, except at $|\vec{Q}|=174 \mathrm{MeV} / \mathrm{c}$ where $3 \mathrm{NF}$ effects for ${ }^{3} \mathrm{He}$ shift theory downwards in direction to the data. For $R_{T}$ MEC effects are again quite significant, shifting the theory upwards. The counteractive effect of the $3 \mathrm{NF}$ is only seen at $|\vec{Q}|=174 \mathrm{MeV} / \mathrm{c}$.

For a smaller $\omega$ region (not explicitly displayed) there are also results for $R_{L}$ of the Trento group [130]. They use the same dynamical input but without two-body densities. Taking that into account the agreement between ours and the Trento group results in the case of 

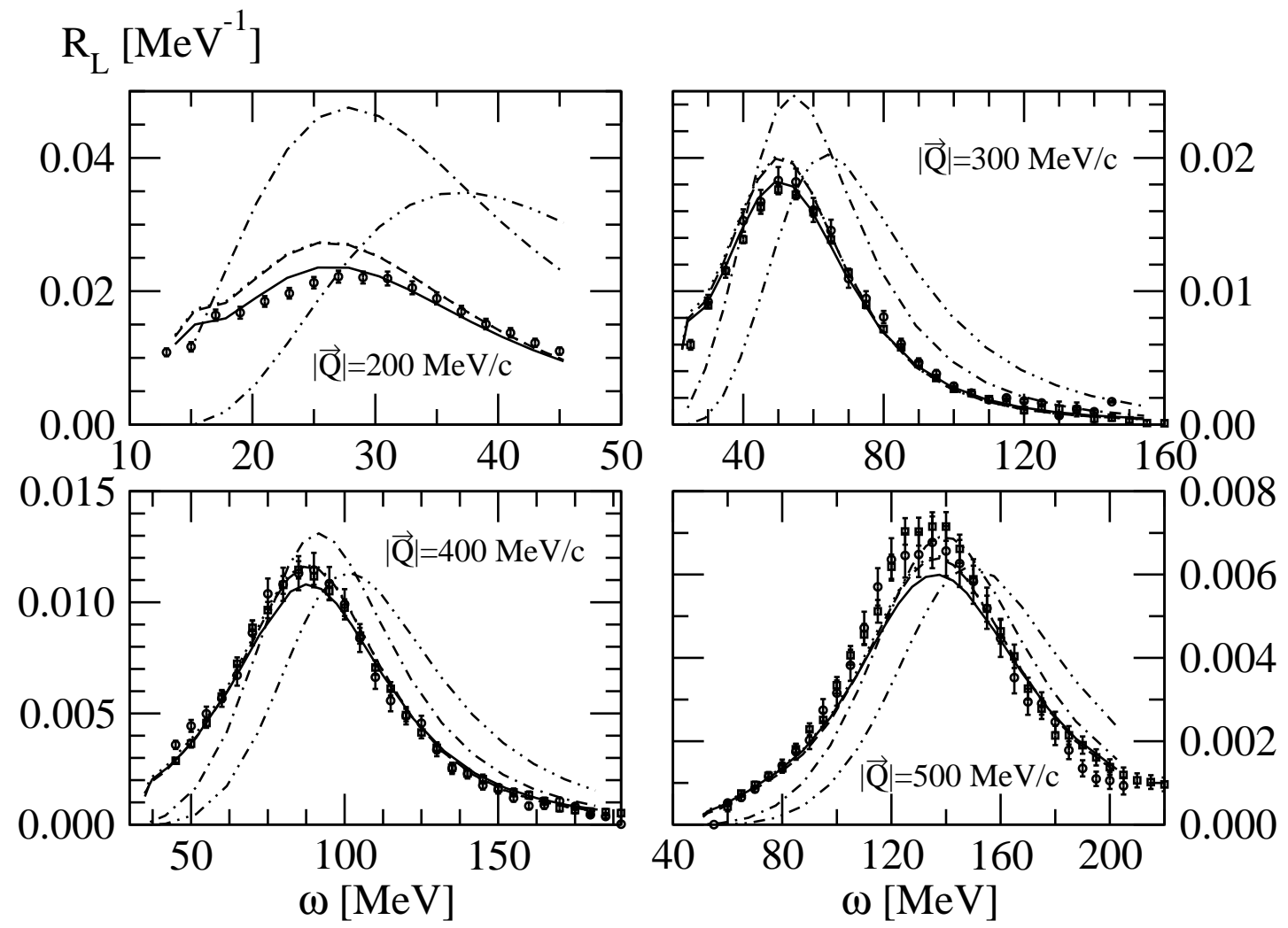

FIG. 24: The same as in Fig. 23 for ${ }^{3}$ He. Data are from [127] (circles) and from [128] (squares).

${ }^{3} \mathrm{H}$ is good. In case of ${ }^{3} \mathrm{He}$ a quantitative comparison is not possible, since we do not include the Coulomb force in the continuum. Going to the $\omega$-region below the three-body breakup in case of ${ }^{3} \mathrm{He}$ one can compare to the results from the Pisa group [59]. Despite the fact that we do not include the Coulomb force in continuum we find in the case of $R_{L}$ a similar increase of the two-body density effect with increasing $|\vec{Q}|$-values, namely a shift downwards. In the case of $R_{T}$ we find a similar, upwards shift of the two-body current effects. Our curves including FSI+MEC+3NF lie a bit higher in comparison to the ones in [59] but still rather close to the data.

We would like to present another set of data for threshold electrodisintegration of ${ }^{3} \mathrm{He}$ [131], where the electron scattering angle was $160^{\circ}$, emphasizing the contribution from $\mathrm{R}_{T}$. The cross section is shown in Fig. 31. Again the absolute need for interaction in the continuum is obvious, but furthermore also significant effects of MEC and 3NF are visible. The agreement of our theory with the data is very good. Further data displayed in [131] require relativity and are therefore not shown here (see, however, [67], where some selected 

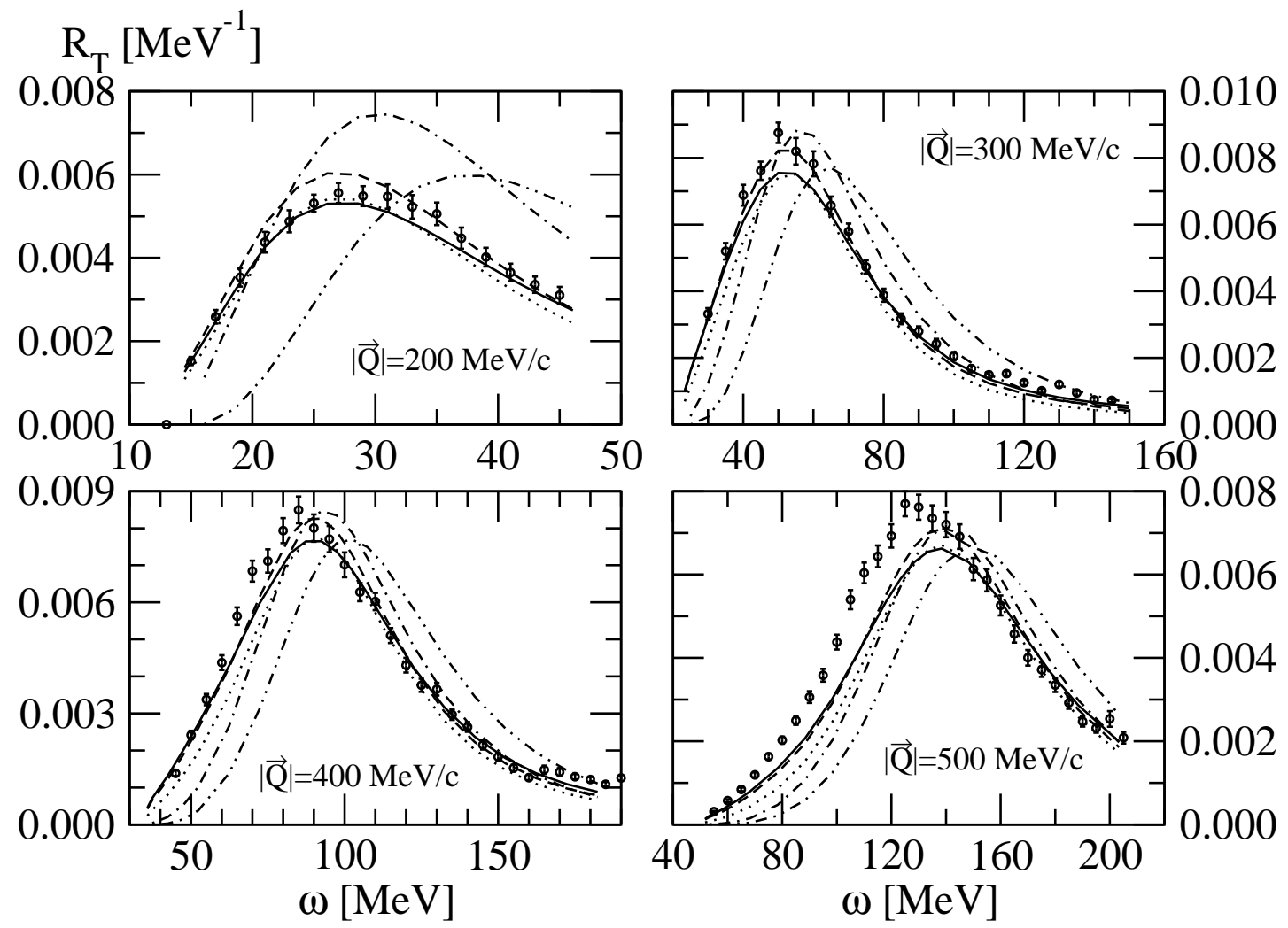

FIG. 25: The transverse response functions $R_{T}$ for ${ }^{3} \mathrm{H}$. Curves as in Fig. 23, except that the two-body density is replaced by the two-body current. Data are from [127].

relativistic corrections shift theory in the direction to the data).

Finally, Fig. 32 displays $180^{\circ}$ inelastic electron scattering cross sections for ${ }^{3} \mathrm{He}$ at rather low incident electron energies [132]. We see three theoretical curves, one with AV18 alone in the continuum (the dotted curve), then using AV18 alone + MEC (the dashed curve) and finally our most complete calculation with AV18+MEC+3NF (the solid curve). There are strong up and down effects against the pure NN force predictions adding MEC and the $3 \mathrm{NF}$. Though our most complete theoretical prediction is close to the data at the strong rise for the lowest excitation energies, it clearly underpredicts the data at the higher excitation energies.

Previous calculations for the inclusive responses aside from the pioneering one [36] mentioned in the introduction, appeared in [59, 130, 133]. In [133] the longitudinal response was determined with the LIT method combined with a Faddeev decomposition and carried 

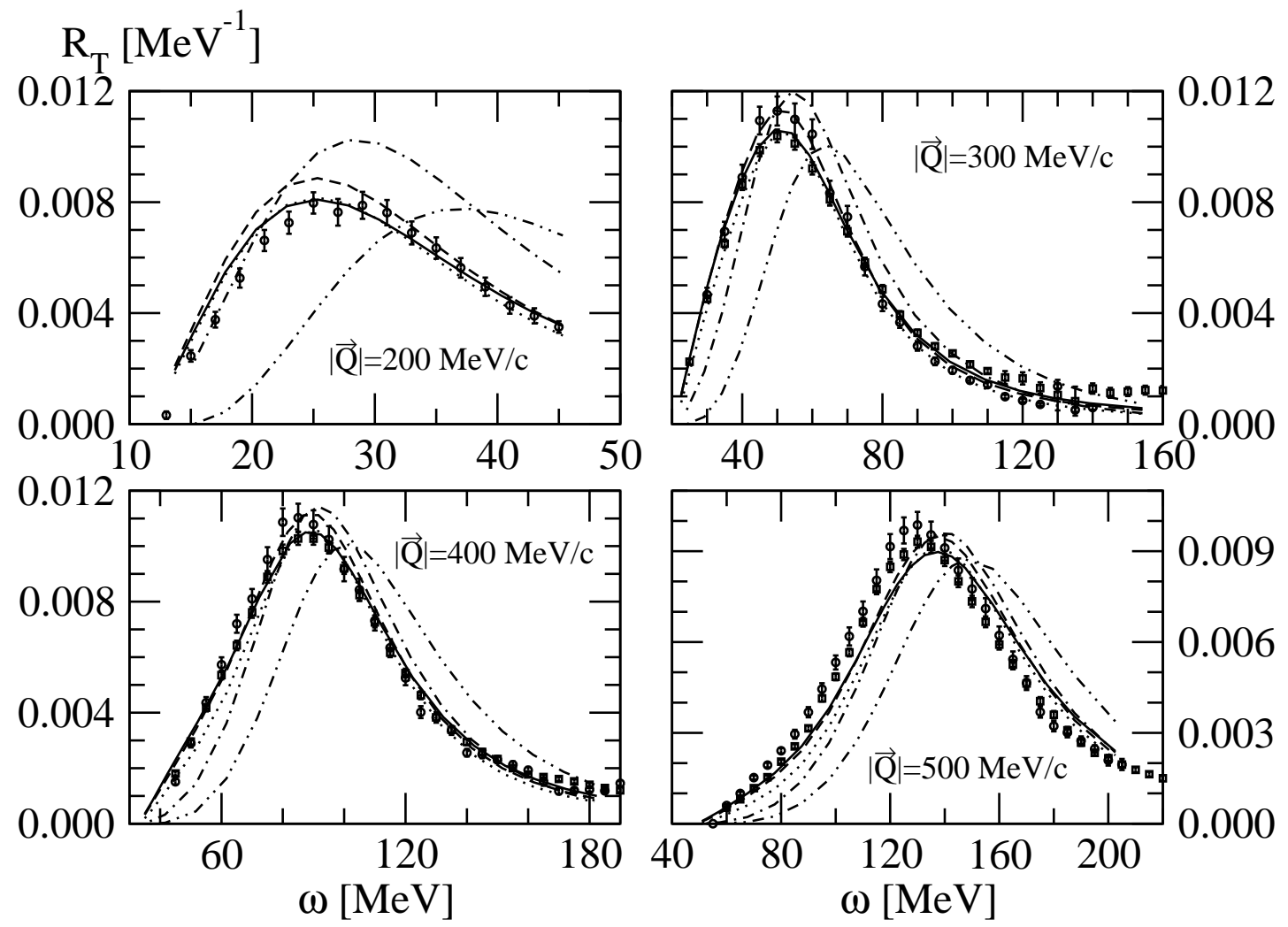

FIG. 26: The same as in Fig. 25 for ${ }^{3}$ He. Data are from [127] (circles) and from [128] (squares).

through in momentum space. A qualitative agreement with experimental data was achieved using the Bonn B [134] NN interaction and the nonrelativistic single nucleon density operator. In [130] the longitudinal response functions were determined via the LIT method using correlated sums of products of hyperspherical functions, hyperspherical harmonics, and spin-isospin factors. The configuration space Bonn A [134] and AV18 NN potentials including the UrbanaIX and Tucson-Melbourne [135] 3NF's were used and standard relativistic corrections of lowest order for the density operator were included. Quite remarkable is the fact that, because the LIT method requires only bound state-like solutions, it was possible to include the Coulomb force also in the final state.The results for $|Q|$-values up to $500 \mathrm{MeV} / \mathrm{c}$ are quite similar to the ones shown above. We mention the decrease of the peak heights adding a $3 \mathrm{NF}$ and the different effects on ${ }^{3} \mathrm{H}$ and ${ }^{3} \mathrm{He}$, namely an underestimation for ${ }^{3} \mathrm{H}$ and a reasonable agreement for ${ }^{3} \mathrm{He}$. Also the $R_{L}$ results in [130] for the ${ }^{3} \mathrm{H}$ data in [129] agree quite well with ours shown in Fig. 27 except for $|Q|=487 \mathrm{MeV} / \mathrm{c}$, where in [130] an overestimation is visible. The same data of [129] were also analyzed in [59], now for the 


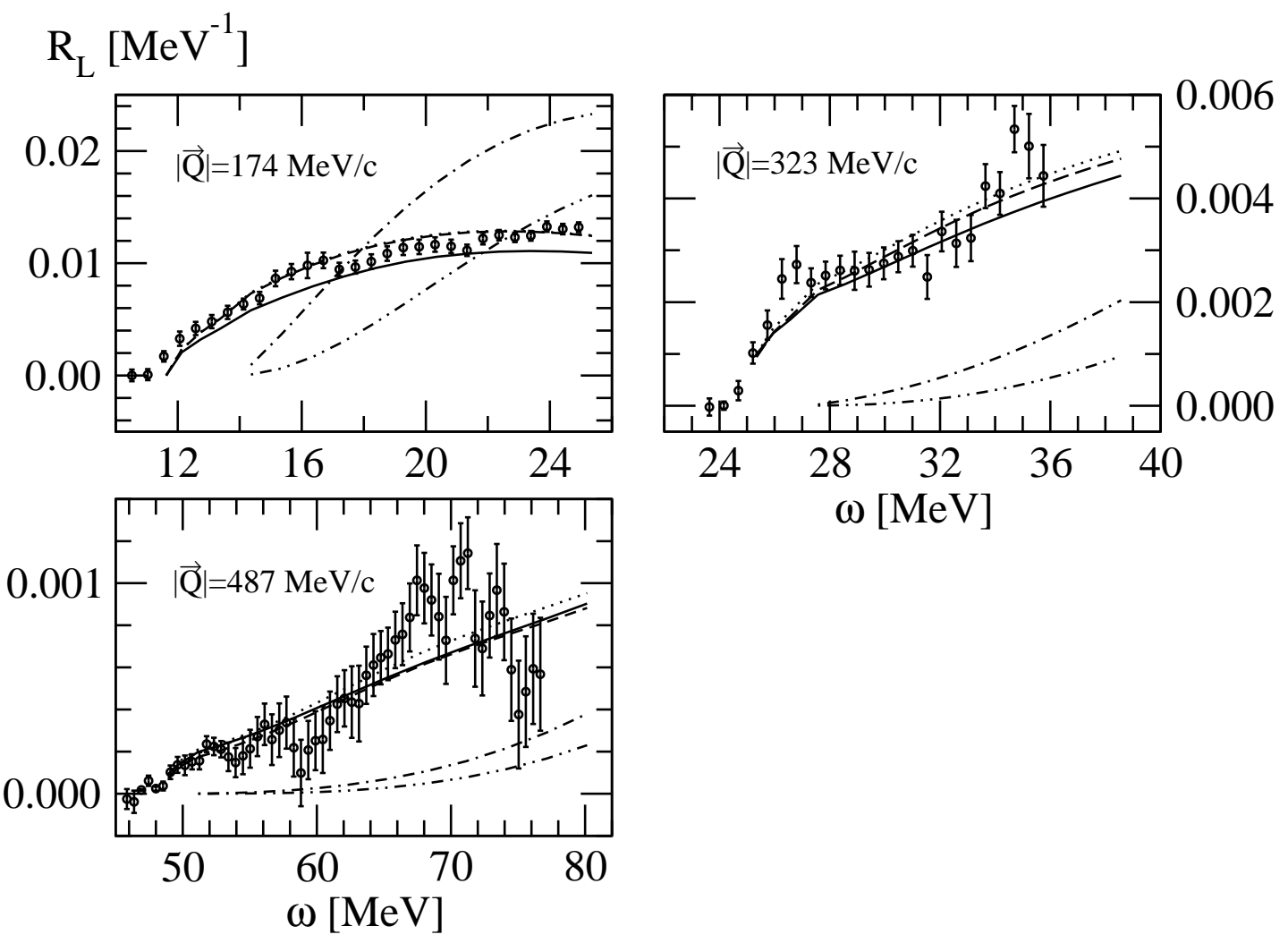

FIG. 27: The longitudinal response function $R_{L}$ for ${ }^{3}$ H. Curves as in Fig. 23. Data are from [129].

longitudinal as well as the transversal responses, but staying below the three-body breakup. The ${ }^{3} \mathrm{He}$ and pd scattering state wave functions were obtained variationally with the pair correlated hyperspherical harmonics method. Again the AV18 and AV18+UrbanaIX were used and the Coulomb force was also fully included. The currents and densities are as described above, but additional pieces are added which are not constrained by the current conservation. This also includes terms related to the $\Delta$-excitation. The agreement to the data is comparable to the one shown above. Below the three-body threshold the results for the longitudinal response for ${ }^{3} \mathrm{He}$ agree well with the ones in [130] at the two larger $|Q|$-values.

Finally the Euclidean longitudinal and transversal responses have been worked out . As a Laplace transform in the energy transfer $\omega$ the response is mapped onto an imaginary time $\tau$. The technically very attractive feature is that the Euclidean response can be cast in a pathintegral form which can be naturally evaluated with Monte Carlo techniques. We refer the reader to the original literature $[26,136-138]$ for the interesting insights into the propagation 


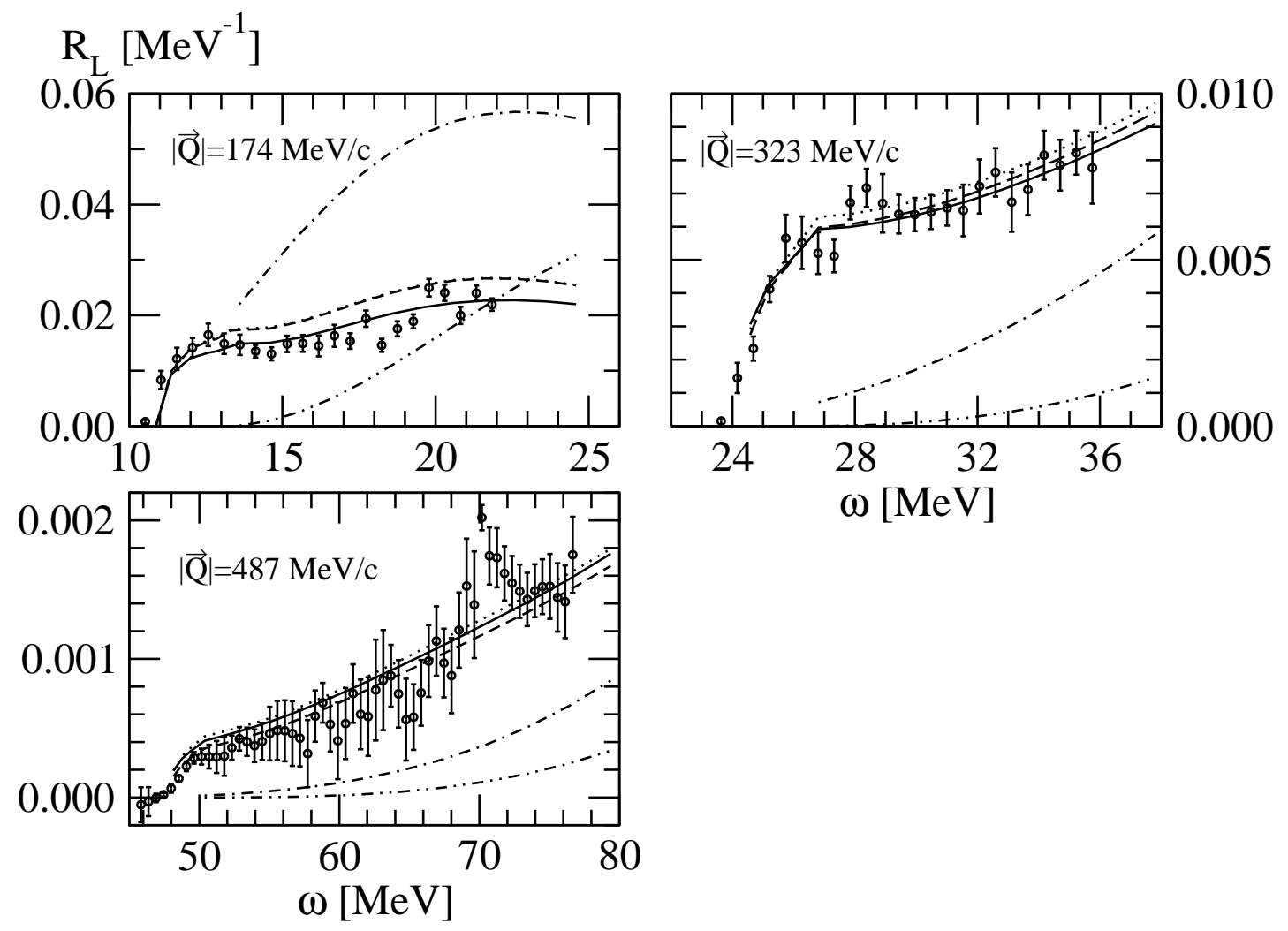

FIG. 28: The same as in Fig. 27 for ${ }^{3}$ He. Data are from [129].

of charge with increasing $\tau$ and the comparison with correspondingly transformed data. In [139] a thorough study on both (longitudinal and transversal) Euclidean response functions have been performed and compared to the world data. This also includes ${ }^{4} \mathrm{He}$, which sheds light on the access to the transverse quasi-elastic strengths. Also sum rules techniques were employed to study the $\mathrm{T} / \mathrm{L}$ ratios. Nevertheless the Laplace transform of the data looses details and appears not to be a substitute of evaluating the responses directly for fixed $|Q|$.

In addition, data are available for the cases, where both initial particles, the electron and ${ }^{3} \mathrm{He}$, are polarized. This allows to access two more response functions, $R_{T^{\prime}}$ and $R_{T L^{\prime}}$. Data for $R_{T^{\prime}}$ and $R_{T L^{\prime}}$ alone are not yet taken to the best of our knowledge, only asymmetries. In PWIA $R_{T^{\prime}} \propto\left(G_{M}^{n}\right)^{2}$ [140]. Thus measurements concentrated on the transversal asymmetry $A_{T^{\prime}}\left(\theta^{\star} \approx 0^{\circ}\right)$ what according to $(201)$ focuses on $R_{T^{\prime}}$. That sensitivity to the magnetic form factor of the neutron survives despite the fact that PWIA is insufficient [141, 142]. This is documented in Fig. 33 for $A_{T /}$. We show three groups of curves where within each group $G_{M}^{n}$ is multiplied by the factors $0.9,1.0$ and 1.1 . The sensitivities to changes of $G_{M}^{n}$ values 


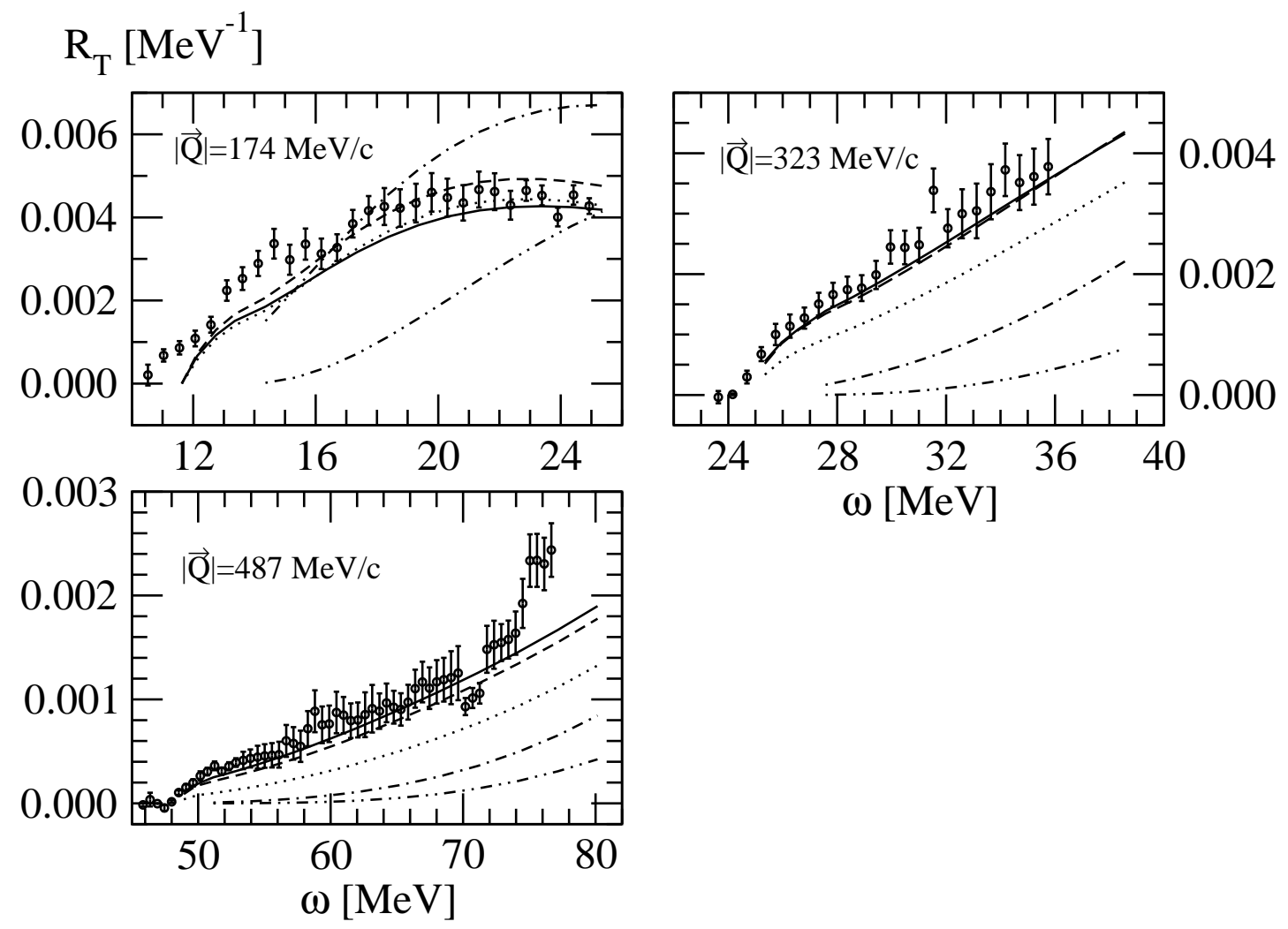

FIG. 29: The transverse response function $R_{T}$ for ${ }^{3} \mathrm{H}$. Curves as in Fig. 23. Data are from [129].

are very similar whether one uses PWIA, FSI23 or our complete picture FSI+MEC+3NF. Therefore the measurement of $A_{T}$, for polarized ${ }^{3} \mathrm{He}$ is a good tool to extract $G_{M}^{n}$ because we can consider $G_{M}^{n}$ the only unknown dynamical input for our calculations. The dependence of $A_{T^{\prime}}$ on the electric form factor of the neutron $G_{E}^{n}$, which still has rather big error bars, is negligible. Therefore one can use the measured values of $A_{T^{\prime}}$ and adjust $G_{M}^{n}$. For the detailed procedure we refer to $[141,142]$. The theoretical results against the data are displayed in Fig. 34 for $Q^{2}=0.1$ and $0.2(\mathrm{GeV} / \mathrm{c})^{2}$. While PWIA has the wrong slope, already the inclusion of the NN interaction in the spectator pair (FSI23) leads to the correct shape, though it lies high above the data. Complete FSI is important and the NN force prediction alone comes rather close to the data. On top we show the MEC effects which are quite noticeable and the somewhat smaller 3NF effects. The latter ones lower the theoretical prediction on top of the shift caused by MEC. A direct comparison of our new results to the ones presented in $[141,142]$ reveals some differences. The reasons for those differences are manifold. In [142] we did not use AV18 plus the explicit $\pi$ - and $\rho$-like two-body currents 

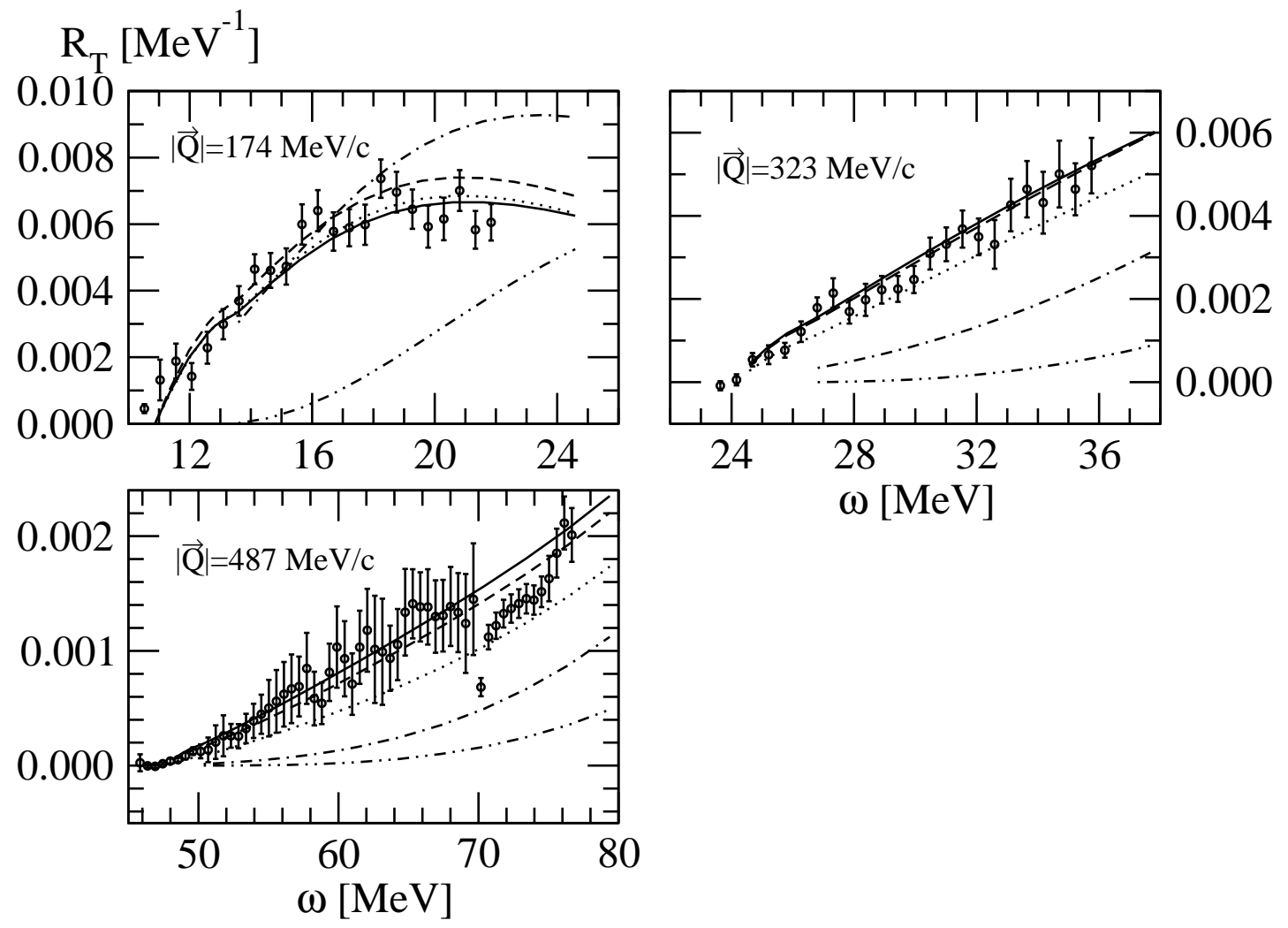

FIG. 30: The same as in Fig. 29 for ${ }^{3}$ He. Data are from [129].

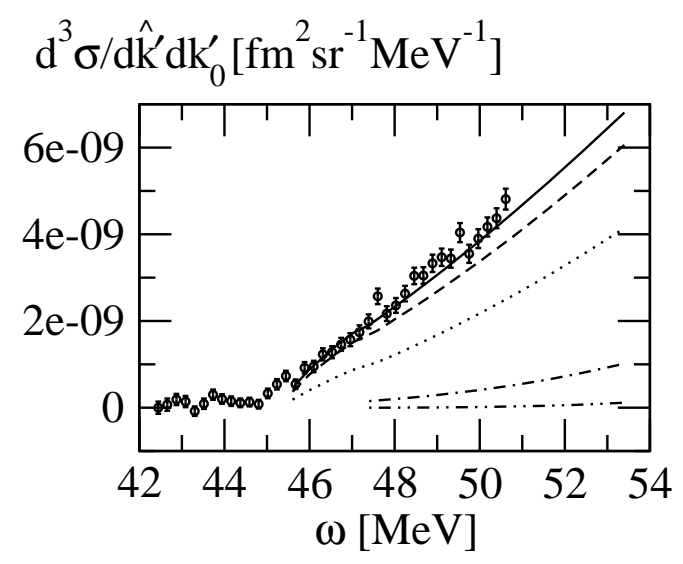

FIG. 31: The inclusive differential cross section $\frac{d^{3} \sigma}{d \hat{k}^{\prime} d k_{0}^{\prime}}$ taken at $\vartheta=160^{\circ}$ as a function of the energy transfer $\omega$ for the electron beam energy of $263 \mathrm{MeV}$. Curves as in Fig. 23. Data are from [131].

but Bonn B with the standard $\pi$ - and $\rho$-currents augmented by the strong form factors of 

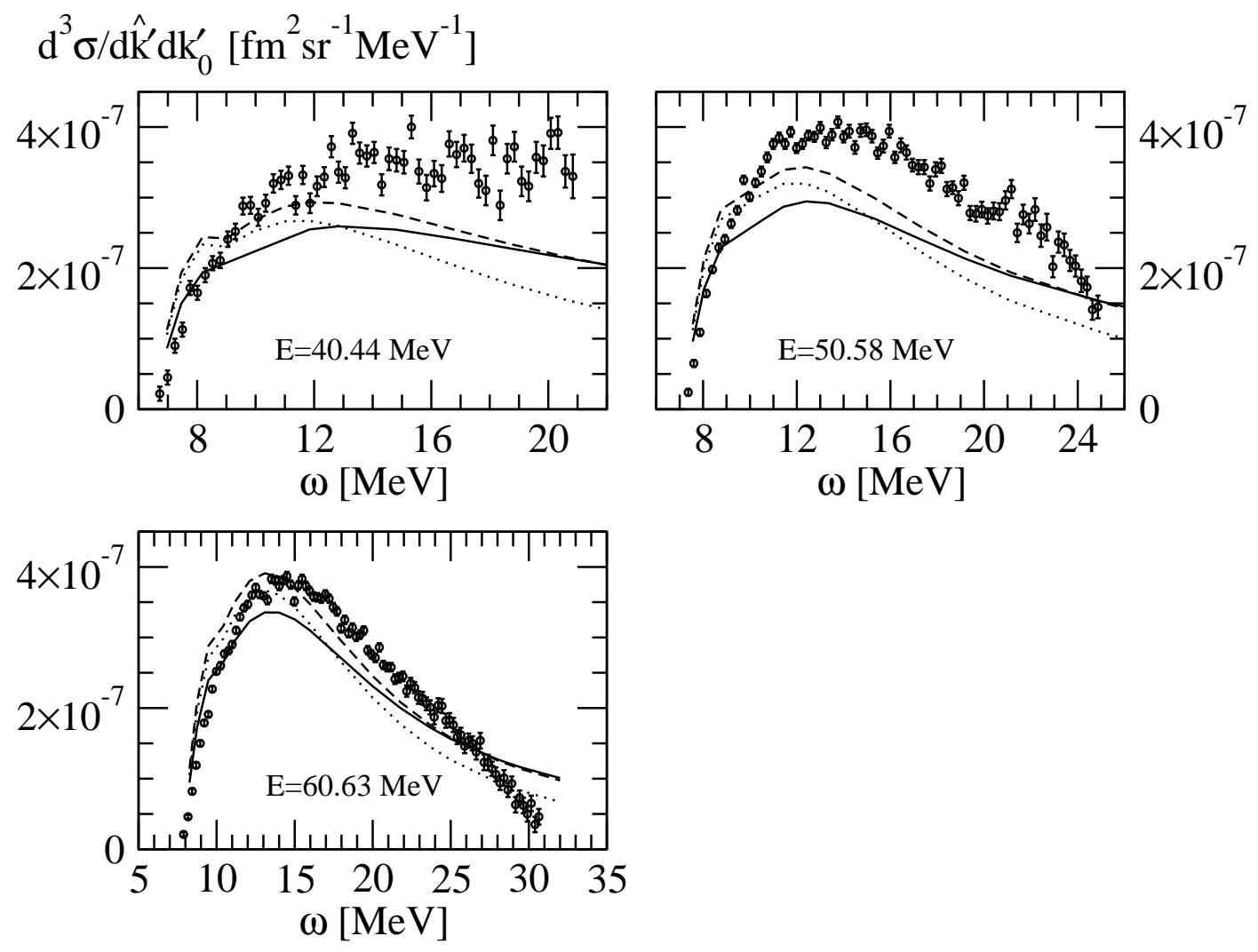

FIG. 32: The inclusive differential cross section $\frac{d^{3} \sigma}{d \hat{k}^{\prime} d k_{0}^{\prime}}$ taken at $\vartheta=180^{\circ}$ as a function of the energy transfer $\omega$ for three electron beam energies. For the description of the curves see Fig. 23. Data are from [132].

Bonn B. In addition we use now $G_{E}^{p}$ in the charge density and $G_{E}^{p}$ and $G_{E}^{n}$ in the convection current, while in the previous works we employed $F_{1}^{p}$ and $F_{1}^{n}$. We also replaced the Höhler models for the electromagnetic form factors [143] by the electromagnetic form factors [83] based on a dispersion theoretical analysis. Further now we also add the two-body density.

At this point we would like to add a more conceptual remark. In the spirit of a Hamiltonian approach the arguments of the nucleon form factors are the difference of the fourmomenta of the nucleons squared, before and after the photon absorption, and not the four-momentum squared of the photon, which would be required in a manifestly covariant formalism. The reason is that in a Hamiltonian formalism, where the nucleons are on the mass-shell one has only three-momentum conservation at the photon vertex. Then since we nearly always neglect relativistic features we choose as arguments of the electromagnetic 
form factors just $(\vec{Q})^{2}$. In the case of real photons and in our momentum region, $(\vec{Q})^{2}$ is very small and we put it simply to zero.

In addition to all that we allow now for np and pp (nn) forces using the " $\frac{2}{3}-\frac{1}{3}$ " rule, while in the previous work $[141,142]$ we used np forces only. Finally the deuteron and the ${ }^{3} \mathrm{He}$ wave functions are generated with all the electromagnetic pieces of the AV18 interaction and thus especially the pp Coulomb force as the dominant part is now taken into account in ${ }^{3} \mathrm{He}$. Based on all that and noting that in [142] the theory was averaged over the spectrometer acceptances using a Monte Carlo simulation, while in Fig. 34 we show point geometry results, some differences to the previous results had to be expected. Therefore one has to accept that a renewed extraction of $\mathrm{G}_{M}^{n}$ from the data given in [142] would provide a slightly different result. We did not perform that study since we have no more access to the experimental conditions and moreover our theory is anyhow only some intermediate step toward a more basic concept.

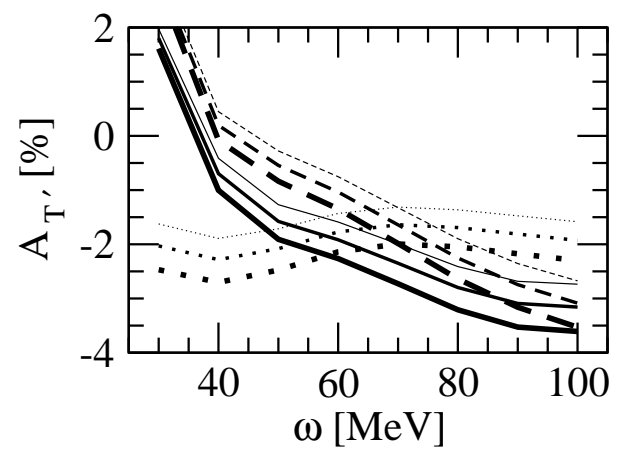

FIG. 33: Sensitivity of the asymmetry $A_{T^{\prime}}$ to the changes of $G_{M}^{n}$ for the four-momentum transfer squared $Q^{2}=0.1(\mathrm{GeV} / \mathrm{c})^{2}$. Three groups of curves with $0.9 G_{M}^{n}, 1.0 G_{M}^{n}$ and $1.1 G_{M}^{n}$ are shown for PWIA (dotted), FSI23 (dashed) and FSI+MEC+3NF (solid). In each case the upper curve is for 0.9 , the middle one for 1.0 and the lower one for 1.1 .

The resulting values for $G_{M}^{n}$ extracted in [142] are shown in Fig. 35 together with the values extracted from the deuteron $[144,145]$. The agreement between the two totally independent approaches is very good, though one should keep the above remarks in mind.

The analysis of the $A_{T^{\prime}}$ data at $Q^{2}=0.3-0.6(\mathrm{GeV} / \mathrm{c})^{2}$ also measured in [142] is outside the present theoretical framework and we refer the reader to [147], where $G_{M}^{n}$-values were extracted under the assumption of a plane wave impulse approximation. This work uses 

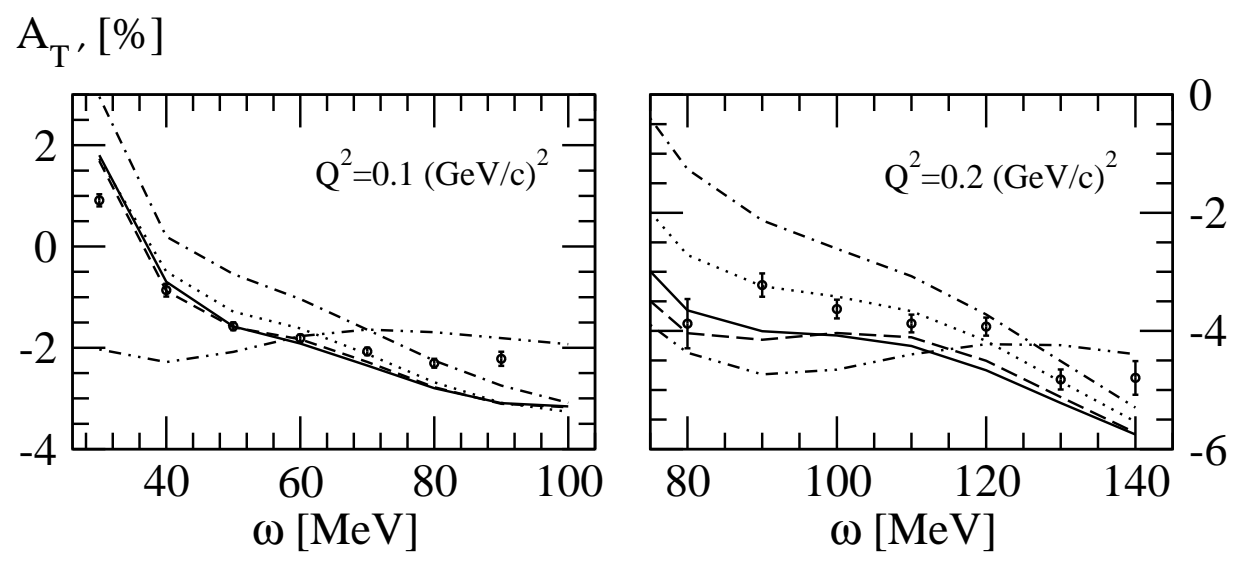

FIG. 34: The asymmetry data from [142] against theory for two different four-momentum transfers squared $Q^{2}=0.1(\mathrm{GeV} / \mathrm{c})^{2}$ (left) and $0.2(\mathrm{GeV} / \mathrm{c})^{2}$ (right). PWIA (double-dot-dashed), FSI23 (dot-dashed), FSI with NN forces alone (dotted), FSI with NN forces alone + MEC (dashed) and adding in the $3 \mathrm{NF}$ (solid).

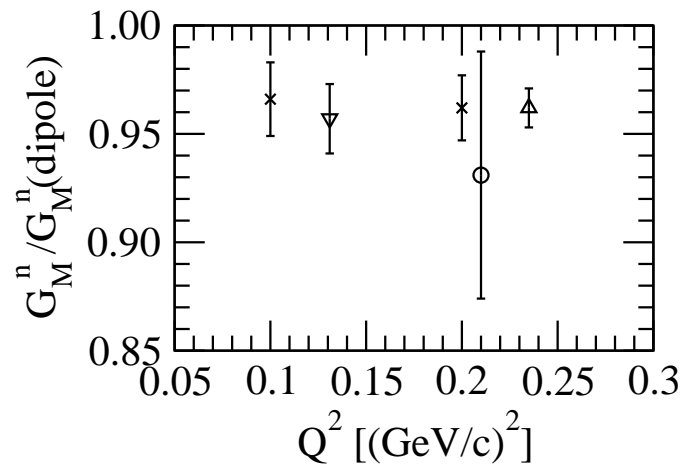

FIG. 35: $G_{M}^{n}$-values extracted from different measurements on the deuteron $([144](\nabla),[145](\triangle))$ and on ${ }^{3} \mathrm{He}([146](\circ),[142](\times))$. For the sake of visibility the two deuteron results $(\nabla$ and $\triangle)$ are shifted sidewards but belong to $Q^{2}=0.1$ and $0.2(\mathrm{GeV} / \mathrm{c})^{2}$, respectively.

the concept of the spin dependent spectral function of the three-body system and employs realistic forces [148]. The polarized responses $R_{T L^{\prime}}$ and $R_{T^{\prime}}$ were evaluated with the aim to minimize the model dependence in the extraction of the neutron electromagnetic form factors. Thereby the prominent role of the proton contributions got illuminated.

The interplay of both response functions $R_{T^{\prime}}$ and $R_{T L^{\prime}}$ in (201) has been investigated in 
[149] by choosing $\theta^{\star}$ in a small range around $135^{\circ}$. The resulting asymmetries $A$ are shown in comparison to our theoretical results for $Q^{2}=0.1$ and $0.2(\mathrm{GeV} / \mathrm{c})^{2}$ in Fig. 36. Again MEC's effects are quite important, and they are slightly modified by the addition of the $3 \mathrm{NF}$. The agreement with the data is quite good.

Finally we want to draw attention to the question whether signatures of short-range NN correlations can be extracted from inclusive responses. A nice general introduction with appropriate references is given in [26], thus we shall not repeat it here. The keypoint is to regard the energy integral over the longitudinal response function (Coulomb sum rule), which can be separated into nucleon form factor parts, the elastic charge form factor of the nucleus, and a third part, which under the simplest assumption is the Fourier transform of the proton-proton correlation function. As nicely shown in [150] that third part is in addition strongly influenced by relativistic corrections and two-body pieces in the density operator. Unfortunately that third part carries large experimental error bars due to the strong cancellations of the Coulomb sum with the first two parts. That third part would be an excellent piece of information on nuclear dynamics if the data base could be improved, especially the high wings of the longitudinal responses. An older investigation of our collaboration can be found in [151].
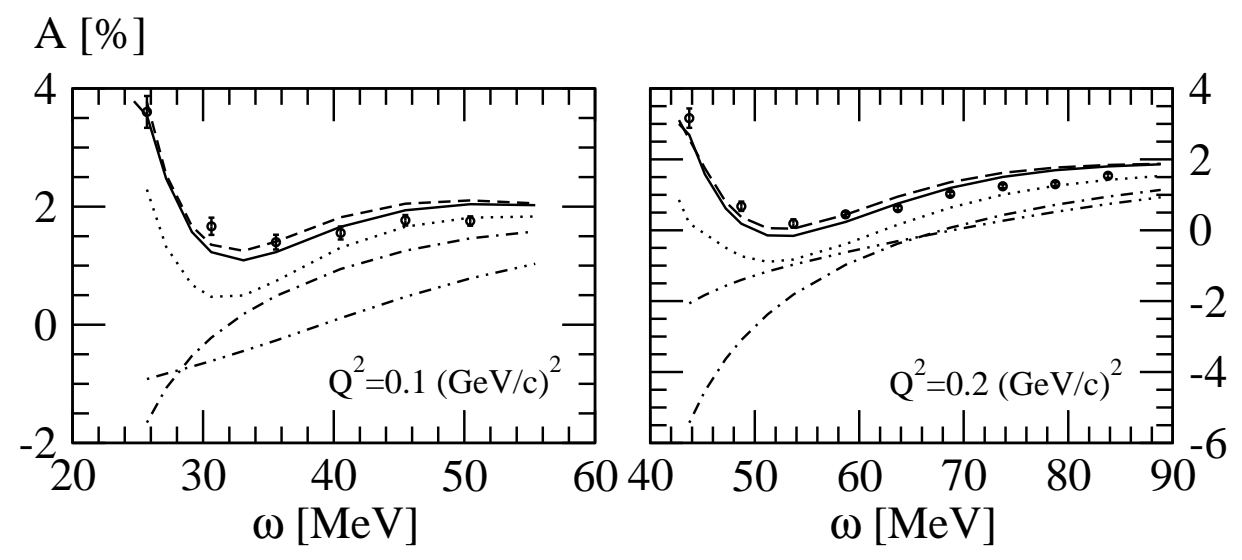

FIG. 36: The asymmetry data from [149] against theory for two different four-momentum transfers squared $Q^{2}=0.1(\mathrm{GeV} / \mathrm{c})^{2}$ (left) and $0.2(\mathrm{GeV} / \mathrm{c})^{2}$ (right). Curves as in Fig. 34 . 


\section{Electron induced pd breakup of ${ }^{3} \mathrm{He}$}

There is a big group of data taken at NIKHEF [152], presented in [153] and communicated to us by E. Jans [154]. The different kinematical conditions named as in [153] are shown in Table II. The proton and deuteron momenta lie in the plane spanned by the electron momenta. For the configurations T1, T2 and C1 data were taken for proton scattering angles close to the photon direction, while for $\mathrm{C} 2$ and $\mathrm{C} 3$ the proton directions are further off. The quasi free scattering condition $Q_{0}=\frac{\vec{Q}^{2}}{2 m_{N}}$ is not covered by the data. We show in Figs. 37-41 the angular distributions of the proton against the electron beam direction for those five configurations. Since one is close to the quasi free nucleon knockout peak the photon is absorbed mostly by one nucleon and in plane wave impulse approximation the antisymmetrization plays no role, in other words the PWIA result is very close to the PWIAS result. Also in all cases except C1 PWIA is totally insufficient. The MEC effects are insignificant. For the similar kinematics T1 and C2 the 3NF effects are quite strong and together with the NN force move the theory quite close into the data. Going to higher energy transfers the situation changes and the $3 \mathrm{NF}$ effects are unimportant. This is seen for the kinematics C3 and T2. Finally, in the case of C1, with a relatively small energy transfer and for the high three momentum transfer like in the other cases the FSI, MEC and 3NF effects are all small and all curves overshoot the data somewhat.

\begin{tabular}{ccccc}
\hline & $k_{0}$ & $\theta_{e}$ & $\omega$ & $Q$ \\
& $(\mathrm{MeV})$ & $(\mathrm{deg})$ & $(\mathrm{MeV})$ & $(\mathrm{MeV} / \mathrm{c})$ \\
\hline $\mathrm{T} 1$ & 367.1 & 85.0 & 107.1 & 431.0 \\
$\mathrm{~T} 2$ & 367.1 & 85.0 & 143.8 & 412.7 \\
$\mathrm{C} 1$ & 390.0 & 74.4 & 66.1 & 434.8 \\
$\mathrm{C} 2$ & 390.0 & 79.0 & 110.4 & 434.4 \\
$\mathrm{C} 3$ & 390.0 & 83.0 & 145.1 & 434.5 \\
$\mathrm{HR}$ & 390.0 & 39.7 & 113.0 & 250.2 \\
\hline
\end{tabular}

TABLE II: The NIKHEF electron kinematics specified by different kinematical quantities.

Another set of data under the $H R$ kinematics from Table II is shown in Fig. 42. The data are on the slopes of the proton and deuteron knockout peaks. The deuteron knockout peak 


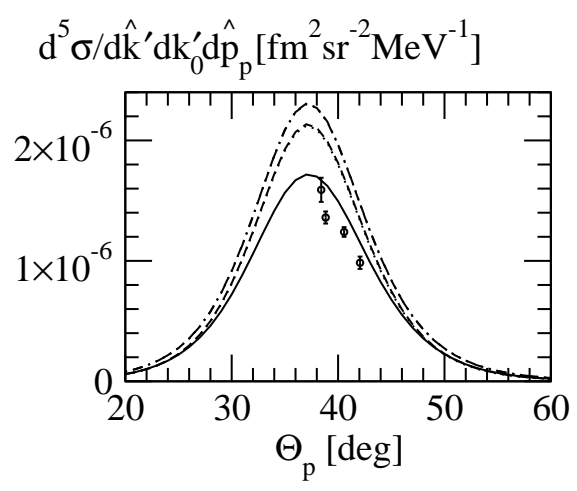

FIG. 37: Proton angular distribution for the T1 configuration from Table II. The double-dotdashed curve represents the prediction based on PWIA. The dot-dashed curve is obtained under the assumption of PWIAS (which overlaps with PWIA), the dotted curve takes the full FSI into account but neglects MEC and 3NF effects. The $\pi$ - and $\rho$-like two-body densities are accounted for additionally in the dashed curve (which overlaps with FSI), and finally, the full dynamics including MEC and the $3 \mathrm{NF}$ is given by the solid curve. Data are from [153].

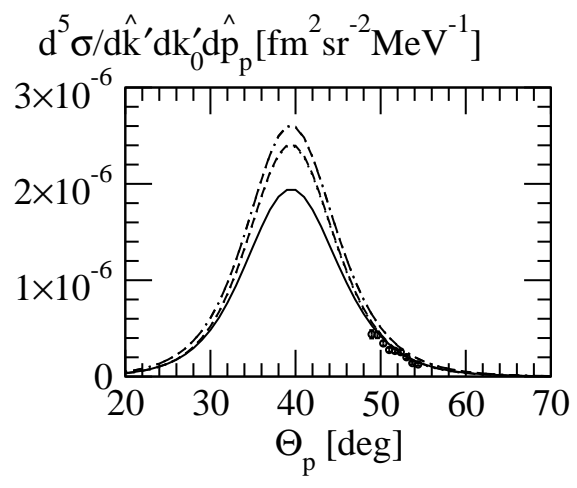

FIG. 38: Proton angular distribution for the C2 configuration from Table II. Curves as in Fig. 37. Data are from [153].

lies around $\theta_{p}=240^{\circ}$. The figure shows nicely how in plane wave impulse approximation the symmetrized version PWIAS deviates around $90^{\circ}$ from the unsymmetrized version PWIA and the absorption of the photon by the other two nucleons takes over and leads to a second peak, the deuteron knockout peak. But the nuclear force effects in the final continuum are extremely important there and shift theory downwards by about one order of magnitude. Also in the slope of the proton knockout peak the final state interactions in the continuum 


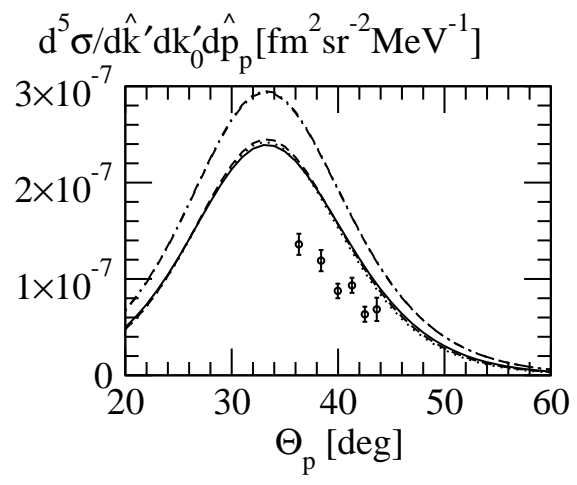

FIG. 39: Proton angular distribution for the T2 configuration from Table II. Curves as in Fig. 37. Data are from [153].

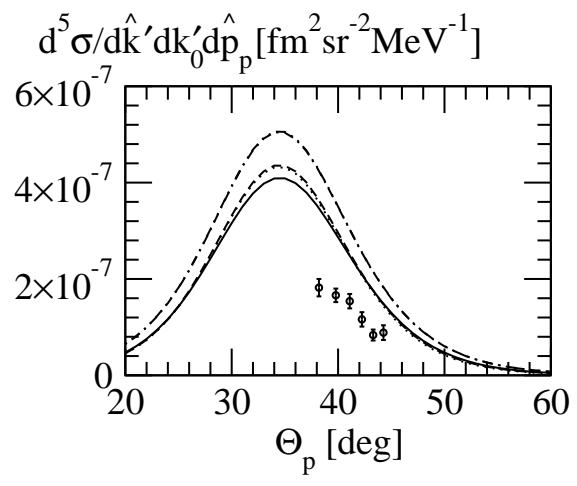

FIG. 40: Proton angular distribution for the C3 configuration from Table II. Curves as in Fig. 37. Data are from [153].

are important. In both cases the agreement with the data is quite good.

Now we concentrate on the deuteron knockout peak and compare the data from [155] and the theory in Figs. 43-47. The PWIA result is extremely small and not displayed. In all cases shown the $3 \mathrm{NF}$ effects on top of the $\mathrm{NN}$ force contributions in the continuum are quite important and move theory close to the data. Note that for $\omega=50 \mathrm{MeV},|\vec{Q}|=412$ $\mathrm{MeV} / \mathrm{c}$ and $\omega=70 \mathrm{MeV},|\vec{Q}|=504 \mathrm{MeV} / \mathrm{c}$ the nuclear matrix elements are similar but the electron kinematics are quite different, which weights the different response functions differently. Thus in the case of $\omega=70 \mathrm{MeV},|\vec{Q}|=504 \mathrm{MeV} / \mathrm{c}$ the MEC effects are significant, while in the other case they are insignificant. In all deuteron knockout peaks the theory clearly overestimates the data. Thus a renewed measurement concentrating on the 


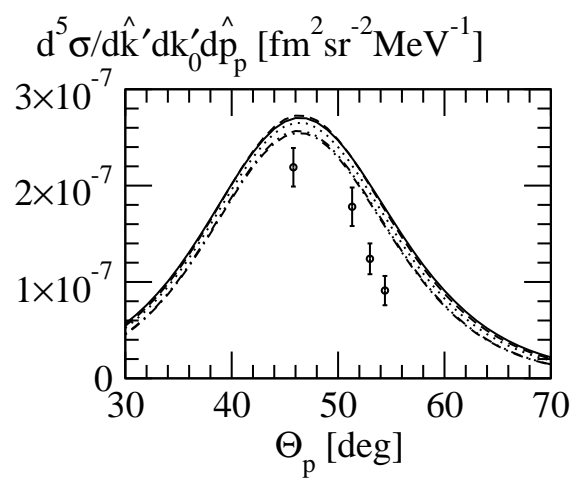

FIG. 41: Proton angular distribution for the C1 configuration from Table II. Curves as in Fig. 37. Data are from [153].

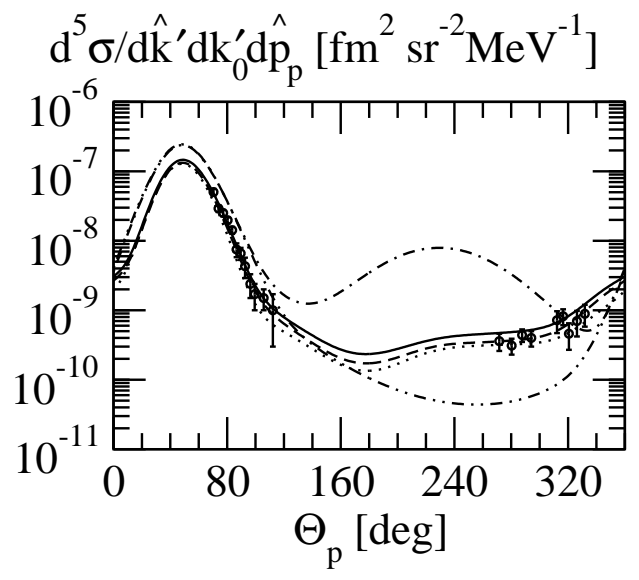

FIG. 42: Proton angular distribution for the HR configuration from Table II. Curves as in Fig. 37. Data are from [153].

missing momentum $p_{m}=0$ would be very desirable.

Further kinematical configurations in search for the deuteron knockout are related to the "D-kinematics" in [153]. In this case the direction of the deuteron has been chosen parallel to the photon direction and the data were taken for $k_{0}=390 \mathrm{MeV},|\vec{Q}|=380 \mathrm{MeV} / \mathrm{c}$ and are displayed in Fig. 48 as a function of the relative kinetic energy $T_{p d}$ of the proton and the deuteron. The agreement is quite good and the effects of the nucleon interactions in the continuum are decisive. None of those data points correspond exactly to the quasi free peak position, where we experienced the discrepancies in Figs. 43-47.

Another set of data [156] in parallel deuteron knockout kinematics is compared to our 


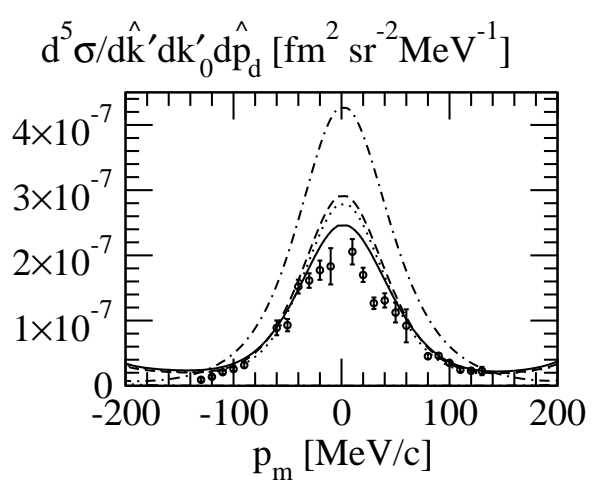

FIG. 43: Deuteron knockout cross section as a function of the missing (i.e. proton) momentum $p_{m}$ for the following electron kinematics: $k_{0}=370 \mathrm{MeV}, \omega=50 \mathrm{MeV},|\vec{Q}|=412 \mathrm{MeV} / \mathrm{c}$. PWIAS (dot-dashed line), FSI (dotted line), FSI+MEC (dashed line) and FSI+MEC+3NF (solid line) results are compared to experimental data from [155]. Note that the PWIA result is very small and therefore not displayed.

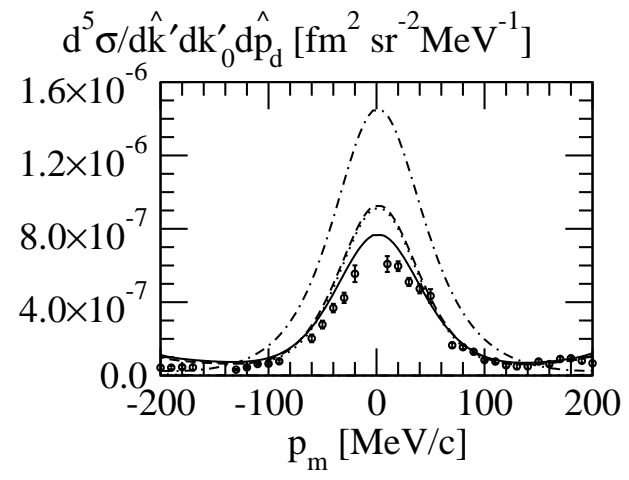

FIG. 44: The same as in Fig. 43 for $k_{0}=576 \mathrm{MeV}, \omega=50 \mathrm{MeV},|\vec{Q}|=412 \mathrm{MeV} / \mathrm{c}$. Data are from $[155]$.

theory in Figs. 49-53. Data were taken at three different $|\vec{Q}|$-values $(|\vec{Q}|=412,504$ and $604 \mathrm{MeV} / \mathrm{c}$ ) and at two electron beam energies $\left(E_{e}=370\right.$ and $\left.576 \mathrm{MeV}\right)$. In all cases FSI is quite important, whereas the addition of MEC's and/or 3NF's yields only marginal shifts, at least in the range of $p_{m}$ values, which were covered by the data. The agreement with the data is reasonably good.

Recently these data have been reanalyzed in [65] including a single $\Delta$-isobar excitation. The results, agreements and disagreements, are very similar to ours shown in Figs. 37-53. 


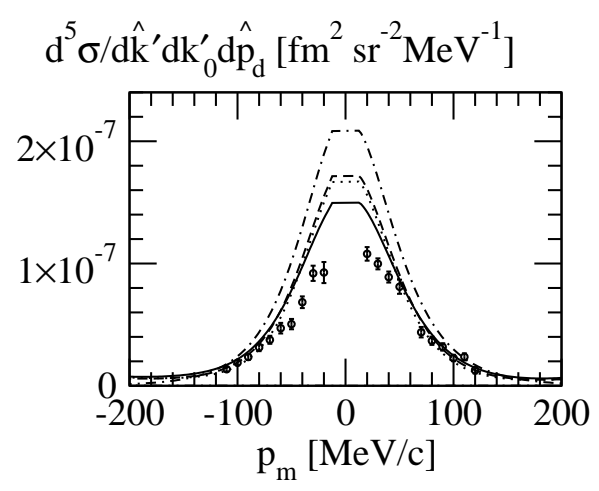

FIG. 45: The same as in Fig. 43 for $k_{0}=576 \mathrm{MeV}, \omega=70 \mathrm{MeV},|\vec{Q}|=504 \mathrm{MeV} / \mathrm{c}$. Data are from $[155]$.

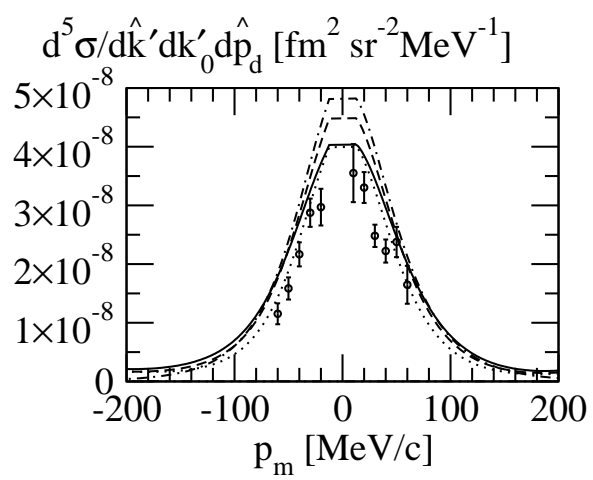

FIG. 46: The same as in Fig. 43 for $k_{0}=370 \mathrm{MeV}, \omega=70 \mathrm{MeV},|\vec{Q}|=504 \mathrm{MeV} / \mathrm{c}$. Data are from [155].

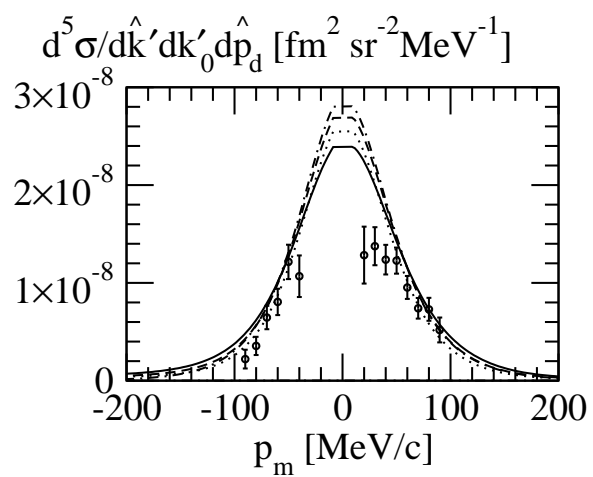

FIG. 47: The same as in Fig. 43 for $k_{0}=576 \mathrm{MeV}, \omega=100 \mathrm{MeV},|\vec{Q}|=604 \mathrm{MeV} / \mathrm{c}$. Data are from [155]. 


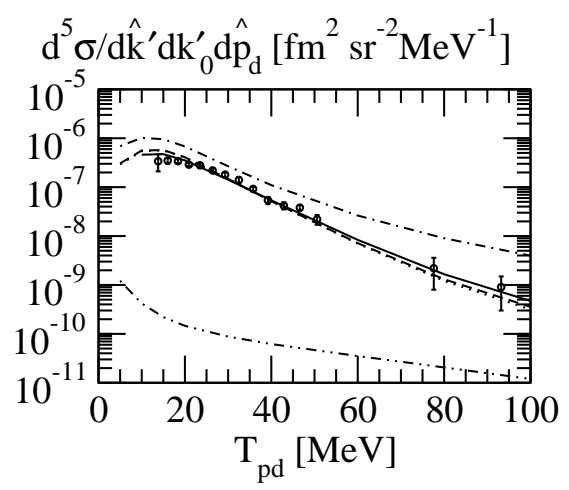

FIG. 48: Deuteron knockout cross section as a function of the relative proton-deuteron energy $T_{p d}$ for the parallel kinematics with $E_{e}=390 \mathrm{MeV}$ and $|\vec{Q}|=380 \mathrm{MeV} / \mathrm{c}$. Curves as in Fig. 43. Data are from [153].

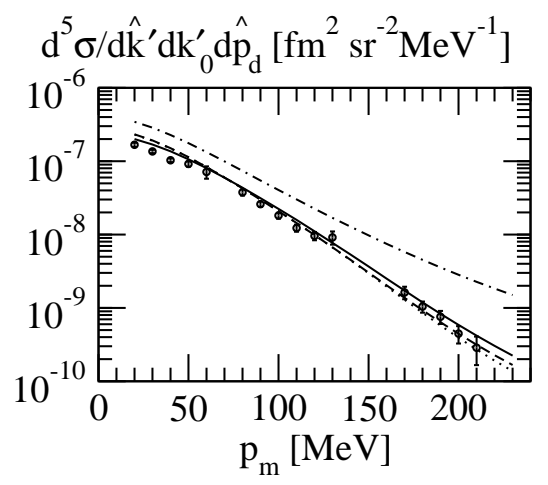

FIG. 49: Deuteron knockout cross section as a function of the missing momentum $p_{m}$ for the parallel kinematics with $E_{e}=370 \mathrm{MeV}$ and $|\vec{Q}|=412 \mathrm{MeV} /$ c. Curves as in Fig. 43 but the PWIA results are not displayed. Data are from [156]. 


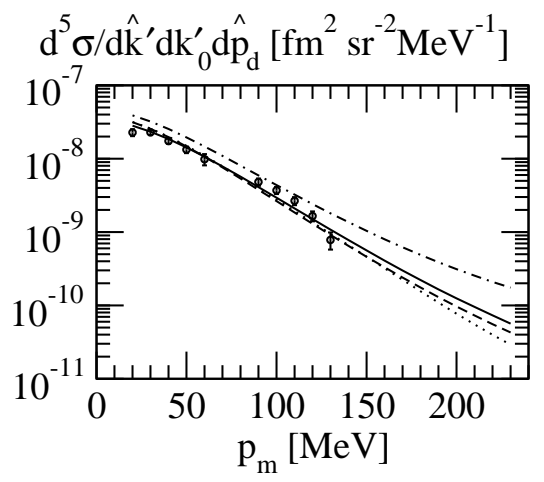

FIG. 50: The same as in Fig. 49 for $E_{e}=370 \mathrm{MeV}$ and $|\vec{Q}|=504 \mathrm{MeV} / \mathrm{c}$.

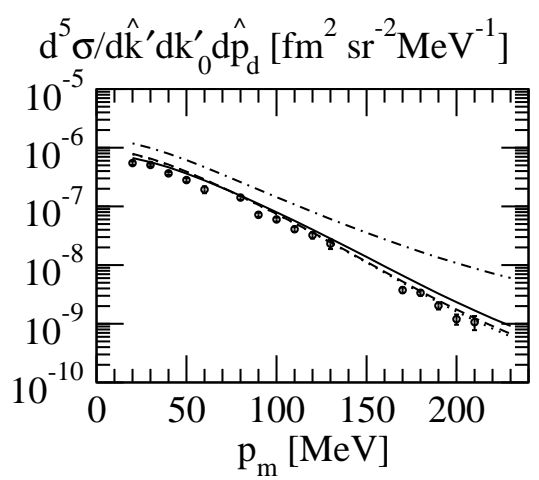

FIG. 51: The same as in Fig. 49 for $E_{e}=576 \mathrm{MeV}$ and $|\vec{Q}|=412 \mathrm{MeV} / \mathrm{c}$.

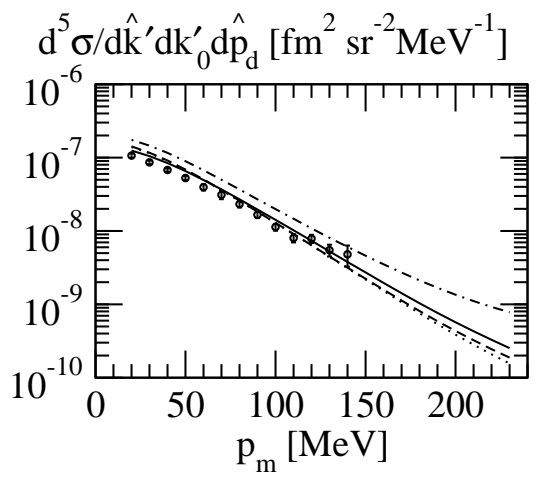

FIG. 52: The same as in Fig. 49 for $E_{e}=576 \mathrm{MeV}$ and $|\vec{Q}|=504 \mathrm{MeV} / \mathrm{c}$. 


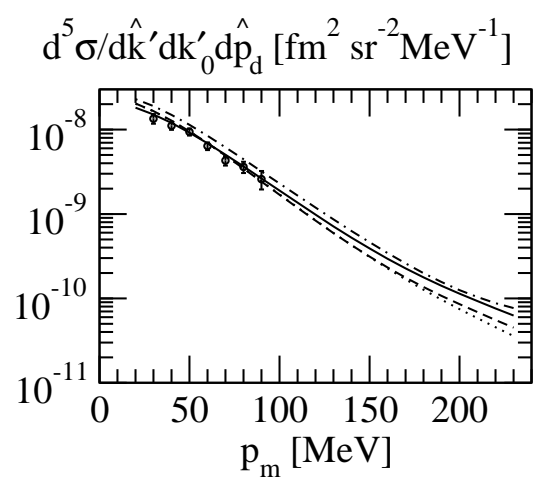

FIG. 53: The same as in Fig. 49 for $E_{e}=576 \mathrm{MeV}$ and $|\vec{Q}|=604 \mathrm{MeV} / \mathrm{c}$. 


\section{Nd radiative capture and the time reversed $\mathrm{Nd}$ photodisintegration of $3 \mathrm{~N}$ bound states.}

Photon angular distributions for pd capture have been measured for a wide range of energies. We show in Fig. 54 the cross section data in comparison to our theory with different dynamical ingredients. We rely either on the explicit MEC's for $\pi-$ and $\rho$-like exchanges or on the Siegert approach as described in section III B and show results either for the NN force AV18 alone or together with the UrbanaIX 3N force. We see an overall good agreement in the AV18 + UrbanaIX model together with explicit MEC's. Also the Siegert predictions for that choice of the interactions are similar. Since our MEC currents are not fully consistent to the forces, one cannot expect equality of these two approaches. At the higher energies the higher multipoles play a role. All the multipoles are kept in our Siegert approach only on the level of the single nucleon current. Nevertheless for the cross sections that Siegert approach does reasonably well in conjunction with the $3 \mathrm{~N}$ force. This is not the case for AV18 with Siegert, while AV18 together with MEC's is much closer to the data. We also would like to point out that the addition of the $3 \mathrm{~N}$ force decreases the cross section at the lower energies below $\approx 30 \mathrm{MeV}$ and increases it at the higher ones. In [157] we argued that this is not only a scaling effect with the $3 \mathrm{~N}$ binding energy as often claimed in the literature but at the higher energies it is also caused by the action of the $3 \mathrm{~N}$ force in the continuum.

In Fig. 55 we show photon angular distributions for nd capture around $10 \mathrm{MeV}$ neutron lab energy. The situation is very similar to the case of pd capture.

FIG. 54: The c.m. pd capture cross sections at various deuteron lab energies and four different dynamical inputs: MEC + AV18 (dashed line), Siegert + AV18 (dot-dashed line), MEC + AV18 + UrbanaIX (solid line), Siegert + AV18 + UrbanaIX (dotted line). Data at $10 \mathrm{MeV}$ are from [158], at 19.8 and 29.6 MeV from [159], at $95 \mathrm{MeV}$ from [160], at $200 \mathrm{MeV}$ circles from [161] and x-es from [169], and at $400 \mathrm{MeV}$ from [161].

Then there is a rich set of polarization observables in pd capture. Proton analyzing powers $A_{y}(p)$ at $E_{d}=10,200,300$ and $400 \mathrm{MeV}$ are shown in Fig. 56. At the deuteron lab energy 


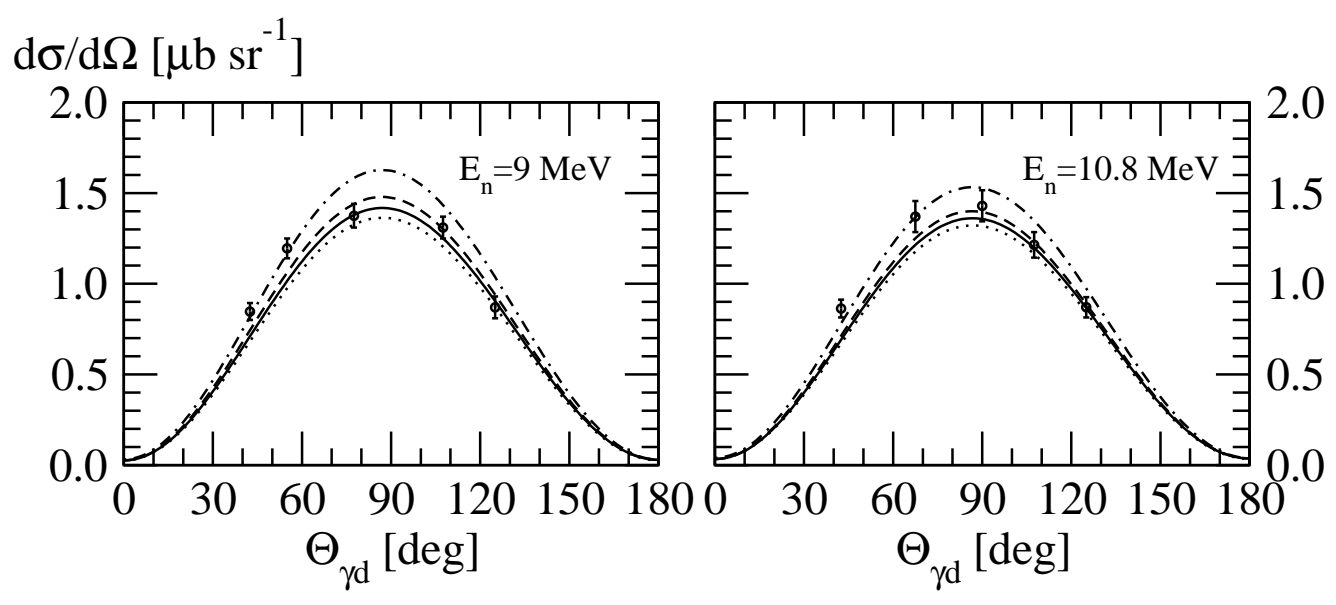

FIG. 55: The c.m. nd capture cross sections at 9.0 and $10.8 \mathrm{MeV}$ neutron lab energies. Curves as in Fig. 54. Data at 9.0 MeV and $10.8 \mathrm{MeV}$ are from [162].

of $10 \mathrm{MeV}$ the choice MEC+AV18+UrbanaIX comes closest to the data but nevertheless fails significantly at the smaller angles. For the three much higher energies the two curves with explicit MEC's come significantly closer to the data than the Siegert predictions but are too high at $E_{d}=200$ and $300 \mathrm{MeV}$ in relation to the data. Maybe it is accidental that there is a good agreement for the MEC predictions at $E_{d}=400 \mathrm{MeV}$.

Like for the proton analyzing power $A_{y}(p)$ we face a serious discrepancy for the deuteron vector analyzing power $\mathrm{iT}_{11}$ (in the spherical notation $\mathrm{iT}_{11}=\frac{\sqrt{3}}{2} \mathrm{~A}_{y}(\mathrm{~d})$ ). This is shown in Fig. 57. Again the MEC+AV18+UrbanaIX model comes closest to the data for the deuteron lab energies $\mathrm{E}_{d}=10,17.5,29,45$, and $200 \mathrm{MeV}$. At $\mathrm{E}_{d}=95 \mathrm{MeV}$ all our predictions show a strong slope not seen in the data. In view of the strong discrepancy and the relatively large experimental error bars a renewed, more precise measurement at this energy would be very useful to challenge improved theoretical approaches in the future.

Finally we look into the group of tensor analyzing powers. The spherical and cartesian notations are connected as

$$
\begin{aligned}
& A_{x x}=\sqrt{3} T_{22}-\frac{\sqrt{2}}{2} T_{20} \\
& A_{y y}=-\sqrt{3} T_{22}-\frac{\sqrt{2}}{2} T_{20} \\
& A_{x z}=-\sqrt{3} T_{21} .
\end{aligned}
$$

The observables $T_{20}, T_{21}$ and $T_{22}$ are displayed in Fig. 58. Overall there is a good agreement 


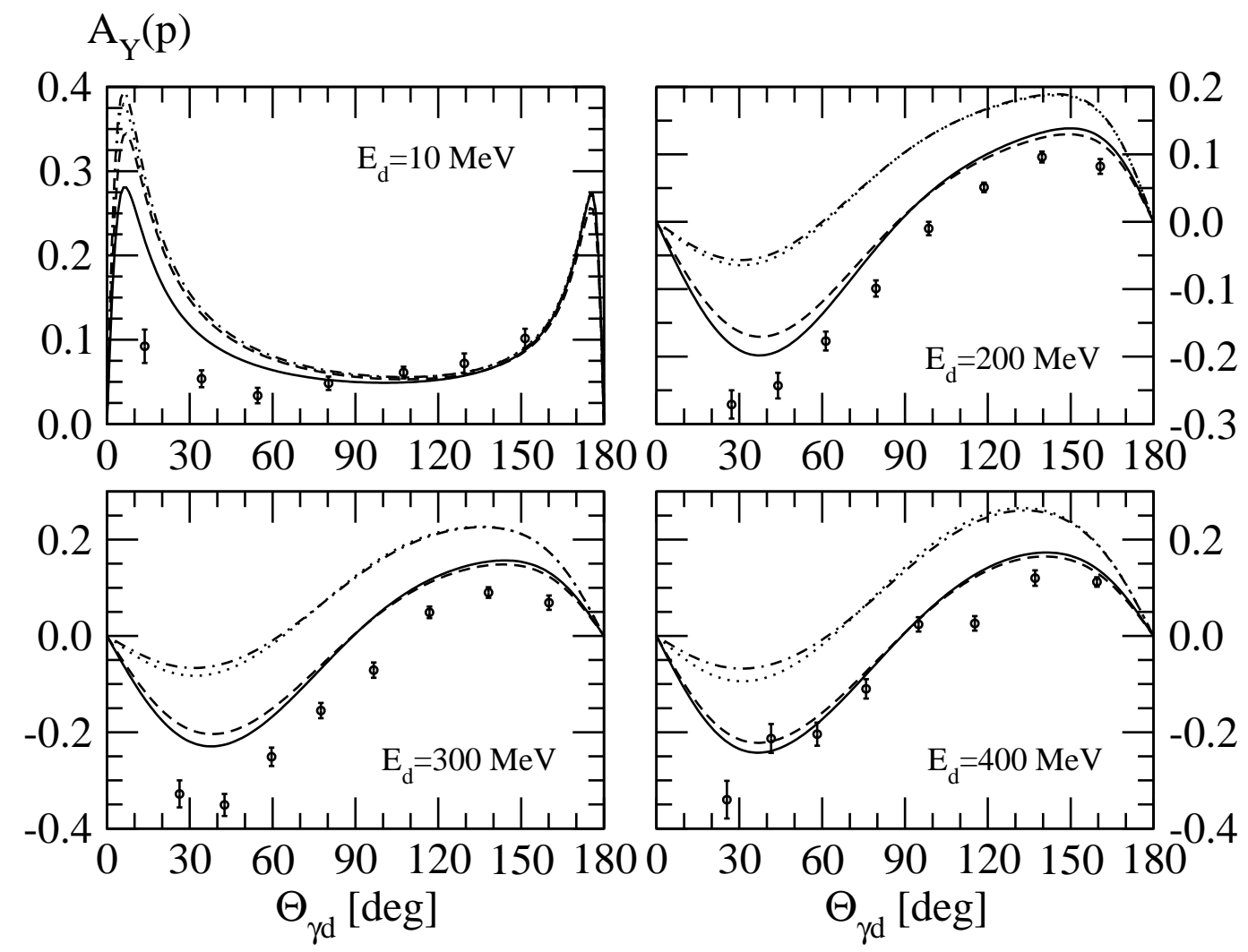

FIG. 56: The c.m. angular distributions for the proton analyzing powers $A_{y}(p)$ in pd capture at various deuteron lab energies. Curves as in Fig. 54. Data at $10 \mathrm{MeV}$ from [158], at 200, 300 and $400 \mathrm{MeV}$ from [161].

but the accuracy of the data does not allow a clear distinction among the four theoretical predictions. $\mathrm{T}_{21}$, and to an even larger extent $\mathrm{T}_{22}$, turn out to be quite independent to the dynamical input. In the case of $\mathrm{T}_{20}$ the explicit MEC picture reproduces the data at small angles better than the Siegert approach.

Next we show $\mathrm{A}_{y y}$ in Fig. 59. The data at the two lowest energies are fairly well described by the explicit MEC choice. In the case of $45 \mathrm{MeV}$ theory is somewhat too low and especially at very backward angles one misses the few data points totally. At $95 \mathrm{MeV}$ all our predictions are also too low. Finally, Fig. 60 shows $A_{x x}$ and $A_{z z}$ which agree fairly well with the explicit MEC approach.

In [66] the two-body photodisintegration of the $3 \mathrm{~N}$ bound state as well as the time reversed process have also been studied including a $\Delta$-isobar excitation. The selected results shown there are very similar to the ones displayed above. The difference in Fig. 58 to Fig. 11 of [66] 
FIG. 57: The c.m. angular distributions for the deuteron vector analyzing power i $\mathrm{T}_{11}$ in pd capture at various deuteron lab energies. Curves as in Fig. 54. Data at $10 \mathrm{MeV}$ from [158], at $17.5 \mathrm{MeV}$ from [167], at 29 and $45 \mathrm{MeV}$ from [168], at $95 \mathrm{MeV}$ from [160], and at $200 \mathrm{MeV}$ [169].

FIG. 58: The c.m. angular distributions for the tensor analyzing powers $\mathrm{T}_{20}, \mathrm{~T}_{21}$ and $\mathrm{T}_{22}$ for $\mathrm{pd}$ capture at low energies. Curves as in Fig. 54. Data at $10 \mathrm{MeV}$ from [158], and at $19.8 \mathrm{MeV}$ from [170](circles) and form [41] (squares).

is due to a wrong choice of angles in [66]. If replotted the outcome in [66] is quite similar to 
FIG. 59: The c.m. angular distributions for the tensor analyzing power $\mathrm{A}_{y y}$ for pd capture at various energies. Curves as in Fig. 54. Data at 17.5 MeV from [167], at $29 \mathrm{MeV}$ from [37] (square) and [168] (circles), at $45 \mathrm{MeV}$ from [168] (circles) and [171] (squares) and at $95 \mathrm{MeV}$ from [160].

the one shown above.

The pd and nd captures at very low energies ( 0-100 keV c.m. energies) have considerable astrophysical relevance for studies of stellar structure and evolution and of big-bang nucleosynthesis. Since single nucleon currents are insufficient to connect the dominant Sstate components of the two- and three-body bound states, small components of the wave functions acquire importance and even more the additional many-body currents. Therefore these reactions deserve a careful study. We refer to [26] for an introduction to these very low energy processes. In a series of papers [59, 92, 93, 163-166] these processes were investigated, experimentally and theoretically. In the most recent papers the two-body currents have been supplemented such, that they fulfill exactly the continuity equation related to the NN force AV18 and even three-nucleon currents have been added. We show in Fig. 61 the cross section and spin observables for pd radiative capture at $E_{c . m}$. $=3.33 \mathrm{MeV}$ obtained 
in $[92,93]$ with the AV18+UrbanaIX Hamiltonian model. These results document an important stride forwards, since the current used is fully consistent to the force model in the sense, that the continuity equation is exactly fulfilled. Some discrepancies in $A_{y}$ and $i T_{11}$ remain.
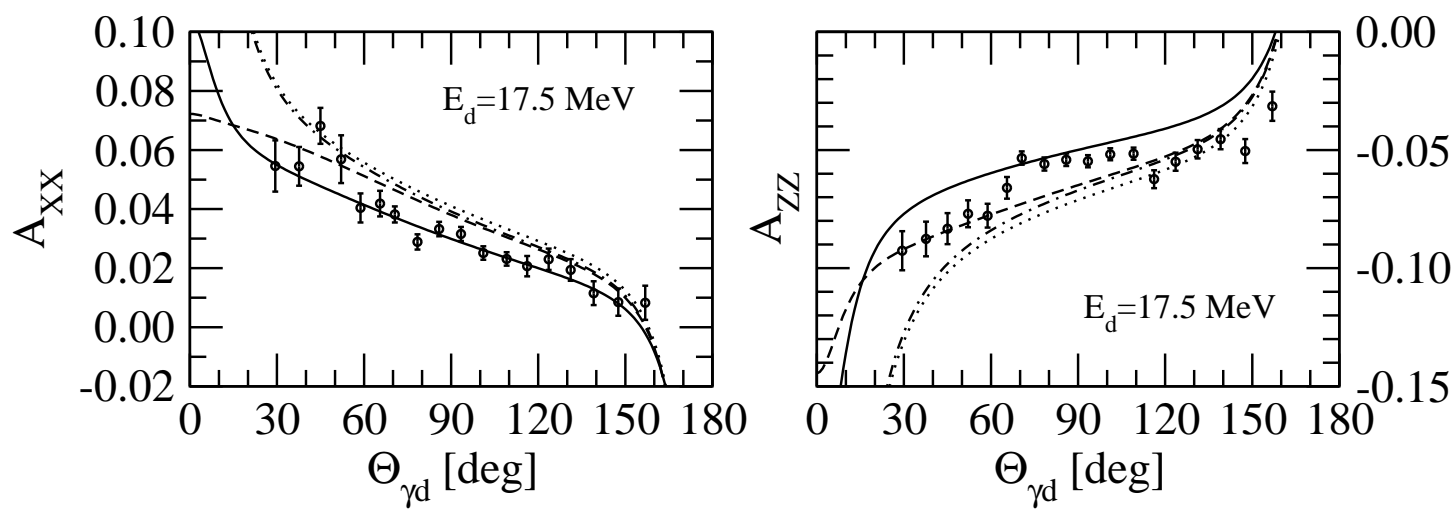

FIG. 60: The c.m. angular distributions for the tensor analyzing powers $\mathrm{A}_{x x}$ and $\mathrm{A}_{z z}$ for $\mathrm{pd}$ capture at 17.5 MeV. Curves as in Fig. 54. Data from [167].

Finally, we address photodisintegration of ${ }^{3} \mathrm{He}$. In Figs. 62 and 63 we display the cross section of the two-body $(\mathrm{pd})$ breakup in the c.m. system at two fixed angles, one at $\theta_{d}^{\text {c.m. }}=$ $90^{\circ}$ and one at $\theta_{d}^{l a b}=103.05^{\circ}$. The first one is shown for lower photon energies and the second one for higher ones, which beyond about $\mathrm{E}_{\gamma}=150 \mathrm{MeV}$ are strictly spoken outside the region where our theoretical framework is adequate. For the low energy region we display the predictions of Siegert and explicit MEC's for NN and NN + 3NF, respectively, while for the higher energy region only the explicit MEC predictions are shown. In both energy regions the MEC+AV18 + UrbanaIX predictions are in reasonably good agreement with the data except in the peak area around $\mathrm{E}_{\gamma}=10 \mathrm{MeV}$. This photon energy corresponds to $\mathrm{E}_{d}^{l a b}=13.47 \mathrm{MeV}$ in the time reversed pd capture reaction. As seen in Fig. 54, in that case there is a good agreement with the data at $\theta_{\gamma d}=90^{\circ}$. Thus we have to conclude that the data in Figs. 54 and the lower ones in Fig. 62 are inconsistent. This calls for an experimental clarification.

In the total pd breakup cross section given as a function of $E_{\gamma}$ in Fig. 64 there is a big spread in the experimental data, which makes any definite conclusion impossible. 

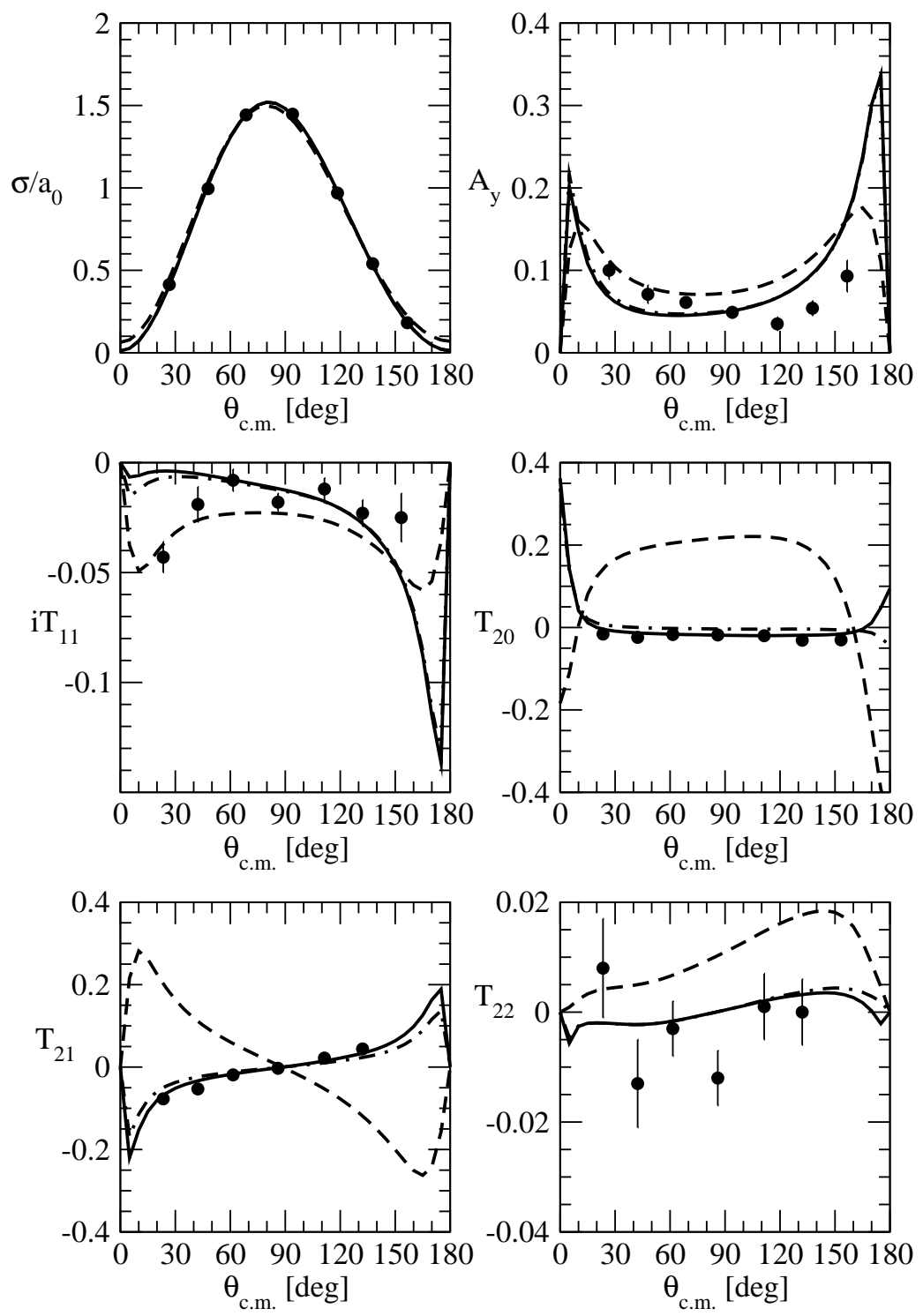

FIG. 61: The pd capture cross section and spin observables at $E_{c . m .}=3.33 \mathrm{MeV}$ obtained with the AV18+UrbanaIX Hamiltonian model $[92,93]$. The dashed, dot-dashed and solid curves correspond to the calculation with one-body only, with one- and two-body, and with one-, two- and three-body currents. For details see $[92,93]$. The data are from [158]. 


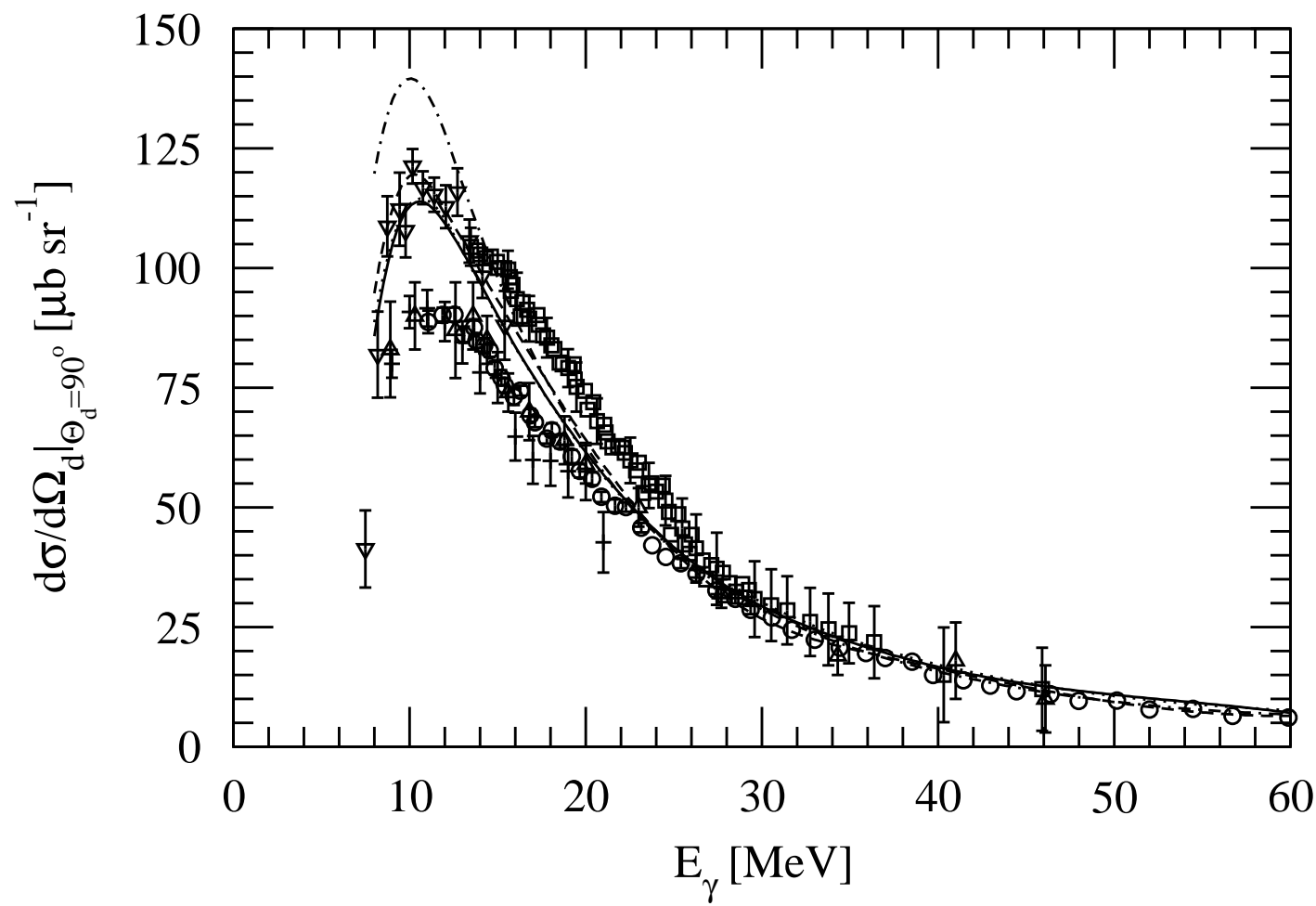

FIG. 62: Deuteron angular distribution for the process ${ }^{3} \mathrm{He}(\gamma, d) p$ at $\theta_{d}^{c . m .}=90^{\circ}$ as a function of the photon lab energy $E_{\gamma}$. Curves as in Fig. 54. The dotted curve practically overlaps with the solid one. Since the kinematical shift from the laboratory to the c.m. system is not significant, we combine the data for the $90^{\circ}$ laboratory angle (up triangles [172]) with the ones for the $90^{\circ}$ c.m. angle (circles [173]). The squares are the data from [181], pluses from [182], and down triangles from [180]. 


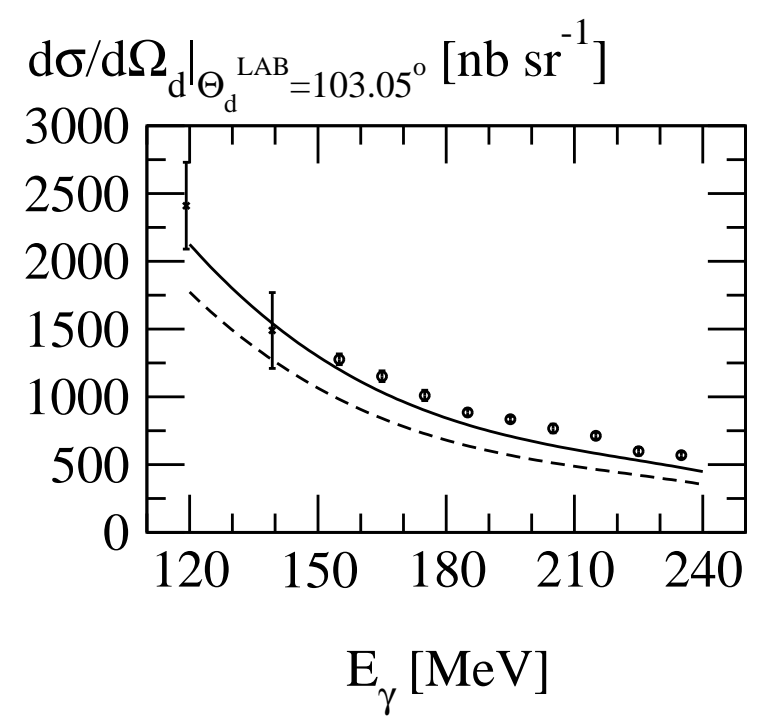

FIG. 63: Deuteron angular distribution for the process ${ }^{3} \mathrm{He}(\gamma, d) p$ at $\theta_{d}^{l a b}=103.05^{\circ}$ as a function of the photon energy $E_{\gamma}$. Curves show results of calculations with the AV18 + UrbanaIX (solid) and with the AV18 alone (dashed). Explicit $\pi$ - and $\rho$-like MEC's are included in the current operator. Data are from [174] (x-es) and [175] (circles). 


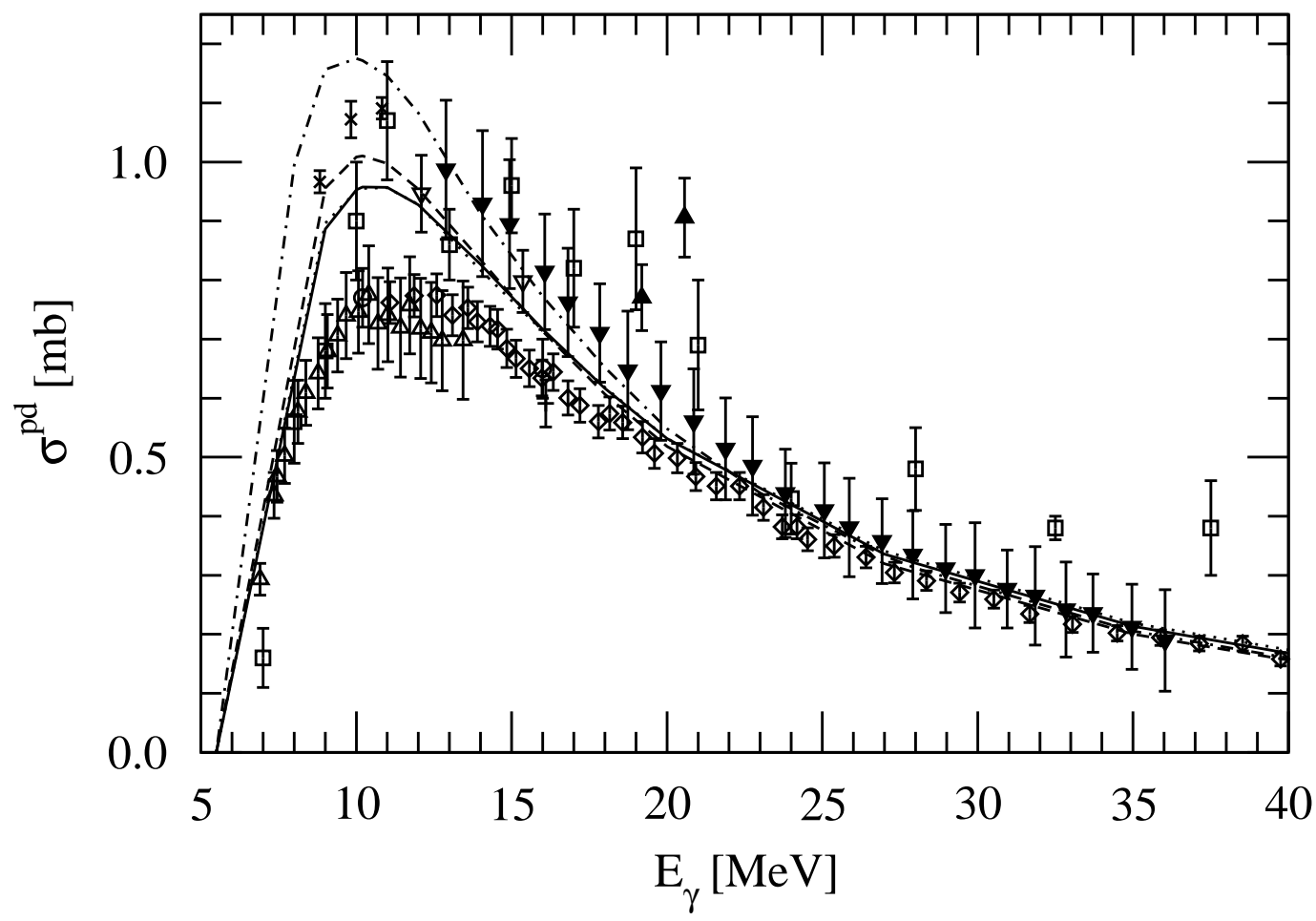

FIG. 64: Total ${ }^{3} \mathrm{He}(\gamma, \mathrm{p}) \mathrm{d}$ cross sections. Curves as in Fig. 54. The predictions with UrbanaIX both for Siegert and MEC (dotted and solid, respectively) are practically overlapping. Data are from: [176] (squares), [177] (up triangles), [159] (down triangles), [178] (full up triangles), [173] (diamonds), [179] (crosses), [180] (x-es), [181] (full down), [183] (circles). 


\section{E. Three-nucleon photodisintegration of the ${ }^{3} \mathrm{He}$}

We display in Fig. 65 the total 3N breakup cross section as a function of the photon energy in the lab system. There is again a big spread in the experimental data which precludes any definite conclusion. Especially the quick decline of one group of $3 \mathrm{~N}$ breakup data in comparison to our theoretical predictions is challenging, both for experiment and theory.

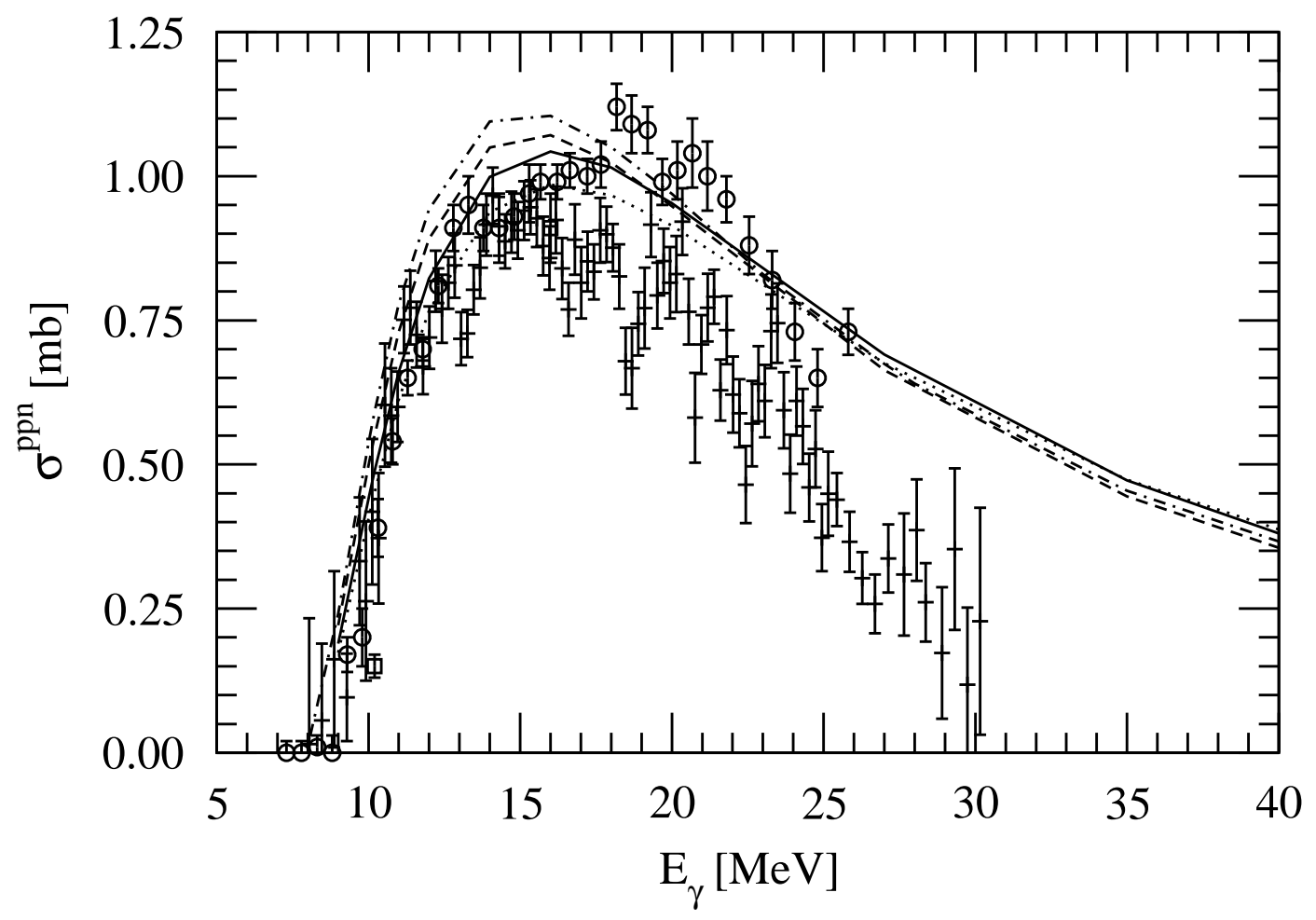

FIG. 65: Total ${ }^{3} \mathrm{He}(\gamma, \mathrm{pp}) \mathrm{n}$ cross sections. Curves as in Fig. 54. Data are from: [184] (crosses), [185] (circles), and [183] (squares).

In case of exclusive data we are only aware of two measurements. In Figs. 66 and 67 we show the four-fold differential $3 \mathrm{~N}$ breakup cross sections $\frac{d^{4} \sigma}{d \Omega_{1} d \Omega_{2}}$ for the detection of two nucleons in coincidence. In Fig. 66 the dependence on the incoming photon energy of the angular configurations, called LR-RL, LL-RR and LL-RL + LR - RR in [186], are investigated and compared to two of our predictions. Unfortunately we lack the information about angle acceptances of the detectors and therefore the comparison could be only a rough and qualitative one. 
In Fig. 67 the four-fold differential cross sections are displayed as a function of the opening angle between the outgoing neutron and a proton. Again the exact experimental conditions were not accessible to us and therefore the comparison of our point geometry theory and data has to be taken with care. These two experiments clearly demonstrate that data of those types are accessible. Renewed measurements with experimentalists and theoreticians working closely together would be very valuable to test the complex interplay of the dynamical ingredients. Recently, in [187] the three-nucleon photonuclear reactions with $\Delta$-isobar excitation have been analyzed with similar results to the ones shown above.

To close this section, we would like to draw attention to a benchmark calculation of the three-nucleon photodisintegration [188], where the LIT method has been compared to our momentum space Faddeev treatment. The agreement was quite good. We think that due to the very complex dynamics and the numerical challenges such benchmarks are necessary to make sure that the theoretical predictions really reflect exactly the dynamical input and justify the strong efforts of experimental groups. 

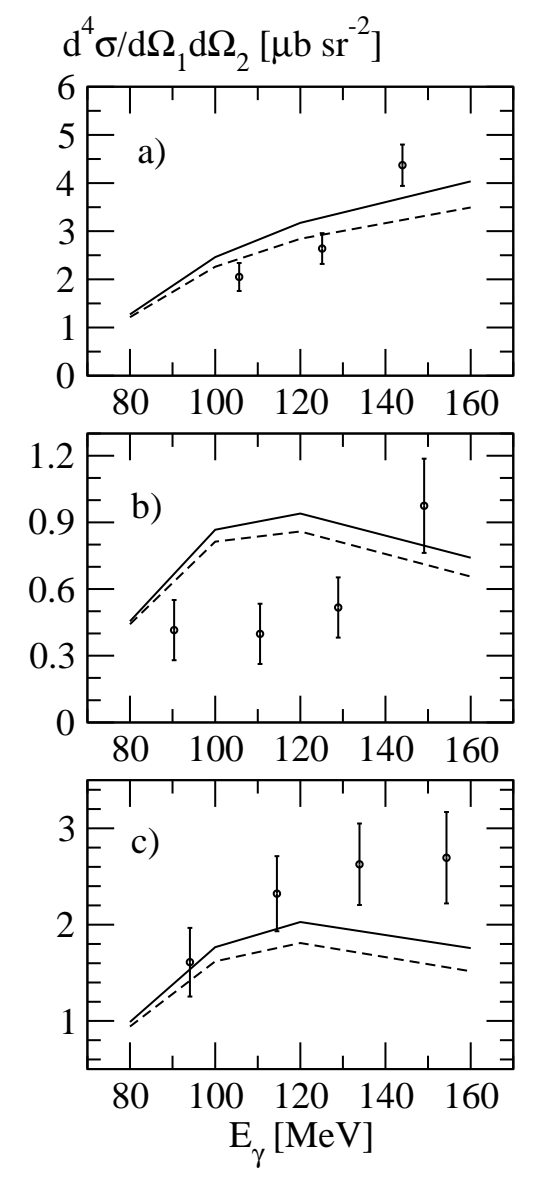

FIG. 66: The four-fold differential cross sections $\frac{d^{4} \sigma}{d \Omega_{1} d \Omega_{2}}$ for the ${ }^{3} \mathrm{He}(\gamma, \mathrm{pp}) \mathrm{n}$ process as a function of $E_{\gamma}$ in comparison to data from [186] for the angular configurations LR-RL $\left(\Theta_{1}=81.0^{\circ}, \Phi_{1}=\right.$ $\left.0.0^{\circ}, \Theta_{2}=80.3^{\circ}, \Phi_{2}=180.0^{\circ}\right)(\mathrm{a}), \operatorname{LL}-\mathrm{RR}\left(\Theta_{1}=92.2^{\circ}, \Phi_{1}=0.0^{\circ}, \Theta_{2}=91.4^{\circ}, \Phi_{2}=180.0^{\circ}\right)(\mathrm{b})$ and LL-RL+LR-RR $\left(\Theta_{1}=91.7^{\circ}, \Phi_{1}=0.0^{\circ}, \Theta_{2}=80.9^{\circ}, \Phi_{2}=180.0^{\circ}\right)$ and $\left(\Theta_{1}=81.5^{\circ}, \Phi_{1}=\right.$ $\left.0.0^{\circ}, \Theta_{2}=90.8^{\circ}, \Phi_{2}=180.0^{\circ}\right)$ (c). The solid curve is for AV18+UrbanaIX+MEC, the dashed curve for AV18+MEC. 

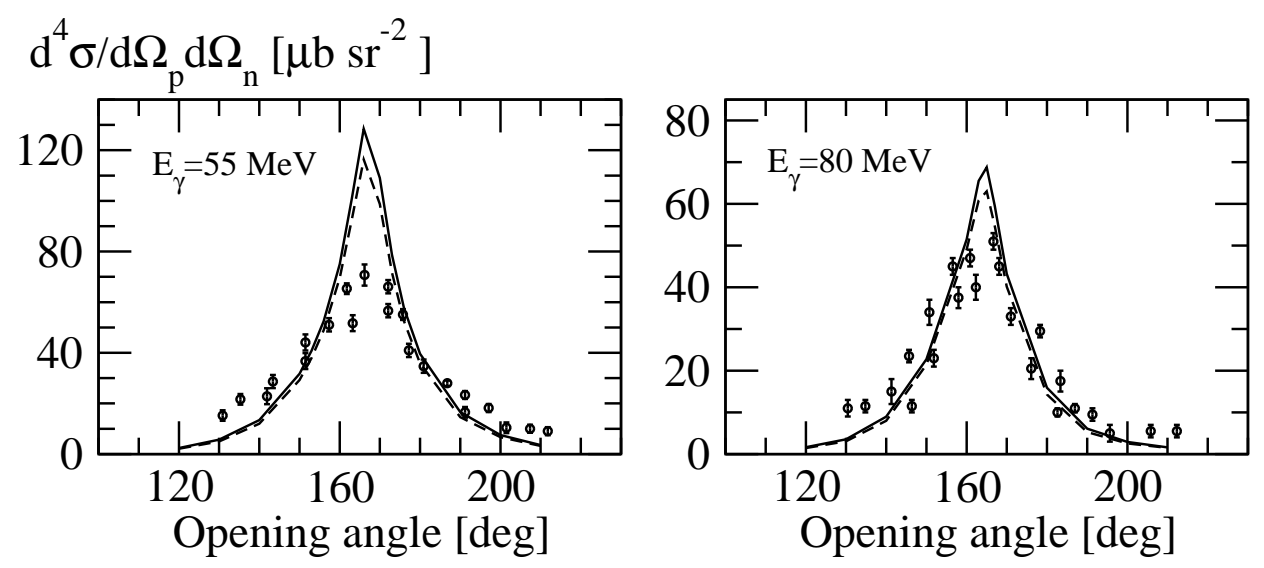

FIG. 67: The four-fold differential cross sections $\frac{d^{4} \sigma}{d \Omega_{1} d \Omega_{2}}$ against the opening angle at $E_{\gamma}=55$ (a) and $80 \mathrm{MeV}$ (b) for the ${ }^{3} \mathrm{He}(\gamma, \mathrm{pn}) \mathrm{p}$ process in comparison to data from [189]. The data in (b) were taken at $E_{\gamma}=85 \mathrm{MeV}$. Curves as in Fig.66. 


\section{PREDICTIONS}

The nuclear forces, on which we base our predictions in this review, AV18 and UrbanaIX, describe the whole wealth of NN data and reproduce the ${ }^{3} \mathrm{H}$ and the ${ }^{4} \mathrm{He}$ binding energies with high accuracy. This makes them a very often used tool for predictions in nuclear systems. It is certainly the "state-of-the-art" of the traditional approach to nuclear physics. We also employ the $\pi$ - and $\rho$-like two-body currents, which are linked to AV18 using the continuity equation and in this sense consistent. These currents are considered the dominant ones. These dynamical ingredients should already describe a wide range of processes. Obviously it is important to challenge this scenario and to find its limitations. In this section we go beyond the comparison to existing data and propose additional observables that will probe the dynamics more stringently. This is, of course, an incomplete and a subjective list but we hope that it can nevertheless guide future experimental efforts.

\section{A. Inclusive electron scattering on ${ }^{3} \mathrm{He}$}

In section VIB we showed data for the helicity asymmetries. They depend on the initial

${ }^{3}$ He spin direction and on the two response functions $\tilde{R}_{T^{\prime}}$ and $\tilde{R}_{T L^{\prime}}$. Their measurement by itself appears to be interesting, since they show a great sensitivity to the dynamical input as is illustrated in Figs. 68- 71. Especially interesting appears $\tilde{R}_{T L^{\prime}}$ for ${ }^{3} \mathrm{He}$, which in addition exhibits a strong variation in shape from $|\vec{Q}|=200$ over 300 and 400 to $500 \mathrm{MeV} / \mathrm{c}$. 

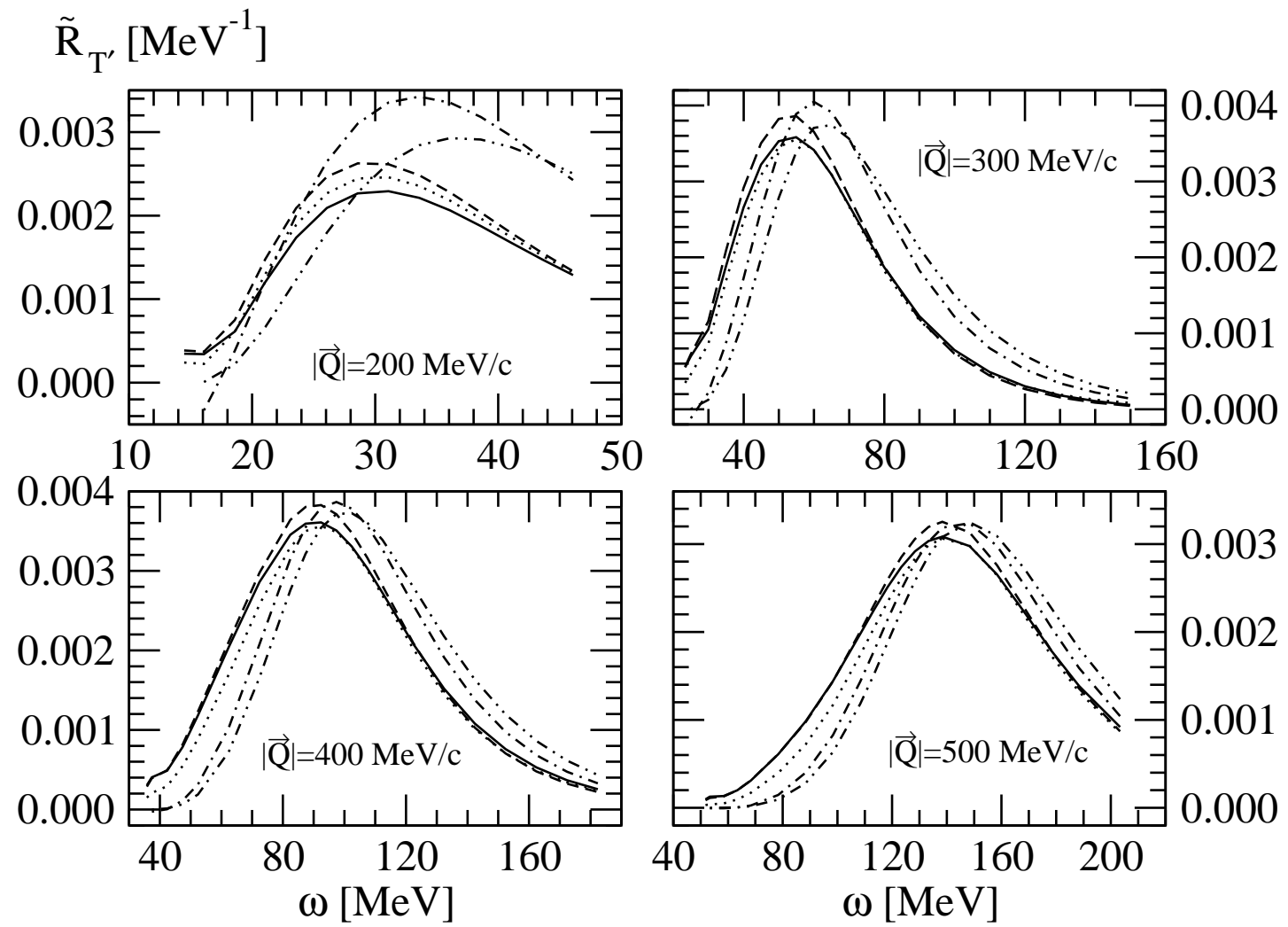

FIG. 68: The response function $\tilde{R}_{T^{\prime}}$ of ${ }^{3} \mathrm{H}$. Curves as in Fig. 23. 

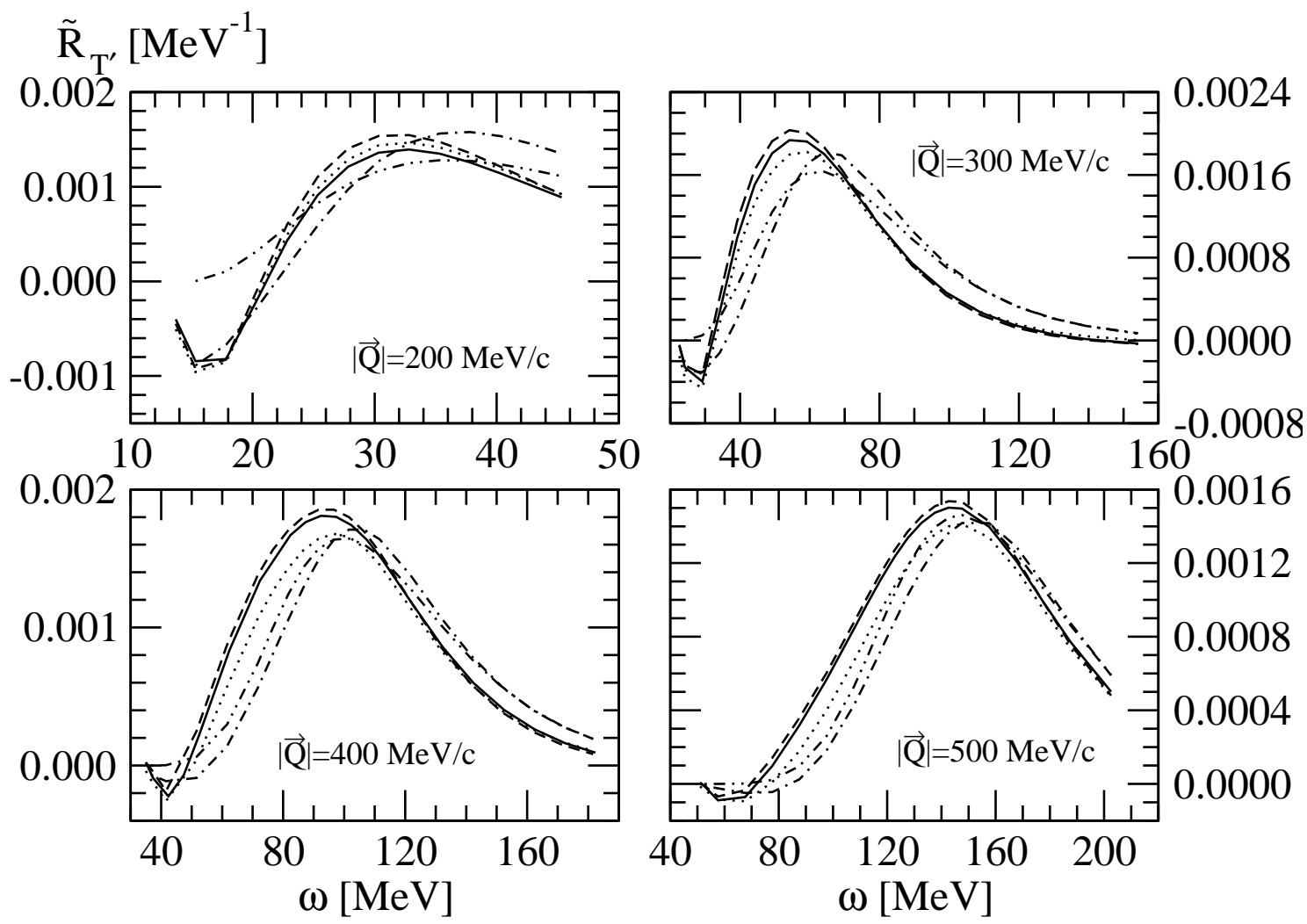

FIG. 69: The response function $\tilde{R}_{T^{\prime}}$ of ${ }^{3} \mathrm{He}$. Curves as in Fig. 23. 

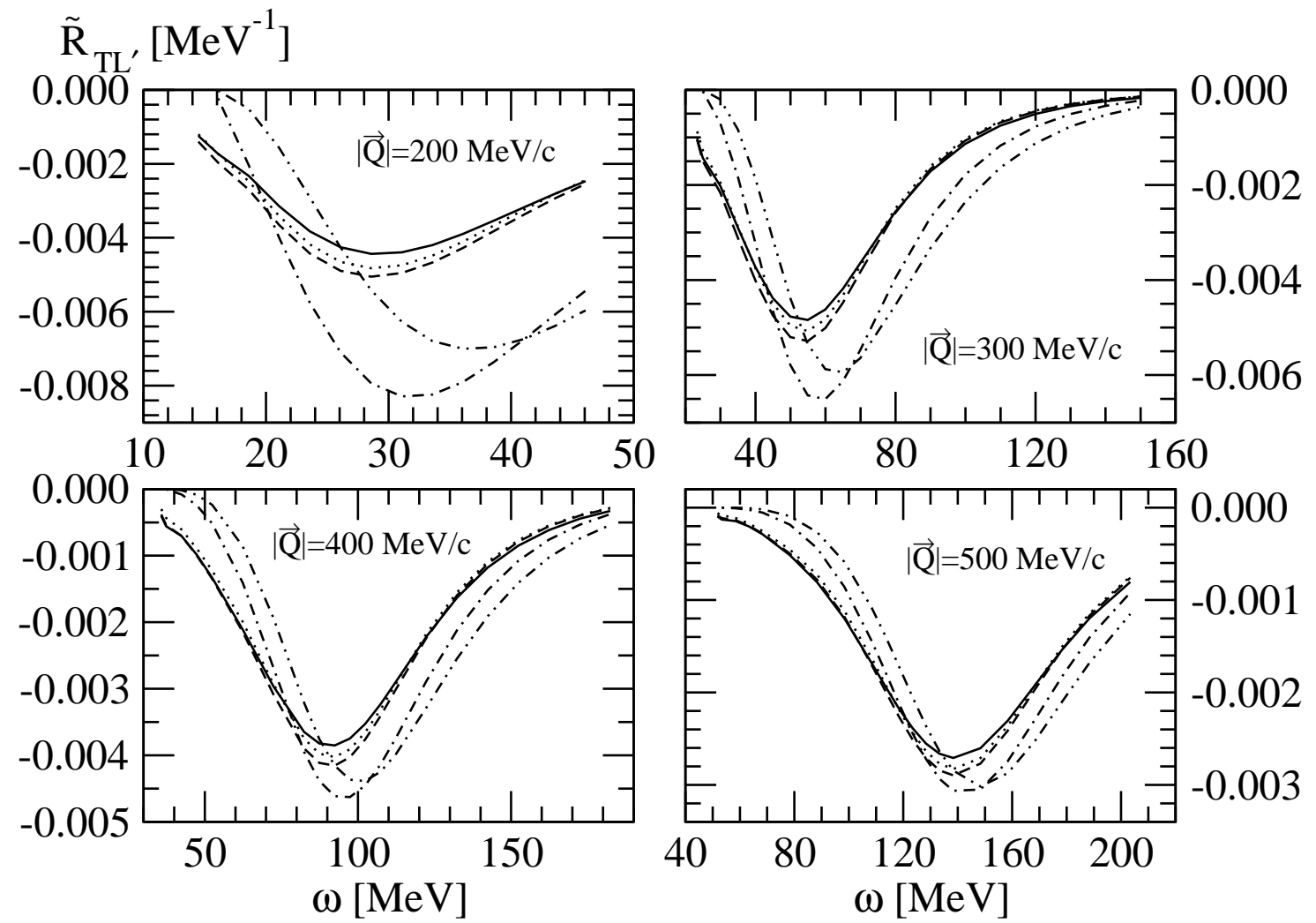

FIG. 70: The response function $\tilde{R}_{T L^{\prime}}$ of ${ }^{3} \mathrm{H}$. Curves as in Fig. 23. 

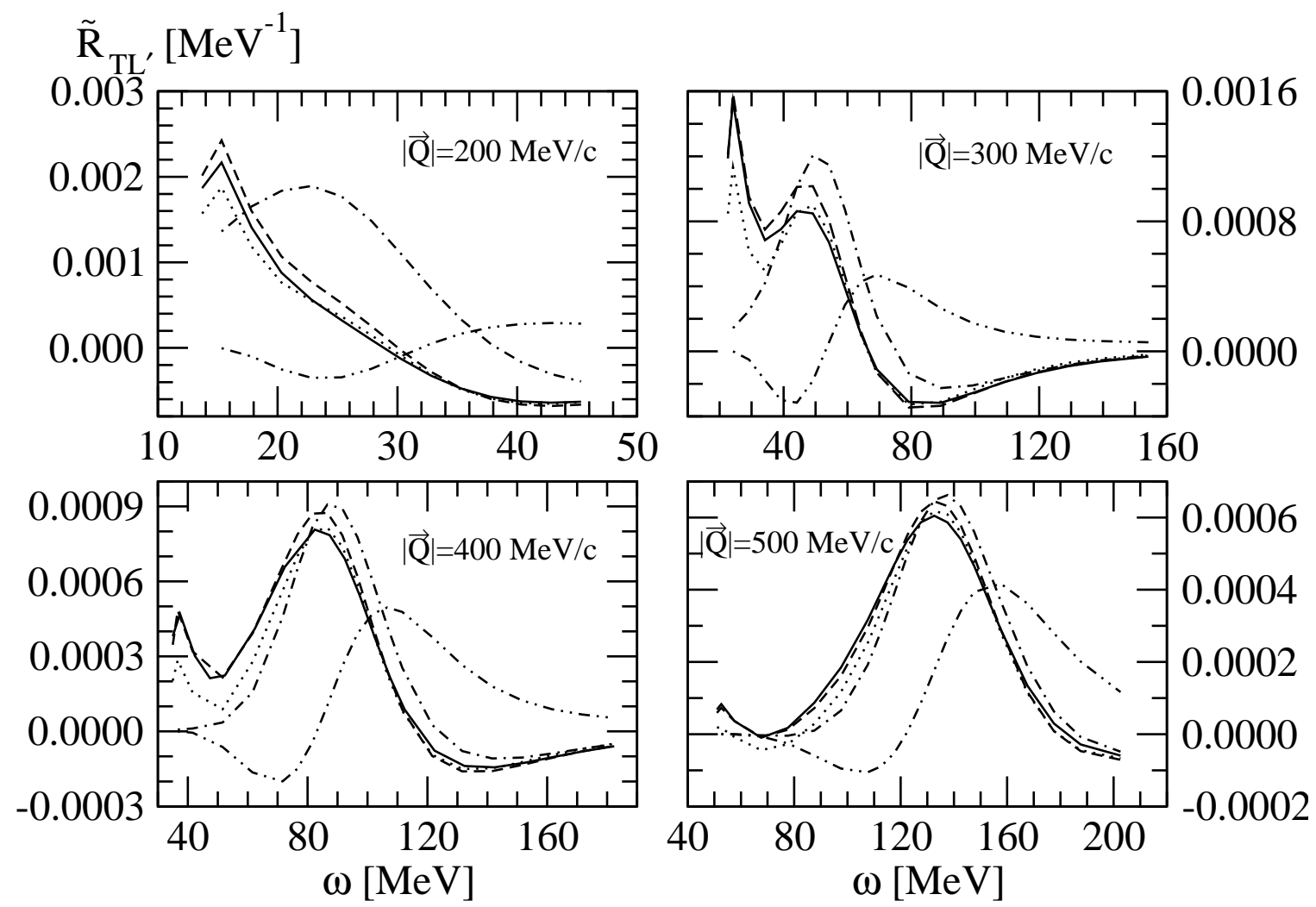

FIG. 71: The response function $\tilde{R}_{T L^{\prime}}$ of ${ }^{3} \mathrm{He}$. Curves as in Fig. 23. 


\section{B. Electron induced pd breakup of ${ }^{3} \mathrm{He}$}

In view of the discrepancies between theory and data displayed in section VIC it appears advisable to repeat measurements and to study that process more systematically. In the case of proton knockout there are cases where FSI is negligible and the MEC's we use do not contribute either. Therefore the angular distribution of the proton around quasi-elastic kinematics is determined by the simplest ingredients: the ${ }^{3} \mathrm{He}$ wave function, the single nucleon current and the deuteron wave function. It appears very natural to us that this most simple scenario should be tested in the first place. To the best of our knowledge this has not been done up to now. Further, in other cases FSI and/or 3NF effects show up. This is illustrated in Figs. 72-74. The cross sections shown in Fig. 72 displays three electron configurations (see figure caption). In the left one the FSI effect alone is insignificant and is then strongly modified by the inclusion of MEC and the 3NF. In the middle one PWIA is essentially sufficient and in the right one FSI is significant but the addition of the $3 \mathrm{NF}$ has no further effect. This quite different behavior is of course present in the two dominant response functions $R_{L}$ and $R_{T}$ displayed in Fig. 73. Finally for the sake of completeness the two very small responses $R_{T T}$ and $R_{T L}$ are shown in Fig. 74.

The situation is different in the deuteron peak area corresponding to proton angles around $240^{\circ}$. For our $|\vec{Q}|$-values below about $500 \mathrm{MeV} / \mathrm{c}$ our theory tells that it is not possible to knock out the deuteron without FSI. Though the effects of FSI and MEC's decrease going to higher $|\vec{Q}|$-values, sizable effects remain. This is illustrated in Figs. $75-77$. The cross section shown in Fig. 75 exhibits very strong shifts from the PWIAS predictions to the full results generated by FSI and 3NF. The detailed view into the underlying response functions $R_{L}$ and $R_{T}$ in Fig. 76 show that the MEC contributions in $R_{T}$ are different in the three configurations. Interesting is also the shift of the peak position in the third configuration for the full against the PWIAS result. Note also that in the cases shown in Fig. 75 the effect of the 3NF moves theory upwards while in Figs. 43-47 the 3NF effects cause a shift downwards. Apparently, there is an intricate dependence on the kinematical conditions. Again for the sake of completeness the two smallest response functions are displayed in Fig. 77. Precise new measurements would be very helpful to test existing and future dynamical inputs. Having the proton and the deuteron peak areas under control one would have covered essentially the full angular range. 

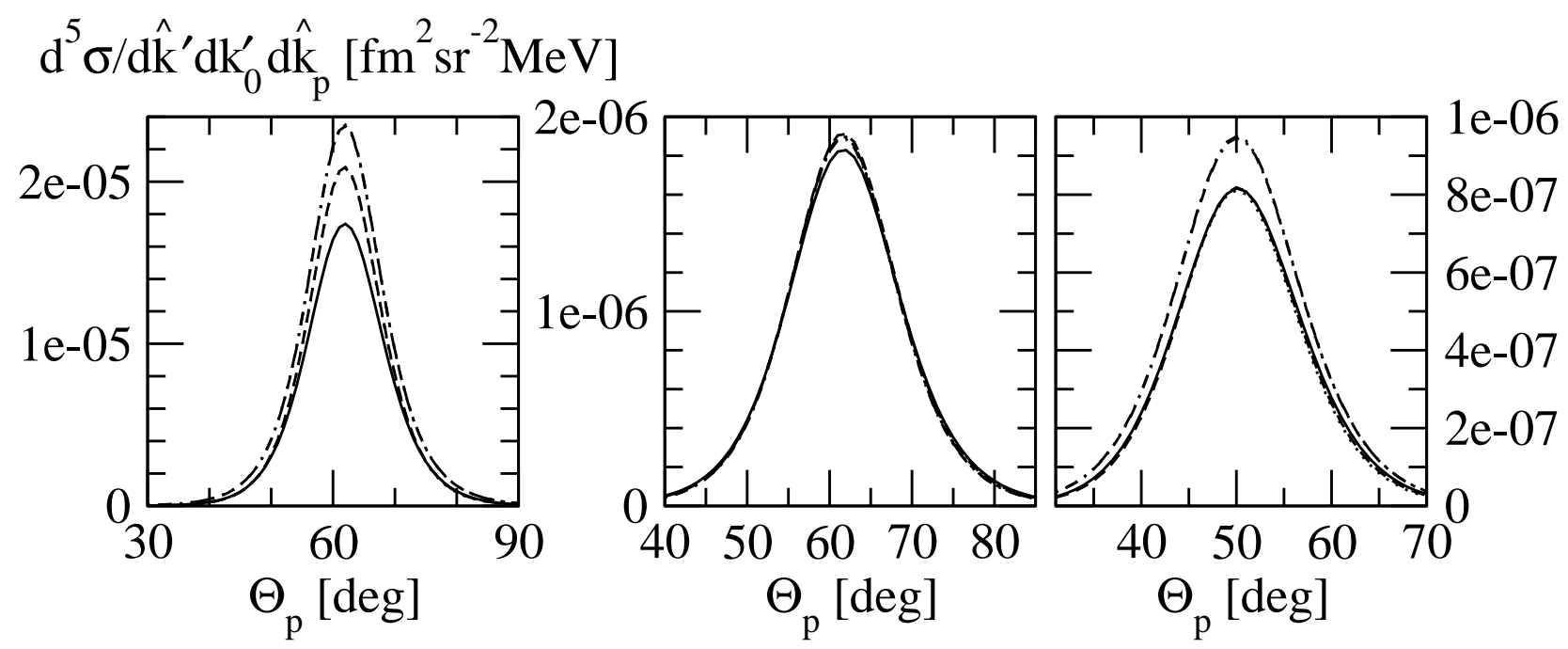

FIG. 72: Proton angular distribution in the vicinity of the proton knockout peak as a function of the lab proton angle $\theta_{p}$ (measured with respect to the electron beam) for three selected electron configurations: $k_{0}=854.5 \mathrm{MeV}, \vartheta=27.9^{\circ}, k_{0}^{\prime}=750.9 \mathrm{MeV}$ (left), $k_{0}=854.5 \mathrm{MeV}, \vartheta=35.5^{\circ}, k_{0}^{\prime}=$ 754.5 MeV (center) and $k_{0}=854.5 \mathrm{MeV}, \vartheta=35.7^{\circ}, k_{0}^{\prime}=652.3 \mathrm{MeV}$ (right). The different curves are PWIA (double-dot-dashed line), PWIAS (dot-dashed line - overlaps with PWIA), FSI (dotted line), FSI+MEC (dashed line) and FSI+MEC+3NF (solid line) predictions. 

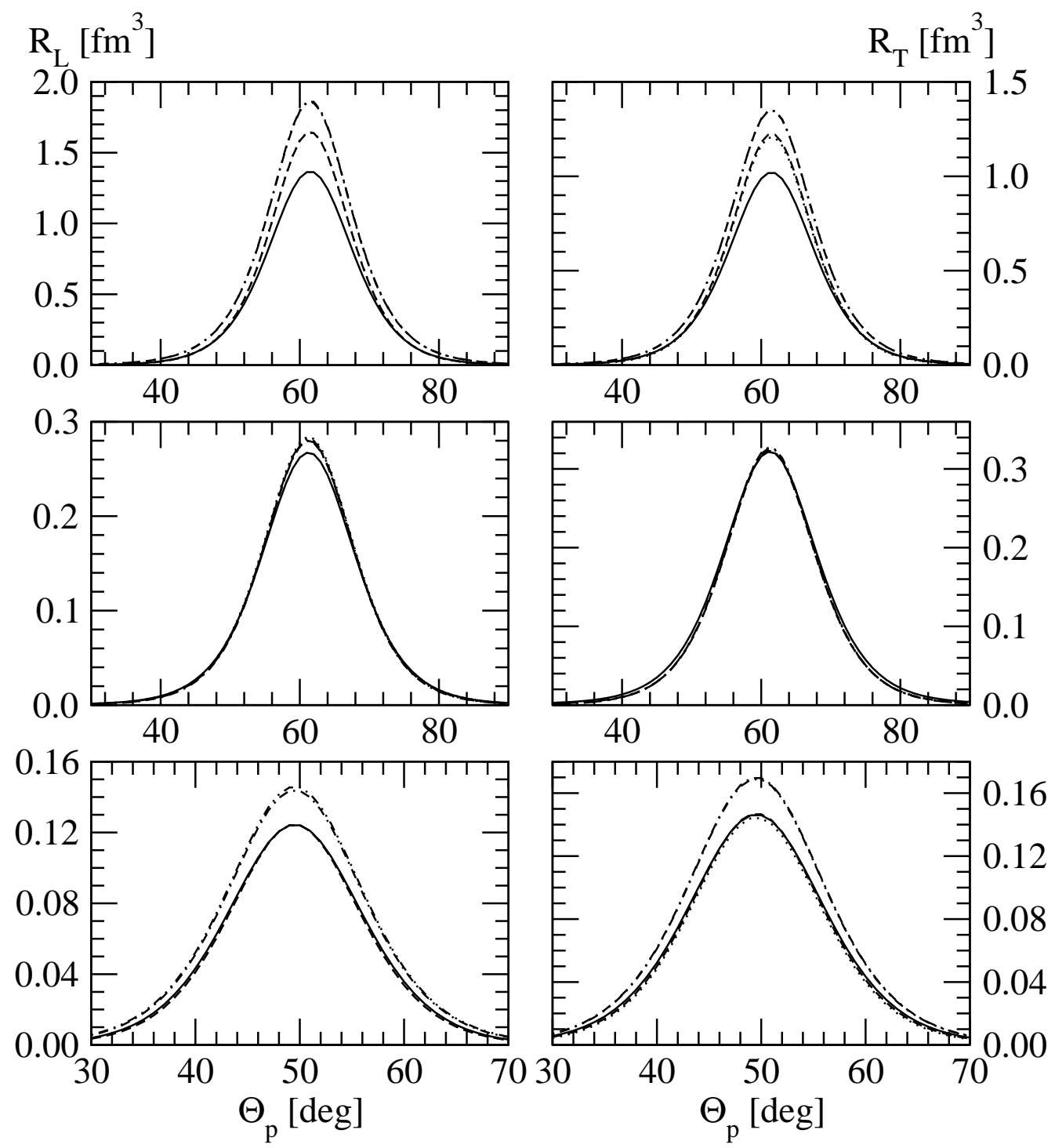

FIG. 73: The longitudinal $R_{L}$ (left) and transversal $R_{T}$ (right) responses in the vicinity of the proton knockout peak as a function of the lab proton angle $\theta_{p}$ for the same three electron configurations as in Fig. $72: k_{0}=854.5 \mathrm{MeV}, \vartheta=27.9^{\circ}, k_{0}^{\prime}=750.9 \mathrm{MeV}$ (upper row), $k_{0}=854.5 \mathrm{MeV}, \vartheta=$ $35.5^{\circ}, k_{0}^{\prime}=754.5 \mathrm{MeV}$ (middle row) and $k_{0}=854.5 \mathrm{MeV}, \vartheta=35.7^{\circ}, k_{0}^{\prime}=652.3 \mathrm{MeV}$ (bottom row). Curves as in in Fig. 72. 


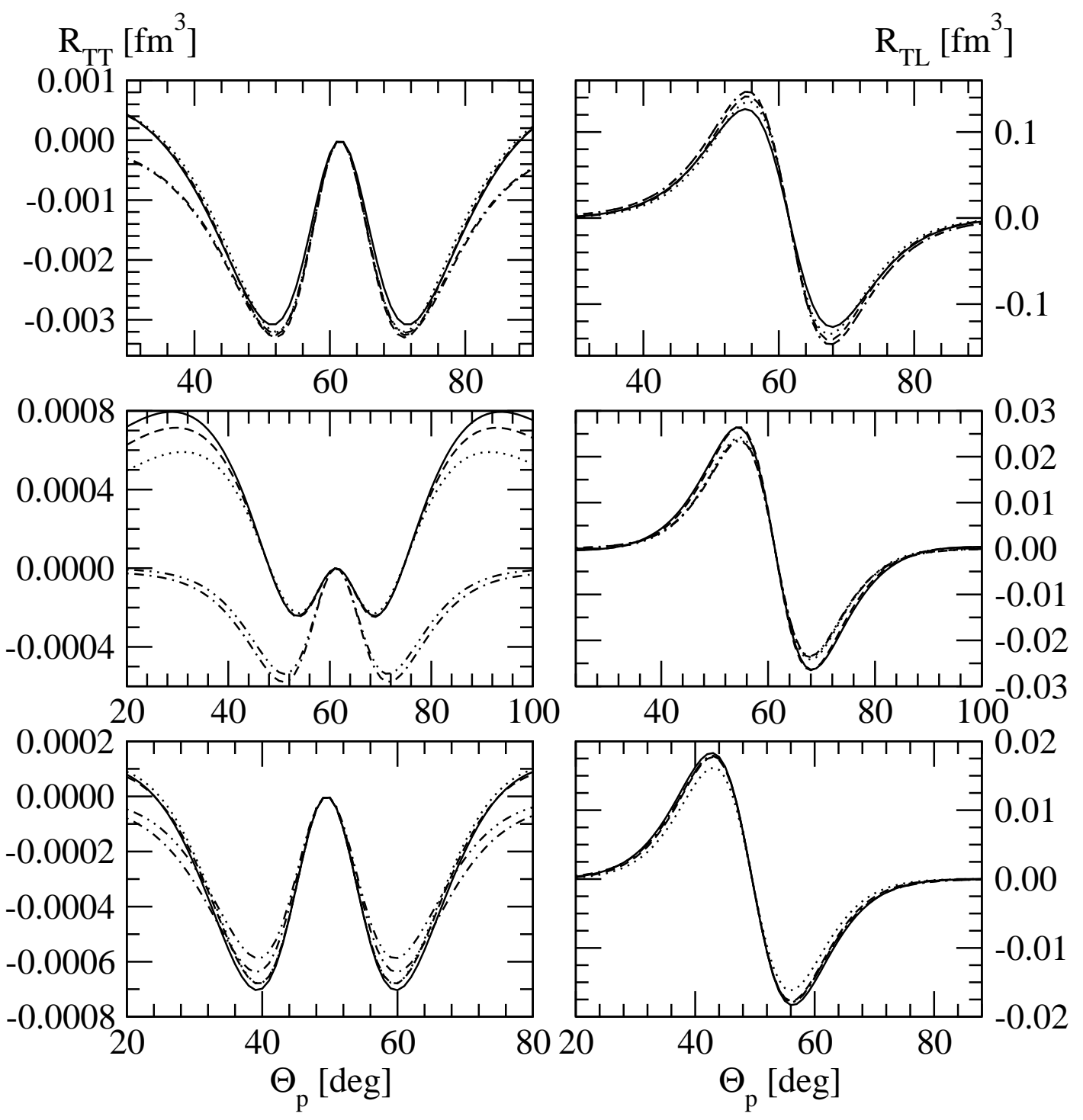

FIG. 74: The same as in Fig. 73 but for the $R_{T T}$ (left) and $R_{T L}$ (right) responses. 


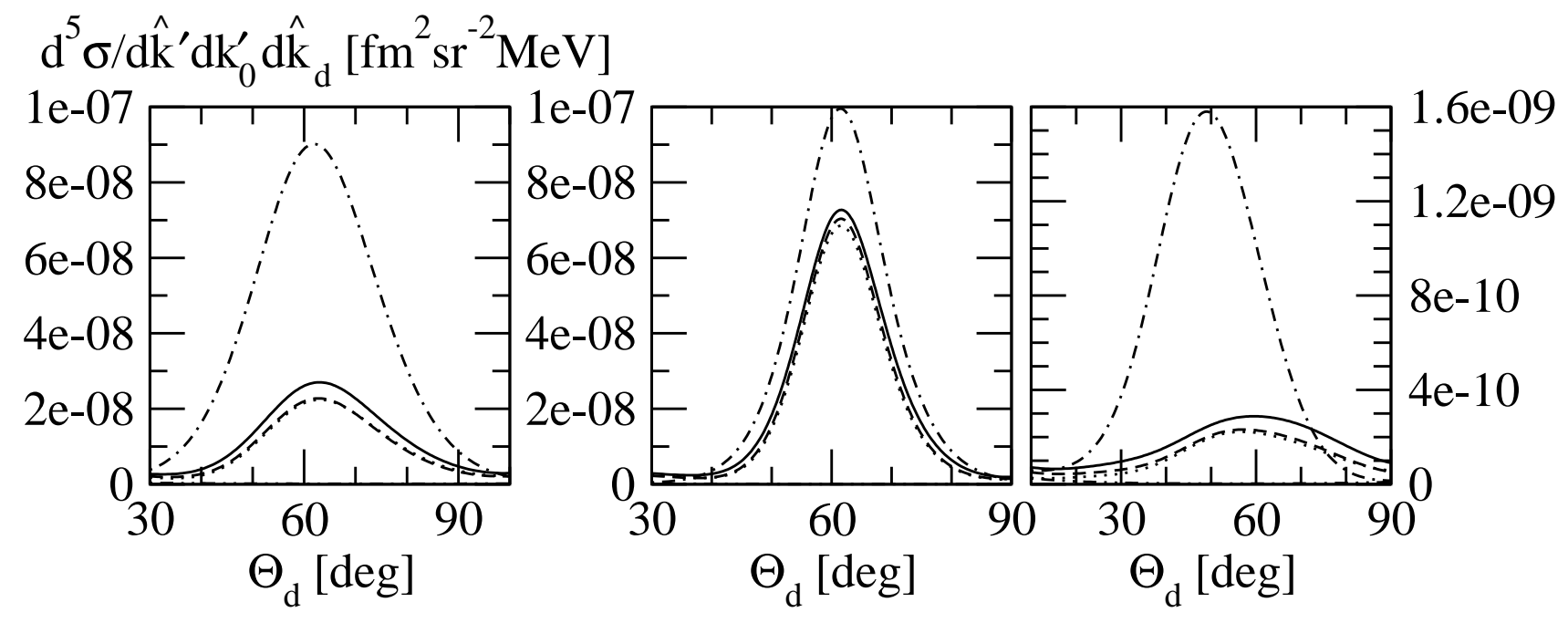

FIG. 75: Deuteron angular distribution in the vicinity of the deuteron knockout peak as a function of the lab deuteron angle $\theta_{d}$ for the same electron configurations as in Fig. $72: k_{0}=854.5 \mathrm{MeV}$, $\vartheta=27.9^{\circ}, k_{0}^{\prime}=750.9 \mathrm{MeV}$ (left), $k_{0}=854.5 \mathrm{MeV}, \vartheta=35.5^{\circ}, k_{0}^{\prime}=754.5 \mathrm{MeV}$ (center) and $k_{0}=854.5$ $\mathrm{MeV}, \vartheta=35.7^{\circ}, k_{0}^{\prime}=652.3 \mathrm{MeV}$ (right). Curves as in in Fig. 72 with the exception of PWIA, which is too small to be visible.

FIG. 76: The longitudinal $R_{L}$ (left) and transversal $R_{T}$ (right) responses in the vicinity of the deuteron knockout peak as a function of the lab deuteron angle $\theta_{d}$ for the same three electron configurations as in Fig. 75: $k_{0}=854.5 \mathrm{MeV}, \vartheta=27.9^{\circ}, k_{0}^{\prime}=750.9 \mathrm{MeV}$ (upper row), $k_{0}=854.5$ $\mathrm{MeV}, \vartheta=35.5^{\circ}, k_{0}^{\prime}=754.5 \mathrm{MeV}$ (middle row) and $k_{0}=854.5 \mathrm{MeV}, \vartheta=35.7^{\circ}, k_{0}^{\prime}=652.3 \mathrm{MeV}$ (bottom row). Curves as in in Fig. 72 .

FIG. 77: The same as in Fig. 76 but for the $R_{T T}$ (left) and $R_{T L}$ (right) responses. 


\section{Semiexclusive nucleon knockout processes}

The analysis of the process ${ }^{3} \mathrm{He}\left(e, e^{\prime} p\right) p n$ has often been done approximately using the concept of the spectral function. The underlying picture is simple. The photon is assumed to be absorbed by the knocked out proton and the remaining two nucleons are not involved in the photon absorption process nor do they interact with the knocked out proton. The only FSI kept is between the spectator neutron and proton. This is technically very easy to calculate, since beside the single nucleon current only the ${ }^{3} \mathrm{He}$ wave function and the NN $t$-matrix enter. Then only the two processes inside the dashed box of Fig. 78 are kept. This leads to the definition of the spectral function

$$
S(k, E)=\frac{m_{N} p}{2} \frac{1}{2} \sum_{m} \sum_{m_{1}, m_{2}, m_{3}} \int d \hat{p} \mid \sqrt{6}\left\langle\nu_{1} \nu_{2} \nu_{3}\right|\left\langle m_{1} m_{2} m_{3}\left|\left\langle\vec{p} \vec{k}\left|\left(1+t G_{0}\right)\right| \Psi_{i} m\right\rangle\right|^{2}(270)\right.
$$

The arguments of $S$ are the magnitude $k$ of the missing momentum

$$
k \equiv\left|\vec{Q}-\vec{k}_{p}\right|
$$

and the excitation energy $E$ (missing energy) of the undetected np pair. Nonrelativistically

$$
E \equiv \frac{p^{2}}{m_{N}}
$$

where $p$ is the relative momentum of the undetected nucleons. In addition we completed the notation by adding the isospin quantum numbers $\nu_{i}$. That strongly reduced treatment of FSI restricted only to the spectator nucleons 2 and 3 has already been introduced and denoted as FSI23 $\left(t \equiv t_{23}\right)$. One finds the relations [190]

$$
\begin{aligned}
& S(k, E)=\frac{1}{2} m_{N} p \frac{1}{\left(G_{E}\right)^{2}} \int d \hat{p} R_{L}(F S I 23) \\
& =\frac{1}{2} m_{N} p \frac{2 m_{N}^{2}}{|\vec{Q}|^{2}\left(G_{M}\right)^{2}} \int d \hat{p} R_{T}(F S I 23) .
\end{aligned}
$$

This form is convenient to compare to the treatment including the complete FSI and we define the quantities

$$
\begin{array}{r}
S_{L}(\text { Full })=\frac{1}{2} m_{N} p \frac{1}{\left(G_{E}\right)^{2}} \int d \hat{p} R_{L}(\text { Full }) \\
S_{T}(\text { Full })=\frac{1}{2} m_{N} p \frac{2 m_{N}^{2}}{|\vec{Q}|^{2}\left(G_{M}\right)^{2}} \int d \hat{p} R_{T}(\text { Full }),
\end{array}
$$

which enter directly into the semiexclusive cross section. 


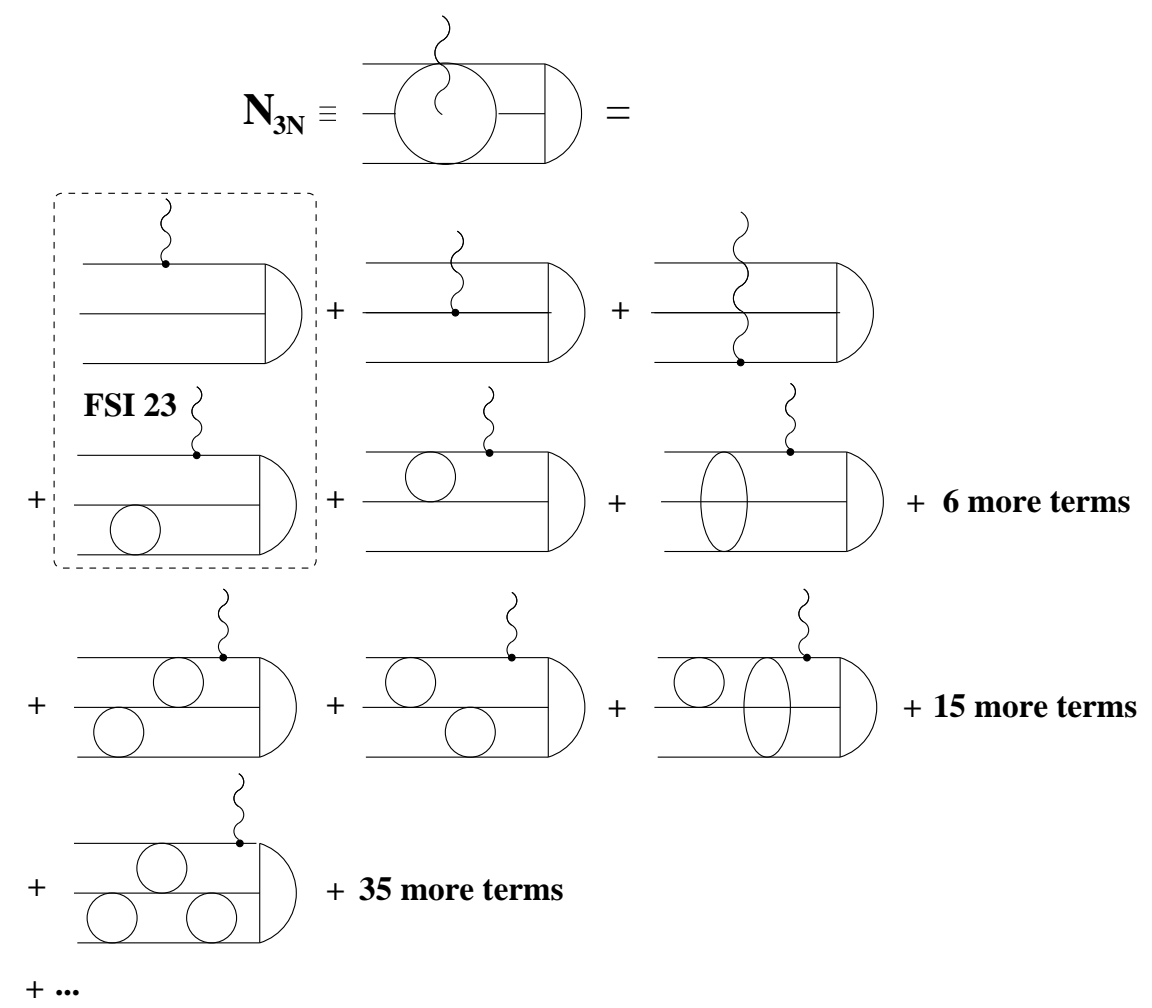

FIG. 78: Diagrammatic representation of the nuclear matrix element for the three-body electrodisintegration of ${ }^{3} \mathrm{He}$. The open circles and ovals represent the two-body $t$-matrices. Three horizontal lines between photon absorption and forces, and between forces describe free propagation. The half-moon symbol on the very right stands for ${ }^{3} \mathrm{He}$.

Using that simple picture of FSI23 the cross section factorizes into a kinematical factor, the electron proton cross section, and into the spectral function as shown below in (275).

We performed very recently a thorough investigation [190] on the validity of that approximation in the domain of nonrelativistic kinematics. We assumed the most favorable condition of parallel kinematics $\left(\vec{k}_{p} \| \vec{Q}\right)$. The result was that only for very small missing momenta $k \equiv p_{m}$ and missing energies $E$ the use of the spectral function is quantitatively justified. To each $(k, E)$ pair under parallel condition, $\omega \equiv Q_{0}$ and $|\vec{Q}|$ are connected by a quadratic equation. Then we found that there is a domain of $(k, E)$-values where, at least with increasing $|\vec{Q}|, S_{L}(F u l l)$ and $S_{T}(F u l l)$ approach $S$. But unexpectedly for our present insight even for quite small $k$-values but increasing $E$ values that simplified picture is invalid. We refer the interested reader for details to [190]. In any case again there are clear cut cases, where the concept of the spectral function is valid and they should be tested 
against precise data. Like in the quasi elastic proton peak for pd breakup, also in this case the theoretical ingredients are quite simple: just the ${ }^{3} \mathrm{He}$ wave function, the NN $t$-matrix, and the single nucleon current. The quantitative validity of this simple picture should be tested in the first place. But then it is also very interesting to probe the FSI, MEC and the $3 \mathrm{~N}$ force effects if one takes other $(k, E)$ pairs. We illustrate two of various cases from [190] in Fig. 79. The quantities (274) together with the spectral function are plotted as a function of the ejected proton energy $E_{1}$ for parallel kinematics $\vec{k}_{1} \| \vec{Q}$. We see in the left part of the Fig. 79 that at the upper end of $E_{1}$ the three curves approach each other, thus the spectral function concept works very well. The corresponding decreasing values of $\mathrm{k}$ and E for increasing $E_{1}$ are also indicated. A counter example is shown in the right part of the Fig. 79, where the use of the spectral function would be a very poor approximation.

In the case of the process ${ }^{3} \mathrm{He}\left(e, e^{\prime} n\right) p p$ the concept of the spectral function is useful only for $R_{T}$ but not for $R_{L}$. In the case of $R_{L}$ it is a totally insufficient approximation. The reason is of course the smallness of $G_{E}^{n}$ and the strong interference of the photoabsorption on the protons.

If the approximation leading to the spectral function was valid, it could be quite reliably used to extract electromagnetic nucleon form factors. The cross section factorizes as [190]

$$
\frac{d^{6} \sigma}{d k_{0}^{\prime} d \hat{k}^{\prime} d \hat{k}_{1} d E_{1}}=\sigma_{\text {Mott }}\left[v_{L}\left(G_{E}\right)^{2}+v_{T} \frac{|\vec{Q}|^{2}\left(G_{M}\right)^{2}}{2 m_{N}^{2}}\right] S(k, E) m_{N} k_{1} .
$$

This should be experimentally tested for $G_{E}^{p}$ and $G_{M}^{p}$ since the proton form factors are known and then be applied to $G_{M}^{n}$.

Since our investigation in [190] was restricted to the nonrelativistic domain, it does not provide information for the relativistic region. In $[148,191]$ the spectral function concept has been studied at higher $\omega$ and $|\vec{Q}|$-values. The verification of such an assumption requires a full-fledged relativistic framework including on top of FSI 3N forces and MEC's.

Now let us regard the semiexclusive process ${ }^{3} \mathrm{He}\left(\vec{e}, e^{\prime} n\right) p p$ for an initially polarized ${ }^{3} \mathrm{He}$ and polarized electron. The asymmetries (199) for parallel, $A_{\|}$, and perpendicular, $A_{\perp}$, orientation of the ${ }^{3} \mathrm{He}$ spin in relation to the photon direction are proportional to $\left(G_{M}^{n}\right)^{2}$ and $G_{E}^{n} G_{M}^{n}$, respectively, under the simplifying assumptions of PWIA and the restriction of the ${ }^{3}$ He state to the principal S state $[140,141,192]$. If that sensitivity survives for the full dynamics one can extract the neutron form factors. In Figs. 80 and 81 we provide $A_{\|}$and $A_{\perp}$ as a function of the ejected neutron energy for two kinematical conditions using different 
dynamical assumptions. We choose the most favorite configuration, where the neutron is ejected parallel to the photon. In both figures five curves are shown, PWIA, FSI23, FSI, FSI+MEC and FSI+MEC+3NF. For $\omega=50 \mathrm{MeV},|\vec{Q}|=300 \mathrm{MeV} / \mathrm{c}, Q^{2}=0.087(\mathrm{GeV} / \mathrm{c})^{2}$ shown in Fig. 80, $A_{\|}$for PWIA and FSI23 stays far off the results gained under FSI and with the further ingredients MEC and 3NF. Thus the extraction of $\left(G_{M}^{n}\right)^{2}$ under the simplifying assumptions of PWIA or FSI23 would require big corrections. In the case of $A_{\perp}$ that is also the case but $A_{\perp}$ is anyhow very small. Only out of curiosity we add the corresponding results for the proton ejection. Since the polarization of the proton inside polarized ${ }^{3} \mathrm{He}$ is very small, PWIA is of course far away from the other results. Then, in Fig. 81, for $\omega=150 \mathrm{MeV},|\vec{Q}|=500 \mathrm{MeV} / \mathrm{c}, Q^{2}=0.228(\mathrm{GeV} / \mathrm{c})^{2}$ the situation is quite different. All curves for $A_{\|}$coincide at the upper end of the neutron energy. This should allow one to extract $\left(G_{M}^{n}\right)^{2}$ without big corrections. However, for $A_{\perp}$ large corrections remain. In the case of the proton ejection the energy dependence of both asymmetries is totally different from the neutron ones what would be interesting to check experimentally. Also proton asymmetries reveal sizable $3 \mathrm{NF}$ effects. For the sake of completeness and orientation about the magnitudes of the cross sections we also include their values in the Figs. 80-81. These two examples just illustrate that both processes, neutron as well as proton emission, provide interesting tests of the dynamical inputs if accurate data can be gained.

In [141] the process ${ }^{3} \mathrm{He}\left(\vec{e}, e^{\prime} n\right) p p$ has been applied to extract $G_{E}^{n}$ for $Q^{2} \approx 0.35(\mathrm{GeV} / \mathrm{c})^{2}$. As expected, it turned out that the full FSI was required. The resulting $G_{E}^{n}$-value was quite different from the one extracted under the assumption that polarized ${ }^{3} \mathrm{He}$ is just a polarized neutron target [193]. Despite the inclusion of FSI the theoretical analysis in [141] was a bit overstretched since we relied on a nonrelativistic framework. The extracted value for $G_{E}^{n}$ might have differed a bit if relativity and MEC's had been included.

At higher $Q^{2}$-values the situation appears to be more favorable for the application of the theoretically simple approach offered by FSI23 as argued in [194, 195].

The cross section for the semi-exclusive $\vec{e}\left(\overrightarrow{{ }^{3} \mathrm{He}}, e^{\prime} N\right)$ reaction can also be cast in the following general form [196]

$$
\sigma(h, \vec{A})=\sigma_{0}\left[1+\vec{S} \cdot \vec{A}^{0}+h\left(A_{e}+\vec{S} \cdot \vec{A}^{\prime}\right)\right]
$$

where $\sigma_{0}$ is the unpolarized cross section, $A_{e}$ is the electron analyzing power, $\vec{A}^{0}$ the ${ }^{3} \mathrm{He}$ target analyzing power and $\vec{A}^{\prime}$ are the spin correlation parameters. The target analyzing 

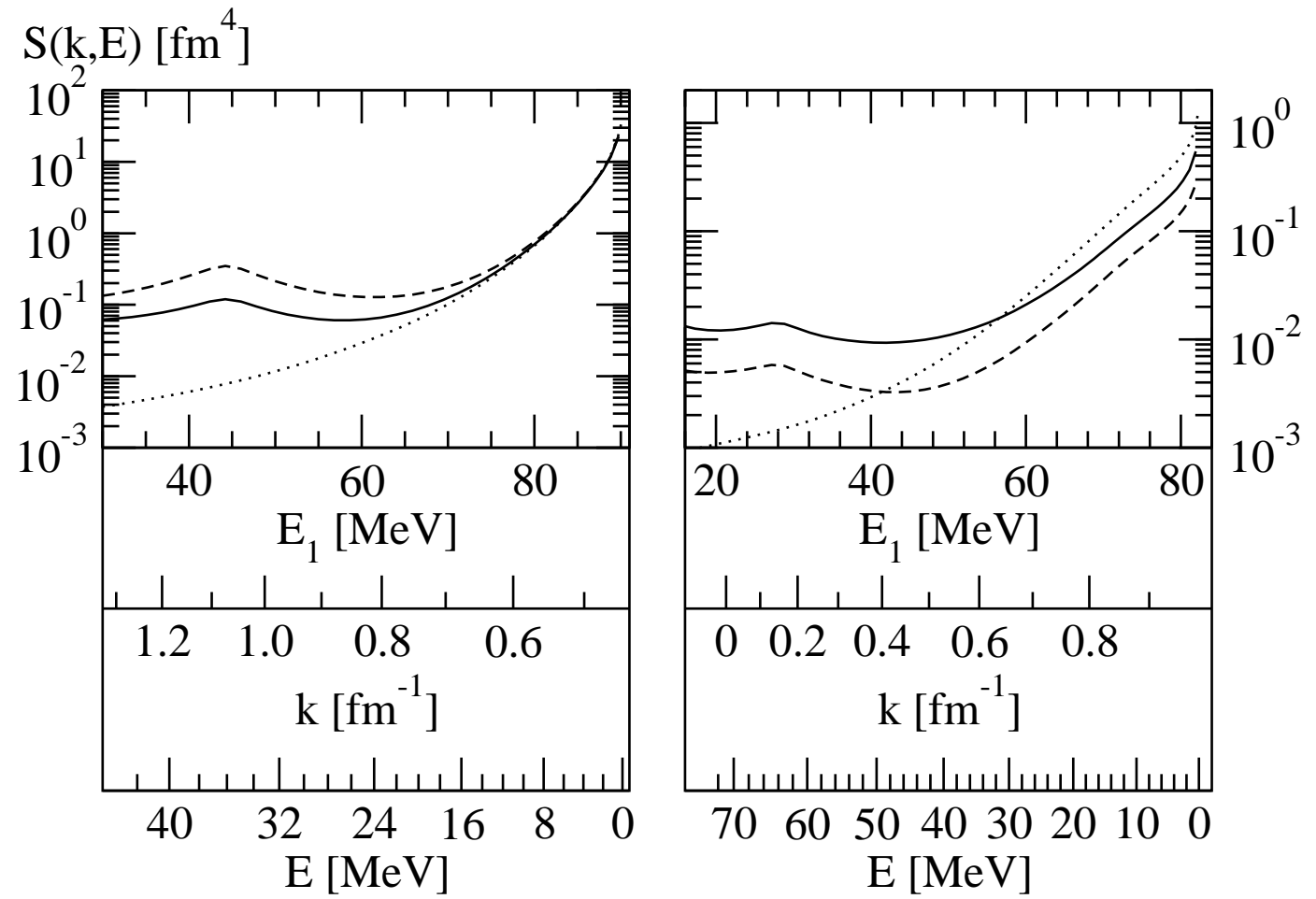

FIG. 79: The spectral function $S(k, E)$ for the proton knockout (dotted line) for two fixed $(\omega-|\vec{Q}|)$ pairs: $\omega=100 \mathrm{MeV},|\vec{Q}|=500 \mathrm{MeV} / \mathrm{c}$ (left) and $\omega=100 \mathrm{MeV},|\vec{Q}|=200 \mathrm{MeV} / \mathrm{c}$ (right) as a function of the ejected proton energy $E_{1}$ for the parallel kinematics $\vec{p}_{1} \| \vec{Q}$. The corresponding values of $k$ and $E$ are also indicated. The dashed line is the result based on the full treatment of FSI but neglecting MEC and 3NF effects in the form of Eq. (10) in [190] for the response functions $R_{L}$ and the solid line is the corresponding result for the response functions $R_{T}$.

power is accessible in experiments where unpolarized electrons are scattered on the polarized ${ }^{3}$ He. Due to the symmetry properties only the component of $\vec{A}^{0}$ perpendicular to the electron plane (usually denoted as $A_{y}^{0}$ ) is different from zero. This observable provides direct information on the importance of FSI because it vanishes for calculations neglecting totally (PWIA and PWIAS) or partly (FSI23) the final state interactions among the three outgoing nucleons.

$A_{y}{ }^{0}$ was measured at MAMI [195] and this experiment supplied very interesting insight into the reaction mechanism, even though the experimental conditions required a lot of integrations over the relevant parts of the phase space. It turned out that at $Q^{2}=0.37$ $(\mathrm{GeV} / \mathrm{c})^{2}$ the analyzing power $A_{y\left(e, e^{\prime} n\right)}^{0}$ results from a coupling of the virtual photon followed 

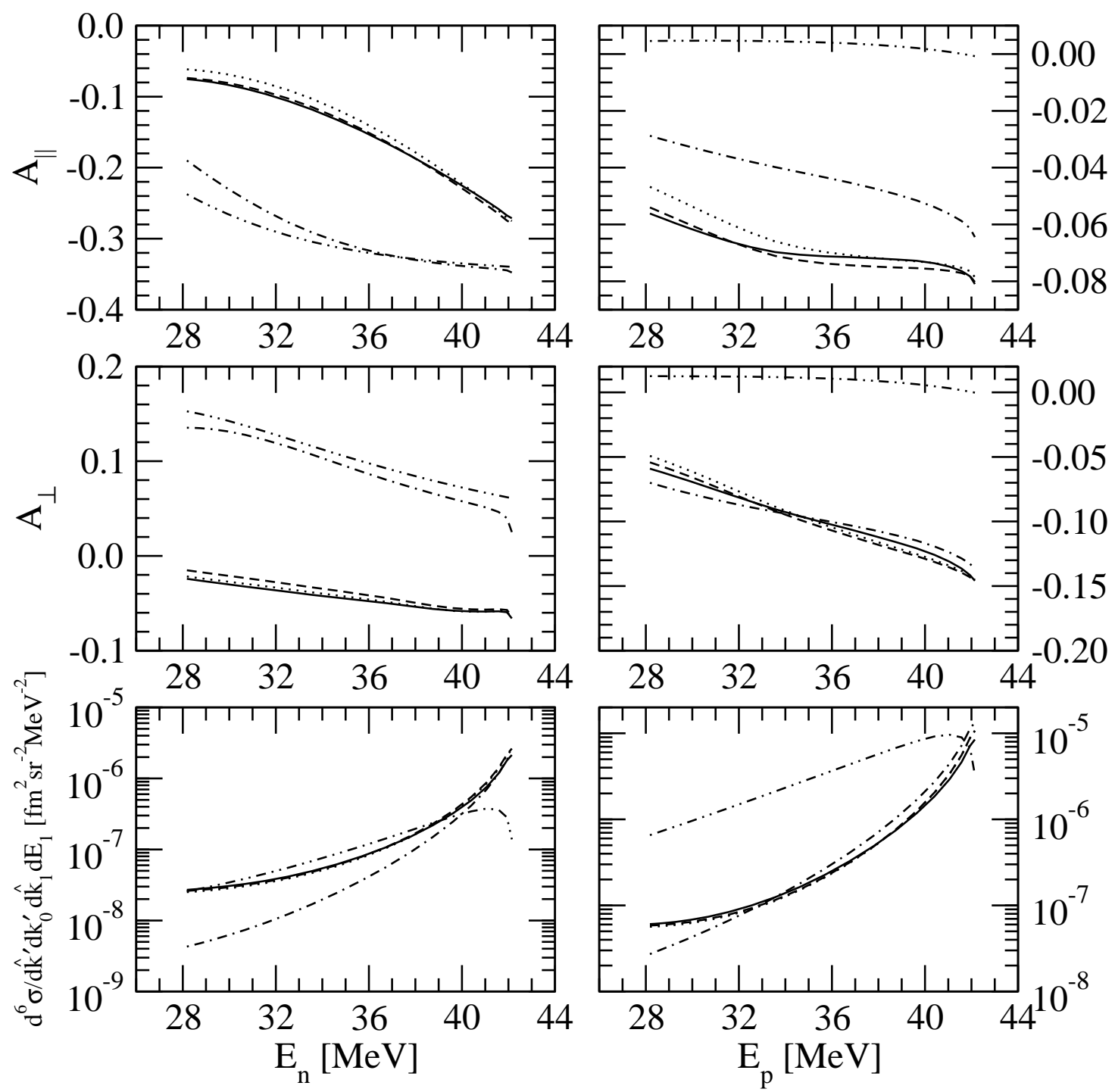

FIG. 80: The dependence of $A_{\|}, A_{\perp}$, and the cross section on the energy of the outgoing neutron (left column) and proton (right column) in the ${ }^{3} \vec{H} e\left(\vec{e}, e^{\prime} N\right) N N$ reaction for $E_{e}=854.5 \mathrm{MeV}, \omega=$ $50 \mathrm{MeV},|\vec{Q}|=300 \mathrm{MeV} / \mathrm{c}$. The double-dot-dashed, dot-dashed, dotted, dashed, and solid curves are based on PWIA, FSI23, FSI, FSI+MEC, and FSI+MEC+3NF, respectively.

by proton-neutron rescattering. Also a different sensitivity to MEC for $A_{y\left(e, e^{\prime} n\right)}^{0}$ and $A_{y\left(e, e^{\prime} p\right)}^{0}$ was confirmed in [195]. 

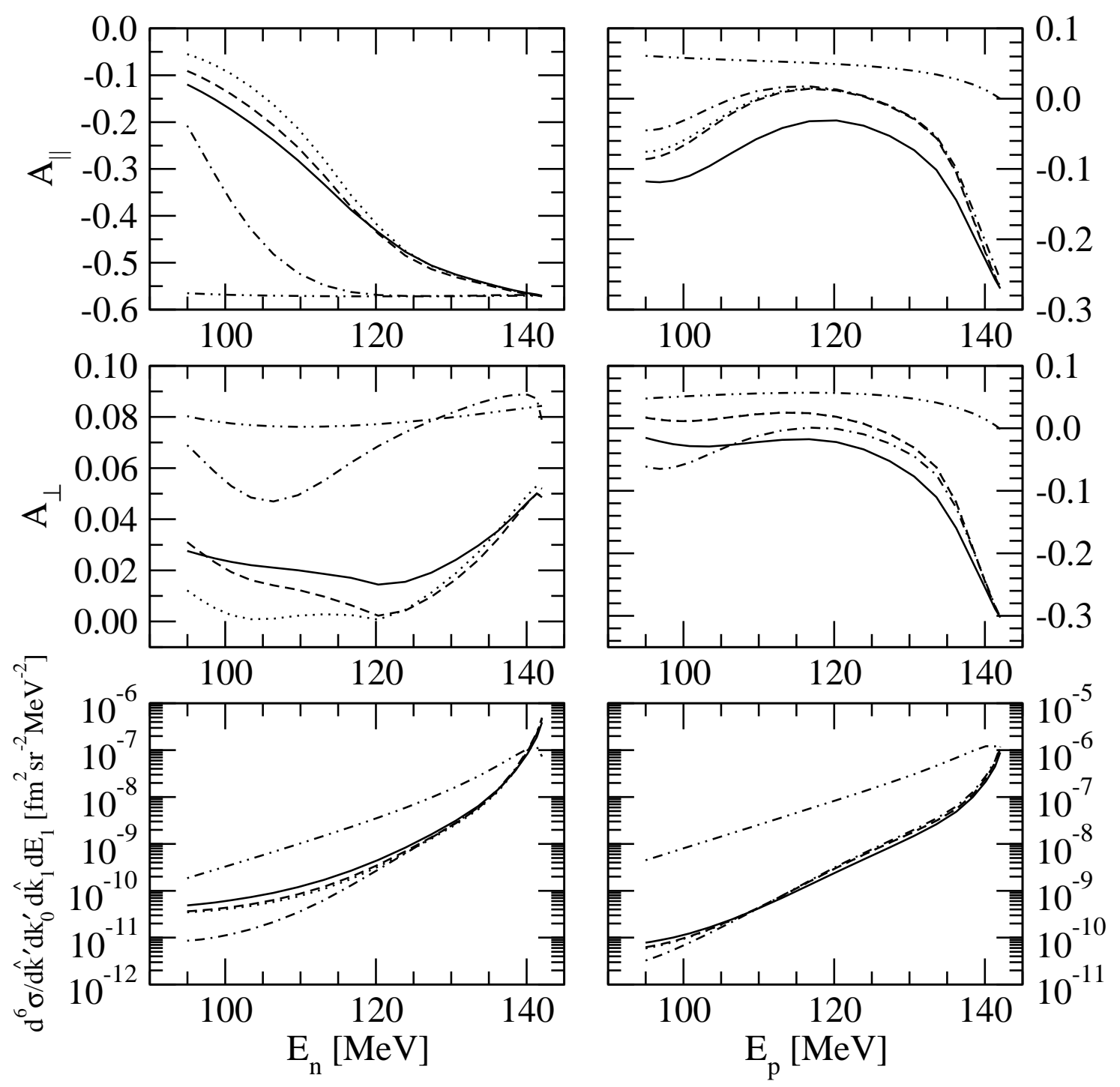

FIG. 81: The same as in Fig. 80 but for $E_{e}=854.5 \mathrm{MeV}, \omega=150 \mathrm{MeV},|\vec{Q}|=500 \mathrm{MeV} / \mathrm{c}$. 


\section{The electron induced complete $3 \mathrm{~N}$ breakup process}

The process ${ }^{3} \mathrm{He}(\mathrm{e}, \mathrm{e}$ 'pp$) \mathrm{n}$ has been measured in the NIKHEF facility [197]. Unfortunately the kinematical conditions were outside of the nonrelativistic domain and the comparison to our theory was generally unsuccessful. Discrepancies up to factors of 4-5 showed up. Possibly the neglecting of the $\Delta$-degrees of freedom was the strongest theoretical defect (see [67]). Such a theoretical analysis requires a close interaction of theory and experiment since the data are taken in a regime far off from the point geometry, where the two protons are detected at fixed angles and fixed energies and this in coincidence with the electron, also detected point-wise. Due to the smallness of the cross section, quite large portions of the phase space have to be covered with large energy and angular bins to arrive at breakup observables with reasonably small error bars. Nevertheless, we would like to show in Figs. 82-85 some examples of eightfold differential cross sections along the kinematical locus for selected breakup configurations. In Fig. 82 three final state interaction peaks are shown where the individual contributions of FSI, MEC and 3NF differ quite strongly from one peak to the other. Quasi free scattering with one final nucleon momentum zero is shown in Fig. 83. Again the individual contributions of the three dynamical ingredients, FSI, MEC and 3NF among each other and against the PWIAS prediction differ significantly. The space star configuration is shown in Fig. 84 for two electron kinematics. Very strong dynamical effects beyond PWIA(S) and FSI23 are seen. Finally cross sections for two electron kinematics are shown in Fig. 85 where two nucleons emerge back to back collinear with the photon momentum $\vec{Q}$.

A second exclusive ${ }^{3} \mathrm{He}(\mathrm{e}, \mathrm{e}$ 'pn $) \mathrm{p}$ experiment was performed at MAMI and is presently analyzed [198]. But again the kinematics is outside of our nonrelativistic domain.

Insight into the NN correlations in a nucleus is an old issue. In a recent measurement [199] an idea proposed also in [200] has been realized. The idea is that the photon is assumed to be absorbed by one nucleon alone, which is knocked out in the direction of the photon. The other two spectator nucleons leave ${ }^{3} \mathrm{He}$ back to back and are assumed not to interact with the knocked out nucleon. This is the same picture as the one underlying the spectral function. But now one regards the fully exclusive process and aims at the relative momentum distribution of the two spectator nucleons. If they did not interact also with each other, one

would see directly the relative momentum distribution of the two nucleons inside ${ }^{3} \mathrm{He}$. In 
our notation using Jacobi momenta this quantity is

$$
C(p)=3 \sum_{m} \sum_{m_{1}, m_{2}, m_{3}}|\Psi(\vec{p}, \vec{q}=0)|^{2}
$$

We investigated that scenario allowing for the complete FSI, for the interaction just among the two spectator nucleons (FSI23), and for the case of no FSI at all and no antisymmetrization in the final state (PWIA). It is easy to see [200] that the only two response functions surviving for parallel kinematics are related to $C(p)$ in PWIA as

$$
\begin{array}{r}
C(p)=\frac{1}{2} \sum_{m} \sum_{m_{1}, m_{2}, m_{3}} R_{L}^{P W I A} / G_{E}^{2} \\
C(p)=\frac{1}{2} \sum_{m} \sum_{m_{1}, m_{2}, m_{3}} 2 m_{N}^{2} R_{T}^{P W I A} /\left(\vec{Q}^{2} G_{M}^{2}\right)
\end{array}
$$

Therefore we investigated $\frac{1}{2} \sum_{m} \sum_{m_{1}, m_{2}, m_{3}} R_{L} /\left(G_{E}\right)^{2}$ and $\frac{1}{2} \sum_{m} \sum_{m_{1}, m_{2}, m_{3}} 2 m_{N}^{2} R_{T} /\left(\vec{Q}^{2} G_{M}^{2}\right)$ as a function of $p$ for different $|\vec{Q}|$-values and for a fixed sequence of the isospin magnetic quantum numbers. In PWIA this is just $C(p)$ and the question is whether, at least with increasing $|\vec{Q}|$, FSI looses importance for this geometry and $C(p)$ can be extracted. It turned out that this does not happen. Interestingly, with increasing $|\vec{Q}|$-values and for proton knockout the FSI23 and FSI predictions approach each other. However, there still remains a noticeable shift toward the result which in addition includes the 3NF's. Thus one has no direct access to $C(p)$. If one is satisfied, however, with a less quantitative result and does not pay attention to the shift caused by that additional $3 \mathrm{NF}$ effect, one has access to a modified $C(p)$ quantity, where the two spectator nucleons, while leaving ${ }^{3} \mathrm{He}$, interact strongly by the NN t-matrix. Therefore since the $t$-matrix is rather well under control one can at least approach the momentum distribution inside ${ }^{3} \mathrm{He}$ modified only by that additional final state interaction. This is illustrated in Fig. 86. Note that this final state interaction leads to a reduction by a factor 10 and more. The curves in Fig. 86 refer to a fixed angle of $90^{\circ}$ between $\vec{p}$ and $\vec{Q}$, but for other angles qualitatively the situation is unchanged. It would be very interesting if these configurations could be measured in our nonrelativistic domain.

In the case when a neutron is knocked out $\frac{1}{2} \sum_{m} \sum_{m_{1}, m_{2}, m_{3}} R_{L} /\left(G_{E}^{n}\right)^{2}$ behaves differently and the FSI23 approximation is unjustified. It is only for $\frac{1}{2} \sum_{m} \sum_{m_{1}, m_{2}, m_{3}} R_{T} /\left(\frac{\vec{Q}^{2}}{2 m_{N}^{2}}\left(G_{M}^{n}\right)^{2}\right)$ that the situation is as favorable as for the proton knockout [200]. This is displayed in Fig. 87. For larger $\mid \vec{Q}$-values $(|\vec{Q}|=600 \mathrm{MeV} / \mathrm{c})$, however, we found that also for $R_{L} /\left(G_{E}^{n}\right)^{2}$ the situation resembles the one for the proton. 
FIG. 82: The eightfold full breakup cross section $d^{8} \sigma /\left(d \hat{k}^{\prime} d k_{0}^{\prime} d \hat{k}_{1} d \hat{k}_{2} d S\right)$ along the arc-length $S$ of the kinematically allowed locus in the $E_{1}-E_{2}$ plane for three different electron configurations: $k_{0}=854.5 \mathrm{MeV}, \vartheta=27.9^{\circ}, k_{0}^{\prime}=750.9 \mathrm{MeV}$ (left), $k_{0}=854.5 \mathrm{MeV}, \vartheta=35.5^{\circ}, k_{0}^{\prime}=754.5 \mathrm{MeV}$ (center) and $k_{0}=854.5 \mathrm{MeV}, \vartheta=35.7^{\circ}, k_{0}^{\prime}=652.3 \mathrm{MeV}$ (right). PWIA (double-dash-dotted line), PWIAS (double-dot-dashed line - overlaps with PWIA), FSI23 (dot-dashed line), FSI (dotted line), FSI+MEC (dashed line) and FSI+MEC+3NF (solid line) predictions are shown. Particles 1 and 2 are protons. The angles of the outgoing nucleons in the system where $\vec{Q} \| \hat{z}\left(\theta_{1}=60.0^{\circ}, \phi_{1}=0.0^{\circ}\right.$, $\theta_{2}=51.0^{\circ}, \phi_{1}=180.0^{\circ}$ (left), $\theta_{1}=60.0^{\circ}, \phi_{1}=0.0^{\circ}, \theta_{2}=34.0^{\circ}, \phi_{1}=180.0^{\circ}$ (center), $\theta_{1}=60.0^{\circ}$, $\phi_{1}=0.0^{\circ}, \theta_{2}=59.0^{\circ}, \phi_{1}=180.0^{\circ}$ (right)) are chosen in such a way that the peaks correspond to the kinematical condition $\vec{k}_{2}=\vec{k}_{3}$ (the final state interaction condition).

FIG. 83: The eightfold full breakup cross section $d^{8} \sigma /\left(d \hat{k}^{\prime} d k_{0}^{\prime} d \hat{k}_{1} d \hat{k}_{2} d S\right)$ along the arc-length $S$ of the kinematically allowed locus in the $E_{1}-E_{2}$ plane for three different electron configurations: $k_{0}=$ 854.5 MeV, $\vartheta=27.9^{\circ}, k_{0}^{\prime}=750.9 \mathrm{MeV}$ (upper row), $k_{0}=854.5 \mathrm{MeV}, \vartheta=35.5^{\circ}, k_{0}^{\prime}=754.5 \mathrm{MeV}$ (middle row) and $k_{0}=854.5 \mathrm{MeV}, \vartheta=35.7^{\circ}, k_{0}^{\prime}=652.3 \mathrm{MeV}$ (lower row). Curves as in Fig. 82. In the left panel particles 1 and 2 are protons while in the right panel particles 1 and 2 are neutron and proton, respectively. The angles of the outgoing nucleons in the system where $\vec{Q} \| \hat{z}\left(\theta_{1}=60.0^{\circ}\right.$, $\phi_{1}=0.0^{\circ}, \theta_{2}=37.0^{\circ}, \phi_{1}=180.0^{\circ}$ (upper row), $\theta_{1}=30.0^{\circ}, \phi_{1}=0.0^{\circ}, \theta_{2}=34.0^{\circ}, \phi_{1}=180.0^{\circ}$ (middle row), $\theta_{1}=60.0^{\circ}, \phi_{1}=0.0^{\circ}, \theta_{2}=49.0^{\circ}, \phi_{1}=180.0^{\circ}$ (lower row)) are chosen in such a way that the quasi free kinematical condition $\vec{k}_{3}=0$ is fulfilled for one central point on the locus. 
FIG. 84: The eightfold full breakup cross section $d^{8} \sigma /\left(d \hat{k}^{\prime} d k_{0}^{\prime} d \hat{k}_{1} d \hat{k}_{2} d S\right)$ along the arc-length $S$ of the kinematically allowed locus in the $E_{1}-E_{2}$ plane for two different electron configurations: $k_{0}=$ 854.5 MeV, $\vartheta=27.9^{\circ}, k_{0}^{\prime}=750.9 \mathrm{MeV}$ (left), and $k_{0}=854.5 \mathrm{MeV}, \vartheta=35.7^{\circ}, k_{0}^{\prime}=652.3 \mathrm{MeV}$ (right). Curves as in Fig. 82. Particles 1 and 2 are protons. The angles of the outgoing nucleons in the system where $\vec{Q} \| \hat{z}\left(\theta_{1}=57.0^{\circ}, \phi_{1}=0.0^{\circ}, \theta_{2}=57.0^{\circ}, \phi_{1}=120.0^{\circ}\right.$ (left), $\theta_{1}=61.5^{\circ}, \phi_{1}=0.0^{\circ}, \theta_{2}=$ $61.5^{\circ}, \phi_{1}=120.0^{\circ}$ (right)) are chosen in such a way that in the c.m. system all particles momenta are equal and form the so called "Mercedes star" in a plane perpendicular to $\vec{Q}$ for one point on the locus (the space-star kinematical condition).

FIG. 85: The eightfold full breakup cross section $d^{8} \sigma /\left(d \hat{k}^{\prime} d k_{0}^{\prime} d \hat{k}_{1} d \hat{k}_{2} d S\right)$ along the arc-length $S$ of the kinematically allowed locus in the $E_{1}-E_{2}$ plane for two different electron configurations: $k_{0}=$ 854.5 MeV, $\vartheta=27.9^{\circ}, k_{0}^{\prime}=750.9 \mathrm{MeV}$ (left), and $k_{0}=854.5 \mathrm{MeV}, \vartheta=35.5^{\circ}, k_{0}^{\prime}=754.5 \mathrm{MeV}$ (right). Curves as in Fig. 82. Particles 1 and 2 are protons. The momentum of particle 1 is parallel to $\vec{Q}$ and the momentum of particle 2 is anti-parallel to $\vec{Q}$.
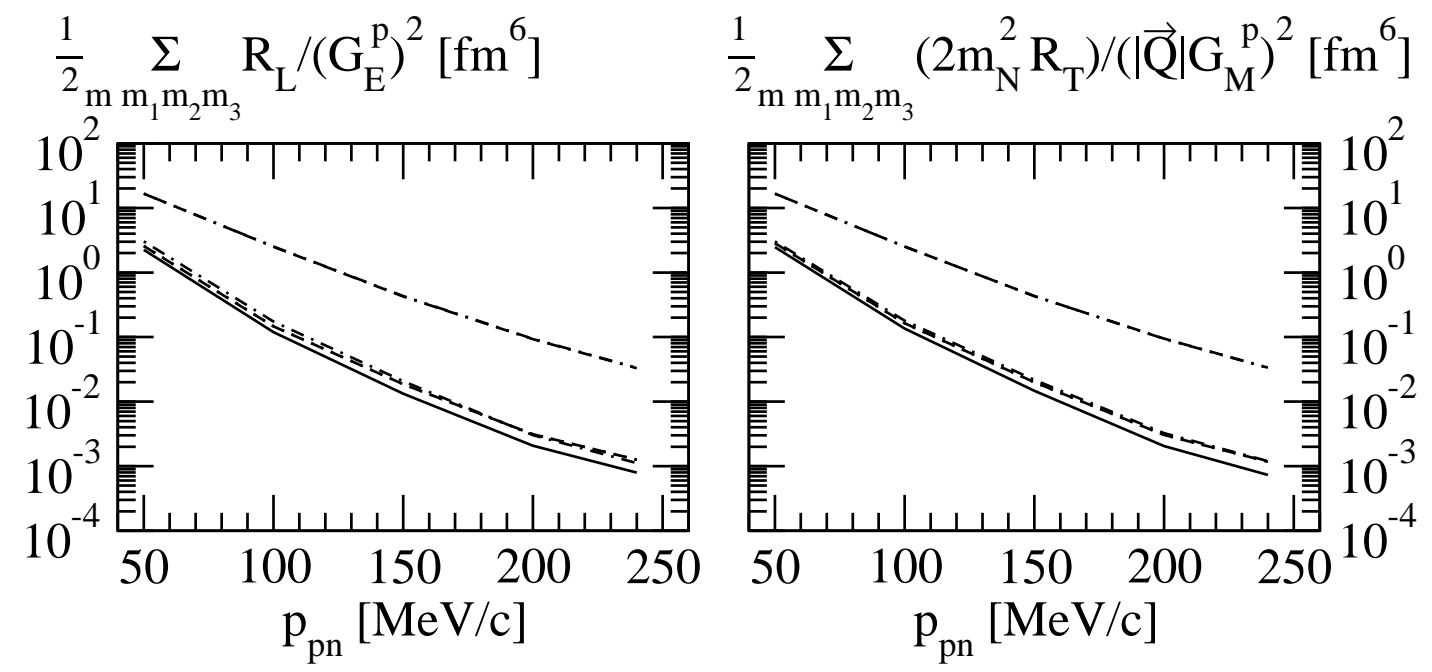

FIG. 86: The quantities from (278) in the case of proton knockout for different dynamical assumptions for $|\vec{Q}|=500 \mathrm{MeV} / \mathrm{c}$ as functions of the relative momentum $p_{p n}$ in the spectator protonneutron subsystem. For the description of the curves see Fig. 80. 

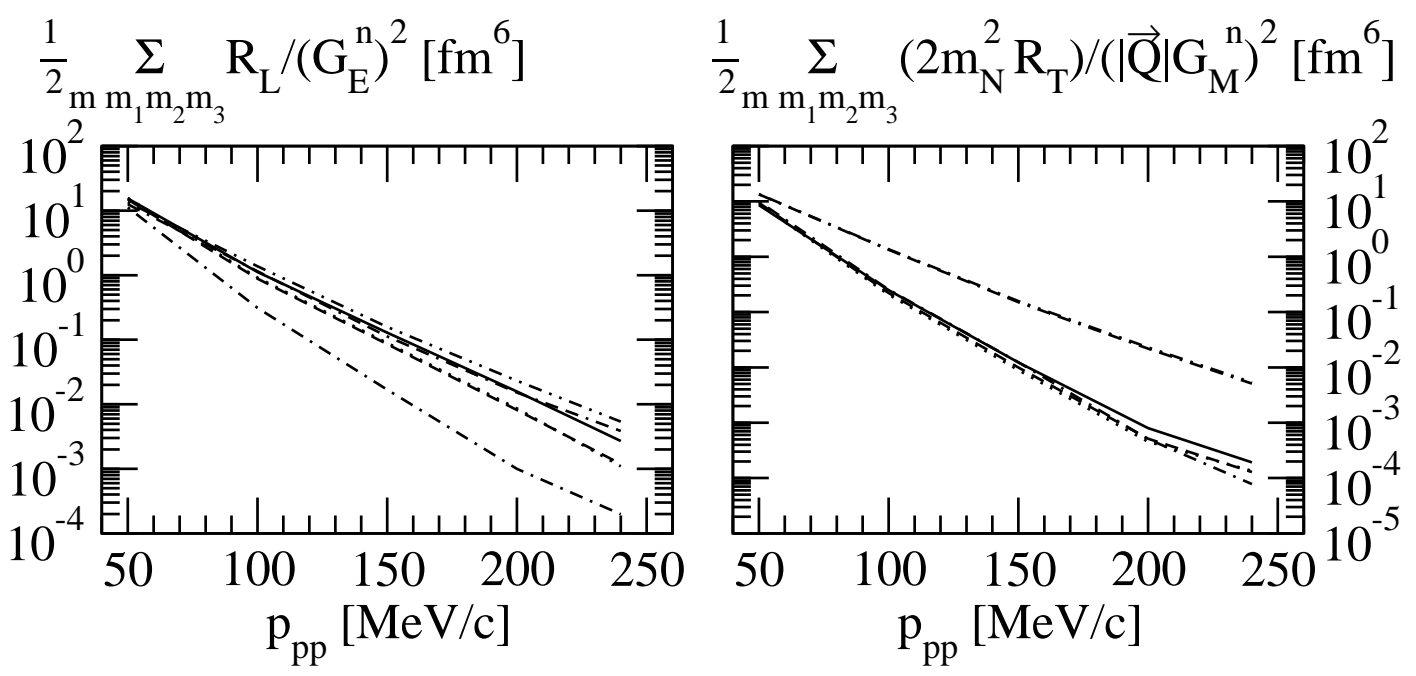

FIG. 87: The quantities from (278) in the case of neutron knockout for different dynamical assumptions for $|\vec{Q}|=500 \mathrm{MeV} / \mathrm{c}$ as functions of the relative momentum $p_{p p}$ in the spectator proton-proton subsystem. Curves as in Fig. 80. 


\section{E. Spin dependent momentum distributions of polarized proton-deuteron clusters} in polarized ${ }^{3} \mathrm{He}$

We address the question whether momentum distributions of polarized $\vec{d} \vec{p}$ clusters in spin oriented ${ }^{3} \mathrm{He}$ are accessible through the ${ }^{3} \mathrm{He}\left(e, e^{\prime} \vec{p}\right) d$ or ${ }^{3} \mathrm{He}\left(e, e^{\prime} \vec{d}\right) p$ processes. Optimal kinematical conditions are that the polarization of ${ }^{3} \mathrm{He}$ and the polarization of the knocked out proton (deuteron) together with the momenta $\vec{k}_{p}$ and $\vec{k}_{d}$ of the final proton and deuteron are collinear to the photon momentum. The spin dependent momentum distribution of proton-deuteron clusters inside ${ }^{3} \mathrm{He}$ is defined as

$$
\mathcal{Y}\left(m, m_{d}, m_{p} ; \vec{q}_{0}\right) \equiv\left\langle\Psi m|| \phi_{d} m_{d}\right\rangle\left|\vec{q}_{0} \frac{1}{2} m_{p}\right\rangle\left\langle\vec{q}_{0} \frac{1}{2} m_{p}\right|\left\langle\phi_{d} m_{d}|| \Psi m\right\rangle
$$

where $\vec{q}_{0}$ is the c.m. proton momentum (the deuteron momentum is $-\vec{q}_{0}$ ) and $m_{p}, m_{d}$, and $m$ are spin magnetic quantum numbers for the proton, deuteron, and ${ }^{3} \mathrm{He}$. This can be expressed as [201]

$$
\begin{aligned}
\mathcal{Y}\left(m, m_{d}, m_{p} ; \vec{q}_{0}\right)= & \mid \sum_{\lambda=0,2} Y_{\lambda, m-m_{d}-m_{p}}\left(\hat{q}_{0}\right) C\left(1 I_{\lambda} \frac{1}{2} ; m_{d}, m-m_{d}, m\right) \\
& \left.C\left(\lambda \frac{1}{2} I_{\lambda} ; m-m_{d}-m_{p}, m_{p}, m-m_{d}\right) H_{\lambda}\left(q_{0}\right)\right|^{2},
\end{aligned}
$$

in terms of the auxiliary quantity $H_{\lambda}\left(q_{0}\right)$

$$
H_{\lambda}\left(q_{0}\right) \equiv \sum_{l=0,2} \int_{0}^{\infty} d p p^{2} \phi_{l}(p)\left\langle p q_{0} \alpha_{l \lambda} \mid \Psi\right\rangle, \lambda=0,2 .
$$

Here $\lambda$ is the relative orbital angular momentum of the proton with respect to the deuteron inside ${ }^{3} \mathrm{He} . \phi_{l}(p)$ and $\langle p q \alpha \mid \Psi\rangle$ are wave function components of the deuteron and ${ }^{3} \mathrm{He}$, respectively. Thus the dependence on the direction $\hat{q}_{0}$ and the magnetic quantum numbers is nicely separated.

We display $H_{\lambda}\left(q_{0}\right)$ in Fig. 88. This shows that $\lambda=0$ dominates the momentum distribution $\mathcal{Y}$ for small relative angular momenta and $H_{0}$ has a node around $q_{0}=400 \mathrm{MeV} / \mathrm{c}$. Near and above that value the $s$ - and $d$-wave contributions are comparable. The momentum distribution itself is shown in Fig. 89 for the case that $\hat{q}_{0}$ points into the direction of the spin quantization axis and ${ }^{3} \mathrm{He}$ is polarized with $m=\frac{1}{2}$. The polarizations of the proton and the deuteron are chosen as $m_{d}=0, m_{p}=\frac{1}{2}$ and $m_{d}=1, m_{p}=-\frac{1}{2}$, respectively. We see an interesting shift in the minima from $q_{0}=300$ to $q_{0}=500 \mathrm{MeV} / \mathrm{c}$, if the polarization of 
the proton (deuteron) switches from a parallel (perpendicular) to an anti-parallel (parallel) orientation in relation to the spin direction of ${ }^{3} \mathrm{He}$. It is easily worked out [201] that the two momentum distributions shown in Fig. 89 coincide in PWIA with the functions $\hat{R}_{L} \equiv$ $R_{L} /\left(G_{E}^{p}\right)^{2}$ and $\hat{R}_{T} \equiv R_{T} /\left(\frac{\vec{Q}^{2}}{2 m_{N}^{2}}\left(G_{M}^{p}\right)^{2}\right)$, when one fixes the spin projections corresponding to the two combinations and chooses the deuteron lab momentum $p_{d}=q_{0}$. In [201] we investigated these two quantities allowing for the complete FSI (without and with 3NF's), for antisymmetrization, and for the inclusion of MEC's as a function of increasing $|\vec{Q}|$. The question is whether they approach the two momentum distributions. The results are quite intricate within the range of $|\vec{Q}|$-values we took into account $(|\vec{Q}| \leq 800 \mathrm{MeV} / \mathrm{c})$. We show in Fig. $90 \hat{R}_{L}$ and in Fig. $91 \hat{R}_{T}$ in comparison to the PWIA results, which are directly the momentum distributions for the two magnetic quantum number combinations. This illustration refers to two deuteron momenta $p_{d}=200$ and $600 \mathrm{MeV} / \mathrm{c}$. For $p_{d}=200$ $\mathrm{MeV} / \mathrm{c} \hat{R}_{L}$ and $\hat{R}_{T}$ have a tendency to approach $\mathcal{Y}\left(m, m_{d}, m_{p} ; \vec{q}_{0}\right)$ within our momentum range $|\vec{Q}|$, but the $3 \mathrm{NF}$ effects are quite noticeable. For $p_{d}=600 \mathrm{MeV} / \mathrm{c}$, however, this is not the case. It turned out when looking into several $p_{d}$-values that the two momentum distributions could be accessed in our restricted $|\vec{Q}|$-range at its upper end only for very small deuteron momenta. For the higher deuteron momenta the FSI and MEC effects precluded that approach. We refer to [201] for a more detailed discussion. The measurement of that polarized setup would be very interesting since all the dynamics comes into the play. 


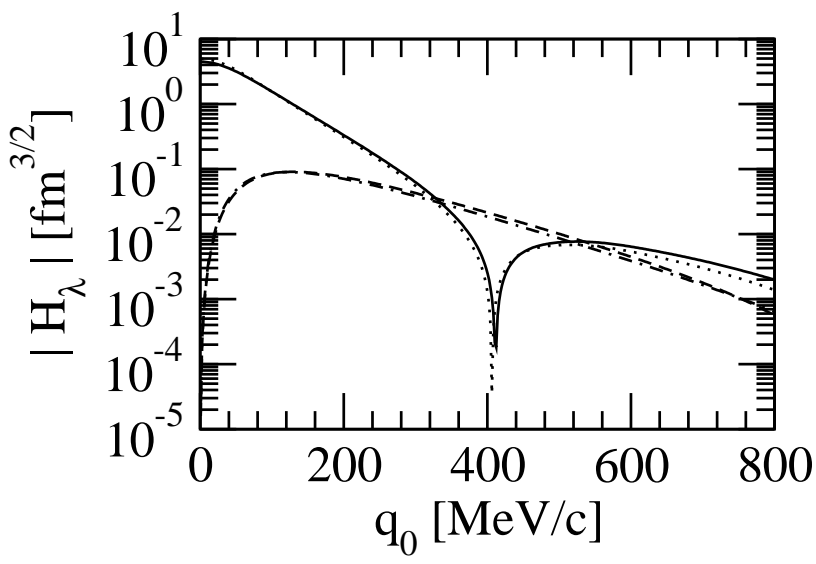

FIG. 88: Absolute value of $H_{\lambda}\left(q_{0}\right)$ defined in (281) for $\lambda=0$ (solid) and $\lambda=2$ (dashed) calculated with the ${ }^{3} \mathrm{He}$ bound state including the UrbanaIX $3 \mathrm{~N}$ force. Corresponding curves neglecting $3 \mathrm{~N}$ force effects (dotted for $\lambda=0$ and dot-dashed for $\lambda=2$ ) are also shown. Note $H_{0}\left(q_{0}\right)<0$ for $q_{0}>$ $400 \mathrm{MeV} / \mathrm{c}$, while $H_{2}\left(q_{0}\right)$ remains always positive for the shown $q_{0}$-values.

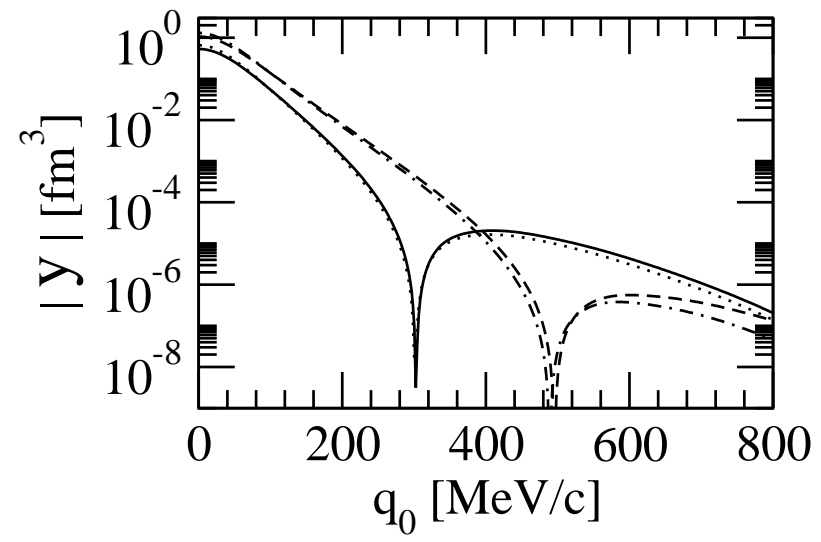

FIG. 89: Spin-dependent momentum distributions $\mathcal{Y}\left(m=\frac{1}{2}, m_{d}=0, m_{p}=\frac{1}{2} ;\left|\overrightarrow{q_{0}}\right| \hat{z}\right)$ (solid line) and $\mathcal{Y}\left(m=\frac{1}{2}, m_{d}=1, m_{p}=-\frac{1}{2} ;\left|\vec{q}_{0}\right| \hat{z}\right)$ (dashed line) defined in (280) for $\vec{p} \vec{d}$ clusters in ${ }^{3}$ He when UrbanaIX $3 \mathrm{NF}$ is included. Corresponding curves neglecting $3 \mathrm{~N}$ force effects are: dotted for $m_{p}=\frac{1}{2}$ and dot-dashed for $m_{p}=-\frac{1}{2}$ ). 

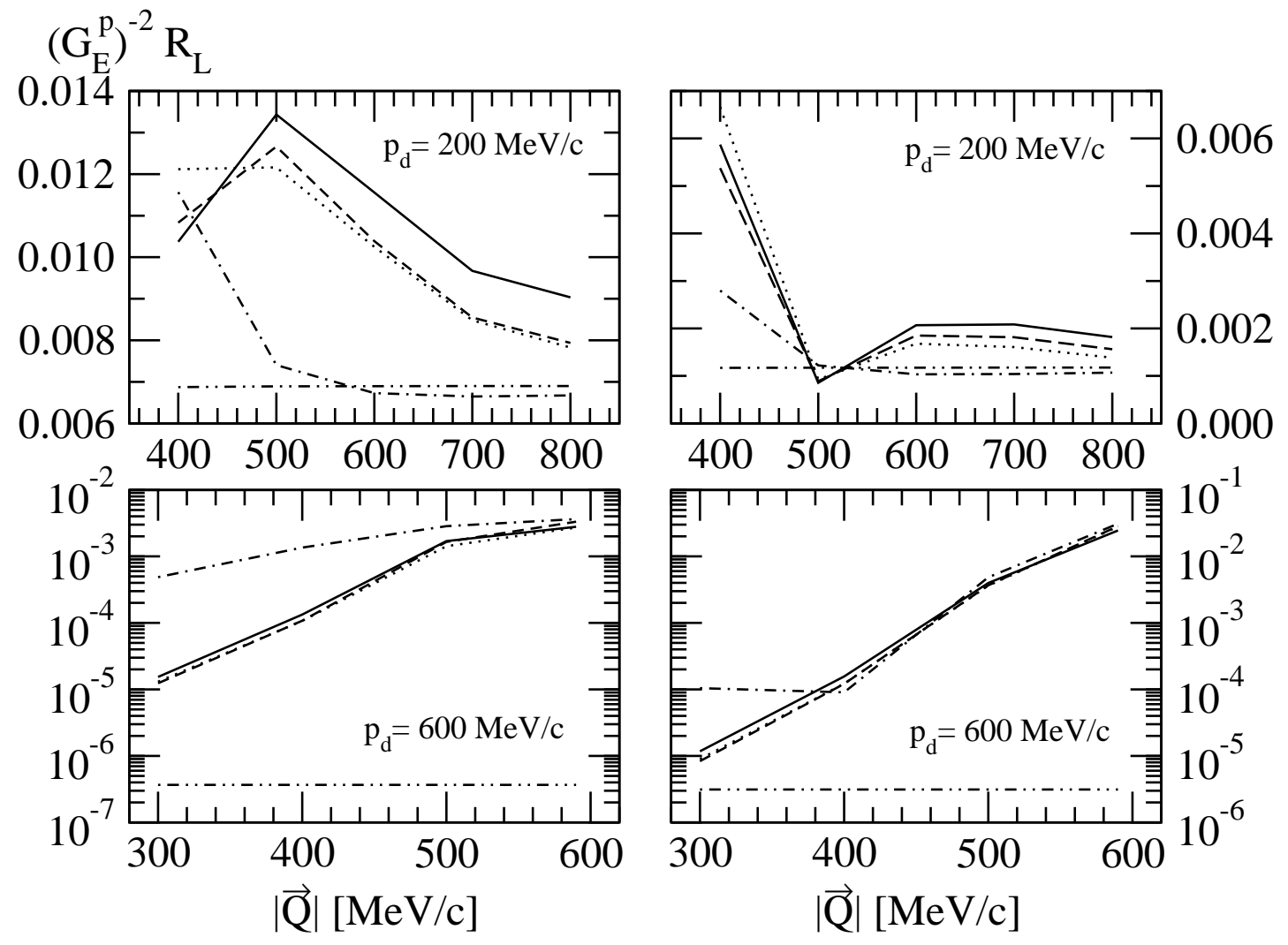

FIG. 90: $\frac{1}{\left(G_{E}^{p}\right)^{2}} R_{L}$ as a function of the three-momentum transfer $|\vec{Q}|$ for $p_{d}=200 \mathrm{MeV} / \mathrm{c}$ (upper row) and $p_{d}=600 \mathrm{MeV} / \mathrm{c}$ (lower row). Two left panel figures are for the $m=\frac{1}{2}, m_{d}=1, m_{p}=-\frac{1}{2}$ and two right panel figures for the $m=\frac{1}{2}, m_{d}=0, m_{p}=\frac{1}{2}$ combination of the spin magnetic quantum numbers. The curves correspond to the PWIA (double-dot-dashed), PWIAS (dot-dashed), FSI (dotted), FSI+MEC (dashed) and FSI+MEC+3NF (solid) results. 

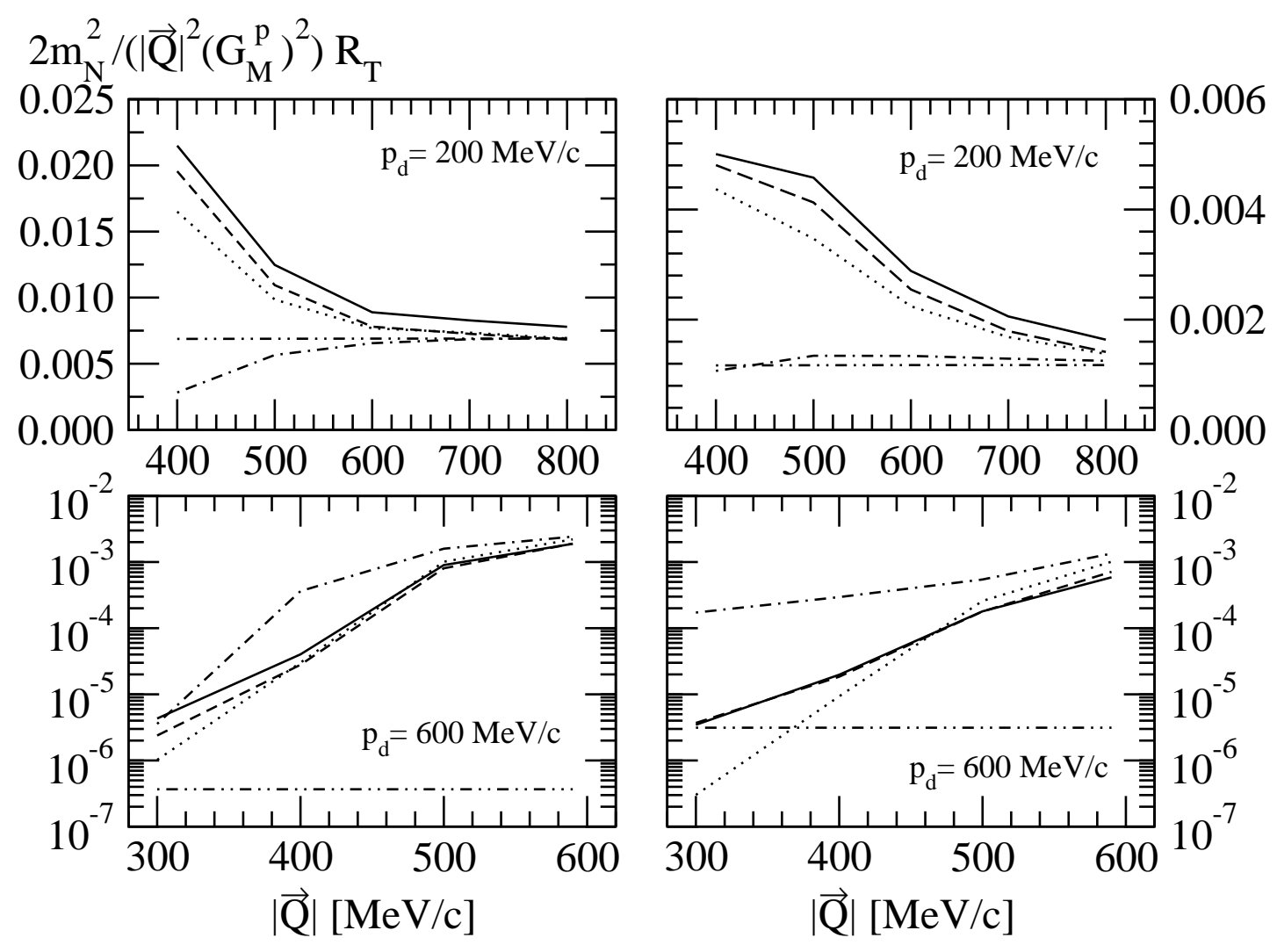

FIG. 91: The same as in Fig. 90 but for $\frac{2 m_{N}^{2}}{|\vec{Q}|^{2}\left(G_{M}^{p}\right)^{2}} R_{T}$. 


\section{F. $3 \mathrm{~N}$ Photodisintegration of ${ }^{3} \mathrm{He}$}

The semiexclusive ${ }^{3} \mathrm{He}(\gamma, N) N N$ reaction where only one nucleon is detected appears to be rather easily accessible. We show in Figs. 92-94 the energy spectra of the outgoing nucleon at several nucleon emission lab angles. The structures for proton and neutron emissions are quite different. While the structures for $\mathrm{E}_{\gamma}=12$ and $40 \mathrm{MeV}$ are similar there is a quite noticeable change in shapes when going to $\mathrm{E}_{\gamma}=120 \mathrm{MeV}$. The $3 \mathrm{NF}$ effects in the predictions with explicit MEC's are relatively small. Due to the semiexclusive character they are washed out in relation to rather significant effects in the exclusive processes discussed below. Also the Siegert approach including the $3 \mathrm{NF}$ is shown and it deviates, especially at $120 \mathrm{MeV}$, from the explicit MEC predictions. We refer to [202] for discussions and insights into the complex underlying interplays. In any case MEC effects are very strong at the two higher energies and measurements would be very rewarding to test the theoretical predictions.

FIG. 92: The semiexclusive ${ }^{3} \mathrm{He}(\gamma, \mathrm{N}) \mathrm{NN}$ processes for neutron or proton emissions at various lab angles and $\mathrm{E}_{\gamma}=12 \mathrm{MeV}$. The solid curve corresponds to MEC+AV18+UrbanaIX dynamics, the dashed curve to MEC+AV18, the dotted curve to Siegert+AV18+UrbanaIX and the dot-dashed curve to AV18 with the single nucleon current only.

FIG. 93: The same as in Fig. 92 but at $\mathrm{E}_{\gamma}=40 \mathrm{MeV}$.

FIG. 94: The same as in Fig. 92 but at $\mathrm{E}_{\gamma}=120 \mathrm{MeV}$.

For the case of semiexclusive reactions with polarized $\gamma$ and/or polarized $\overrightarrow{3 \mathrm{He}}$ we calculated the spin observables of (211) in a range of outgoing nucleon lab angles from $\theta=10^{\circ}$ to $170^{\circ}$. In Figs. 95 and 96 we show our predictions for the nucleon outgoing angle $\theta=90^{\circ}$

at $E_{\gamma}=12 \mathrm{MeV}$ and $E_{\gamma}=40 \mathrm{MeV}$, respectively. The $A_{x}^{\gamma}$ analyzing power is large and its magnitude approaches one at the higher neutron energies. It is practically insensitive to the 
inclusion of the $3 \mathrm{NF}$ both for outgoing neutron and proton. In contrast, the $A_{y}^{3} \mathrm{He}$ analyzing power and spin correlation coefficients $C_{x, y(y, x)}$ exhibit rather large sensitivity to the $3 \mathrm{NF}$ at $E_{\gamma}=12 \mathrm{MeV}$ when the outgoing neutron is measured. The $3 \mathrm{NF}$ effects modify the magnitude of the three observables in a very similar way. Both spin correlation coefficients are quite similar to each other and to $A_{y}^{3} \mathrm{He}$. They are approximately of the same magnitude but of opposite sign to $A_{y}^{3} \mathrm{He}$. The effects of the $3 \mathrm{NF}$ extend over a large energy and angular range of the outgoing neutron and in some cases are as large as $\approx 20 \%$. Similar statements are true when the outgoing proton is measured. In this case, however, the largest 3NF effects appear in the region of high energies of the outgoing proton. At $E_{\gamma}=40 \mathrm{MeV}$ the $3 \mathrm{NF}$ effects are drastically reduced. These results show that it would be very interesting to measure such spin observables.

FIG. 95: The analyzing powers and spin correlation coefficients as a function of the outgoing neutron (left) or proton (right) lab energy for the ${ }^{3} \overrightarrow{H e}(\vec{\gamma}, n) p p\left({ }^{3} \mathrm{He}(\vec{\gamma}, p) p n\right)$ reaction at $E_{\gamma}=$ $12 \mathrm{MeV}$ and $\theta_{l a b}=90^{\circ}$. The dashed curve is the prediction based on MEC+AV18 and the solid on MEC+AV18+UrbanaIX.

FIG. 96: The same as in Fig. 95 but at $E_{\gamma}=40 \mathrm{MeV}$. 
Then we come to the most informative process, the exclusive ${ }^{3} \mathrm{He}(\gamma, p p) n$ reaction. We scanned the full phase space and searched for $3 \mathrm{~N}$ force effects by switching on and off the $3 \mathrm{~N}$ force. To have a quantitative measure we defined

$$
\Delta\left(\Omega_{1}, \Omega_{2}, S\right) \equiv\left|d^{5} \sigma^{\mathrm{NN}+3 \mathrm{NF}}-d^{5} \sigma^{\mathrm{NN}}\right| / d^{5} \sigma^{\mathrm{NN}} \times 100 \%,
$$

where $\Omega_{1}, \Omega_{2}$ are the directions of the two outgoing protons and $S$ is the position on the kinematical locus. In this manner we can associate $\Delta$-values to all regions in phase space. In order to locate phase space regions uniquely, we show three two-dimensional plots. The first one is the $\Theta_{1}-\Theta_{2}$ plane for the two polar angles of the proton detectors. The second one is the $\Theta_{1}-\Phi_{12}$ plane, where $\Phi_{12} \equiv\left|\Phi_{1}-\Phi_{2}\right|$ is the relative azimuthal angle of these two detectors. Finally, the third one is the $\mathrm{E}_{1}-\mathrm{E}_{2}$ plane for the correlated energies of the two detected protons. To fill the three planes we proceed as follows. The whole phase-space is filled with discrete points corresponding to certain grids in $\Theta_{1}, \Theta_{2}, \Phi_{1}, \Phi_{2}$, and $E_{1}$. For $\Theta_{1}$ and $\Theta_{2}$ fixed we search for the maximal value of $\Delta$ in the 3-dimensional subspace spanned by $\Phi_{1}, \Phi_{2}$, and $\mathrm{E}_{1}$. Then we combine those maximal $\Delta$-values into three groups and associate certain grey tones to those group values. Next we choose a fixed $\Theta_{1}$ and $\Phi_{12}=\left|\Phi_{2}\right|$ (one can put $\Phi_{1}=0^{\circ}$ ) and search again for the maximal values of $\Delta$ in the 2-dimensional subspace spanned by $\Theta_{2}$ and $E_{1}$. The same grey tones and groupings are then applied. Finally, in the $\mathrm{E}_{1}-\mathrm{E}_{2}$ plane we search for the maximal $\Delta$-values in the three dimensional subspace spanned by $\Theta_{1}, \Theta_{2}, \Phi_{12}$ and repeat the procedure. For a larger number of groups see [112]. This procedure has been applied and the results are shown in Figs. 97-99. We performed this investigation for three photon lab energies $\mathrm{E}_{\gamma}=12,40$ and $120 \mathrm{MeV}$. The results presented in Fig. 97 are based on AV18+UrbanaIX and the explicit MECs. The choice of the border values for the three groups is of course arbitrary. The group with the largest effects according to those choices appear in dark tone and the group with the smallest effects appear in light tone. The remaining group with $3 \mathrm{NF}$ effects in between is located in the white areas.

As an example let us regard $E_{\gamma}=120 \mathrm{MeV}$. Large $3 \mathrm{NF}$ effects are predicted for instance for the detector angles $\theta_{1}, \theta_{2} \leq 60^{\circ}$ and all relative azimuthal angles $\phi_{12}$. The energies $E_{1}$, $E_{2}$ lie on a kinematical locus and the $3 \mathrm{NF}$ effects are largest as displayed by the dark spots. In addition there are smaller regions like $\theta_{1}$ as before but $\theta_{2} \approx 180^{\circ}$.

In order to plan experiments in the future the absolute values of the fivefold differential cross sections are important. Therefore we show those values in Fig. 98 again arranged in 
FIG. 97: The $3 \mathrm{NF}$ effects spread over the full $3 \mathrm{~N}$ breakup phase-space. It is mapped into the $\Theta_{1}-\Theta_{2}, \Theta_{1}-\Phi_{12}$ and the $E_{1}-E_{2}$ planes. The three rows refer to the three photon lab energies $E_{\gamma}=12,40$ and $120 \mathrm{MeV}$. Regions where the effects are largest are shown in dark and regions with smallest effects in light tone. In the white regions the effects lie between the two border values given to the right of each row. These results are based on AV18+MEC and AV18+UrbanaIX+MEC predictions.

FIG. 98: The distribution of the magnitudes of the cross sections over the full $3 \mathrm{~N}$ breakup phasespace for the three $\gamma$ energies as in Fig. 97. Now the white areas belong to the smallest cross section values and the light and dark tone regions to the cross section values as indicated on the right for each row.

FIG. 99: The regions in the $3 \mathrm{~N}$ phase-space where the breakup cross sections for $E_{\gamma}=120 \mathrm{MeV}$ are larger than $0.1 \mu \mathrm{b} \mathrm{sr}^{-2} \mathrm{MeV}^{-1}$, the $3 \mathrm{NF}$ effects are larger than $20 \%$, and the choice of the two-body current between MEC and Siegert causes effects not greater than $10 \%$.

three groups. Here the white area refers to the smallest cross section values. It is easily seen investigating the kinematics, that the configurations corresponding to the darkest group are of the type of FSI or close to it. We show two examples in Figs. 102-103.

Finally, we locate the regions in phase space for $E_{\gamma}=120 \mathrm{MeV}$ where the cross sections are measurable (larger than $0.1 \mu \mathrm{b} \mathrm{sr}^{-2} \mathrm{MeV}^{-1}$ ) and the $3 \mathrm{NF}$ effects are larger than $20 \%$. Despite the fact that our Siegert approach is less suited for $E_{\gamma}=120 \mathrm{MeV}$ we also performed calculations and added the further condition, that the two choices of currents, explicit MEC or Siegert, deviate at most by $10 \%$. This selects configurations which are dominated by $3 \mathrm{NF}$ effects and to a smaller extent by the choice of the current (among the ones we had at our disposal). Those small groups of configurations in phase space are displayed in Fig.99.

Now we show some configurations for fixed angles along the S-curve displaying different 
situations. In Fig. 100 we see large two-body current and some 3NF effects. In contrast in Fig. 101 only very small 3NF effects appear. Finally in Figs. 102 and 103 large 3NF effects show up in FSI peaks. That variety of current and $3 \mathrm{NF}$ effects would be a fruitful and detailed source of information on the dynamics and an experimental investigation appears very worthwhile.

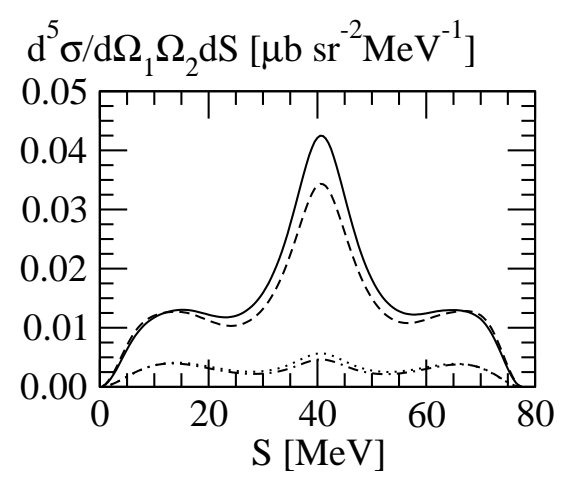

FIG. 100: Fivefold differential cross sections for the angular configuration $\Theta_{1}=88^{\circ}, \Phi_{1}=0^{\circ}$, $\Theta_{2}=100^{\circ}, \Phi_{2}=11^{\circ}$, at photon lab energy $\mathrm{E}_{\gamma}=120 \mathrm{MeV}$. The AV18 predictions with single-nucleon current and with single nucleon current + MEC are given by the dot-dashed and dashed curves, respectively. The corresponding AV18+MEC+UrbanaIX predictions are given by the dotted and solid curves, respectively.

In actual experiments one is far away from our point geometry results and a certain amount of integration over angular regions and energy intervals has to be accepted. As an illustration we regard the two peaks in Fig. 103, which in point geometry exhibit 3NF effects of $\approx 20 \%(\approx 23 \%)$ for the left (right) peak. Will they survive if the cross sections will be summed up over certain angular and energy regions? To that aim we integrated the cross sections over all four angles and single nucleon energy, allowing for deviations up to $5^{\circ}$ around the central values for the angles and $5 \mathrm{MeV}$ in one of the single nucleon energies, $E_{1}$, where the $\mathrm{S}$-curve in Fig. 103 is related to a kinematical locus in the $\mathrm{E}_{1}-\mathrm{E}_{2}$ plane. This summation is repeated replacing $5^{\circ}$ in angles and $5 \mathrm{MeV}$ in energy by $10^{\circ}$ in angles and $10 \mathrm{MeV}$ in energy. The resulting cross section values are displayed in Table III without and with $3 \mathrm{NF}$. Their ratios around 1.20 show still a significant effect. From those cross section values as well as from the magnitude of the effects an experimental realization appears feasible [203]. 


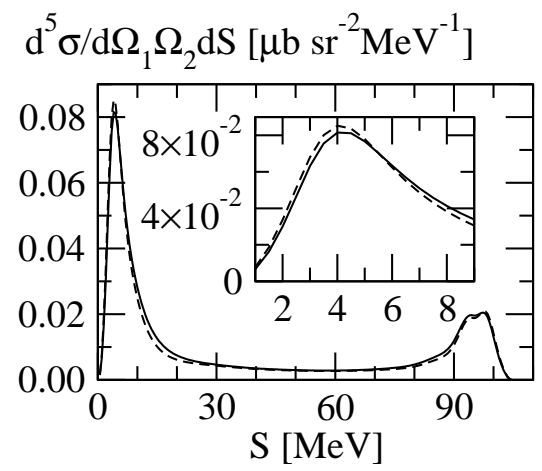

FIG. 101: Fivefold differential cross sections for the angular configuration $\Theta_{1}=30^{\circ}, \Phi_{1}=0^{\circ}$, $\Theta_{2}=145^{\circ}, \Phi_{2}=77^{\circ}$, at photon lab energy $\mathrm{E}_{\gamma}=120 \mathrm{MeV}$. The AV18+MEC predictions are given by the dashed curve and the corresponding AV18+UrbanaIX predictions are given by the solid curve.

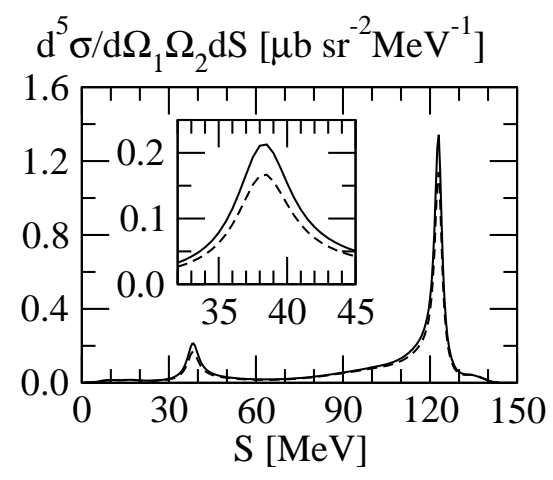

FIG. 102: The same as in Fig. 101 but for $\Theta_{1}=142^{\circ}, \Phi_{1}=0^{\circ}, \Theta_{2}=27^{\circ}, \Phi_{2}=180^{\circ}$.

Note that in [112] the NN interaction was taken in the form of the $n p$-interaction only, while in the present work and in [202] we include $p p$ and $n n$ interactions by the " $\frac{2}{3}+\frac{1}{3}$ " rule $[110,111]$. We refer to [202] and [112] where several additional investigations are displayed.

Often in the literature photodisintegration is treated by keeping only the lowest multipole E1. This extreme low energy assumption would be quite insufficient for nearly all phase space regions and for all three photon energies studied in this paper. This can again be quantified and we find, that even at $12 \mathrm{MeV}$ there are plenty of breakup configurations where the electric multipole E1 alone would deviate by more than $20 \%$ from the result when 


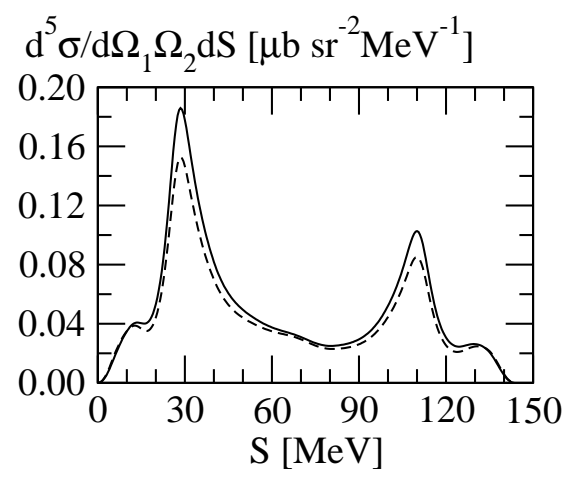

FIG. 103: The same as in Fig. 101 but for $\Theta_{1}=70^{\circ}, \Phi_{1}=0^{\circ}, \Theta_{2}=100^{\circ}, \Phi_{2}=180^{\circ}$.

\begin{tabular}{l|c|c|c|c|c}
\hline $\mathrm{S}=110 \mathrm{MeV}$ & choice I & choice II & $\mathrm{S}=30 \mathrm{MeV}$ & choice I & choice II \\
\hline without 3NF & $0.683 \mathrm{E}-07$ & $0.138 \mathrm{E}-05$ & without 3NF & $0.234 \mathrm{E}-06$ & $0.386 \mathrm{E}-05$ \\
with 3NF & $0.824 \mathrm{E}-07$ & $0.166 \mathrm{E}-05$ & with 3NF & $0.280 \mathrm{E}-06$ & $0.451 \mathrm{E}-05$ \\
\hline ratio & 1.21 & 1.20 & ratio & 1.20 & 1.17
\end{tabular}

TABLE III: Integrated cross sections $\Delta \sigma$ (in $\mathrm{fm}^{2}$ ) at $E_{\gamma}=120 \mathrm{MeV}$ without and with $3 \mathrm{NF}$ for the two choices of integration ranges (see text). This refers to the two peaks in Fig. 103 around $S=110$ and $30 \mathrm{MeV}$. The ratios are practically as large as for point geometry.

all multipoles are included. Again for detailed plots see [112, 202]. 


\section{ADDENDUM}

We would like to add brief remarks on several issues also relevant in the $3 \mathrm{~N}$ system and which have not been addressed in this review: relativistic approaches, y-scaling and weak processes. These remarks will mostly serve to provide recent references.

The covariant spectator theory includes relativity in a manifestly covariant way. It restricts all but one of the particles to their mass shell, which leads to the technically welcome property that all loop integrations are three-dimensional. Also the manifest covariance goes with the property that all boosts are kinematic and the off-shell particle has negative energy components. Cluster separability holds which in a Hamiltonian approach has been formally solved in [204] but presents a big challenge in the practical application. The spectator equations have been applied to the NN system including electromagnetic processes as well as to the $3 \mathrm{~N}$ bound state. Most recently a complete Feynman diagram expansion for the electromagnetic form factors and the three-body photo- and electro-disintegration of the three-body bound state has been derived. For the long list of references see the most recent ones $[205,206]$

Another approach including relativity is the relativistic Hamiltonian dynamics. The seminal paper is by E.P. Wigner [207]. It lays the ground for the physical requirements of special relativity in quantum mechanics leading to the necessary and sufficient conditions for the existence of an unitary (ray) representation of the inhomogeneous Lorentz group (Poincare group) in the quantum mechanical Hilbert space. Further seminal papers are by P.A.M. Dirac [208] who introduced in the Hamiltonian formulation the "point","instant" and "front" forms of dynamics. B. Bakamjian and L.H. Thomas [209] constructed the first relativistic quantum mechanical model of two interacting particles in Dirac's "instant" form of dynamics. L. Foldy [210] pointed to the importance of macroscopic locality (cluster separability). F. Coester [211] extended the work by Bakamjian and Thomas to systems of three particles with a scattering operator consistent with the principle of macroscopic locality. Finally S.N. Sokolov [212] generalized relativistic Hamiltonian dynamics to N particles under the condition of macroscopic locality. Motivated by Sokolov's work F. Coester and W.N. Polyzou [204] treated cluster properties for any fixed number of particles in the instant-, front- and point-forms of the dynamics. The review of relativistic Hamiltonian dynamics by B.D. Keister and W.N. Polyzou [213] includes in addition to basic concepts the material 
specific for the three-body problem, on how to treat spin, and on matrix elements of tensor and spin operators (currents). The most general treatment of the two-body problem in relativistic dynamics appeared in [214]. It is not limited to Diracs forms of dynamics. They are replaced by representations of Poincare Clebsch Gordon coefficients. Special choices lead to Diracs forms. This approach was generalized in [215] to many bodies. Particle production was included in [216]. For relativistic variational Monte Carlo calculations of the N-body bound states the paper [217] is suited. The Balian-Brezin method for treating angular momentum reduction in the Faddev equation [218] has been generalized to the relativistic case in [219]. A very basic investigation [220] shows, that given a relativistic Hamiltonian dynamics it is possible to construct a conserved covariant current operator that satisfies cluster properties and which will produce any kind of experimental form factors. In other words, it shows, that Poincare invariance, current covariance and cluster properties do not constrain form factors.

The above citations refer to basic formalisms and we refrain to list the various applications of Hamiltonian dynamics to electron scattering, which are anyhow mostly carried through for hadron form factors. This is outside the scope of this review. We restrict ourselves only to a few recent ones, which provide references to earlier work and to studies by J. Carbonell and collaborators: [221-224]. All that work briefly addressed opens the doors to generalize what has been presented in this review into a relativistic Hamiltonian scheme.

The issue of $\mathbf{y}$-scaling has been nicely discussed in [225] including a rich list of references, among them the seminal work in [226] by G. West and the theoretical investigations based on plane wave impulse approximation by the Rome and Rehovot groups [227, 228], to mention just those two. Under PWIA it can be shown that the cross section in inclusive electron scattering, which depends on $|\vec{Q}|$ and $\omega$, at high momentum transfers, after the cross section has been divided by an appropriately chosen single nucleon cross section, is a function of a single variable y. This y-scaling variable is itself a function of $|\vec{Q}|$ and $\omega$. Of course the question is, whether the underlying assumptions are realized in nature and especially, whether the interaction of the knocked out nucleon with the recoiling system can be neglected or sufficiently well taken into account. That issue has been critically studied in two model investigations, one in a nonrelativistic two-nucleon model [229], and one in a light front formalism of relativistic quantum mechanics [230], where a conserved model hadronic current operator has been used which is covariant with respect to a unitary representation of the 
Poincare group.

Weak processes have been discussed in [26] where also references to previous work can be found. A more recent study [166] evaluated the decay rate for the process $\mu^{-}+{ }^{3} H e \rightarrow{ }^{3} H+\nu_{\mu}$ including angular correlation parameters. The total rate agreed nicely with experiment and showed only a weak model dependence. The two-body currents, which turned out to be significant, could be constrained in the tritium beta decay. This paper also provides some clues on the induced pseudoscalar form factor $G_{p s}$. The process $\mu^{-}+{ }^{3} H e \rightarrow d+n+\nu_{\mu}$ has been studied in [231] using a Faddeev treatment for bound and continuum states. Only the single nucleon current has been employed. Very large effects of the final state interaction have been found, which brought theory into the vicinity of the experimental decay rate $d \Gamma / d E_{d}[232,233]$. 


\section{SUMMARY AND OUTLOOK}

This review has been devoted to electron and photon induced processes in the $3 \mathrm{~N}$ system, restricted to a mostly nonrelativistic kinematical regime. We focused on the Faddeev scheme which for the various processes has been outlaid in some detail. This guarantees rigorous solutions of the $3 \mathrm{~N}$ bound and scattering states for any type of $\mathrm{NN}$ and $3 \mathrm{~N}$ forces. Naturally the electromagnetic currents play a central role, too. Since this issue of current has been dealt with at many places in the literature we were relatively brief and just described the two-body currents which were used in our calculations on top of the standard single nucleon current. These are the dominant $\pi$ - and $\rho$-like currents related to the NN force AV18. Then we provided expressions for the rich set of observables and explained in some detail how the different algebraic elements in the formalism are prepared in an angular momentum decomposition for the numerical implementation.

The bulk of the review has been devoted to a comparison of theory and experiment and to theoretical predictions. The latter ones, if confronted with the data in the future, would challenge the dynamical assumptions even more stringently and systematically than what has been achieved up to now. Our theoretical results which are compared to data are based on the AV18/UrbanaIX Hamiltonian model and one- and the dominant two-body currents related to AV18. The rich set of data comprises elastic electron scattering on ${ }^{3} \mathrm{He}$ and ${ }^{3} \mathrm{H}$, inclusive electron scattering on ${ }^{3} \mathrm{He}$ and ${ }^{3} \mathrm{H}$, nucleon-deuteron radiative capture and the time reversed process of pd photodisintegration of ${ }^{3} \mathrm{He}$ and finally the $3 \mathrm{~N}$ photodisintegration of ${ }^{3}$ He. We tried to include as many as possible of the data situated in our nonrelativistic regime, which we qualitatively defined by $|\vec{Q}| \leq 500 \mathrm{MeV} / \mathrm{c}$ for the virtual photon and the three-nucleon c.m. energy below the pion threshold. Clearly also in that kinematical domain some effects of relativity will be visible but they are not dealt with in this review except for a small study for the elastic electron scattering process on ${ }^{3} \mathrm{He}$.

The elastic form factors of ${ }^{3} \mathrm{He}$ and ${ }^{3} \mathrm{H}$ are rather well described in the low momentum

region $q \leq 3 \mathrm{fm}^{-1}$. The presence of the $3 \mathrm{NF}$ is noticeable and its effect goes in the right direction toward the data. Our results are very similar to the ones achieved by the Hanover group, which rely on a single $\Delta$-isobar admixture model instead of an explicit $3 \mathrm{NF}$. They are also similar to predictions of the Pisa group and collaborators, who apply the same model Hamiltonian as used in this review, but include additional currents. These currents 
when applied in the higher $|\vec{Q}|$-domain not studied in this review significantly improve the agreement with the data.

The two inclusive response functions, $R_{L}$ and $R_{T}$, in inclusive unpolarized electron scattering on ${ }^{3} \mathrm{He}$ and ${ }^{3} \mathrm{H}$ show overall a good agreement between theory and experiment with a slight underestimation, however, of $R_{L}$ in case of ${ }^{3} \mathrm{H}$. Interesting is the interplay of 3NF's and the two-body currents for $R_{T}$, which have a tendency to cancel each other under our (restricted) dynamical assumptions. When a comparison was possible the results by the other groups are very similar to ours. The Pisa and Trento groups are able to include the pp Coulomb force which is an important step forward. It will be very interesting to see its quantitative effect in detailed future studies, especially in the low momentum regime.

If one allows for polarization of the incoming electron and the ${ }^{3} \mathrm{He}$ target two more response functions, $R_{T^{\prime}}$ and $R_{T L^{\prime}}$, in inclusive electron scattering are accessible with related asymmetries. We showed that the sensitivity to the magnetic form factor of the neutron survives in the transversal asymmetry $A_{T^{\prime}}$ despite the fact that all dynamical ingredients, FSI, 3NF effects and MEC's play an important role in the low momentum region. This has been used to extract $G_{M}^{n}$ for $Q^{2}=0.1$ and $0.2(\mathrm{GeV} / \mathrm{c})^{2}$, which are in good agreement with the $G_{M}^{n}$-values extracted using a deuteron target.

We also draw attention to the Coulomb sum rule which in principle is an excellent source of information on two-body correlations modified by two-body density and relativistic effects. Unfortunately, due to strong cancellations the part of the Coulomb sum which carries that information has large error bars, thus an improved set of data would be very informative.

In case of the pd electrodisintegration of ${ }^{3} \mathrm{He}$ we faced both, agreement and disagreement, around the quasi elastic proton knockout peak. This is a quite unsatisfactory situation, especially since a renewed theoretical analysis by the Hanover group with a $\Delta$-isobar admixture and therefore with a different dynamics found very similar results. This deserves further theoretical studies. In case of the deuteron knock out peak we also face disagreement, namely a severe overestimation of the data in the neighborhood of missing momentum $p_{m}=0$ despite the fact, that the $3 \mathrm{NF}$ for the measured configurations moves theory significantly in the direction of the data. For another set of data in parallel deuteron knockout kinematics the agreement with the data looks better but does not include the situation with $p_{m}=0$. In relation to both peak areas we think that the pd electrodisintegration of ${ }^{3}$ He requires further efforts both in experiment and theory, as will be also addressed below. 
In radiative $\mathrm{Nd}$ capture the cross section data are rather well described over a wide range of energies. This is not the case for the spin observables $A_{y}(p), i T_{11}$ and the tensor analyzing powers $T_{i j}$. There remains much room for improvements in the dynamical inputs. An important step forward in that direction has been done very recently by the Pisa group with collaborators. They completed the current related to the AV18/UrbanaIX model Hamiltonian what indeed improved the agreement between the theory and experiment in the very low energy regime. But also there some discrepancies remained in the two vector analyzing powers. Since similar discrepancies are also present in pure Nd scattering [18] they might have a common origin, presumably missing spin structures in the 3NF.

The experimental situation in pd photodisintegration of ${ }^{3} \mathrm{He}$ is quite controversial as has been displayed for the energy dependence of the cross section at a fixed angle and for the integrated cross section.

The photon induced $3 \mathrm{~N}$ breakup of ${ }^{3} \mathrm{He}$ is still a rather unsettled issue. The total breakup cross section data are severely controversial which precludes a conclusion about the validity of the theory for that process. The very few more exclusive data for that complete breakup unfortunately could not be analyzed properly by us since the experimental conditions about angular and energy acceptances were not sufficiently well documented in the literature. In any case, our point geometry results are at least in the neighborhood of those data given in the form $d^{4} \sigma / d \Omega_{1} d \Omega_{2}$.

In view of the existing data we think that more systematic measurements with possibly improved accuracy are needed to get better insight into the validity of the dynamical assumptions. For that aim we provided a few theoretical predictions, some of which at least will hopefully be addressed in future experiments.

The two response functions $\tilde{R}_{T^{\prime}}$ and $\tilde{R}_{T L^{\prime}}$ appearing with the helicity of the incoming electron show a great sensitivity to the dynamical input and especially $\tilde{R}_{T L^{\prime}}$ for ${ }^{3} \mathrm{He}$ shows a strong variation in shape as a function of $|\vec{Q}|$.

The electron induced pd breakup of ${ }^{3} \mathrm{He}$ poses questions. For the proton knockout peak region we have shown three quite different cases. One is affected separately by FSI and by the 3NF, another one is predominantly just given by PWIA alone, and a third one just by FSI with no effect of the $3 \mathrm{NF}$. The second one would be especially important to be verified by experiment, since only the simplest ingredients enter, the ${ }^{3} \mathrm{He}$ state, the deuteron state, and the single nucleon current. In all three cases the effects of MEC's are negligible. 
In case of the deuteron knockout peak we also have selected three different situations in relation to the strength of the FSI effect and the MEC contributions. Since the deuteron is composite the mechanism of knockout is more complex than for the nucleon knockout.

Despite the fact that the concept of the spectral function has been widely used in the literature we think that a more systematic approach to the situations where it is predicted to be useful and where it fails would be adequate. We displayed two examples out of many described before in [190].

The semiexclusive process $\overrightarrow{3} \mathrm{He}\left(\vec{e}, e^{\prime} N\right) N N$, where both initial particles are polarized, would be also an interesting source of information about the interplay of dynamical ingredients. We showed two kinematical conditions. In one the asymmetry $A_{\|}$for the upper end of the knocked out neutron energy spectrum would be suitable to extract $G_{M}^{n}$, since all curves, PWIA, FSI23, FSI, FSI + MEC and finally FSI+MEC+3NF coincide there. In the other case the PWIA result differs strongly from the others and large corrections are necessary. The asymmetry $A_{\perp}$, which in PWIA is proportional to $G_{E}^{n} \cdot G_{M}^{n}$, requires in both chosen kinematical configurations always strong corrections from FSI. Since ${ }^{3} \mathrm{He}$ carries little proton polarization the corresponding asymmetries are strongly influenced by final state interactions. For one kinematical condition we found that FSI23 alone would be quite misleading for $A_{\|}$but completely sufficient for $A_{\perp}$, while for the other kinematical condition 3NF effects are significant for both asymmetries except at the upper end of the proton energy spectrum, where all curves (except PWIA) coincide. We think that also these different scenarios deserve a systematic experimental study.

We also investigated the question, whether the two-nucleon relative momentum distribution inside ${ }^{3} \mathrm{He}$ could be approached experimentally. We showed that in our kinematical regime this is not possible but at least for proton knockout under parallel kinematics the FSI23 dynamics is sufficient. Thus the relative momentum distribution folded with the NN t-matrix would be accessible, except for an additional small shift caused by the action of the 3NF. In the case of the neutron knockout only the transversal response function exhibits that feature. For high $|\vec{Q}|$-values, however, also $R_{L}$ can be expected to behave similarly.

Finally, in the field of electrodisintegration we investigated the spin dependent momentum distribution for polarized proton-deuteron clusters in polarized ${ }^{3} \mathrm{He}$. For the processes $\overrightarrow{{ }^{3} \mathrm{He}}\left(e, e^{\prime} \vec{p}\right) d$ and $\overrightarrow{{ }^{3} \mathrm{He}}\left(e, e^{\prime} \vec{d}\right) p$ under fully collinear condition it turned out, that only for rather low $p_{d}$ momenta we found a tendency that the two responses $R_{L}$ and $R_{T}$ properly 
divided by the electromagnetic nucleon form factors approach the sought-for momentum distributions for increasing $|\vec{Q}|$-values; otherwise FSI and 3NF effects preclude that. Nevertheless a measurement of that polarized setup would be quite interesting since all the dynamics comes into the play.

$3 \mathrm{~N}$ photodisintegration of ${ }^{3} \mathrm{He}$ comprises a lot of detailed dynamical information. We found that the semiexclusive reactions ${ }^{3} H e(\gamma, p) p n$ and ${ }^{3} H e(\gamma, n) p p$ show quite a different dependence on the emitted nucleon energy and the emission angles. In all cases the $3 \mathrm{NF}$ effects are mostly washed out due to the integration over part of the phase space. To the best of our knowledge no data are available, but they would be very informative.

If one allows for polarization for the incoming photon and $/$ or ${ }^{3} \mathrm{He}$, analyzing powers and spin correlation coefficients can be measured in the semiexclusive processes. We found that at $E_{\gamma}=12 \mathrm{MeV}$, especially for neutron emission, 3N force effects are quite significant in $A_{y}^{3} \mathrm{He}$ and in the spin correlation coefficients, while $A_{x}^{\gamma}$ has no noticeable $3 \mathrm{NF}$ dependence. At $E_{\gamma}=40 \mathrm{MeV}$ the $3 \mathrm{NF}$ effects have essentially disappeared. No data are available to the best of our knowledge.

Our last predictions in this review are for the most informative process, the exclusive ${ }^{3} \mathrm{He}(\gamma, p p) n$ reaction. We scanned the full phase space for $3 \mathrm{NF}$ effects and located the regions where they are as large as $20 \%$ and above. Even after averaging over certain angular and energy intervals carried out in two examples, the magnitudes of these effects survived. Precise and well documented data (for future analysis and possibly new dynamics) would be very important.

The comparison of data and theory in this review clearly demonstrated that the chosen dynamics, forces and currents, is more or less adequate. In most cases we encountered fair to good agreement with the data but also in some cases clear discrepancies. Since for pure hadronic processes in few-nucleon systems, especially in the well investigated $3 \mathrm{~N}$ continuum, the AV18/UrbanaIX Hamiltonian model leads to similar agreements and disagreements, the reason for certain discrepancies in the electromagnetically induced processes cannot be searched alone in the additional ingredient, the electromagnetic current operator, but also in the deficiencies of that Hamiltonian model. Certainly additional spin structures in the $3 \mathrm{NF}$ model are required. This has been already noticed in pure 3N scattering [18, 234-237] but also in the description of spectra of light nuclei [26, 238]. Additional 3NF models introduced recently [239] improved the theoretical spectra. Therefore, proceeding in this manner and 
allowing for corresponding additional currents and relativistic features might be one way to go to achieve more quantitative results.

Another approach emerged in recent years based on effective field theory, either in the pion-less form or explicitly including the pion degrees of freedom in a form constrained by spontaneously broken chiral symmetry and including explicitly broken parts. This is a systematic approach which is controlled in the low momentum region by a smallness parameter. Therefore the predictions can be improved systematically and theoretical errors can be estimated. This new approach to low energy nuclear physics is very promising. It relies on effective Langragians, which allow for well defined couplings to electroweak fields, provides internal connections between NN and many-nucleon forces, and generates systematically relativistic corrections. Of course this approach is restricted to generic external momenta below a certain mass scale.

We refer the reader to several reviews [240] on these kind of approaches and cite only a short subjective list of papers out of very many, which we think are very relevant to investigate few-nucleon systems without and with electroweak probes. More references can be found there. The approach to nuclear forces based on effective field theory constrained by chiral symmetry goes back to S. Weinberg [241]. First applications were pioneered in [242]. This was followed up in an extended and improved manner in [243-245] pushing NN forces to next-to-next-to-next-to leading order $\left(\mathrm{N}^{3} \mathrm{LO}\right)$ in the chiral expansion. Thereby it has to be emphasized that the 3NF's and beyond are consistent to the NN forces. Various applications [76, 246] clearly demonstrated the success of that approach. In the pion-less form, restricted to a lower momentum regime, also convincing successful strides have been performed [247]. Coupling to electroweak fields has been investigated without and with explicit pions [248-250]. We expect that these approaches will put low energy nuclear physics including electroweak processes on a firm ground and will enable well founded applications like for astrophysical issues.

This review has been closed in January 2005 . We would like to apologize to the authors whose work has not been sufficiently well presented or whose work has not been cited at all. 


\section{Acknowledgments}

This work was supported by the Polish Committee for Scientific Research under grant no. 2P03B00825, by the NATO grant no. PST.CLG.978943, and by DOE under grants nos. DE-FG03-00ER41132 and DE-FC02-01ER41187. One of us (W.G.) would like to thank the

Foundation for Polish Science for the financial support during his stay in Kraków. The numerical calculations have been performed on the Cray SV1 and T3E and on the IBM Regatta p690+ of the NIC in Jülich, Germany.

[1] E. Wigner, Phys. Rev. 43 (1933) 252.

[2] E. Gerjuoy, J. Schwinger Phys. Rev. 61 (1942) 138.

[3] H. Collard et al., Phys. Rev. Lett. 11 (1963) 132.

[4] L.I. Schiff, H. Collard, R. Hofstadter, A. Johansson, M.R. Yearian, Phys. Rev. Lett. 11 (1963) 387.

[5] A. Laverne, C. Gignoux, Nucl. Phys. A203 (1973) 597; S. P. Merkuriev, C. Gignoux, A. Laverne, Ann Phys. 99 (1976) 30.

[6] R.A. Brandenburg, Y.E. Kim, A. Tubis, Phys. Rev. C12 (1975) 1368.

[7] Ch. Hajduk, P.U. Sauer, Nucl. Phys. A322 (1979) 329.

[8] G.L. Payne, J.L. Friar B.F. Gibson, I.R. Afnan, Phys. Rev. C22 (1980) 823.

[9] T. Sasakawa, H. Okuno, T. Sawada, Phys. Rev. C23 (1981) 905.

[10] W. Glöckle, Nucl. Phys. A381 (1982) 343.

[11] C.R. Chen, G.L. Payne, J.L. Friar, B.F. Gibson, Phys. Rev. C31 (1985) 2266; Phys. Rev. C33 (1986) 1740.

[12] T. Sasakawa, S. Ishikawa, Few-Body Systems 1 (1986) 3; S. Ishikawa, T. Sasakawa, Few-Body Systems 1 (1986) 134.

[13] A. Stadler, W. Glöckle, P.U. Sauer, Phys. Rev. C44 (1991) 2319.

[14] Y. Wu, S. Ishikawa, T. Sasakawa, Few-Body Systems 15 (1993) 145.

[15] A. Nogga, A. Kievsky, H. Kamada, W. Glöckle, L.E. Marcucci, S. Rosati, M. Viviani, Phys. Rev. C67 (2003) 034004 and references therein.

[16] H. Witała, T. Cornelius, W. Glöckle, Few-Body Systems 3 (1988) 123. 
[17] J. L. Friar et al., Phys. Rev. C42 (1990) 1838.

[18] W. Glöckle, H. Witała, D. Hüber, H. Kamada, J. Golak, Phys. Rep. 274 (1996) 107.

[19] A. Kievsky, M. Viviani, S. Rosati, Phys. Rev. C64 (2001) 024002; M.Viviani A.Kievski S.Rosati, Few-Body Systems 30 (2001) 39.

[20] W. Glöckle, in "Scattering", editors R.Pike, P.Sabatier, Academic Press 2002 p 1339.

[21] S. Nemoto, K. Chmielewski, S. Oryu, P.U. Sauer, Phys. Rev. C58 (1998) 2599.

[22] J. Goldemberg and R. H. Pratt, Rev. Mod. Phys. 38, (1966) 311.

[23] Y.E. Kim, A. Tubis, Ann. Rev. Nucl. Sci. 24 (1974) 69.

[24] C. Ciofi degli Atti, Prog. Part. Nucl. Phys. 3, (1980) 163.

[25] J. F. Mathiot, Phys. Rep. 173 (1989) 63.

[26] J. Carlson, R. Schiavilla, Rev. Mod. Phys. 70 (1998) 743 and references therein.

[27] S. Boffi, C. Giusti, F.D. Pacati, M.Radici, Electromagnetic Response of Atomic Nuclei, Clarendon Press, Oxford, 1996.

[28] D.J. Klepacki, Y.E. Kim, R.A. Brandenburg, Nucl. Phys. A550 (1992) 53.

[29] L.D. Faddeev, Zh. Eksp. Theor. Fiz. 39 (1960) 1459; Sov. Phys. JETP 12 (1961) 1041.

[30] E.O. Alt, P. Grassberger, W. Sandhas, Nucl. Phys. B2 (1967) 167.

[31] D.R. Lehman, Phys. Rev. Lett. 23 (1969) 1339; Phys. Rev C3 (1971) 1827.

[32] I.R. Barbour, A.C. Phillips, Phys. Rev. Lett. 19 (1967) 1388; Phys. Rev. C1 (1970) 165.

[33] B. F. Gibson and D. R. Lehman, Phys. Rev. C11 (1975) 29; C13 (1976) 477.

[34] D. R. Lehman, F. Prats, and B. F. Gibson, Phys. Rev C19 (1979) 310.

[35] C. R. Heimbach, D. R. Lehman, J. S. O'Connell, Phys. Lett. 66B, 1 (1977). Phys. Rev C16, 2135 (1977).

[36] E. van Meijgaard and J.A.Tjon, Phys.Rev C42 (1990) 74; C42 (1990) 96; C45 (1992) 1463.

[37] J. Jourdan, M. Baumgartner, S. Burzynski, P. Egelhof, R. Henneck, A. Klein, M.A. Pickar, G.R. Plattner, W.D. Ramsay, H.W. Roser, I. Sick, J. Torre, Nucl. Phys. A453 (1986) 220.

[38] R. V. Reid, Ann. Phys. 50 (1968) 411.

[39] S. Ishikawa, T. Sasakawa, Phys. Rev. C45 (1992) R1428.

[40] A.C. Fonseca, D.R. Lehman, Phys. Lett B267 (1991) 159; Few-Body Systems Suppl. 6 (1992) 279; Phys. Rev. C48 (1993) R503.

[41] G. J. Schmid, R. M. Chasteler, H. R. Weller, D. R. Tilley, A. C. Fonseca, D. R. Lehman, Phys. Rev C53 (1996) 35. 
[42] A. C. Fonseca, D. R. Lehman, Few-Body Systems 28 (2000) 189.

[43] J.L. Friar, B.F. Gibson, G.L. Payne, Phys. Lett B251 (1990) 11.

[44] R. Schiavilla, Phys. Lett B218 (1985) 1; R. Schiavilla, V.G. Pandharipande, Phys. Rev. C36 (1987) 2221.

[45] J. Carlson, R. Schiavilla, Phys.Rev. Lett. 68 (1992) 3682; Phys. Rev. C49 (1994) R2880.

[46] V.D. Efros, W. Leidemann, G. Orlandini, Few-Body Systems 14 (1993) 15.

[47] V.D. Efros, W. Leidemann, G. Orlandini, Phys. Lett. B338 (1994) 130.

[48] S. Ishikawa, H. Kamada, W. Glöckle, J. Golak, H. Witała, Nuovo Cimento A107 (1994) 305.

[49] J. Golak, H. Kamada, H. Witała, W. Glöckle, S. Ishikawa, Phys. Rev. C51 (1995) 1638.

[50] S. Ishikawa, H. Kamada, W.Glöckle, J. Golak, H. Witała, Phys. Lett. B339 (1994) 293.

[51] J. Golak, H. Witała, H. Kamada, S. Ishikawa, W. Glöckle, Phys. Rev C52 (1995) 1216.

[52] D.O. Riska, Phys. Scr. 31 (1985) 107.

[53] D.O. Riska, Phys. Scr. 31 (1985) 471.

[54] R.B. Wiringa, V.G.J. Stoks, R. Schiavilla, Phys. Rev. C51 (1995) 38.

[55] A. Buchmann, W. Leidemann, H. Arenhövel, Nucl. Phys. A443 (1985) 726.

[56] K. Ohta, Nucl. Phys. A495 (1989) 564; Phys. Rev. C39 (1989) 2302.

[57] H. Arenhövel, M. Sanzone, "Photodisintegration of the deuteron" in Few-Body Systems Suppl. 3 (1991) 1.

[58] R. Gilman, F. Gross, Journal Phys. G: Nucl Particle Physics 28 (2002) R37.

[59] M. Viviani, A. Kievsky, L.E. Marcucci, S. Rosati, R. Schiavilla, Phys. Rev. C61 (2000) 064001.

[60] J. Carlson, Phys. Rev. C36 (1987) 2026; S.C. Pieper, K. Varga, R.B. Wiringa, Phys. Rev. C66 (2002) 044310.

[61] V.D. Efros, W. Leideman, G. Orlandini, Few-Body Systems 26 (1999) 251.

[62] N. Barnea, W. Leideman, G. Orlandini, Phys. Rev. C61 (2000) 054001.

[63] G. Orlandini, Nucl. Phys. A737 (2004) 210.

[64] W. Leidemann, S. Bacca, N. Barnea, G. Orlandini, Nucl. Phys. A737 (2004) 231.

[65] L.P. Yuan, K. Chmielewski, M. Oelsner, P. U. Sauer, J. Adam Jr., Phys. Rev. C66 (2002) 054004 .

[66] L. P.Yuan, K. Chmielewski, M. Oelsner, P. U. Sauer, A. C. Fonseca, J. Adam Jr., Few-Body Systems 32 (2002) 83. 
[67] A. Deltuva, L.P. Yuan, J. Adam Jr, P.U. Sauer, Phys. Rev. C70 (2004) 034004.

[68] J.M. Laget, Phys. Lett B 609 (2005) 49.

[69] W. Glöckle, The Quantum Mechanical Few-Body Problem, Springer-Verlag, Berlin Heidelberg New York Tokyo 1983.

[70] C. Möller, K. Dan. Vidensk. Selsk. Mat. Fys. Medd. 23 (1943) 1; H. Ekstein, Phys. Rev. 101 (1956) 880 ; J.M. Jauch, Helv. Phys. Acta 31 (1958) 127; 31 (1958) 661; J. Hunziker, in Lecture Notes in Physics, ed. by A.O. Barut, W.E. Brittin (Gordon and Breach, New York 1968) p.1.

[71] D. Hüber, H. Kamada, H. Witała, W. Glöckle, Acta Phys. Pol. B28 (1997) 1677.

[72] A. Bömelburg, W. Glöckle, W. Meier, in Few-Body Problems in Physics, edited by B. Zeitnitz (Elsevier, Amsterdam, 1984), Vol. II, p. 483.

[73] S. Ishikawa, J. Golak, H. Witała, H. Kamada, W. Glöckle, D. Hüber, Phys. Rev. C57 (1998) 39.

[74] S.C. Pieper, R.B. Wiringa, Ann. Rev. Nucl. Part. Sci. 51 (2001) 53.

[75] U. van Kolck, Phys. Rev. C49 (1994) 2932.

[76] E. Epelbaum, A. Nogga, W. Glöckle, H. Kamada, Ulf-G. Meißner, and H. Witała, Phys. Rev. C66 (2002) 064001.

[77] F. Villars, Helv. Phys. Acta. 20 (1947) 476.

[78] H. Miyazawa, Prog. Theor. Phys. 6 (1951) 801.

[79] D.O. Riska, Phys. Rep. 181 (1989) 207.

[80] A.J.F. Siegert, Phys. Rev. 52 (1937) 787.

[81] T. de Forest, J.D. Walecka, Adv. Phys. 15 (1966) 1.

[82] J.L. Friar, Annals of Phys. 81 (1973) 332.

[83] H.-W. Hammer, Ulf-G. Meißner, Eur. Phys. J. A20 (2004) 469; P. Mergell, Ulf-G. Meißner, D. Drechsel, Nucl. Phys. A596 (1996) 367.

[84] J. Jourdan, Nucl. Phys. A721 (2003) 395c; H. Schmieden, Nucl. Phys. A737 (2004) 147; Kees de Jager, Eur. Phys. J. A191 (2004) s01,267.

[85] A. R. Edmonds, Angular Momentum in Quantum Mechanics, Princeton University Press 1960.

[86] M.E. Rose Elementary Theory of Angular Momentum, Dover Publication 1995.

[87] M. Chemtob, M. Rho, Nucl. Phys. A163 (1971) 1; A212, 628 (1973). 
[88] R. Schiavilla, V.R. Pandharipande, D.O. Riska, Phys. Rev. C40 (1989) 2294.

[89] D.O. Riska, Progress in Particle and Nuclear Physics 11 (1984) 199.

[90] J. Carlson, D.O. Riska, R. Schiavilla, R.B. Wiringa, Phys. Rev. C42 (1990) 830.

[91] R. Schiavilla, V.R. Pandharipande, D.O. Riska, Phys. Rev C40 (1989) 2294.

[92] L.E. Marcucci, M. Viviani, R. Schiavilla, A. Kievsky, S. Rosati, nucl-th/0411082v1; nuclth/0411083v1.

[93] L. E. Marcucci, M. Viviani, R. Schiavilla, A. Kievsky, S. Rosati, nucl-th/0502048.

[94] G. Sachs, Phys. Rev. 74 (1948) 433.

[95] E.M. Nyman, Nucl. Phys. B1 (1967) 535.

[96] J. A. Wheeler, Phys. Rev. 50 (1936) 643.

[97] G.E. Brown, Mannque Rho, Phys. Rep. 396 (2004) 1.

[98] M. Harada, K. Yamawaki, Phys. Rep. 381 (2003) 1.

[99] T.W. Donnelly, A.S. Raskin, Ann. Phys. (N.Y.) 169 (1986) 247.

[100] H. Collard, R. Hofstadter, E.B. Hughes, A. Johansson, M.R. Yearian, R.B. Day, R.T. Wagner, Phys Rev 138 (1965) B57.

[101] H. Kamada, W. Glöckle, J. Golak, Nuovo Cimento 105A (1992) 1435.

[102] T.de Forest Jr, Nucl. Phys. A392 (1983) 232.

[103] G.G. Ohlsen, Nucl. Instr. Meth. 37 (1965) 240.

[104] V. N. Litvinenko et al., Phys. Rev. Lett. 78 (1997) 4569; W. Tornow et al., Phys. Lett. B574 (2003) 8 .

[105] H. Liu, Ch. Elster, W. Glöckle, nucl-th/0410051.

[106] D. Hüber, H. Kamada, H. Witała, W. Glöckle, Few-Body Systems 16 (1994) 165.

[107] V.V.Kotlyar, H. Kamada, W. Glöckle, J. Golak, Few-Body Systems 28 (2000) 35.

[108] D. Hüber, H. Witała, A. Nogga, W. Glöckle, H. Kamada, Few-Body Systems 22 (1997) 107.

[109] W. Glöckle, G. Hasberg, A.R. Neghabian Z.Phys. A305 (1982) 217.

[110] H. Witała, W. Glöckle, Th. Cornelius, Phys. Rev. C39 (1989) 384.

[111] H. Witała, W. Glöckle, H.Kamada, Phys. Rev. C43 (1991) 1619.

[112] R. Skibiński, Ph.D. thesis, Jagiellonian University, Kraków, 2002 (www.if.uj.edu.pl/ZFJ/prj.old-010501/fb/thesis2rs.html).

[113] R. Skibiński, J. Golak, H. Witała, W. Glöckle, A. Nogga, Eur. Phys. J. A24 (2005) 31.

[114] B.S. Pudliner, V.R. Pandharipande, J. Carlson, Steven C. Pieper and R. B. Wiringa, Phys. 
Rev. C56 (1997) 1720.

[115] A. H. Wapstra, G. Audi, Nucl. Phys. A432 (1985) 1.

[116] W.M. Kloet, J.A. Tjon, Phys. Lett. B49 (1974) 419.

[117] A. Barroso, E. Hadjimichael, Nucl. Phys. A238 (1975) 422.

[118] J. L. Friar, B. F. Gibson, G. L. Payne, Phys. Rev C35 (1987) 1502.

[119] R. Schiavilla, V.R. Pandharipande, D.O. Riska, Phys. Rev. C41 (1990) 309.

[120] W. Strueve, Ch. Hajduk, P. U. Sauer, W. Theis, Nucl. Phys. A465 (1987) 651.

[121] L. E. Marcucci, D. O. Riska, R. Schiavilla, Phys. Rev. C58, 3069 (1998).

[122] A. Amroun et al., Nucl. Phys. A579 (1994) 596 and references therein; I. Sick and J. Jourdan, private communication.

[123] R.B. Wiringa, Phys. Rev. C43 (1991) 1585.

[124] H. Henning, J. Adam Jr., P. U. Sauer, A. Stadler, Phys. Rev. C52 (1995) R471.

[125] G. Rupp, J. A. Tjon, Phys. Rev. C45 (1992) 2133.

[126] D.R. Tilley, H.R. Weller, H.H. Hasan, Nucl. Phys. A474 (1987) 1.

[127] K. Dow et al., Phys. Rev. Lett. 61 (1988) 1706.

[128] C. Marchand et al., Phys. Lett. B153 (1985) 29.

[129] G.A. Retzlaff et al., Phys. Rev. C49 (1994) 1263; G.A. Retzlaff, private communication.

[130] V.D. Efros, W. Leidemann, G. Orlandini, E.L. Tomusiak, Phys. Rev. C69 (2004) 044001.

[131] R.S. Hicks et al., Phys. Rev. C67 (2003) 064004.

[132] E.C. Jones Jr., W.L. Bendel, L.W. Fagg, R.A. Lindgren, Phys. Rev. C19 (1979) 610.

[133] S. Martinelli, H. Kamada, G. Orlandini, W. Glöckle, Phys. Rev. C52 (1995) 1778.

[134] R. Machleidt, Adv. Nucl. Phys. 19 (1989) 189.

[135] S.A. Coon, H.K. Han, Few-Body Systems 30 (2001) 131.

[136] J. Carlson, R. Schiavilla, Few-Body System, Suppl. 7 (1994) 3459.

[137] J. Carlson, R. Schiavilla, Phys. Rev. Lett. 68 (1992) 3682.

[138] J. Carlson, R. Schiavilla, Phys. Rev. C49 (1994) R2880.

[139] J. Carlson, J. Jourdan, R. Schiavilla, I. Sick, Phys. Rev. C66 (2002) 024002.

[140] B. Blankleider, R.M. Woloshyn, Phys. Rev. C29 (1984) 538.

[141] J. Golak, G. Ziemer, H. Kamada, H. Witała, W. Glöckle, Phys. Rev. C63 (2001) 034006.

[142] W. Xu et al., Phys. Rev. Lett. 85 (2000) 2900.

[143] G. Höhler et al., Nucl. Phys. B114 (1976) 505. 
[144] H. Anklin et al., Phys. Lett. B336 (1994) 313.

[145] H. Anklin et al., Phys. Lett. B428 (1998) 248.

[146] H. Gao et al., Phys. Rev. C50 (1994) R546; H. Gao, Nucl. Phys. A631 (1998) 170c.

[147] W. Xu et al., Phys. Rev. C67 (2003) 012201.

[148] A. Kievski, E. Pace, G. Salme, M. Viviani, Phys. Rev. C56 (1997) 64.

[149] F. Xiong et al., Phys. Rev. Lett. 87 (2001) 242501.

[150] R. Schiavilla, R.B. Wiringa, J. Carlson, Phys. Rev. Lett. 70 (1993) 3856.

[151] J. Golak, H. Witała, H. Kamada, D. Hüber, S. Ishikawa, W. Glöckle, Phys. Rev. C52 (1995) 1216.

[152] P.H.M. Keizer et al., Phys. Lett. B157 (1985) 255; P.H.M. Keizer et al., Phys. Lett. B197 (1987) 29.

[153] P.H.M. Keizer, Ph.D. thesis, Amsterdam 1986.

[154] E. Jans, private communication.

[155] C.M Spaltro et al., Nucl. Phys. A706 (2002) 403.

[156] C.M. Spaltro et al., Phys. Rev. Lett. 81 (1998) 2870.

[157] R. Skibiński, J. Golak, H. Kamada, H. Witała, W. Glöckle, A. Nogga, Phys. Rev. C67 (2003) 054001 .

[158] F. Goeckner, W.K. Pitts, and L.D. Knutson, Phys. Rev. C45 (1992) R2536.

[159] B.D. Belt, C.R. Bingham, M.L. Halbert, A. van der Woude, Phys. Rev. Lett. 24 (1970) 1120.

[160] W.K. Pitts et al., Phys. Rev. C37 (1988) 1.

[161] M.A. Pickar et al., Phys. Rev. C35 (1987) 37.

[162] G. Mitev et al. Phys. Rev. C34, 389 (1986).

[163] M. Viviani, R. Schiavilla, A. Kievsky, Phys. Rev. C54 (1996) 534.

[164] L. Ma et al., Phys. Rev. C55 (1997) 588.

[165] E.A. Wulf et al., Phys. Rev. C61 (1999) 021601R9.

[166] M. Viviani, L.E. Marcucci, A. Kievsky, S. Rosati, R. Schiavilla, Eur. Phys. J. A17 (2003) 483.

[167] K. Sagara et al., in Few Body Problems in Physics, edited by F. Gross, AIP Conf. Proc. No. 334 (AIP, Woodbury, NY, 1995), p.467; H. Akiyoshi, Ph.D. thesis, Kyushu University, 1997.

[168] T.Klechneva and J.Jourdan private communication.

[169] T. Yagita et al., Mod. Phys. Lett. A18 (2003) 322. 
[170] M.C. Vetterli, J.A. Kuehner, A.J. Trudel, C.L. Woods, R. Dymarz, A.A. Pilt, H.R. Weller, Phys. Rev. Lett. 54 (1985) 1129.

[171] H. Anklin et al., Nucl. Phys. A636 (1998) 189.

[172] J.R. Stewart, R. Morrison, J. O’Connell, Phys. Rev. 138 (1965) B372.

[173] G. Ticcioni, S.N. Gardiner, J.L. Matthews, R.O. Owens, Phys. Lett. 46B (1973) 369.

[174] D.I. Sober, Hall Crannell, B.M.K. Nefkens, W.J. Briscoe, D.H. Fitzgerald, R. Goloskie , W.W. Sapp, Jr., Phys. Rev. C28 (1983) 2234.

[175] N.M. O'Fallon, L. Koester Jr., J. Smith, Phys. Rev. C5 (1972) 1926.

[176] V.N. Fetisov, A.N. Gorbunov, A.T. Varfolomeev, Nucl. Phys. A71 (1965) 305.

[177] W. Wölfli, R. Bösch, J. Lang, R. Müller, P. Marimier, Phys.Lett. 22 (1966) 75.

[178] A. van der Wounde, M.L Halbert, C.R. Bingham, Phys. Rev. Lett. 26 (1971) 909.

[179] J.L. Matthews, T. Kruse, M.E. Williams, R.O. Owens, W. Savin, Nucl. Phys. A223 (1974) 221.

[180] D.M. Skopik, H.R. Weller, N.R. Roberson, S.A. Wender, Phys. Rev. C19 (1979) 601.

[181] S.K. Kundu, Y.M. Shin, G.D. Wait, Nucl. Phys. A171 (1971) 384.

[182] B.L. Berman, L.J. Koester, J.H. Smith, Phys. Rev. C133 (1964) B117.

[183] Y. Nagai, private communication.

[184] D.D. Faul, B.L. Berman, P. Meyer, D.L. Olson, Phys. Rev. C24 (1981) 849.

[185] B.L. Berman, S. Fultz, P. Yergin, Phys. Rev. C10 (1974) 2221.

[186] A.J. Sarty et al., Phys. Rev. C47 (1993) 459.

[187] A. Deltuva, L. P. Yuan, J. Adam Jr., A. C. Fonseca, P. U. Sauer, Phys. Rev. C69 (2004) 034004 .

[188] J. Golak et al., Nucl. Phys. A707 (2002) 365.

[189] N.R. Kolb, P.N. Dezendorf, M.K. Brussel, B.B. Ritchie, J.H. Smith, Phys. Rev. C44 (1991) 37.

[190] J. Golak, H. Witała, R. Skibiński, W. Glöckle, A. Nogga, H. Kamada, Phys. Rev. C70 (2004) 034005 .

[191] C. Ciofi degli Atti, L.P. Kaptari, Phys. Rev. C66 (2002) 044004; Nucl. Phys. A699 (2002) 49c; nucl-th/0407024v2.

[192] J.L. Friar, B.F. Gibson, G.L. Payne, A.M. Bernstein, and T.E. Chapp, Phys. Rev. C42 (1990) 2310. 
[193] J. Becker et al., Eur. Phys. J. A6 (1999) 329.

[194] C. Carasco et al., Phys. Lett. 559 (2003) 41.

[195] J. Bermuth et al., Phys. Lett. B564 (2003) 199.

[196] J. M. Laget, Phys. Lett. B273, 367 (1991); Phys. Lett. B276, 398 (1992).

[197] D.L. Groep et al., Phys. Rev. C63 (2000) 014005.

[198] E. Jans, private communication.

[199] L.B. Weinstein, R. Niyazov, Eur. Phys. J. A19 (2004) s01, 175.

[200] W. Glöckle, H. Kamada, J. Golak, A. Nogga, H. Witała, R. Skibiński, J. Kuroś-Żołnierczuk, Acta Phys. Pol. B32 (2001) 3053.

[201] J. Golak, W. Glöckle, H. Kamada, H. Witała, R. Skibiński, A. Nogga, Phys. Rev. C65 (2002) 064004 .

[202] R. Skibiński, J. Golak, H. Witała, W. Glöckle, H. Kamada, A. Nogga, Phys. Rev. C67 (2003) 054002.

[203] J-O. Adler private communication.

[204] F. Coester, W.N. Polyzou, Phys. Rev. D26 (1982) 1348.

[205] F. Gross, A. Stadler, M.T. Pena, Phys.Rev. C69 (2004) 034007.

[206] A. Stadler, nucl-th/0411058.

[207] E.P. Wigner, Ann. Math. 40 (1939) 149.

[208] P.A.M. Dirac, Rev. Mod. Phys, 21 (1949) 392.

[209] B. Bakamjian, L.H. Thomas, Phys. Rev. 92 (1953) 1300.

[210] L. Foldy, Phys. Rev. 122 (1961) 275.

[211] F. Coester, Helv. Phys. Acta, 38 (1965) 7.

[212] S.N. Sokolov, Dokl. Akad. Nauk SSSR 233 ( 1977) 575; Sov. Phys. Dokl. 22 ( 1977) 198.

[213] B.D. Keister and W.N. Polyzou, Adv. Nucl. Phys. 20 ( 1991), eds. J.W. Negele, E.W. Vogt.

[214] W.N. Polyzou, Annals of Physics, N.Y. 193 (1989) 367.

[215] W.N. Polyzou, J. Math. Phys 43 (2002) 6024.

[216] W.N. Polyzou, Phys. Rev. C68 (2003) 015202.

[217] W. Klink, W.N. Polyzou, Phys. Rev. C54 (1996) 1189.

[218] B. Balian, E. Brezin, Nuovo Cim. 69 (1969) 403.

[219] H.C. Jean, G.L. Payne, W.N. Polyzou, Few-Body Systems 16 (1994) 17.

[220] W.N. Polyzou, Phys. Rev. D32 (1985) 2216. 
[221] E. Sengbusch, W.N. Polyzou, Phys. Rev. C70 (2004) 058201.

[222] F. Coester, W.N. Polyzou, nucl-th/0405082.

[223] J. Carbonell, B. Desplanques, V.A. Karmanov, J.F. Mathiot, Phys. Rep. 300 (1998) 215.

[224] J. Carbonell, V.A. Karmanov, Eur. Phys. J. A6 (1999) 9.

[225] D.B. Day, J.S. McCarthy, T.W. Donnelly, I. Sick, Ann. Rev. Nucl. Sci. 40 (1990) 357.

[226] G.B. West, Phys. Rep. 18 (1975) 263.

[227] C. Ciofi degli Atti, E. Pace, G. Salme, Phys. Rev. C39 (1989) 259; Phys. Rev. C43 (1991) 1155; C. Ciofi degli Atti, Nucl. Phys. A543 (1992) 183; C. Ciofi degli Atti, G. West, Phys. Lett. B458 (1999) 447; D. Faralli, C. Ciofi degli Atti, G. West, in Proceedings of the 2nd International Conference on Perspectives in Hadron Physics, eds S.Boffi, C. Ciofi degli Atti, M. Giannini, World Scientific 2000, p.75.

[228] S.A. Gurvitz, A.S. Rinat, Phys. Rev. C35 (1987) 696; and references therein.

[229] D. Hüber, W. Glöckle, A. Bömelburg, Phys.Rev. C42 (1990) 2342.

[230] W.N. Polyzou, W. Glöckle, Phys. Rev. C53 (1996) 3111.

[231] R. Skibiński, J. Golak, H. Witała, W. Glöckle, Phys. Rev. C59 (1999) 2384.

[232] W.J. Cummings et al., Phys. Rev. Lett. 68 (1992) 293.

[233] S.E. Kuhn et al., Phys. Rev. C50 (1994) 1771.

[234] K. Sekiguchi et al. , Phys. Rev. C70 (2004) 014001.

[235] K. Ermisch et al. , Phys. Rev. C68 (2003) 051001(R).

[236] J. Kuroś-Żołnierczuk et al. , Phys. Rev. C66 (2002) 024004.

[237] H. Witała et al. , Phys. Rev. C63 (2001) 024007.

[238] R.B. Wiringa, S.C. Pieper, J. Carlson, V.R. Pandharipande, Phys. Rev. C62 (2000) 014001.

[239] S.C. Pieper, V.R. Pandharipande, R.B. Wiringa, J. Carlson, Phys. Rev. C64 (2001) 014001;

S.C. Pieper et al, Phys. Rev. C66 (2002) 044310.

[240] S.R. Beane, P.F. Bedaque, W.C. Haxton, D.R. Phillips, M.J.Savage, in: M.Shifman (Ed), At the Frontier of Particle Physics, vol 1,World Scientific, Singapore, 2001, pp.133-269; P.F. Bedaque, U.van Kolck, Ann. Rev. Nucl. Part. Sci. 52 (2002) 339; U.van Kolck, Prog. Part. Nucl. Phys. 43 (1999) 337; V. Bernard, N. Kaiser, Ulf-G. Meißner, Int. J. Mod. Phys. E4 (1995) 193.

[241] S. Weinberg, Nucl. Phys. B363 (1991) 3.

[242] C. Ordonez, L. Ray, U.van Kolck, Phys. Rev. C53 (1996) 2086; J.L. Friar, S.A. Coon, Phys. 
Rev. C49 (1994) 1272; N. Kaiser, R. Brockmann, W. Weise, Nucl. Phys. A625 (1997) 758.

[243] N. Kaiser, Phys. Rev. C61 (1999) 014003; Phys. Rev. C62 (2000) 024001; Phys. Rev. C64 (2001) 057001.

[244] E. Epelbaoum, W. Glöckle, Ulf-G. Meißner, Nucl. Phys. A637 (1998) 107; Nucl. Phys. A671 (2000) 295; Eur. Phys. J. A19 (2004) 125; A19 (2004) 401; Nucl. Phys. A747 (2005) 362.

[245] D.R. Entem, R. Machleidt, Phys. Rev. C68 (2003) 041001.

[246] E. Epelbaum, H. Kamada, A. Nogga, H. Witała, W. Glöckle, Ulf-G. Meißner, Phys. Rev. Lett. 21 (2001) 4787; E. Epelbaum, A. Nogga, W. Glöckle, H. Kamada, Ulf-G. Meißner, H.Witała, Eur. Phys. J. A15 (2002) 543; E. Epelbaum, A.Nogga, H. Witała, H. Kamada, W. Glöckle, Ulf-G.Meißner, Eur. Phys. J. A17 (2003) 415; W. Glöckle, E. Epelbaum, H. Kamada, Ulf-G. Meißner, A. Nogga, H.Witała, Eur. Phys. J. A19 (2004) s01,159.

[247] P.F. Bedaque, H-W. Hammer, U.van Kolck, Nucl. Phys. A676 (2000) 357; P.F. Bedaque, G. Rupak, H.W. Griesshammer, H-W. Hammer, Nucl.Phys. A714 (2003) 589; H.W. Griesshammer, nucl-th/0502039v1; Nucl. Phys. A744 (2004) 192.

[248] M. Rho, Phys. Rev. Lett. 66 (1991) 1275.

[249] B. Kubis, Ulf-G. Meißner, Nucl. Phys. A679 (2001) 698; T.-Sun Park, D.-P. Min, Mannque Rho, Nucl. Phys. A596 (1996) 515; S. Beane, M. Malheiro, J.A. McGovern, D.R. Phillips, U.van Kolck, nucl-th/0403088v1; K.Kubodera, nucl-th/0404027v1; M.Walz et al. , Phys. Lett. B513 (2001) 37.

[250] D.B. Kaplan, M.J. Savage, M.B. Wise, Phys. Rev. C59 (1999) 617; J.-Wei Chen, G. Rupak, M.J. Savage, Nucl. Phys. A653 (1999) 386; G. Rupak, Nucl. Phys. A678 (2000)405; H. Sadeghi, S. Bayegan, nucl-th/0411114. 


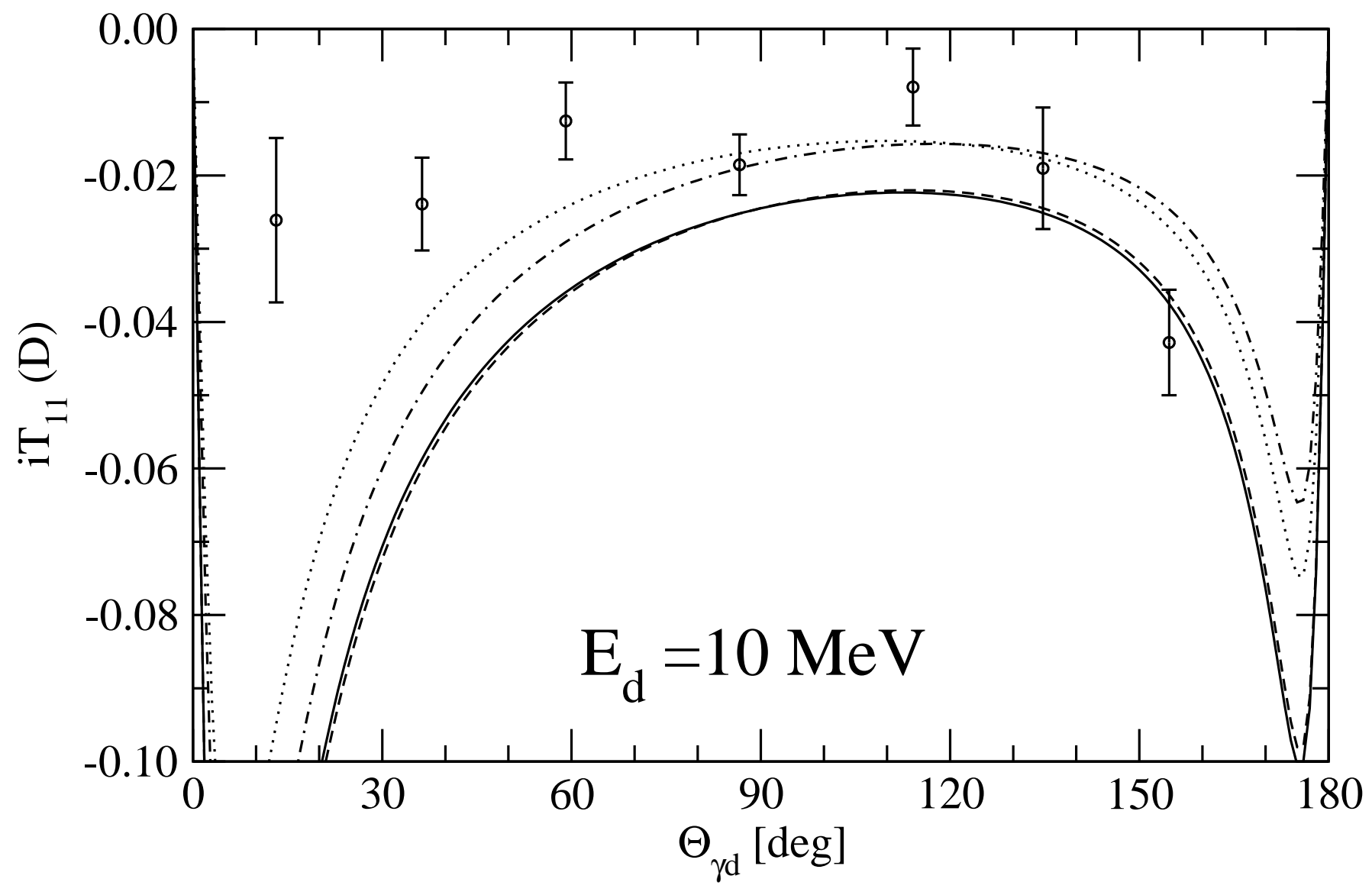


This figure "fig54.jpg" is available in "jpg" format from: http://arxiv.org/ps/nucl-th/0505072v1 
This figure "fig57.jpg" is available in "jpg" format from: http://arxiv.org/ps/nucl-th/0505072v1 
This figure "fig58.jpg" is available in "jpg" format from: http://arxiv.org/ps/nucl-th/0505072v1 
This figure "fig59.jpg" is available in "jpg" format from: http://arxiv.org/ps/nucl-th/0505072v1 
This figure "fig76.jpg" is available in "jpg" format from: http://arxiv.org/ps/nucl-th/0505072v1 
This figure "fig77.jpg" is available in "jpg" format from: http://arxiv.org/ps/nucl-th/0505072v1 
This figure "fig82.jpg" is available in "jpg" format from: http://arxiv.org/ps/nucl-th/0505072v1 
This figure "fig83.jpg" is available in "jpg" format from: http://arxiv.org/ps/nucl-th/0505072v1 
This figure "fig84.jpg" is available in "jpg" format from: http://arxiv.org/ps/nucl-th/0505072v1 
This figure "fig85.jpg" is available in "jpg" format from: http://arxiv.org/ps/nucl-th/0505072v1 
This figure "fig92.a.jpg" is available in "jpg" format from: http://arxiv.org/ps/nucl-th/0505072v1 
This figure "fig92.b.jpg" is available in "jpg" format from: http://arxiv.org/ps/nucl-th/0505072v1 
This figure "fig93.a.jpg" is available in "jpg" format from: http://arxiv.org/ps/nucl-th/0505072v1 
This figure "fig93.b.jpg" is available in "jpg" format from: http://arxiv.org/ps/nucl-th/0505072v1 
This figure "fig94.a.jpg" is available in "jpg" format from: http://arxiv.org/ps/nucl-th/0505072v1 
This figure "fig94.b.jpg" is available in "jpg" format from: http://arxiv.org/ps/nucl-th/0505072v1 
This figure "fig95.jpg" is available in "jpg" format from: http://arxiv.org/ps/nucl-th/0505072v1 
This figure "fig96.jpg" is available in "jpg" format from: http://arxiv.org/ps/nucl-th/0505072v1 
This figure "fig97.jpg" is available in "jpg" format from: http://arxiv.org/ps/nucl-th/0505072v1 
This figure "fig98.jpg" is available in "jpg" format from: http://arxiv.org/ps/nucl-th/0505072v1 
This figure "fig99.jpg" is available in "jpg" format from: http://arxiv.org/ps/nucl-th/0505072v1 